Universidade de São Paulo

Instituto de Física

\title{
Emaranhamento multicor para redes de informação quântica
}

\author{
Antonio Sales Oliveira Coelho
}

Tese de doutorado apresentada ao

Instituto de Física para a obtenção do título de Doutor em Ciências

Orientador: Prof. Dr. Paulo Alberto Nussenzveig

Banca Examinadora:

Prof. Dr. Daniel Felinto Pires Barbosa (UFPE)

Prof. Dr. Marcelo Paleologo Elefteriadis de França Santos (UFMG)

Prof. Dr. Gustavo Garcia Rigolin (UFSCAR)

Prof. Dr. Alexandre Levine (IFUSP) 
FICHA CATALOGRÁFICA

Preparada pelo Serviço de Biblioteca e Informação do Instituto de Física da Universidade de São Paulo

Coelho, Antonio Sales Oliveira

Emaranhamento multicor para redes de informação quântica. -São Paulo, 2013.

Tese (Doutorado) - Universidade de São Paulo.

Instituto de Física. Departamento de Física Experimental.

Orientador: Prof. Dr. Paulo Alberto Nussenzveig

Área de Concentração: Física

Unitermos: 1. Informação quântica; 2. Feixes ópticos;

3. Física Experimental.

USP/IF/SBI-034/2013 


\section{Resumo}

Apresentamos nesta tese a primeira geração direta de emaranhamento tripartite entre feixes intensos de luz. Medimos o emaranhamento entre os feixes sinal, complementar e bombeio refletido, produzidos por um Oscilador Paramétrico Ótico (OPO) operando acima do limiar de oscilação. A principal característica do nosso sistema é a diferença entre os comprimentos de onda dos campos $\left(\lambda_{0}=532.251 \mathrm{~nm}, \lambda_{1}=1062.102 \mathrm{~nm}\right.$, e $\left.\lambda_{2}=1066.915 \mathrm{~nm}\right)$. Esta é a primeira medida de emaranhamento entre mais de dois subsistemas produzidos por uma única fonte no âmbito de variáveis contínuas. Para investigar a existência emaranhamento, utilizamos o critério de positividade sob transposição parcial (PPT). Este critério, aplicado a sistemas compostos por estados gaussianos, dispostos em bipartições do tipo $1 \times(N-1)$, torna-se necessário e suficiente na demonstração do emaranhamento. Realizamos também a caracterização do emaranhamento produzido quando submetemos os campos a perdas, identificando regiões de parâmetros onde os estados apresentam emaranhamento frágil ou robusto. A fim de garantir que estamos lidando com estados gaussianos, investigamos a estatística das flutuações dos campos, mostrando excelente acordo entre os valores dos momentos de ordem mais alta da fotocorrente medida e os valores esperados para uma distribuição gaussiana. O estudo que apresentamos é um passo importante para a elaboração de uma rede de informação quântica que possibilitará a comunicação entre diferentes sistemas físicos. 



\section{Abstract}

We present in this thesis the first direct generation of tripartite entanglement among bright beams of light. We measured the entanglement among signal, idler and reflected pump beams, produced by an Optical Parametric Oscillator (OPO) operating above the threshold. The main feature of our system is the difference between the wavelengths of the fields $\left(\lambda_{0}=532.251 \mathrm{~nm}, \lambda_{1}=1062.102 \mathrm{~nm}\right.$, e $\left.\lambda_{2}=1066.915 \mathrm{~nm}\right)$. This is the first measurement of entanglement among more than two subsystems produced by a single source, in a continuous variable system. In order to investigate the existence of entanglement, we applied the criterion of positivity under partial transposition (PPT). This criterion, applied to systems composed of Gaussian states, arranged in $1 \times(N-1)$ bipartition, becomes necessary and sufficient to demonstrate entanglement. We also characterized the entanglement when the fields are submitted to losses, identifying regions of parameters where the states have fragile or robust entanglement. To ensure that we are dealing with Gaussian states, we investigate the statistics of the fields, showing excelent agreement among values of higher order moments of the photocurrent measured and expected values for a Gaussian distribution. The present study is an important step in the development of a quantum information network formed by different physical systems. 



\section{Publicações}

$\checkmark$ S. N. Filippov, V. I. Man'ko, A. S. Coelho, A. Zavatta and M. Bellini

Single photon-added coherent states: estimation of parameters and fidelity of the optical homodyne detection

Aceito para publicação em Physica Scripta.

$\checkmark$ M. Bellini, A. S. Coelho, S. N. Filippov, V. I. Man'ko, and A. Zavatta.

Towards higher precision and operational use of optical homodyne tomograms

Phys. Rev. A 85, 052129 (2012).

$\checkmark$ F. A. S. Barbosa, A. J. de Faria, A. S. Coelho, K. N. Cassemiro, A. S. Villar, P. Nussenzveig, and M. Martinelli.

Disentanglement in bipartite continuous-variable systems

Phys. Rev. A 84, 052330 (2011).

$\checkmark$ F. A. S. Barbosa, A. S. Coelho, A. J. de Faria, K. N. Cassemiro, A. S. Villar, P. Nussenzveig, and M. Martinelli.

Robustness of bipartite Gaussian entangled beams propagating in lossy channels Nature Photon. 4, 858 (2010).

$\checkmark$ A. S. Coelho, F. A. S. Barbosa, K. N. Cassemiro, A. S. Villar, M. Martinelli and P. Nussenzveig.

Three-Color Entanglement

Science 326, 823 (2009).

$\checkmark$ J. E. S. César, A. S. Coelho, K. N. Cassemiro, A. S. Villar, M. Lassen, P. Nussenzveig, and M. Martinelli.

Extra phase noise from thermal fluctuations in nonlinear optical crystals

Phys. Rev. A 79, 063816 (2009). 



\section{Agradecimentos}

Se eu tivesse que escolher uma palavra para resumir este período de seis anos ${ }^{1}$ que passei na cidade de São Paulo, eu escolheria a palavra amadurecimento. Foi um processo árduo e intenso, mas que trouxe grandes benefícios para todos os aspectos da minha vida e, como tal, eu jamais chegaria até aqui se não fosse a participação de pessoas com as quais eu tive a felicidade de encontrar.

Em primeiríssimo lugar, agradeço à minha família. Meus pais, Maria Vilma e Antonio SALES, meus maiores exemplos de perseverança, paciência e dedicação. Muito obrigado por nunca permitirem que eu desistisse dos meus sonhos. Meus irmãos, Vinícius SAlEs e Dulce SALES, eu os agradeço pelo companheirismo e pela alegria contagiosa com que tratam a vida. Minha esposa, Lilian Rego, presente que meu pai me deu, talvez fossem necessárias mais 140 páginas de tese para exprimir de forma justa meus agradecimentos a você; obrigado pelo companheirismo e ajuda constante, por ponderar minhas dificuldades e pelo exemplo de seriedade profissional. Também te agradeço pelas amizades que ganhei, dentre elas, as imunogenéticas e seus respectivos.

Com enorme entusiasmo, agradeço a meu orientador, Prof. Paulo Nussenzveig. Muito obrigado pelos ensinamentos que transcenderam os muros do mundo acadêmico (é, infelizmente estes muros existem). Algumas de suas características mais marcantes despertam em mim grande admiração, de modo especial, sua intuição científica e seu anseio por um ensino de qualidade. Pelas discussões e paciência meu muito-obrigado. Não menos importante na construção deste trabalho foi o Prof. Marcelo Martinelli. Sou muito grato pela paciência com a qual me ouviu em diversas situações e pela dedicação constante nos ensinamentos sobre os detalhes do experimento.

Faltam-me palavras para agradecer a ajuda incomensurável de duas pessoas pelas quais tenho grande admiração, Alessandro Villar e Katiúscia Cassemiro. A ele agradeço a forte atuação como orientador em minha formação; sou grato também pelos conselhos e cobranças muitas vezes regadas à ironia que lhe é peculiar. A ela agradeço a correção sempre atenta dos meus textos, opiniões e discussões sinceras. Não posso deixar de agradecer a acolhida e atenção que me deram em Erlangen e Recife.

Alguns professores foram fundamentais para minha formação, tanto por meio de uma interação direta quanto pela simples observação de seus atos e posturas dentro do ambiente acadêmico. São eles: Antonio Piza, Renata Funchal, Oscar Éboli, Josif Frenkel e José Pimentel. Agradeço a participação ativa dos membros da banca examinadora desta tese, por aceitarem ler, criticar e avaliar criteriosamente este trabalho.

Agradeço aos colegas de trabalho por tornarem o ambiente no laboratório mais ameno e por terem se segurado para não usar as armas brancas disponíveis quando eu cismava em distribuir apelidos. De modo especial, agradeço a Felippe BARBosa, com quem dividi o tão disputado OPO. Pelas noites em claro realizando medidas e pelas discussões agradáveis, meu

\footnotetext{
${ }^{1}$ Dois anos de mestrado e quatro anos de doutorado.
} 
muito-obrigado. Hans M. Florez (o MAFAlda), um grande amigo; cara, obrigado pelo companheirismo e momentos de descontração regados a samba (graças a Ana Luiza), cerveja e pandeiro. Sou muito grato a Renné Araúso por ter trazido a música para dentro do laboratório, pela simplicidade com que guia as discussões e por ter lido atenciosamente minha tese. Luís Xavier (o Chico Bento), muito obrigado pelas impagáveis soluções dos problemas eletrônicos recorrentes. Agradeço também aos demais colegas: Márcio Miranda, Luciano Cruz, Klara Theófilo, Carlos Gonzalez (o Colombaiano! Que orgulho deste apelido!), Paula Meirelles, Igor Konieczniak (o Flipper), Flávio Moraes (o Ground State) a meiga Rayssa Bruzaca (a Supernanny) e Renato Domeneguetti (o Renacto)... relutei, mas não seria eu se não colocasse os parênteses.

Este trabalho não teria acontecido se não fosse o apoio dos funcionários do Instituto de Física. Às secretárias do departamento, Juliane Pagamice e Edi, obrigado pela eficiência e prestatividade. Ao pessoal da oficina mecânica, em especial o Sr. Wilson Aparecido, José Carlos e Raimundo Moreira, obrigado pelos ensinamentos e agilidade. Agradeço ao pessoal do Centro de Computação, Renata, Antonio, Leonel e Sérgio. Agradeço também a D. GraçA pelo animado "Bom dia" de todos os dias. Agradeço também aos funcionários da CPG, Éber, Cristiano e Izabel por me explicarem pacientemente todos os detalhes da infernal burocracia.

Agradeço às pessoas que me receberam em locais distantes, onde fui buscar elementos essenciais para a minha formação. Pela receptividade na cidade de Florença, agradeço ao amigo e supervisor AlESSANDRo ZAVATta pela forma metódica e dedicada que me apresentou o laboratório e pela confiança depositada. Ao professor MARCO BELLINI pelo bom humor sempre presente e por ter me dado total liberdade para trabalhar; pelo seu exemplo de simplicidade e competência, meu muito-obrigado. Agradeço os momentos descontraídos proporcionados por Constantina Polycarpou e pela companhia no laboratório. Sou grato às pessoas que me ajudaram em diversas questões cotidianas, são elas: Hamit Gurses, LAsti e NALin. Em Recife, contei com a acolhida dos já citados Alessandro Villar e Katiúscia Cassemiro, e com a ajuda crucial dos prestativos Paulo Campos, Nara Cavalcanti, Daniel Felinto, Raquel, Anely Camarotti e André Camaroti, aos quais agradeço imensamente. Agradeço ao Prof. RIOS LEITE por me apresentar o São João recifense.

Durante estes anos, compartilhei com MUITAS pessoas diversas experiências de vida, desde momentos agradáveis até situações deveras complicadas. Pondo-me a citar nomes, inevitavelmente esquecerei alguns, porém, pelos nomes que JAMAIS eu poderia esquecer, ponho-me. Pedro Accioly e Lilian Contin meus queridos "cumpades", obrigado pelas paródias, cervejas, vinhos e reflexões. Juliane Cavalcante e Leonardo Vitoriano, meus primos, por sempre se colocarem presentes e pelas palavras motivadoras, meu muito-obrigado. Agradecer o grande amigo EMídio MARques é tarefa difícil dada a importância que tem, por isso simplesmente digo: muito obrigado. Sou eternamente grato a FÁBIo Jorge, o fisioterapeuta que me devolveu a alegria de viver sem dor. Meus sinceros agradecimentos aos amigos MARINA WALL (a Muretinha) e José Albuquerque (quase um Seu Lunga) que fizeram de São Paulo uma cidade menos dura. Muito obrigado ao querido casal Mário Vieira e Rosana, pelos ensinamentos e carinho. Pelos momentos bem vividos agradeço às pessoas com quem dividi o mesmo 
teto: Milessa Afonso, Jairo Savian, Viviane Angélica, Alan, Wanderson Bento, Paulo Takahashi e Marcelo Alves (o Tatoo). Agradeço os puxões de orelha de Aline Marques e Kitamura. Agradeço também (sem utilizar versalete por uma questão estética) aos demais colegas e amigos que encontrei na USP: Nayara Fonseca, Igor Carboni, Leonardo Lima, Tiago Adorno, Danilo Liarte, Thais, Jorgivan Dias, Thaiany Quevedo, Karine Piacentini, Leandro Bevilaqua, Juliano Neves, Claudio Cruz, Maria Bonacina, Francesca Daprà, Eduardo Do Carmo, Hedhio Luz, Patriota, Jonatas César e os piauienses do CRUSP.

Por fim, agradeço a Fundação de Amparo à Pesquisa do Estado de São Paulo (FAPESP), por ter apostado em mim, financiado meu doutorado e proporcionado conhecer lugares inacreditáveis. 



\section{Sumário}

1 Introdução 1

2 Conceitos fundamentais 5

2.1 Quadraturas do campo: tratamento clássico . . . . . . . . . . . . 6

2.2 Quadraturas do campo: tratamento quântico . . . . . . . . . . . . . . . . 9

2.3 Estados gaussianos e matriz de covariâncias . . . . . . . . . . . . . . 13

2.3.1 Relação entre as médias em $\hat{\rho}$ e $W \ldots$. . . . . . . . . . . . . . 15

2.4 Fotodeteç̧ão de quadraturas do campo . . . . . . . . . . . . . . . . . . . . . . . . . . . . .

2.4.1 O operador de fotodetecção . . . . . . . . . . . . . . . 17

2.4.2 Quadraturas do campo e o processo de fotodetecção . . . . . . . . . 18

2.4.3 Quadraturas segundo análise Fourier . . . . . . . . . . . . . 21

2.4 .4 Espectro de ruído . . . . . . . . . . . . . . . . . . 23

2.4.5 O efeito da mistura de fase . . . . . . . . . . . . . . . . 24

2.5 Referência de ruído . . . . . . . . . . . . . . . . . 26

2.6 O ruído quântico padrão ou shot-noise . . . . . . . . . . . . . . . . 28

2.6.1 Como é medido o shot-noise? . . . . . . . . . . . . . . . . . . 30

2.7 Medidas das flutuações das quadraturas . . . . . . . . . . . . . . . . . . 33

2.7.1 Medidas das quadraturas: detecção homodina . . . . . . . . . . . . 34

2.7 .2 Cavidade ótica vazia . . . . . . . . . . . . . . . . . . 35

2.7.3 Detecção Auto homodina . . . . . . . . . . . . . . . . . . 37

$\begin{array}{lll}3 & \text { Emaranhamento } & 47\end{array}$

3.1 Primeiras reflexões . . . . . . . . . . . . . . . . . . . . . 48

3.2 Estados de Einstein, Podolsky e Rosen . . . . . . . . . . . . . . . . . . 51

3.3 Estados emaranhados segundo Schrödinger . . . . . . . . . . . . . . 52

3.4 O estado de Bohm-Aharonov . . . . . . . . . . . . . . . . . 53

3.5 Definição formal de estados emaranhados . . . . . . . . . . . . . . . 56

3.6 Soma de variâncias: caso bipartite . . . . . . . . . . . . . . . . . . . 60

3.7 Critério de Positividade sob Transposição Parcial (PPT) . . . . . . . . . . 62

3.7 .1 Característica geral . . . . . . . . . . . . . . . 62

3.7 .2 Variáveis discretas . . . . . . . . . . . . . . . . 63 
3.7.3 Variáveis contínuas . . . . . . . . . . . . . . . . . . 64

4 O Oscilador Paramétrico Ótico $\quad 71$

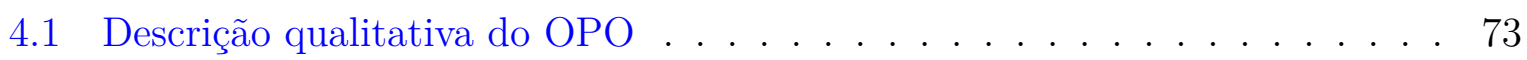

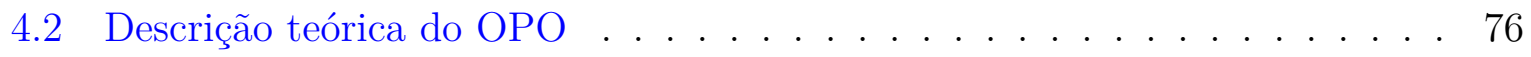

4.3 Soluções Estacionárias . . . . . . . . . . . . . . . . . . . . . 79

4.4 Flutuações Quânticas Linearizadas . . . . . . . . . . . . . . . . . . 81

4.5 Solução Matricial . . . . . . . . . . . . . . . . . . . . . . . . . 82

4.5.1 Aplicação do critério PPT . . . . . . . . . . . . . . . 85

4.6 Gaussianidade dos feixes produzidos pelo OPO acima do limiar . . . . . . 88

5 Resultados Experimentais $\quad 91$

5.1 Experimento . . . . . . . . . . . . . . . . . . 93

5.1.1 Calibração do shot-noise: Configuração 1 . . . . . . . . . . . . . . 100

5.1 .2 Calibração do shot-noise: Configuração 2 . . . . . . . . . . . . . . . 105

5.2 Medidas de emaranhamento tripartite . . . . . . . . . . . . . . 106

5.3 Morte súbita de emaranhamento . . . . . . . . . . . . . . . . . 112

5.3 .1 Caso tripartite . . . . . . . . . . . . . . . . . . 112

5.3 .2 Morte súbita bipartite . . . . . . . . . . . . . . . 116

5.4 Teste de gaussianidade . . . . . . . . . . . . . . . . . . . . 118

$\begin{array}{llr}6 & \text { Concluões e perspectivas } & 123\end{array}$ 


\section{Capítulo 1}

\section{Introdução}

Pesquisas em ótica quântica têm ganhado grande visibilidade devido ao interesse crescente por sistemas que, pelo menos em princípio, permitem processar, armazenar e realizar transferência de informação com altas velocidade, eficiência e segurança [Zeilinger 2007]. Dada a vasta utilização de sinais luminosos como ferramenta de comunicação, parece evidente utilizar a luz em protocolos quânticos, dada sua alta velocidade e baixa interação com o meio, sendo assim menos suscetível a perdas [Kimble 2008]. Naturalmente, os avanços tecnológicos atuais já requerem o entendimento de propriedades da teoria quântica, uma vez que sistemas usados para realizar o processamento e transmissão de informação se aproximam cada vez mais de limites impostos por princípios fundamentais da natureza.

Dentre as propriedades de sistemas quânticos o emaranhamento pode ser considerado como um dos principais focos de pesquisa dentro da física, principalmente por conta da sua importância no processamento de informação quântica [Braunstein 2005]. Reconhecido primeiramente por Einstein, Podolsky e Rosen (EPR) em 1935 [Einstein 1935], o emaranhamento era visto como uma prova da falta de compreensão da teoria quântica, levando a situações aparentemente absurdas como, por exemplo, a violação do princípio de localidade que deu origem à expressão "ação fantasmagórica à distância". Motivado pelo trabalho EPR, Schrödinger elaborou um trabalho independente [Schrödinger 1935] onde usou cunhou a expressão Verschränkung, traduzida do alemão para o inglês como entanglement, para enfatizar que as propriedades de duas partículas que formam um sistema composto não-fatorizável estão ligadas de modo inextricável. O emaranhamento surge no âmbito de sistemas compostos e é fruto de estados globais que não possuem fatorabilidade ou separabilidade.

O trabalho EPR teve como objetivo principal mostrar elementos que fariam da mecânica quântica uma teoria incompleta. A descrição de EPR envolvia um sistema composto 
por duas partículas que interagiram por um curto intervalo de tempo e depois se afastavam atingindo distâncias que cessavam qualquer tipo de interação. Após a separação, medidas efetuadas em cada uma das partes apresentariam correlações que aparentemente violariam as relações de incerteza de Heisenberg, tendo como consequência situações que poderiam desafiar o senso comum, dado que não haveria mais comunicação entre elas.

Bohr, no mesmo ano da publicação de EPR, argumentou que os estados propostos por Einstein estavam consistentes com a mecânica quântica e obedeciam o princípio da incerteza [Bohr 1935]. Para EPR, era estranho não poder atribuir valores para grandezas independentemente do processo de medida, um pensamento determinista. Somente em 1964 surgiu a possibilidade de colocar à prova o raciocínio EPR. John Bell, fazendo uso das asserções sobre realismo e localidade de EPR e assumindo que estes estavam corretos, mostrou ser possível colocar o problema do emaranhamento em um contexto experimental [Bell 1964]. Por meio de uma teoria baseada em variáveis ocultas locais, Bell mostrou que as probabilidades associadas aos resultados de medidas feitas em um sistema quântico emaranhado violavam desigualdades que deveriam ser obedecidas por um sistema que satisfizesse as hipóteses de realismo e localidade. Desta maneira, se comprovado, o emaranhamento surgiria como uma característica intrínseca da mecânica quântica.

Os primeiros experimentos que tentaram tirar o emaranhamento do campo das ideias surgiram ainda na década de 60 [Kocher 1967, Freedman 1972], no entanto somente em 1982 foi realizada a primeira comprovação experimental do fenômeno [Aspect 1982]. Porém, imperfeições experimentais tendem a mascarar as contradições existentes entre a teoria quântica e os conceitos de realidade e localidade propostos por EPR. Para isso, uma demonstração experimental rigorosa da violação das desigualdades de Bell deve preencher dois requisitos: primeiro, dois observadores separados devem possuir sistemas de detecção completamente independentes, de modo que possa ser descartada qualquer eventual comunicação entre os dois; por exemplo, no caso onde a análise é feita por polarização, as polarizadores de cada sistema devem ser alteradas de modo aleatório com uma velocidade tão rápida que nenhuma informação sobre o aparato de um observador possa viajar, durante o processo de medida, para o outro com velocidade inferior à velocidade da luz e, segundo, deve-se ter uma alta eficiência de detecção tal que os resultados não possam ser explicados em termos realistas locais; isto significa que a eficiência total de detecção deve ser maior que um determinado limiar, caso contrário o subconjunto de eventos detectados pode concordar com as previsões da mecânica quântica, embora o conjunto de todos os eventos satisfaça as desigualdades de Bell [Pearle 1970]. Experimentos que não cumpram essas exigências são ditos abertos a "loophole" de localidade e detecção [Simon 2003].

Após sua comprovação experimental, logo foi vislumbrada a aplicação de emaranhamento na troca de informação entre partes separadas por grandes distâncias, visando o aumento de velocidade e segurança no processo. Atualmente, o emaranha- 
mento é um ingrediente chave na implementação de protocolos de criptografia quântica [Ekert 1991, Silberhorn 2002], codificação densa [Bennett 1992, Li 2002] e teletransporte de estados quânticos [Bennett 1993, Furusawa 1998]. A comunidade científica, motivada por essas ideias, logo pensou em um aumento na capacidade de processamento de dados e armazenamento dessa informação, o que deu origem à Computação Quântica [Nielsen 2000]. Além disso, fora toda a motivação econômica existente, o emaranhamento por si só é algo interessante de ser estudado, pois está inserido no contexto de pesquisa básica, envolvendo questionamentos sobre os fundamentos da mecânica quântica.

No entanto, mesmo com todos os vislumbramentos, muito sobre emaranhamento continua desconhecido. Em geral, o emaranhamento possui estrutura complexa, difícil de ser identificada na maioria dos casos e sua evolução envolvendo processos de descoerência é algo não-trivial. Por estes motivos, são consideradas questões fundamentais no estudo do emaranhamento: (1) Como identificar de modo inequívoco um estado emaranhado, teórica e experimentalmente? (2) Como evitar o processo de descoerência e, caso seja inevitável, como revertê-lo? (3) Como caracterizar, controlar e quantificar o emaranhamento?. A literatura que tenta responder esses questionamentos é extensa [Horodecki R. 2007].

As respostas para esses questionamentos são importantes para desenvolver métodos que possibilitem realizar o armazenamento de informação utilizando emaranhamento em meios materiais, tais como átomos ou íons, além de permitir desenvolver protocolos de transmissão de informação sendo necessário, para este fim, o uso de meios pouco interagentes.

O Laboratório de Manipulação Coerente de Átomos e Luz (LMCAL) tem desenvolvido experimentos que podem contribuir para a elucidação dessas respostas. Em especial, o trabalho que será apresentado aqui se concentra na produção de estados emaranhados entre feixes intensos de luz. Para isso, é utilizado um Oscilador Paramétrico Ótico (OPO) triplamente ressonante acima do limiar de oscilação. Trata-se de uma fonte sintonizável que permite gerar campos emaranhados com comprimentos de onda distintos. Com esta fonte, realizamos a primeira medida de emaranhamento tripartite entre os feixes sinal, complementar e bombeio refletido produzidos pelo OPO. Esta é a primeira geração direta de emaranhamento entre mais de dois subsistemas em variáveis contínuas [Coelho 2009b].

Para verificar a existência de emaranhamento utilizamos o critério de Positividade sob Transposição Parcial (PPT) elaborado por Asher Peres [Peres 1996], demonstrado por Horodecki [Horodecki M. 1996] ser necessário e suficiente para sistemas com baixa dimensão, $(2 \times 2)$ e $(2 \times 3)$, descritos por variáveis discretas. A versão estendida para variáveis contínuas, feita por Simon [Simon 2000], mostra-se necessária e suficiente para estados gaussianos com partições do tipo $(1 \times 1)$. Em seguida, foi provado por Werner e Wolf [Werner 2001] que estas mesmas propriedades são válidas para sistemas analisados em partições do tipo $(1 \times(N-1)$, em que $N$ é o número total de subsistemas.

Após identificar o emaranhamento em nosso sistema, realizamos sua caracterização 
frente a perdas. Com este estudo, identificamos as regiões de parâmetros de funcionamento do sistema que apresentam limitações para eventuais aplicações [Coelho 2009b, Barbosa 2010a]. Além disto, encontramos fortes evidências de características análogas às encontradas em estados $G H Z$ e $W$, até o momento demonstradas experimentalmente somente no âmbito de variáveis discretas[Dür 2000].

Os principais resultados desta tese são: a medida do emaranhamento tripartite, caracterização experimental do emaranhamento bipartite e tripartite frente a perdas e estudo experimental da estatística gaussiana das flutuações dos campos produzidos por um OPO triplamente ressonante acima do limiar de oscilação.

No Capítulo 2, apresentaremos conceitos fundamentais para o entendimento dos resultados. Iniciaremos com uma descrição breve do campo no contexto clássico e quântico, introduzindo as quadraturas amplitude e fase, bem como as flutuações a elas associadas, organizadas posteriormente em uma matriz de covariâncias. Apresentaremos também detalhes sobre o processo de fotodetecção das quadraturas dos campos, explicitando os modos das frequências efetivamente envolvidas no processo. Em seguida, falaremos sobre o shot-noise, a referência para os ruídos do campo. Por fim, discutiremos as técnicas de detecção homodina e auto-homodina, empregadas para a medida das flutuações das quadraturas, indicando quais informações podem ser extraídas por cada técnica.

O emaranhamento será discutido no Capítulo 3. Iniciaremos apresentando as primeiras reflexões acerca do emaranhamento, deixando claro de antemão que não se trata de uma descrição completa. Daremos a definição formal de estados emaranhados e explicaremos dois critérios de separabilidade, o critério de Duan [Duan 2000], ou critério de soma de variâncias, e o critério de Positividade sob Transposição Parcial (PPT) [Simon 2000, Peres 1996].

A teoria do OPO será apresentada no capítulo 4, seguindo de perto a descrição exposta nas referências [Cassemiro 2008, Villar 2007b, Martinelli 2002]. Além de identificar o emaranhamento produzido em nosso sistema, estamos interessados em estudar o efeito das perdas sobre os campos, algo que deve ser feito com um critério que seja necessário e suficiente para a determinação do emaranhamento. O critério PPT preenche este requisito para estados gaussianos analisados em bipartições do tipo $1 \times(N-1)$. Por conta disso, faz-se necessário mostrar que a estatística dos estados produzidos pelo OPO é gaussiana. Os elementos necessários para estudar a estatística das flutuações dos campos também são descritos neste capítulo.

Os resultados experimentais são expostos no capítulo 5. Além dos resultados principais, descrevemos o nosso aparato experimental, indicando quais os novos elementos 
inseridos, e as melhorias implementadas nos já existentes desde a elaboração de outros trabalhos de tese do grupo [Cassemiro 2008, Villar 2007b].

Finalmente, no capítulo 6, apresentamos nossas conclusões e perspectivas. 


\section{Capítulo 2}

\section{Conceitos fundamentais}

We all know what is light; but it is not easy tell what it is.

Samuel Johnson (1709-1784) Queremos saber, Queremos saber, todos queremos saber. G. Gil (1976)

O objetivo deste capítulo é deixar o leitor a par da notação usada ao longo trabalho, bem como introduzir alguns conceitos fundamentais. Definiremos as quadraturas amplitude e fase do campo eletromagnético no contexto clássico e quântico e as flutuações associadas a elas. Todas as medidas de potência de ruído são normalizadas pelo ruído quântico padrão ou shot-noise ${ }^{1}$, que desempenha um papel fundamental na determinação de características intrinsecamente quânticas. Mostraremos como é realizada a medida do shot-noise $^{2}$ utilizando a técnica de detecção homodina que consiste em misturar o campo de interesse com um campo intenso onde serão projetadas as flutuações que desejamos acessar.

Com o uso desta mesma técnica, faremos o tratamento do processo de fotodetecção partindo de primeiros princípios, deixando claro quais modos do campo são realmente medidos, reinterpretando as quadraturas amplitude e fase usualmente utilizadas em todos os trabalhos do grupo até hoje. Dado que os estados produzidos em nosso sistema são gaussianos, definiremos a matriz de covariâncias para as quadraturas e sua relação com o operador densidade e função de Wigner.

Finalmente, descreveremos a técnica auto-homodina que tira proveito da propriedade dispersiva de cavidades óticas vazias. Com a análise da fotodetecção é possível ver que

\footnotetext{
${ }^{1}$ Dado o uso recorrente do termo em inglês ao longo da tese, optamos por não utilizar o vocábulo em itálico.

${ }^{2}$ Ao longo do texto usaremos de forma indiscriminada os termos shot-noise e ruído quântico padrão para as flutuações de estados coerentes.
} 
a auto-homodinagem pode trazer mais informações no domínio da frequência em comparação com a técnica de detecção homodina. O tratamento teórico sobre estas questões, da forma como está apresentado aqui, foi elaborado pelo Prof. Alessandro Villar ${ }^{3}$ e está descrito em sua série de materiais elaborados para discussão interna do grupo.

A principal razão para o uso das cavidades óticas é a diferença entre os comprimentos de onda dos campos existentes em nosso experimento, pois não há disponibilidade de um oscilador local para cada feixe a fim de realizar a detecção homodina. A teoria completa da detecção auto-homodina é encontrada nas referências [Villar 2008, Villar 2007b], sendo abordado aqui somente o fundamental para o entendimento da análise de dados.

O tratamento dado ao campo de radiação livre é baseado na referência [Piza 2010], de onde partimos para introduzir as quadraturas amplitude e fase, tanto no contexto clássico quanto quântico. Para mais detalhes sobre conceitos fundamentais em ótica quântica são recomendadas as referências [Leonhardt 2010, Fox 2000, Bachor 1998].

\subsection{Quadraturas do campo: tratamento clássico}

Consideremos uma região do espaço onde não há densidade de cargas e correntes, ou seja, uma região livre de fontes. O eletromagnetismo clássico, dentro dessa região, é governado pelas equações de Maxwell com as relações constitutivas para o vácuo.

Para os nossos propósitos, é conveniente adotar a condição de calibre (ou "gauge") de Coulomb, introduzindo o potencial vetor $\vec{A}(\vec{r}, t)$ com divergência nula, $\nabla \cdot \vec{A}(\vec{r}, t)=0$. Nessa condição, assume-se, sem perder em generalidade, que o potencial escalar $\Phi(\vec{r}, t)$ é nulo. Assim, os campos elétrico e magnético passam a ser representados em termos de um potencial vetor através das relações

$$
\begin{aligned}
\vec{E}(\vec{r}, t) & =-\frac{1}{c} \frac{\partial \vec{A}(\vec{r}, t)}{\partial t} \mathrm{e} \\
\vec{B}(\vec{r}, t) & =\nabla \times \vec{A}(\vec{r}, t),
\end{aligned}
$$

onde $c=1 / \sqrt{\mu_{0} \epsilon_{0}}$ é a velocidade da luz no vácuo, $\mu_{0}$ a permeabilidade e $\epsilon_{0}$ a permissividade do espaço livre.

Verifica-se a partir das equações de Maxwell que o potencial vetor satisfaz a equação

$$
\left[\nabla^{2}-\frac{1}{c^{2}} \frac{\partial^{2}}{\partial t^{2}}\right] \vec{A}(\vec{r}, t)=0
$$

que tem como solução geral

$$
\vec{A}(\vec{r}, t)=\sum_{\vec{k} \ell} \vec{A}_{\vec{k} \ell}(\vec{r}, t), \quad \text { com } \quad \vec{A}_{\vec{k} \ell}(\vec{r}, t)=\sqrt{\frac{4 \pi c^{2}}{L^{3}}} \vec{\epsilon}_{\vec{k}, \ell} \vec{q}_{k, \ell}(t) e^{i \vec{k} \cdot \vec{r}} .
$$

\footnotetext{
${ }^{3}$ E-mail: villar@df.ufpe.br .
} 
Essa solução é obtida considerando condições periódicas de contorno num volume $L^{3}$ do espaço livre. Vemos então que o potencial vetor $\vec{A}(\vec{r}, t)$ é escrito como uma soma de modos $\vec{q}_{\vec{k} \ell}(t)$, rotulados pelo vetor $\vec{k}$ e pelo índice $\ell=1,2$ que definem, respectivamente, a direção de propagação e a polarização do campo, cuja orientação é determinada pelo vetor $\vec{\epsilon}_{\vec{k}, \ell}$ que descreve duas orientações perpendiculares entre si e ao vetor $\vec{k}$.

Devido à linearidade da equação de onda, ao substituir a equação (2.1.3) em (2.1.2), cada modo do campo deve satisfazer

$$
\frac{d^{2} q_{\vec{k} \ell}(t)}{d t^{2}}+\omega_{k}^{2} q_{\vec{k} \ell}(t)=0
$$

com a frequência $w_{\vec{k}}=|\vec{k}| c$. Essas funções são em geral complexas e fornecem uma forma simples para a condição de realidade que deve ser satisfeita pelo potencial vetor,

$$
\sum_{\vec{k} \ell} \vec{A}_{\vec{k} \ell}(\vec{r}, t)=\sum_{\vec{k} \ell} \vec{A}_{\vec{k} \ell}^{*}(\vec{r}, t)
$$

Desta equação decorre que

$$
\sum_{\vec{k} \ell} \vec{\epsilon}_{\vec{k}, \ell} \vec{q}_{k, \ell}(t) e^{i \vec{k} \cdot \vec{r}}=\sum_{\vec{k} \ell} \vec{\epsilon}_{\vec{k}, \ell} \vec{q}_{k, \ell}^{*}(t) e^{-i \vec{k} \cdot \vec{r}}=\sum_{\vec{k} \ell} \vec{\epsilon}_{-\vec{k}, \ell} \vec{q}_{-k, \ell}^{*}(t) e^{i \vec{k} \cdot \vec{r}} .
$$

Os vetores que definem a polarização seguem a convenção $\vec{\epsilon}_{\vec{k}, \ell}=\vec{\epsilon}_{-\vec{k}, \ell}$ [Piza 2010], que, juntamente com a independência linear das ondas planas, reduzem a condição de realidade a $\vec{q}_{k, \ell}(t)=\vec{q}_{-k, \ell}^{*}(t)$.

Vemos pela equação (2.1.4) que o valor o campo elétrico monocromático (de frequência $\left.\omega_{0}\right)$ pode ser escrito como,

$$
q_{\vec{k}_{0}, \ell} \equiv E(t)=\alpha e^{-i w_{0} t}+\alpha^{*} e^{i w_{0} t} .
$$

Suprimimos as grandezas que definem a dimensão do campo e definimos a amplitude complexa $\alpha=\alpha_{0} e^{-i \varphi}$ com uma fase $\varphi$ arbitrária. Expandindo as exponenciais podemos escrever a amplitude do campo em função de dois termos em quadratura,

$$
\begin{gathered}
E(t)=X \cos \left(\omega_{0} t\right) \quad+\quad Y \operatorname{sen}\left(\omega_{0} t\right), \operatorname{com} \\
X=2 \operatorname{Re}(\alpha)=\alpha+\alpha^{*} \quad \text { e } \quad Y=2 \operatorname{Im}(\alpha)=-i\left(\alpha-\alpha^{*}\right) .
\end{gathered}
$$

Em uma situação física real, os campos eletromagnéticos, e por consequência suas quadraturas, estão sujeitos a flutuações que podem ser originadas por efeitos clássicos ou serem de natureza quântica. Para explicitar as grandezas importantes na descrição do campo, representamos o mesmo em um diagrama de fasores conforme ilustrado na figura 2.1(a), cujos eixos são dados por $X$ e $Y$. A amplitude $|\alpha|$ do campo é dada pelo módulo do fasor e sua fase definida como $\varphi=\arctan (Y / X)$. Incluímos na representação as quadraturas amplitude $\delta p$ e fase $\delta q$ da flutuação $\delta \alpha$, comparadas às quadraturas $\delta X \mathrm{e}$ $\delta Y$ tomadas com uma referência de fase arbitrária. 


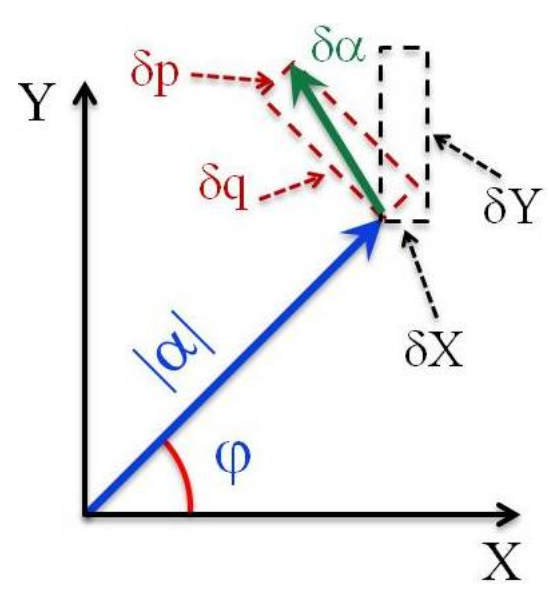

(a)

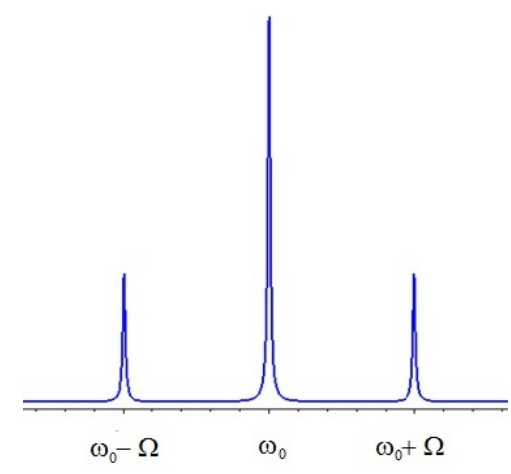

(b)

Figura 2.1: (a) Representação do campo em um diagrama de fasores. O comprimento do fasor partindo da origem representa o valor médio do campo. (b) Após a modulação de amplitude, três frequências, $\omega_{0}$ e $\left(\omega_{0} \pm \Omega\right)$, passam a contribuir para a intensidade do campo. A frequência central é chamada portadora e as demais denominadas bandas laterais.

Conforme será tratado aqui, as informações sobre tais flutuações se fazem notar em bandas laterais, frequências específicas geradas no entorno da frequência principal do campo, a portadora. Para ver como isso ocorre, vamos seguir a abordagem apresentada em [Bachor 1998]. Considerando nula a fase arbitrária, $\varphi=0$, verifiquemos o que ocorre quando inserimos no campo $E(t)$ uma pequena modulação de amplitude com frequência $\Omega$ :

$$
\begin{aligned}
E(t) & =\alpha_{0}\{1-M[1-\cos (\Omega t)]\}\left(e^{-i w_{0} t}+e^{i w_{0} t}\right) \\
& =2(1-M) \alpha_{0} \cos \left(w_{0} t\right)+2 M \alpha_{0}\left\{\cos \left[\left(w_{0}+\Omega\right) t\right]+\cos \left[\left(w_{0}-\Omega\right) t\right]\right\}
\end{aligned}
$$

de onde se veem as contribuições das três frequências: portadora $w_{0}$ e bandas laterais $w_{0} \pm \Omega$, ilustradas na figura 2.1(b). A constante $M$ está associada à amplitude do sinal de modulação. É importante notar que, mesmo sendo a amplitude de modulação muito pequena $(M \ll 1)$, os efeitos das bandas laterais são perceptíveis graças ao fato de suas amplitudes serem proporcionais à amplitude da portadora. Vê-se isso mais claramente quando calculamos a intensidade do campo $I(t)=|E(t)|^{2}$ para $M \ll 1$,

$$
\begin{aligned}
I(t) & =\left\{2(1-M) \alpha_{0} \cos \left(w_{0} t\right)+2 M \alpha_{0}\left[\cos \left[\left(w_{0}+\Omega\right) t\right]+\cos \left[\left(w_{0}-\Omega\right) t\right]\right]\right\}^{2} \\
& \longrightarrow 4 \alpha_{0}^{2} \cos ^{2}\left(w_{0} t\right)-8 M \alpha_{0}^{2} \cos ^{2}\left(w_{0} t\right)[1-2 \cos (\Omega t)] \\
& =I_{0}-2 I_{0} M[1-\cos (\Omega t)]
\end{aligned}
$$

em que $I_{0}$ é a intensidade do campo sem modulação. Vê-se, então, que a intensidade da banda lateral é proporcional à intensidade da portadora central. 
Em uma descrição geral, as flutuações existentes nos campos se estendem por todo o espectro de ruído, e a escolha da componente de frequência na qual desejamos estudar as flutuações do campo se dá pelo uso de dispositivos eletrônicos utilizados para a demodulação do ruído [Bachor 1998]. A frequência escolhida para analisar as flutuações é denominada, por esta razão, frequência de análise, e podem ser interpretadas como as modulações que acabamos de descrever. É também necessário entender a relação existente entre as quadraturas e as flutuações medidas. Portanto, definimos $X$ e $Y$ de forma mais geral,

$$
X_{\theta}=\alpha(t) e^{-i \theta}+\alpha(t)^{*} e^{i \theta} \quad \text { e } \quad Y_{\theta}=-i\left[\alpha(t) e^{-i \theta}-\alpha(t)^{*} e^{i \theta}\right]
$$

onde a dependência temporal surge por conta das modulações existentes e $\theta$ incluída como uma referência de fase que pode ser manipulada experimentalmente, permitindo girar as quadraturas com relação ao referencial do campo. Desse modo, se $\theta=\varphi$ a quadratura $X_{\varphi}$ está alinhada com o vetor do campo e qualquer flutuação nessa quadratura é interpretada como flutuação de amplitude. Vemos este fato adicionando à amplitude complexa $\alpha(t)$ um termo de flutuação $\delta \alpha(t)$, de modo que se $\delta \alpha(t) \ll \alpha(t)$, a intensidade do campo passa a ser escrita da seguinte maneira:

$$
\begin{aligned}
I(t) & =\langle I\rangle+\delta I(t)=\alpha(t) \alpha^{*}(t) \\
& =[\alpha+\delta \alpha(t)]\left[\alpha^{*}+\delta \alpha^{*}(t)\right] \\
& =\alpha_{0}^{2}+\alpha_{0}\left[\delta \alpha^{*}(t) e^{-i \varphi}+\delta \alpha(t) \mathrm{e}^{i \varphi}\right]+\mathcal{O}\left[\delta \alpha(t)^{2}\right],
\end{aligned}
$$

o que fornece para a flutuação de intensidade

$$
\delta I(t) \cong\left|\alpha_{0}\right|\left[\delta \alpha^{*}(t) e^{-i \varphi}+\delta \alpha(t) e^{i \varphi}\right]=\left|\alpha_{0}\right| \delta X_{\varphi}(t) .
$$

Vemos então que a flutuação da quadratura $X_{\varphi}(t)$ está diretamente relacionada com a flutuação de amplitude do campo, enquanto $Y_{\varphi}(t)$ se relaciona com as flutuações de fase. Ao longo do trabalho é usada a seguinte convenção: $p(t) \equiv X_{\varphi}(t)$ e $q(t) \equiv Y_{\varphi}(t)$ para definir as quadraturas amplitude e fase, respectivamente. Note que este tratamento é feito aqui no domínio temporal e tem como objetivo relacionar as flutuações de intensidade do campo com suas quadraturas. Sendo assim, a sua relação com as bandas laterais se torna explícita quando o levamos para o domínio da frequência, algo usualmente realizado com a aplicação de transformadas de Fourier nas expressões (2.1.12), assunto que será tratado mais à frente.

Vejamos a seguir como essas grandezas são apresentadas no domínio quântico.

\subsection{Quadraturas do campo: tratamento quântico}

No tratamento clássico do campo eletromagnético em uma condição de calibre de Coulomb, o potencial vetor $\vec{A}(\vec{r}, t)$ obedece a equação de onda (2.1.2), de modo que sua 
solução geral possibilita escrevê-lo como uma soma infinita de modos espaço-temporais. Em especial, os modos espaciais se comportam como um conjunto de osciladores harmônicos, dada sua descrição pela equação (2.1.4). Portanto, a quantização do campo de radiação livre se dá por meio da quantização desse conjunto de osciladores. Fazemos isso reinterpretando a amplitude $q_{\vec{k}, \ell}$ e o momento $p_{\vec{k}, \ell} \equiv \frac{d}{d t} q_{\vec{k}, \ell}$ como operadores hermitianos que satisfazem as relações de comutação

$$
\left[\hat{q}_{\vec{k}, \ell}, \hat{p}_{\vec{k}^{\prime}, \ell^{\prime}}\right]=i \hbar \delta_{\vec{k}, \vec{k}^{\prime}} \delta_{\ell, \ell^{\prime}}, \quad\left[\hat{q}_{\vec{k}, \ell}, \hat{q}_{\vec{k}^{\prime}, \ell^{\prime}}\right]=0 \quad \text { e } \quad\left[\hat{p}_{\vec{k}, \ell}, \hat{p}_{\vec{k}^{\prime}, \ell^{\prime}}\right]=0
$$

Seguindo o mesmo raciocínio utilizado na quantização de um oscilador harmônico, é conveniente definir os operadores adimensionais não-hermitianos de criação $\left(\hat{a}^{\dagger}\right)$ e aniquilação (â) através das relações

$$
\begin{aligned}
\hat{q}_{\vec{k}, \ell} & =\sqrt{\frac{\hbar}{2 w_{k}}}\left(\hat{a}_{\vec{k}, \ell} e^{-i w_{k} t}+\hat{a}_{-k, \ell}^{\dagger} e^{i w_{k} t}\right) \quad \mathrm{e} \\
\hat{p}_{\vec{k}, \ell} & =i \sqrt{\frac{\hbar w_{k}}{2}}\left(\hat{a}_{\vec{k}, \ell}^{\dagger} e^{i w_{k} t}-\hat{a}_{-k, \ell} e^{-i w_{k} t}\right)
\end{aligned}
$$

$\operatorname{com}\left[\hat{a}_{\vec{k}, \ell}, \hat{a}_{\vec{k}^{\prime}, \ell^{\prime}}^{\dagger}\right]=\delta_{\vec{k}, \vec{k}^{\prime}} \delta_{\ell, \ell^{\prime}}$, dada as condições de realidade do potencial vetor, $\hat{q}_{\vec{k}, \ell}=\hat{q}_{-k, \ell}^{\dagger}$ e $\hat{p}_{\vec{k}, \ell}=\hat{p}_{-k, \ell}^{\dagger}$. Note que isto garante ainda que $\hat{p}_{\vec{k}, \ell}$ e $\hat{q}_{\vec{k}, \ell}$ sejam operadores hermitianos. Estes objetos quânticos representam osciladores harmônicos com frequência $w_{\vec{k}}=|\vec{k}| c$, cuja analogia com o caso clássico pode ser feita pela associação da amplitude complexa $\alpha$ com o operador de aniquilação, $\alpha \rightarrow \hat{a}$, conforme discutido em [Cohen-Tannoudji 2004] na passagem da descrição clássica para a quântica.

A fim de simplificar a notação são introduzidas as equivalências

$$
\lambda \equiv\{\vec{k}, \ell\}, \quad-\lambda \equiv\{\overrightarrow{-k}, \ell\} \quad \text { e } \quad w_{k} \equiv w_{\lambda}=w_{-\lambda},
$$

de forma que $\lambda$ passa a representar um modo do campo. Feito isso, o potencial vetor e o campo elétrico quantizados têm suas expressões dadas por

$$
\begin{aligned}
\hat{\mathbf{A}}(\vec{r}, t) & =\sqrt{\frac{1}{L^{3}}} \sum_{\lambda} \sqrt{\frac{2 \pi \hbar c}{k}} \vec{\epsilon}_{\lambda}\left[\hat{a}_{\lambda} e^{i\left(\vec{k} \cdot \vec{r}-w_{\lambda} t\right)}+\hat{a}_{\lambda}^{\dagger} e^{-i\left(\vec{k} \cdot \vec{r}-w_{\lambda} t\right)}\right], \\
\hat{\mathbf{E}}(\vec{r}, t) & =\hat{\mathbf{E}}^{(+)}(\vec{r}, t)+\hat{\mathbf{E}}^{(-)}(\vec{r}, t),
\end{aligned}
$$

sendo

$$
\hat{\mathbf{E}}^{(+)}(\vec{r}, t)=i \sum_{\lambda} \sqrt{\frac{2 \pi \hbar w_{\lambda}}{L^{3}}} \vec{\epsilon}_{\lambda} \hat{a}_{\lambda} e^{i\left(\vec{k} \cdot \vec{r}-w_{\lambda} t\right)} \quad \text { e } \quad \hat{\mathbf{E}}^{(-)}(\vec{r}, t)=\left[\hat{\mathbf{E}}^{(+)}(\vec{r}, t)\right]^{\dagger} .
$$

É válido lembrar que essas são expressões obtidas na representação de Heisenberg, sendo escritas na representação de Schrödinger para $t=0$ [Piza 2010]. 
Como a energia é uma grandeza extensiva, a hamiltoniana do campo livre é dada pela soma das hamiltonianas dos osciladores que o compõem,

$$
\hat{H}=\sum_{\lambda} \hat{H}_{\lambda}=\sum_{\lambda} \hbar \omega_{\lambda}\left(\hat{a}_{\lambda}^{\dagger} \hat{a}_{\lambda}+\frac{1}{2}\right)
$$

com autovetores e autovalores dados por

$$
\left|n_{\lambda}\right\rangle \equiv\left|n_{1}\right\rangle \otimes\left|n_{2}\right\rangle \otimes \ldots \otimes\left|n_{\lambda}\right\rangle \otimes \ldots \quad \text { e } \quad E_{n_{\lambda}}=\sum_{\lambda} \hbar \omega_{\lambda}\left(n_{\lambda}+\frac{1}{2}\right)
$$

onde o autoestado $\left|n_{\lambda}\right\rangle$ é dado pelo produto dos autoestados individuais de cada modo.

Nessa descrição, vemos que $\hat{q}_{\vec{k}, \ell}$ assume o papel análogo ao da posição do oscilador harmônico e sendo correspondente à amplitude do modo $\{\vec{k}, \ell\}$ do potencial vetor $\hat{\mathbf{A}}(\vec{r}, t)$, enquanto $\hat{p}_{\vec{k}, \ell}$ desempenha o papel de momento, proporcional à amplitude do campo elétrico $\hat{\mathbf{E}}(\vec{r}, t)$.

De forma semelhante ao tratamento clássico de um campo monocromático de frequência $\omega_{0}$ (equação 2.1.12), somos levados à definição dos operadores de quadratura

$$
\hat{X}_{\theta}(t)=\hat{a}(t) e^{-i \theta}+\hat{a}^{\dagger}(t) e^{i \theta} \quad \text { e } \quad \hat{Y}_{\theta}(t)=-i\left[\hat{a}(t) e^{-i \theta}-\hat{a}^{\dagger}(t) e^{i \theta}\right],
$$

onde usamos evolução livre dos operadores de criação e aniquilação calculada pela equação de Heisenberg que implica $\hat{a}(t)=\hat{a} e^{-i \omega_{0} t}$.

Em condições reais, quando falamos de um campo monocromático de frequência $\omega_{0}$ estamos nos referindo a um laser cuja frequência está definida dentro de uma faixa estreita conhecida como largura de linha, onde a frequência $\omega_{0}$ que o caracteriza ocupa a posição central desta faixa. Assim, na expressão (2.2.7), a contribuição do fator $\sqrt{\omega_{\lambda}}$ é quase completamente dada por $\sqrt{\omega_{0}}$. Dessa forma, é conveniente considerar os operadores acima como sendo uma combinação de operadores no domínio da frequência,

$$
\hat{a}(t)=\int_{0}^{\infty} d \omega \hat{a}(\omega) e^{-i \omega t} \quad \text { e } \quad \hat{a}^{\dagger}(t)=\int_{0}^{\infty} d \omega \hat{a}^{\dagger}(\omega) e^{i \omega t}
$$

Visando tornar explícitas as frequências no entorno da portadora de frequência $\omega_{0}$, podemos reescrever as equações acima em termos dos operadores lentamente variáveis, $\hat{a}(t)=\tilde{a}(t) e^{-i \omega_{0} t}$ e $\hat{a}^{\dagger}(t)=\tilde{a}^{\dagger}(t) e^{i \omega_{0} t}$, o que fornece

$$
\tilde{a}(t)=\int_{0}^{\infty} d \omega \hat{a}(\omega) e^{-i\left(\omega-\omega_{0}\right) t} \quad e \quad \tilde{a}^{\dagger}(t)=\int_{0}^{\infty} d \omega \hat{a}^{\dagger}(\omega) e^{i\left(\omega-\omega_{0}\right) t} .
$$

Agora, realizamos a mudança de variáveis $\omega \longrightarrow w_{0}+\Omega$ de modo a obtermos

$$
\int_{0}^{\infty} d \omega e^{-i\left(\omega-\omega_{0}\right) t} \hat{a}(\omega) \longrightarrow \int_{-\omega_{0}}^{\infty} d \Omega e^{-i \Omega t} \hat{a}\left(\omega_{0}+\Omega\right) \approx \int_{-\infty}^{\infty} d \Omega e^{-i \Omega t} \hat{a}(\Omega)
$$

onde foi usada uma notação mais compacta, a saber $\hat{a}\left(\Omega+\omega_{0}\right) \equiv \hat{a}(\Omega)$. A alteração do limite de integração $\omega_{0} \rightarrow-\infty$ pode ser feita uma vez que $\Omega \ll \omega_{0}$ e principalmente pelo fato do campo considerado monocromático ter uma largura de linha estreita, de forma 
que as frequências fora dessa largura têm contribuições nulas para o sinal de fotodetecção, como será visto posteriormente. Tendo em mente as condições periódicas de contorno e a continuidade do espectro, sabemos que a relação de comutação entre os operadores de criação e aniquilação é dada por $\left[\hat{a}(\Omega), \hat{a}^{\dagger}\left(\Omega^{\prime}\right)\right]=\delta\left(\Omega-\Omega^{\prime}\right)$. Isso permite mostrar que a relação de comutação entre as quadraturas é escrita como

$$
\left[\hat{X}_{\theta}(t), \hat{Y}_{\theta}\left(t^{\prime}\right)\right]=2 i \delta\left(t-t^{\prime}\right)
$$

Resta-nos agora relacionar os operadores citados com grandezas medidas no laboratório. Podemos definir o operador de aniquilação do campo como soma do seu valor médio com um termo de flutuação, $\hat{a}(t)=\alpha+\delta \hat{a}(t)$, com $\alpha \equiv\langle\hat{a}(t)\rangle=\alpha_{0} e^{i \varphi}$, de modo que para situações onde a flutuação do campo é muito menor que seu valor médio, $\delta \hat{I}(t) \ll\langle\hat{I}\rangle$, obtemos

$$
\begin{aligned}
\hat{I}(t) & =\langle\hat{I}\rangle+\delta \hat{I}(t)=\hat{a}^{\dagger}(t) \hat{a}(t) \\
& =\left[\alpha^{*}+\delta \hat{a}^{\dagger}(t)\right][\alpha+\delta \hat{a}(t)] \\
& \cong\left|\alpha_{0}\right|^{2}+\left|\alpha_{0}\right|\left[\delta \hat{a}(t) e^{-i \varphi}+\delta \hat{a}^{\dagger}(t) e^{i \varphi}\right] \\
& \rightarrow \delta \hat{I}(t)=\left|\alpha_{0}\right| \delta \hat{X}_{\varphi},
\end{aligned}
$$

Mais uma vez, vemos que as flutuações de intensidade do campo são proporcionais às flutuações da quadratura $\delta \hat{X}_{\varphi}$. Continuaremos usando a convenção anterior $\hat{p}(t) \equiv \hat{X}_{\varphi}(t)$ e $\hat{q}(t) \equiv \hat{Y}_{\varphi}(t)$, para especificar as quadraturas amplitude e fase do campo.

Assim como na descrição clássica, as bandas laterais contêm toda a informação sobre as flutuações e estatística dos campos. Isso posto, é conveniente tratar os operadores no domínio da frequência, algo usualmente feito realizando a transformada de Fourier das expressões (2.2.10), obtendo

$$
\hat{X}_{\theta}(\Omega)=\hat{a}(\Omega) e^{-i \theta}+\hat{a}^{\dagger}(-\Omega) e^{i \theta} \quad \text { e } \hat{Y}_{\theta}(\Omega)=-i\left[\hat{a}(\Omega) e^{-i \theta}-\hat{a}^{\dagger}(-\Omega) e^{i \theta}\right],
$$

que obedecem às relações

$$
\left[\hat{X}_{\theta}(\Omega)\right]^{\dagger}=\hat{X}_{\theta}(-\Omega) \quad e \quad\left[\hat{Y}_{\theta}(\Omega)\right]^{\dagger}=\hat{Y}_{\theta}(-\Omega)
$$

obtidas da condição de hermiticidade dos operadores no domínio temporal. Deduz-se ainda que, dada a relação de comutação entre os operadores de criação e aniquilação, deve ser satisfeita a relação

$$
\left[\hat{X}_{\theta}(\Omega), \hat{Y}_{\theta}\left(\Omega^{\prime}\right)\right]=4 i \pi \delta\left(\Omega-\Omega^{\prime}\right)
$$

Contudo, neste ponto surge um problema conceitual importante: os operadores $\hat{p}(\Omega)=$ $\hat{X}_{\varphi}(\Omega)$ e $\hat{q}(\Omega)=\hat{Y}_{\varphi}(\Omega)$ atuam em modos legítimos do campo? Dito de outra forma, que modos do campo são descritos por estes operadores? Trata-se de uma pergunta pertinente, 
dado que nas expressões (2.2.16) esses operadores aparecem como uma combinação de operadores de criação e aniquilação dependentes de frequências distintas, $\hat{a}(\Omega)$ e $\hat{a}^{\dagger}(-\Omega)$. Além disso, as relações (2.2.17) mostram que $\hat{p}(\Omega)$ e $\hat{q}(\Omega)$ são operadores não hermitianos.

Para dar respostas a essas perguntas, definiremos o estado de nosso interesse e sua representação em uma matriz de covariâncias. Por fim, analisaremos a relação entre médias de grandezas tomadas no estado descrito pelo operador densidade e as médias obtidas no espaço de fase da função de Wigner.

\subsection{Estados gaussianos e matriz de covariâncias}

Devido à sua particular importância em experimentos em ótica e informação quântica, os estados gaussianos têm sido amplamente estudados, principalmente por permitirem um tratamento matemático mais simples no domínio de variáveis contínuas (CV) [Adesso 2006]. Estes estados são de interesse fundamental no desenvolvimento do trabalho que será apresentado. O estudo do nosso sistema é realizado no espaço de fase, sendo a função de Wigner a representação escolhida.

Dentre representações bem conhecidas que descrevem completamente o sistema no espaço de fase [Walls 1995, Scully 1997, Gardiner 1991], a função de Wigner é habitualmente chamada de distribuição de quasi-probabilidade, por conta de características que vão de encontro à definição de distribuições clássicas, como a possibilidade de valores negativos, por exemplo. Assim como as demais distribuições, a função de Wigner $W(p, q)$ representa completamente um sistema quântico descrito pelas variáveis canônicas $p$ e $q$, porém possui um caráter mais intuitivo, pois suas distribuições marginais correspondem exatamente às probabilidades de quadraturas no espaço de fase, por exemplo, $\int_{-\infty}^{\infty} W(p, q) d p$ e $\int_{-\infty}^{\infty} W(p, q) d q$ são as probabilidades associadas às grandezas $q$ e $p$, respectivamente.

Na referência [Leonhardt 1997], pode ser encontrada uma abordagem bastante didática sobre a função de Wigner, bem como a demonstração da sua forma mais conhecida:

$$
W(p, q)=\frac{1}{\pi} \int_{-\infty}^{\infty} d q^{\prime} \exp \left(i p q^{\prime}\right)\left\langle q-q^{\prime}|\hat{\rho}| q+q^{\prime}\right\rangle
$$

que aparece pela primeira vez no trabalho de Wigner sobre equilíbrio termodinâmico [Wigner 1932]. Vê-se então que existe uma relação direta entre a função $W(q, p)$ e o operador densidade $\hat{\rho}$ do sistema [Cohen-Tannoudji 1977], justificando o fato de termos toda a informação sobre o sistema contida nesta função.

Para nossos propósitos, é interessante notar que a função de Wigner também está diretamente relacionada com a matriz de covariâncias. Esta contém todas as informações sobre os momentos de segunda ordem das variáveis do sistema. Por exemplo, um campo com frequência ótica $\omega$ é representado pelos operadores de quadratura amplitude $\hat{p}_{\omega}$ e fase $\hat{q}_{\omega}$. Estes operadores obedecem às relações de comutação $\left[\hat{p}_{\omega}, \hat{q}_{\omega^{\prime}}\right]=2 i \delta\left(\omega-\omega^{\prime}\right)$ 
e se relacionam com os operadores de criação $\hat{a}_{\omega}^{\dagger}$ e aniquilação $\hat{a}_{\omega}$, os quais satisfazem $\left[\hat{a}_{\omega}, \hat{a}_{\omega^{\prime}}^{\dagger}\right]=2 i \delta\left(\omega-\omega^{\prime}\right), \hat{a}_{\omega}=\hat{p}_{\omega}+i \hat{q}_{\omega}$.

A fim de descrever um sistema composto por $\mathrm{N}$ campos monocromáticos, cada um representado por um modo, ordenamos os operadores $\hat{p}$ e $\hat{q}$ a eles associados em um vetor coluna $\hat{\mathcal{Z}}=\left(\hat{p}_{\omega_{1}}, \hat{q}_{\omega_{1}}, \hat{p}_{\omega_{2}}, \hat{q}_{\omega_{2}}, \ldots, \hat{p}_{\omega_{N}}, \hat{q}_{\omega_{N}}\right)$, onde $w_{i}$ é a frequência do $i$-ésimo modo. Em seguida, definimos a matriz de covariâncias

$$
\mathbf{V}=\frac{1}{2}\left\langle\left\{\hat{\mathcal{Z}}, \hat{\mathcal{Z}}^{\mathcal{T}}\right\}\right\rangle=\frac{1}{2}\left(\left\langle\hat{\mathcal{Z}} \cdot \hat{\mathcal{Z}}^{\mathcal{T}}\right\rangle+\left\langle\hat{\mathcal{Z}}^{\mathcal{T}} \cdot \hat{\mathcal{Z}}\right\rangle\right)
$$

onde a média é tomada em um estado que descreve o sistema quântico total. A relação entre a matriz de covariâncias e a função de Wigner se dá por meio do cálculo da média em $\hat{\rho}$. Matematicamente [Leonhardt 1997],

$$
\begin{aligned}
\left\langle\left\{\hat{\mathcal{Z}}_{i}, \hat{\mathcal{Z}}_{j}\right\} / 2\right\rangle_{\hat{\rho}} & =\operatorname{Tr}\left\{\hat{\rho}\left(\hat{\mathcal{Z}}_{i} \hat{\mathcal{Z}}_{j}+\hat{\mathcal{Z}}_{j} \hat{\mathcal{Z}}_{i}\right) / 2\right\} \\
& =\int d \mathcal{Z}^{2 N} W(\mathcal{Z}) \mathcal{Z}_{i} \mathcal{Z}_{j} \\
& \equiv\left\langle\mathcal{Z}_{i} \mathcal{Z}_{j}\right\rangle_{W}=V_{i j}
\end{aligned}
$$

Note que na equação (2.3.3), a média dos operadores é tomada no estado do campo descrito em termos do operador densidade $\hat{\rho}$, enquanto que na equação (2.3.5) temos a média de grandezas complexas associadas a estes mesmos operadores no espaço de fase da função de Wigner. A substituição por números complexos se deve à troca dos operadores $\hat{a}\left(\hat{a}^{\dagger}\right)$ por amplitudes complexas $\alpha\left(\alpha^{*}\right)$. Isto ocorre ao passar da descrição do estado em termos de seus operadores para a sua descrição no espaço de fase da função de Wigner [Mandel 1995]. É importante observar que operadores envolvendo produtos de ordem mais alta $\hat{q}^{m} \hat{p}^{n}$ devem ser colocados em uma forma simetrizada para que correspondam a operadores hermitianos. Isso significa somar todos os produtos possíveis de $\hat{q}$ e $\hat{p}$. Como exemplo, o operador $\left(\hat{q}^{2} \hat{p}+\hat{q} \hat{p} \hat{q}+\hat{p} \hat{q}^{2}\right) / 3$ é uma simetrização possível do operador $\hat{q}^{2} \hat{p}$.

Em relação à matriz de covariâncias, os elementos diagonais de $\mathbf{V}$ representam as variâncias das quadraturas dos modos representadas por $\Delta^{2} \hat{p}_{\omega} \equiv\left\langle\hat{p}_{\omega} \hat{p}_{\omega}\right\rangle_{W}$, por exemplo. Os elementos fora da diagonal são correlações entre diferentes operadores de quadratura, tal como $C\left(\hat{p}_{\omega} \hat{p}_{\omega^{\prime}}^{\prime}\right) \equiv\left\langle\hat{p}_{\omega} \hat{p}_{\omega^{\prime}}^{\prime}\right\rangle_{W}$. Aqui, deixamos explícito o fato de as médias serem tomadas no espaço de fase da função de Wigner, algo observado na notação \langle\rangle$_{W}$, assunto que será tratado na subseção seguinte.

Para um sistema composto por dois modos distintos do campo a matriz de covariâncias tem sua forma explícita

$$
\mathbf{V}=\left(\begin{array}{cccc}
\Delta^{2} \hat{p}_{\omega} & C\left(\hat{p}_{\omega} \hat{q}_{\omega}\right) & C\left(\hat{p}_{\omega} \hat{p}_{\omega^{\prime}}^{\prime}\right) & C\left(\hat{p}_{\omega} \hat{q}_{\omega^{\prime}}^{\prime}\right) \\
& \Delta^{2} \hat{q}_{\omega} & C\left(\hat{p}_{\omega} \hat{p}_{\omega^{\prime}}^{\prime}\right) & C\left(\hat{p}_{\omega} \hat{p}_{\omega^{\prime}}^{\prime}\right) \\
& & \Delta^{2} \hat{p}_{\omega^{\prime}}^{\prime} & C\left(\hat{p}_{\omega} \hat{p}_{\omega^{\prime}}^{\prime}\right) \\
& & \Delta^{2} \hat{q}_{\omega^{\prime}}^{\prime}
\end{array}\right),
$$


onde foram omitidos os termos que se repetem, facilmente identificados pela propriedade de simetria $\mathbf{V}=\mathbf{V}^{\mathcal{T}}$.

Estados gaussianos são completamente descritos pelos momentos de primeira e segunda ordem, sendo estes últimos organizados em uma matriz de covariâncias da forma descrita anteriormente. Conforme detalhado na referência [Simon 2000], a função de Wigner para um estado gaussiano tem sua expressão dada por

$$
W(\mathcal{Z})=\frac{1}{\pi^{N} \sqrt{\operatorname{det}(V)}} \exp \left(\frac{1}{2} \mathcal{Z} V \mathcal{Z}^{\mathcal{T}}\right)
$$

escrita para um sistema mais geral constituído por $N$ subsistemas. Assim sendo, a matriz de covariâncias carrega em si todas as informações necessárias para descrever estados gaussianos, dado que os momentos de primeira ordem, valores médios dos estados, podem ser facilmente ajustados por deslocamentos no espaço de fase. Dizemos então que para um sistema de $N$ modos, $N(2 N+1)$ momentos de segunda ordem são requeridos para descrever completamente um estado gaussiano. É neste contexto que estão inseridos os estados produzidos e analisados experimentalmente nesta tese. Neste ponto, estamos em condição de extrair informações importantes da expressão (2.3.2). Essas informações podem esclarecer o real significado dos operadores não-hermitianos $\hat{p}(\Omega)$ e $\hat{q}(\Omega)$ apresentados na equação (2.2.16).

\subsubsection{Relação entre as médias em $\hat{\rho}$ e $W$}

Definida a matriz de covariâncias, vemos que a descrição dos operadores no domínio da frequência levará a médias do tipo $\left\langle\hat{p}(\Omega) \hat{p}\left(\Omega^{\prime}\right)\right\rangle$, $\left\langle\hat{q}(\Omega) \hat{q}\left(\Omega^{\prime}\right)\right\rangle$ e $\left\langle\hat{p}(\Omega) \hat{q}\left(\Omega^{\prime}\right)\right\rangle$, difíceis de serem interpretadas intuitivamente no espaço de fase da função de Wigner $(W)$. Contrariamente, no domínio temporal, as médias de operadores tomadas no estado de um sistema descrito pelo operador densidade $\hat{\rho}$ levam à relação

$$
\operatorname{Tr}\{\hat{p}(t) \hat{\rho}\}=\int d \alpha^{2}\left[\alpha(t)+\alpha^{*}(t)\right] W\left(\alpha(t), \alpha^{*}(t)\right),
$$

de onde se vê claramente que a média tomada no operador densidade do operador de quadratura $\hat{p}(t)=\hat{a}(t)+\hat{a}^{\dagger}(t)$, correspondente a um modo bem definido do campo, é igual a média da função $p(t)=\alpha(t)+\alpha^{*}(t)$ no espaço de fase $W\left(\alpha(t), \alpha^{*}(t)\right)$. Desta forma, ficam claras as grandezas envolvidas nos cálculos das médias nos dois formalismos.

De modo sucinto, a equação anterior se escreve

$$
\langle\hat{p}(t)\rangle_{\hat{\rho}}=\left\langle\alpha(t)+\alpha^{*}(t)\right\rangle_{W}
$$

Em um contexto geral, a função de Wigner é definida tal que valha a relação

$$
\left\langle F_{S}\left(\hat{a}, \hat{a}^{\dagger}\right)\right\rangle_{\hat{\rho}}=\left\langle F\left(\alpha, \alpha^{*}\right)\right\rangle_{W}
$$


onde $F_{S}\left(\hat{a}, \hat{a}^{\dagger}\right)$ é uma função dos operadores de criação e aniquilação em ordem simétrica e $F\left(\alpha, \alpha^{*}\right)$ é a mesma função em termos dos argumentos complexos $\alpha$ e $\alpha^{*}$ da função de Wigner.

Para descobrir o que de fato ocorre com as médias de produtos dos operadores definidos em (2.2.16), consideremos a média $\left\langle p(\Omega) p\left(\Omega^{\prime}\right)\right\rangle_{W}$. Iniciamos escrevendo-a em termos de seus operadores no domínio temporal,

$$
\begin{aligned}
\left\langle p(\Omega) p\left(\Omega^{\prime}\right)\right\rangle_{W} & =\int d t e^{i \Omega t} \int d t^{\prime} e^{i \Omega t^{\prime}}\left\langle p(t) p\left(t^{\prime}\right)\right\rangle_{W} \\
& =\int d t e^{i \Omega t} \int d t^{\prime} e^{i \Omega t^{\prime}}\left\langle\hat{p}(t) \hat{p}\left(t^{\prime}\right)\right\rangle_{\hat{\rho}}
\end{aligned}
$$

em que foi utilizada a correspondência formal (2.3.10), na troca da média das grandezas complexas em $W$ pela média dos operadores em $\hat{\rho}$. Neste ponto, devemos reescrever o operador $\hat{p}(t)$ em termos dos modos do campo de frequência $\omega$. Seguindo os passos que levaram da equação (2.2.11) para (2.2.13), o operador de quadratura se escreve

$$
\begin{aligned}
\hat{p}(t) & =\hat{a}(t)+\hat{a}^{\dagger}(t) \approx \int d \Omega e^{-i \Omega t} \hat{a}_{\Omega}+\int d \Omega e^{i \Omega t} \hat{a}_{\Omega}^{\dagger} \\
& \approx \int d \Omega e^{-i \Omega t}\left(\hat{a}_{\Omega}+\hat{a}_{-\Omega}^{\dagger}\right),
\end{aligned}
$$

onde a passagem da equação (2.3.13) para a equação (2.3.14) é feita pela mudança de variável $\Omega \rightarrow-\Omega$ na integral correspondente ao operador $\hat{a}^{\dagger}(t)$.

Utilizando a equação (2.3.14), escrevemos a média $\left\langle\hat{p}(t) \hat{p}\left(t^{\prime}\right)\right\rangle_{\hat{\rho}}$ como

$$
\left\langle\hat{p}(t) \hat{p}\left(t^{\prime}\right)\right\rangle_{\hat{\rho}}=\int d \Omega \int d \Omega^{\prime} e^{-i \Omega t} e^{-i \Omega^{\prime} t}\left\langle\left(\hat{a}_{\Omega}+\hat{a}_{-\Omega}^{\dagger}\right)\left(\hat{a}_{\Omega^{\prime}}+\hat{a}_{-\Omega^{\prime}}^{\dagger}\right)\right\rangle_{\hat{\rho}}
$$

Substituindo a equação (2.3.15) em (2.3.12), obtemos o resultado

$$
\left\langle\hat{p}(\Omega) \hat{p}\left(\Omega^{\prime}\right)\right\rangle_{W}=\left\langle\left(\hat{a}_{\Omega}+\hat{a}_{-\Omega}^{\dagger}\right)\left(\hat{a}_{\Omega^{\prime}}+\hat{a}_{-\Omega^{\prime}}^{\dagger}\right)\right\rangle_{\hat{\rho}}
$$

Por fim, como sabemos que a quantidade $p(\Omega)$ é definida a partir da transformada de Fourier de $p(t)=\alpha(t)+\alpha^{*}(t)$

$$
p(\Omega)=\int d t e^{i \Omega t} p(t)=\int d t e^{i \Omega t}\left[\alpha(t)+\alpha^{*}(t)\right]=\alpha(\Omega)+\alpha^{*}(-\Omega),
$$

podemos reescrever a relação (2.3.16) como

$$
\left\langle\left[\alpha(\Omega)+\alpha^{*}(-\Omega)\right]\left[\alpha\left(\Omega^{\prime}\right)+\alpha^{*}\left(-\Omega^{\prime}\right)\right]\right\rangle_{W}=\left\langle\left(\hat{a}_{\Omega}+\hat{a}_{-\Omega}^{\dagger}\right)\left(\hat{a}_{\Omega^{\prime}}+\hat{a}_{-\Omega^{\prime}}^{\dagger}\right)\right\rangle_{\hat{\rho}} .
$$

Esse desenvolvimento mostra que a relação (2.3.10) pode ser escrita diretamente em termos das grandezas complexas $\alpha(\Omega)$ e similares, uma vez que estes aparecem de modo natural na função de Wigner. Portanto, vemos que a correspondência formal estabelecida é

$$
\left\langle F\left(\alpha(\Omega), \alpha(-\Omega), \alpha^{*}(\Omega), \alpha^{*}(-\Omega)\right)\right\rangle_{W}=\left\langle F_{S}\left(\hat{a}_{\Omega}, \hat{a}_{\Omega}^{\dagger}, \hat{a}_{-\Omega}, \hat{a}_{-\Omega}^{\dagger}\right)\right\rangle_{\hat{\rho}},
$$


em que dois modos legítimos do campo aparecem necessariamente. Isto posto, podemos dizer que os operadores $\hat{p}(\Omega)$ e $\hat{q}(\Omega)$, são na verdade uma combinação dos modos das bandas laterais $\pm \Omega$.

Resumimos estes resultados em uma tabela contendo as relações de correspondências entre os operadores de criação e aniquilação de modos do campo e as grandezas complexas existentes na representação de Wigner:

\begin{tabular}{|l|l|}
\hline \multicolumn{2}{|c|}{ Tabela de correspondências } \\
\hline Estado & $\hat{\rho} \Longleftrightarrow W\left(\alpha(t), \alpha^{*}(t)\right)$ \\
\hline Modo temporal & $\hat{a}(t) \Longleftrightarrow \alpha(t)$ \\
& $\hat{a}^{\dagger}(t) \Longleftrightarrow \alpha^{*}(t)$ \\
\hline Modo $\Omega$ & $\hat{a}_{\Omega} \Longleftrightarrow \alpha(\Omega)$ \\
& $\hat{a}_{\Omega}^{\dagger} \Longleftrightarrow \alpha^{*}(\Omega)$ \\
\hline Modo $-\Omega$ & $\hat{a}_{-\Omega} \Longleftrightarrow \alpha(-\Omega)$ \\
& $\hat{a}_{-\Omega}^{\dagger} \Longleftrightarrow \alpha^{*}(-\Omega)$ \\
\hline
\end{tabular}

Tabela 2.1: Correspondências entre operadores do campo e números complexos no espaço da função de Wigner.

Definimos nesta seção a notação para a matriz de covariâncias e as relações de correspondências entre os formalismos nos cálculos da média de operadores na descrição do operador densidade e da função de Wigner. A seguir, descreveremos o processo de fotodetecção do campo utilizando a técnica de detecção homodina. Veremos que a descrição seguinte possibilitará chegar às mesmas conclusões desta subseção.

\subsection{Fotodetecção de quadraturas do campo}

Nesta análise de fotodetecção, lidaremos com campos escalares que possuem polarização e direção de propagação bem definidas, se propagando livremente até incidir em uma região ativa do detector de área $A$, situada em um plano fixo, perpendicular à direção de propagação do campo. Primeiramente, iremos identificar as etapas envolvidas no processo de medida e, em seguida, veremos como acessar as informações sobre as quadraturas do campo através da detecção homodina. Finalmente, identificaremos o que de fato é medido e sua relação com operadores legítimos do campo. 


\subsubsection{O operador de fotodetecção}

Com as suposições anteriores e tomando o limite contínuo para um volume de quantização infinito, o campo elétrico dado na equação (2.2.7) tem sua expressão reescrita como

$$
\hat{E}(t)^{(+)}=\int_{0}^{\infty} d \omega e^{-i \omega t} \hat{a}_{\omega}
$$

em que foram omitidos os fatores multiplicativos.

A relação de comutação entre os operadores de criação e aniquilação de modos do campo passa a ser $\left[\hat{a}_{\omega}, \hat{a}_{\omega^{\prime}}^{\dagger}\right]=\delta\left(\omega-\omega^{\prime}\right)$. Vemos que o campo é uma soma de modos rotulados pela frequência $\omega$, fixada pela dependência espacial $\omega=c|\vec{k}|$. Esta frequência não possui qualquer relação com a decomposição de Fourier com a qual obtivemos as equações (2.2.16), trata-se de uma frequência de evolução livre do campo.

Como o processo de medição do campo se dá por meio de efeito fotoelétrico, é natural que o operador associado à medida de fotodeteç̧ão esteja diretamente relacionado com o operador número de fótons $\hat{a}_{\omega}^{\dagger} \hat{a}_{\omega}$. De fato, de acordo com [Glauber 1963b], o operador hermitiano que representa a fotodetecção é

$$
\begin{aligned}
\hat{I}(t) & =\hat{E}^{(-)}(t) \hat{E}^{(+)}(t) \\
& =\iint d \omega d \omega^{\prime} e^{-i\left(\omega-\omega^{\prime}\right) t} \hat{a}_{\omega}^{\dagger} \hat{a}_{\omega^{\prime}} .
\end{aligned}
$$

A média desse operador no estado que chega ao detector fornecerá a fotocorrente medida.

Para que ambas as quadraturas do campo sejam acessadas, é necessário utilizar técnicas interferométricas, pois tudo que o fotodetector permite medir é fluxo de fótons. Sendo assim, as informações obtidas por medidas diretas estão imediatamente associadas com a quadratura amplitude, uma vez que esta é proporcional à intensidade do campo. A técnica mais amplamente utilizada é a detecção homodina.

\subsubsection{Quadraturas do campo e o processo de fotodetecção}

Para estudar o processo de medida vamos considerar dois campos: um intenso, denominado portadora, que se encontra em um estado coerente com frequência $\omega_{0}$ de amplitude $\beta=|\beta| e^{-i \theta}$, denotado por $\left|\beta_{\omega_{0}}\right\rangle$ cujo espaço de Hilbert é $\mathscr{H}_{p}$; e outro com estado multimodo desconhecido onde estão as quantidades de interesse, $|\psi\rangle$ no espaço de Hilbert $\mathscr{H}_{b l}$, com número médio de fótons muito menor. Nas técnicas interferométricas utilizadas no acesso às quadraturas, o campo coerente é denominado oscilador local (LO), terminologia que também utilizaremos aqui. A detecção homodina é a técnica mais difundida e será apresentada em detalhe na seção 2.6.1. Inicialmente, consideremos a medida do estado que tem sua forma geral dada pelo produto direto dos estados citados,

$$
|\Psi\rangle=\left|\beta_{\omega_{0}}\right\rangle \otimes|\psi\rangle,
$$

cujo espaço de Hilbert no qual é definido é $\mathscr{H}=\mathscr{H}_{p} \otimes \mathscr{H}_{b l}$. 
O valor médio do operador que representa a fotodetecção (equação 2.4.2) fornece a fotocorrente $I(t)$,

$$
\begin{aligned}
I(t) & =\left\langle\Psi\left|\hat{E}^{(-)}(t) \hat{E}^{(+)}(t)\right| \Psi\right\rangle \\
& =\iint d \omega d \omega^{\prime} e^{-i\left(\omega-\omega^{\prime}\right) t}\left\langle\beta_{\omega_{0}}\left|\left\langle\psi\left|\hat{a}_{\omega}^{\dagger} \hat{a}_{\omega^{\prime}}\right| \beta_{\omega_{0}}\right\rangle\right| \psi\right\rangle .
\end{aligned}
$$

Por conta da intensidade do oscilador local, alguns termos dessa equação se destacam e são explicitados pela aplicação dos operadores de criação e aniquilação referentes à frequência $\omega^{\prime}=\omega_{0}$, pois, para um estado coerente, $\hat{a}_{\omega^{\prime}}\left|\beta_{\omega_{0}}\right\rangle=\delta\left(\omega^{\prime}-\omega_{0}\right) \beta\left|\beta_{\omega_{0}}\right\rangle$. Por exemplo, a integral envolvendo o operador $\hat{a}_{\omega^{\prime}}$ tem sua forma explícita,

$$
\int d \omega^{\prime} e^{i \omega^{\prime} t} \hat{a}_{\omega^{\prime}}\left|\beta_{\omega_{0}}\right\rangle|\psi\rangle=\left(e^{-i \omega_{0} t} \beta+\int^{\prime} d \omega^{\prime} e^{-i \omega^{\prime} t} \hat{a}_{\omega^{\prime}}\right)\left|\beta_{\omega_{0}}\right\rangle|\psi\rangle,
$$

na qual vemos duas contribuições dentro dos parênteses: a primeira correspondente ao operador $\hat{a}_{\omega_{0}}$ que atua no espaço de Hilbert $\mathscr{H}_{p}$ e a segunda, correspondente às demais frequências descritas no espaço $\mathscr{H}_{b l}$. Note a presença do apóstrofo na integral indicando que a frequência $\omega_{0}$ não é mais levada em conta neste último termo.

Realizando uma manipulação algébrica simples com a exponencial $e^{-i \omega_{0} t}$, obtemos

$$
\int d \omega^{\prime} e^{i \omega^{\prime} t} \hat{a}_{\omega^{\prime}}\left|\beta_{\omega_{0}}\right\rangle|\psi\rangle=e^{-i \omega_{0} t}(\beta+\underbrace{\int^{\prime} d \omega^{\prime} e^{-i\left(\omega^{\prime}-\omega_{0}\right) t} \hat{a}_{\omega^{\prime}}}_{\hat{a}(t)})\left|\beta_{\omega_{0}}\right\rangle|\psi\rangle .
$$

O termo entre parênteses indica que o tratamento heurístico adotado nas equações (2.1.13) e (2.2.15) aparece naturalmente neste desenvolvimento formal, pois é possível reconhecer a soma de um valor médio com um termo que faz as vezes da flutuação, formado por contribuições de frequências diferentes da frequência central $\omega_{0}$, fato que indicamos com um apóstrofo na integral.

A expressão para $I(t)$ pode ser obtida calculando de modo similar a outra integral da expressão (2.4.5) que é simplesmente o complexo conjugado da equação acima. Dito isto, podemos examinar as quantidades envolvidas em $I(t)=\| \hat{E}^{(+)}(t)|\Psi\rangle \|^{2}$, explicitamente escrita como

$$
\begin{aligned}
I(t) & \approx|\beta|^{2}+\beta^{*} \int^{\prime} d \omega^{\prime} e^{-i\left(\omega^{\prime}-\omega_{0}\right) t}\left\langle\psi\left|\hat{a}_{\omega^{\prime}}\right| \psi\right\rangle+\beta \int^{\prime} d \omega e^{i\left(\omega^{\prime}-\omega_{0}\right) t}\left\langle\psi\left|\hat{a}_{\omega}^{\dagger}\right| \psi\right\rangle \\
& \approx|\beta|^{2}+|\beta|\left\langle\psi\left|(e^{-i \theta} \underbrace{\int^{\prime} d \omega^{\prime} e^{-i\left(\omega^{\prime}-\omega_{0}\right) t} \hat{a}_{\omega^{\prime}}}_{\hat{a}(t)}+e^{i \theta} \underbrace{\int^{\prime} d \omega^{\prime} e^{i\left(\omega^{\prime}-\omega_{0}\right) t} \hat{a}_{\omega^{\prime}}^{\dagger}}_{\hat{a}^{\dagger}(t)})\right| \psi\right\rangle .
\end{aligned}
$$

Os operadores de criação e aniquilação no domínio temporal definidos aqui são os operadores lentamente variáveis apresentados na equação (2.2.12), porém manteremos a notação acima. O primeiro termo, $|\beta|^{2}$, é a intensidade média do campo, denotada nos 
experimentos como sendo a componente DC. As outras contribuições são frutos do produto entre os modos $\omega \neq \omega_{0}$ e a frequência central $\omega_{0}$ do oscilador local. Note que, mesmo sendo de intensidades muito pequenas, estes termos são amplificados pela amplitude do oscilador local. A exemplo do que foi feito nas expressões (2.1.13) e (2.2.15), os termos de intensidade cruzada entre modos de poucos fótons foram desprezados por terem contribuições muito menores para a fotocorrente quando comparados com os demais.

Podemos reconhecer a grandeza entre os parênteses da equação anterior como sendo uma quadratura generalizada temporal do campo, traduzida como amplitude ou fase dependendo da fase $\theta$ do oscilador local. Sendo assim, a equação pode ser escrita de uma forma mais compacta,

$$
I(t)=|\beta|^{2}+|\beta|\left\langle\delta \hat{I}_{\theta}(t)\right\rangle
$$

onde

$$
\delta \hat{I}_{\theta}(t)=\hat{a}(t) e^{-i \theta}+\hat{a}^{\dagger}(t) e^{i \theta}
$$

representa a flutuação da fotocorrente no tempo ${ }^{4}$.

Com o intuito de deixarmos explícita a dependência dos operadores com as frequências no entorno da frequência central $\omega_{0}$, utilizamos um tratamento similar ao realizado nas equações (2.2.11)-(2.2.13) para obter

$$
\hat{a}(t)=\int d \Omega e^{-i \Omega t} \hat{a}_{\Omega} \quad \text { e } \quad \hat{a}^{\dagger}(t)=\int d \Omega e^{i \Omega t} \hat{a}_{\Omega}^{\dagger},
$$

onde utilizamos novamente a forma compacta $\omega_{0} \pm \Omega \rightarrow \pm \Omega$ e retiramos o apóstrofo das integrais. Explicitando as frequências positivas e negativas, a quadratura generalizada passa a ser

$$
\begin{aligned}
\delta \hat{I}_{\theta}(t) & =\int d \Omega\left(e^{-i \theta} \mathrm{e}^{-i \Omega t} \hat{a}_{\Omega}+e^{i \theta} \mathrm{e}^{i \Omega t} \hat{a}_{\Omega}^{\dagger}\right) \\
& =\int_{0}^{\infty} d \Omega\left(e^{-i \theta} \mathrm{e}^{-i \Omega t} \hat{a}_{\Omega}+e^{i \theta} \mathrm{e}^{i \Omega t} \hat{a}_{\Omega}^{\dagger}+e^{-i \theta} \mathrm{e}^{i \Omega t} \hat{a}_{-\Omega}+e^{i \theta} \mathrm{e}^{-i \Omega t} \hat{a}_{-\Omega}^{\dagger}\right)
\end{aligned}
$$

Fizemos isso com o intuito de escrever a equação anterior explicitando os operadores $\hat{p}_{\Omega}$ e $\hat{q}_{\Omega}$ legítimos dos modos de frequência $\Omega$,

$$
\begin{aligned}
& \hat{p}_{\Omega}=\hat{a}_{\Omega}+\hat{a}_{\Omega}^{\dagger} \\
& \hat{q}_{\Omega}=-i\left(\hat{a}_{\Omega}-\hat{a}_{\Omega}^{\dagger}\right),
\end{aligned}
$$

\footnotetext{
${ }^{4}$ Como veremos, na técnica de detecção homodina, a equação (2.4.11) surge naturalmente como um operador que representa a subtração das fotocorrentes dos modos oriundos de uma detecção balanceada, onde a fase $\theta$ é interpretada fisicamente, e de modo preciso, como uma fase relativa entre o feixe de interesse e o oscilador local. Na descrição dada até aqui, esta fase aparece como uma propriedade que não podemos acessar diretamente sem o uso de técnicas interferométricas.
} 
com relações de comutação $\left[\hat{p}_{\Omega}, \hat{q}_{\Omega^{\prime}}\right]=2 i \delta\left(\Omega-\Omega^{\prime}\right)$, o que resulta em

$$
\begin{aligned}
\delta \hat{I}_{\theta}(t) & =\cos \theta \int_{0}^{\infty} d \Omega\left[\left(\hat{p}_{\Omega}+\hat{p}_{-\Omega}\right) \cos (\Omega t)+\left(\hat{q}_{\Omega}-\hat{q}_{-\Omega}\right) \operatorname{sen}(\Omega t)\right]+ \\
& +\operatorname{sen} \theta \int_{0}^{\infty} d \Omega\left[\left(-\hat{p}_{\Omega}+\hat{p}_{-\Omega}\right) \operatorname{sen}(\Omega t)+\left(\hat{q}_{\Omega}+\hat{q}_{-\Omega}\right) \cos (\Omega t)\right] .
\end{aligned}
$$

A flutuação da fotocorrente dada pela equação acima mostra que estamos lidando com um sistema composto pelos modos $\pm \Omega$, dado que os operadores de quadratura $\hat{p}_{ \pm \Omega}$ e $\hat{q}_{ \pm \Omega}$ aparecem explicitamente. Declarando $w \rightarrow \Omega$ e $w^{\prime} \rightarrow-\Omega$ na equação (2.3.6), podemos descrever este sistema através de uma matriz de covariâncias que carrega informações sobre as amplitudes de ruído de suas quadraturas $\left(\Delta^{2} \hat{p}_{ \pm \Omega}\right.$ e $\left.\Delta^{2} \hat{q}_{ \pm \Omega}\right)$ e correlações entre as mesmas (i.e., $C\left(\hat{p}_{\Omega} \hat{p}_{-\Omega}\right)$ e $\left.C\left(\hat{p}_{\Omega} \hat{q}_{ \pm \Omega}\right)\right)$.

Apresentamos aqui a estrutura da fotocorrente no processo de homodinagem. O passo seguinte é ver como a decomposição em Fourier influencia essa estrutura e seus efeitos sobre os modos das bandas laterais.

\subsubsection{Quadraturas segundo análise Fourier}

As informações sobre as flutuações e sua natureza estatística estão presentes nas bandas laterais do campo e são fisicamente acessíveis através da decomposição por análise de Fourier da fotocorrente medida. O procedimento geral consiste em misturar a fotocorrente $I(t)$ de entrada com um sinal periódico $I_{0}(t)$ conhecido como oscilador local eletrônico (eLO), de frequência $\Omega_{0}$ fixada pelo observador. Em seguida, o sinal $I(t) I_{0}(t)$, proveniente do misturador, atravessa um filtro passa-banda que determina a resolução em frequência que poderá ser observada. O resultado deste processo é integrado usando um filtro passa-baixa que determina o intervalo de tempo no qual será feita a média do sinal de entrada, geralmente muito maior que o período do eLO. Tipicamente, a variação das propriedades do campo ocorre em intervalos de tempo muito curtos quando comparados ao tempo de integração associado à detecção, o que permite colocar limites infinitos nas integrais. Queremos dizer que a eletrônica fornece o sinal

$$
\begin{aligned}
\int d t I(t) I_{0}(t) & =\int d t I(t) e^{i \Omega_{0} t} \\
& =I_{\Omega_{0}}
\end{aligned}
$$

onde escrevemos o sinal do eLO como $I_{0}(t)=I_{0} e^{i \Omega_{0} t}$ e omitimos o fator $I_{0}$ na equação acima.

Desprezando o valor médio, podemos usar a forma compacta (2.4.10) para a flutuação da fotocorrente a fim de escrever

$$
I_{\Omega_{0}}=\int d t\left\langle\delta \hat{I}_{\theta}(t)\right\rangle e^{i \Omega_{0} t}
$$


Substituindo a expressão (2.4.13) obtida para $\delta \hat{I}_{\theta}$ e invertendo a ordem das integrais temos

$$
\begin{array}{r}
I_{\Omega_{0}}=\left\langle\int d \Omega \int d t e^{i \Omega_{0}}\left(e^{-i \theta} e^{-i \Omega t} \hat{a}_{\Omega}+e^{i \theta} e^{i \Omega t} \hat{a}_{\Omega}^{\dagger}\right)\right\rangle \\
=\left\langle e^{-i \theta} \hat{a}_{\Omega_{0}}+e^{i \theta} \hat{a}_{-\Omega_{0}}^{\dagger}\right\rangle
\end{array}
$$

Assim, no fim da análise de Fourier mostramos que a componente espectral da fotocorrente correspondente ao sinal efetivamente medido é uma grandeza complexa associada ao operador não-hermitiano

$$
\hat{I}_{\Omega}=e^{-i \theta} \hat{a}_{\Omega}+e^{i \theta} \hat{a}_{-\Omega}^{\dagger},
$$

com frequência $\Omega$ definida pelo eLO. Vê-se claramente que $\hat{I}_{\Omega}^{\dagger}=\hat{I}_{-\Omega}$. Podemos reescrever a equação precedente explicitando os operadores hermitianos das quadraturas dos campos nos modos $\Omega$ e $-\Omega$. Fazemos isso definindo os operadores das combinações simétricas (s) e antissimétricas (a):

$$
\hat{p}_{s(a)}=\left(\hat{p}_{\Omega} \pm \hat{p}_{-\Omega}\right) / \sqrt{2} \text { e } \hat{q}_{s(a)}=\left(\hat{q}_{\Omega} \pm \hat{q}_{-\Omega}\right) / \sqrt{2},
$$

em que $s$ e a representam, respectivamente, a combinação dada pela soma e subtração dos operadores $\hat{p}_{ \pm \Omega}$ e $\hat{q}_{ \pm \Omega}$.

Definindo também as correspondentes quadraturas generalizadas $\hat{X}_{s}^{\theta}$ e $\hat{X}_{a}^{\theta+\frac{\pi}{2}}$, podemos verificar que os termos em quadratura da fotocorrente $\hat{I}_{\Omega}=\sqrt{2}\left(\hat{I}_{\cos }+i \hat{I}_{\text {sen }}\right)$, são dados por

$$
\begin{aligned}
\hat{I}_{\mathrm{cos}} & =\cos \theta \hat{p}_{s}+\operatorname{sen} \theta \hat{q}_{s} \equiv \hat{X}_{s}^{\theta} \\
\hat{I}_{\mathrm{sen}} & =\cos \theta \hat{q}_{a}-\operatorname{sen} \theta \hat{p}_{a} \equiv \hat{X}_{a}^{\theta+\frac{\pi}{2}}
\end{aligned}
$$

Assim, em princípio, a medida das componentes $\hat{I}_{\text {cos }}$ e $\hat{I}_{\text {sen }}$ da fotocorrente obtidas pela decomposição de Fourier pode trazer de maneira direta informações sobre as combinações simétricas e antissimétricas dos modos das bandas. Podemos então dizer que cada um dos sinais em quadratura é uma medida de um modo único do campo, ambos acessíveis em virtude da relação de comutação $\left[\hat{I}_{\cos }, \hat{I}_{\text {sen }}\right]=0$.

Esses dois sinais podem ser medidos de forma independente através de um procedimento relativamente simples, misturando a fotocorrente com dois sinais em quadratura, conforme ilustrado na figura abaixo.

A exemplo do que foi feito para os modos específicos das bandas laterais, podemos escrever a matriz de covariâncias (2.3.6) na base $\left\{\hat{p}_{s}, \hat{q}_{s}, \hat{p}_{a}, \hat{q}_{a}\right\}$, declarando $w \rightarrow s$ e $w^{\prime} \rightarrow a$, obtendo as amplitudes de ruído dessas novas quadraturas, $\Delta^{2} \hat{p}_{s(a)}$ e $\Delta^{2} \hat{q}_{s(a)}$, e correlações entre as mesmas [i.e, $C\left(\hat{p}_{s} \hat{p}_{a}\right)$ e $\left.C\left(\hat{p}_{s} \hat{q}_{s(a)}\right)\right]$.

Para determinar a contribuição desses modos para a potência de ruído em uma determinada frequência segundo a análise Fourier, vejamos como é calculado o espectro de ruído. 


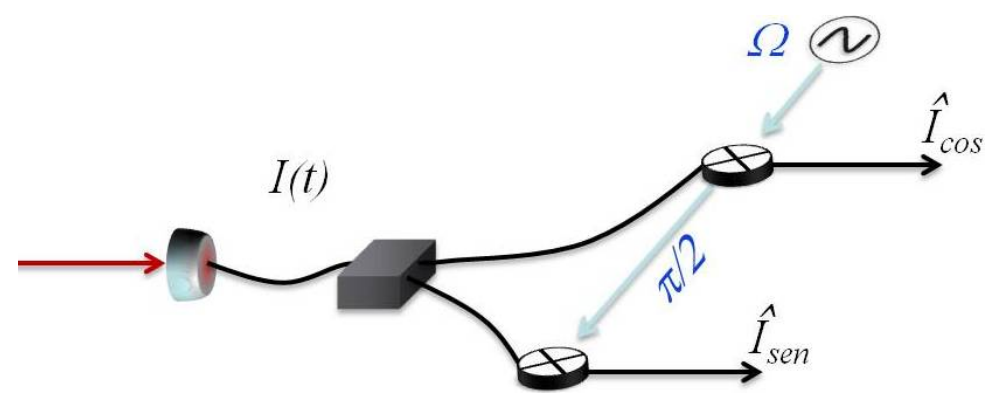

Figura 2.2: Esquema de medida das fotocorrentes em quadratura.

\subsubsection{Espectro de ruído}

Após adquirir os sinais das fotocorrentes, utilizamos o Teorema de Wiener-Kintchine para obter a potência de ruído $S(\Omega)$ associado à fotocorrente $\hat{I}(t)$ do campo. Faz-se isso, calculando a transformada de Fourier da função de autocorrelação temporal [Mandel 1995]

$$
C\left(t, t^{\prime}\right)=\langle\delta \hat{I}(t) \delta \hat{I}(t+\tau)\rangle, \quad \tau=t-t^{\prime}
$$

que determina como o valor da flutuação da fotocorrente no tempo $t$ se relaciona com o valor no instante de tempo $t^{\prime}$. Para processos estacionários, definidos aqui como aqueles onde $C\left(t, t^{\prime}\right)$ depende somente da diferença de tempo $\tau=t-t^{\prime}$, o Teorema de WienerKintchine [Mandel 1995] afirma que $S(\Omega)$ é dado por

$$
S(\Omega)=\int e^{i \Omega \tau}\langle\delta \hat{I}(t) \delta \hat{I}(t+\tau)\rangle d \tau
$$

ou, após substituir os operadores dependentes do tempo pelas suas transformadas de Fourier,

$$
2 \pi S(\Omega) \delta\left(\Omega-\Omega^{\prime}\right)=\left\langle\hat{I}_{\Omega} \hat{I}_{-\Omega^{\prime}}\right\rangle
$$

reescrita como

$$
\left\{\begin{array}{l}
S(\Omega)=\left\langle\hat{I}_{\Omega} \hat{I}_{-\Omega}\right\rangle, \\
\left\langle\hat{I}_{\Omega} \hat{I}_{\Omega^{\prime}}\right\rangle=0, \text { para } \Omega^{\prime} \neq-\Omega .
\end{array}\right.
$$

Nas linhas seguintes, quando nos referirmos a estados estacionários, estamos nos reportando a estados que geram fotocorrentes que satisfazem a equação acima. A função delta surge por causa do limite de integração infinito na equação (2.2.13). No entanto, essa divergência é contornada pelo fato de medidas serem realizadas em um tempo finito, além de serem utilizados filtros de frequência com uma dada largura de banda o que define a frequência $\Omega$ dentro de um certo intervalo. Desse modo não medimos exatamente o espectro de ruído e sim algo proporcional a ele.

Utilizando as expressões das quadraturas da fotocorrente medida, dadas pelas equações (2.4.25) e (2.4.26), vemos que o espectro de ruído dado pela equação (2.4.30) se escreve como

$$
S(\Omega)=\frac{1}{2}\left\langle\hat{I}_{\text {cos }}^{2}\right\rangle+\frac{1}{2}\left\langle\hat{I}_{\text {sen }}^{2}\right\rangle \equiv \frac{1}{2} \Delta^{2} \hat{X}_{s}^{\theta}+\frac{1}{2} \Delta^{2} \hat{X}_{a}^{\theta+\frac{\pi}{2}}
$$


Esta expressão nos mostra que a amplitude do ruído medido tem contribuições de dois operadores de quadratura de modos distintos, que correspondem respectivamente às combinações simétrica e antissimétrica de frequências $\Omega$ e $-\Omega$. Logo, a amplitude de ruído não traz informações sobre um modo legítimo do campo, mas sim de uma mistura estatística de modos. No entanto, sob certas circunstâncias, apresentadas nas seções seguintes sobre as técnicas de detecção homodina e auto-homodina, as medidas de uma única quadratura eletrônica $\left(\hat{I}_{\text {cos }}\right.$ ou $\left.\hat{I}_{\text {sen }}\right)$ podem representar de fato um modo único do campo.

Quando levamos em conta a estacionariedade das flutuações da fotocorrente dada pela equação (2.4.30), o cálculo de $\left\langle\hat{I}_{\Omega} \hat{I}_{\Omega}\right\rangle$, utilizando a equação (2.4.23) e tendo em mente as relações $(2.4 .25)$ e (2.4.26), nos mostra que

$$
\left\langle\hat{I}_{\Omega} \hat{I}_{\Omega}\right\rangle=0 \Longrightarrow\left\{\begin{array}{l}
\Delta^{2} \hat{X}_{s}^{\theta}=\Delta^{2} \hat{X}_{a}^{\theta+\frac{\pi}{2}} \\
\left\langle\Delta^{2} \hat{X}_{s}^{\theta} \Delta^{2} \hat{X}_{a}^{\theta+\frac{\pi}{2}}\right\rangle=0 .
\end{array}\right.
$$

Vemos então que a estacionariedade implica perfeita simetria entre as estatísticas das componentes eletrônicas em quadratura e completa ausência de correlações entre elas.

Neste caso, podemos afirmar que a amplitude do ruído medido corresponde de fato à variância de modos legítimos do campo, sendo uma medida da combinação simétrica ou antissimétrica dos modos das bandas laterais, uma vez que são iguais. Em princípio, trata-se de um procedimento relativamente simples de ser testado, pois para isso basta realizar a medida das fotocorrentes em quadratura, conforme ilustrado na figura 2.2, comparando as flutuações de cada uma e as correlações entre elas, a fim de confirmar se estamos lidando com fotocorrentes estacionárias e dessa forma reconhecer a medida de um modo bem definido do campo.

Em uma perspectiva mais geral, podemos interpretar a potência de ruído $S(\Omega)$, de acordo com a equação (2.4.30), como um teste experimental direto do critério de emaranhamento de Duan et al. [Duan 2000] aplicado às bandas laterais. Caso seja verificada a presença de compressão de ruído, temos, além dessa característica quântica, a presença de emaranhamento entre os modos $\Omega$ e $-\Omega$. Exporemos este critério no próximo capítulo.

Estados com compressão de ruído são assim definidos por apresentarem em suas quadraturas flutuações abaixo de um nível de ruído bem estabelecido conhecido como shotnoise ou ruído quântico padrão. Este nível de ruído é uma das principais características de outra classe de estados de mínima incerteza, os estados coerentes. Nas próximas seções apresentaremos a definição do shot-noise e tentaremos mostrar em uma análise simples sua relação com o caráter corpuscular da luz.

As relações (2.4.30) podem não ser resultado somente da estacionariedade, sendo necessários outros ingredientes para ganhar mais informações sobre o estado quântico estudado. $\mathrm{Na}$ análise feita acima, não consideramos em nenhum momento o efeito da difusão da fase relativa entre o oscilador ótico e eletrônico, algo que pode ter grande importância para a medida das fotocorrentes em quadratura. Trataremos deste assunto a seguir. 


\subsubsection{O efeito da mistura de fase}

Mesmo que as componentes da fotocorrente sejam em princípio mensuráveis, é necessário garantir que exista um travamento da fase relativa entre o eLO e o oscilador local ótico (LO) de modo a tornar possível a escolha de uma das quadraturas $\left(\hat{I}_{\cos }\right.$ ou $\left.\hat{I}_{\text {sen }}\right)$ [Collett 1987]. Contudo, na prática, o oscilador local ótico sofre difusão de fase em tempos relativamente curtos [Kawakubo 2010]. Caso estejamos considerando medidas em uma determinada janela de tempo suficiente para que o efeito da difusão de fase seja considerável, haverá uma variação do operador de medida entre medidas consecutivas.

Podemos analisar o efeito da variação da fase relativa escrevendo o operador de medida da fotocorrente como uma combinação linear de suas quadraturas da seguinte maneira

$$
\hat{I}_{\phi}=\cos \phi \hat{I}_{\cos }+\operatorname{sen} \phi \hat{I}_{\mathrm{sen}}
$$

onde $\phi$ representa a fase relativa entre o LO e o eLO. Consideraremos que esta fase permanece constante durante a tomada de uma única medida mas varia entre medidas consecutivas, sendo alterada por uma quantidade $\phi^{\prime}$ a cada medida. Sendo assim, a estatística de uma sequência de medidas deve levar em conta essa variação.

Isso nos diz que os momentos de ordem superior da fotocorrente medida são na verdade uma média dos momentos do operador $\hat{I}_{\phi}$ obtidos em um conjunto de medidas consecutivas. Portanto, a variância de qualquer componente da fotocorrente é uma mistura de variâncias das componentes ideais,

$$
\begin{aligned}
\Delta^{2} \hat{I}_{\phi} & =\frac{1}{2 \pi} \int d \phi^{\prime}\left\langle\hat{I}_{\phi+\phi^{\prime}} \hat{I}_{\phi+\phi^{\prime}}\right\rangle \\
& =\frac{1}{2} \Delta^{2} \hat{I}_{\cos }+\frac{1}{2} \Delta^{2} \hat{I}_{\mathrm{sen}}, \quad \forall \phi
\end{aligned}
$$

O mesmo raciocínio pode ser aplicado ao cálculo das correlações entre as componentes em quadratura da fotocorrente,

$$
\begin{aligned}
C_{\hat{I}_{\phi} \hat{I}_{\phi+\frac{\pi}{2}}} & =\frac{1}{2 \pi} \int d \phi^{\prime}\left\langle\hat{I}_{\phi+\phi^{\prime}} \hat{I}_{\phi+\frac{\pi}{2}+\phi^{\prime}}\right\rangle \\
& =\left\langle\hat{I}_{\cos } \hat{I}_{\mathrm{sen}}-\hat{I}_{\mathrm{sen}} \hat{I}_{\mathrm{cos}}\right\rangle=0, \quad \forall \phi,
\end{aligned}
$$

pois $\left[\hat{I}_{\mathrm{cos}}, \hat{I}_{\mathrm{sen}}\right]=0$, independente do estado quântico. Os resultados acima podem ser resumidos como

$$
\left\{\begin{array}{l}
\Delta^{2} \hat{I}_{\phi}=\Delta^{2} \hat{I}_{\phi+\frac{\pi}{2}} \\
C_{\hat{I}_{\phi} \hat{I}_{\phi+\frac{\pi}{2}}}=0
\end{array}\right\} \Longleftrightarrow\left\{\begin{array}{l}
\Delta^{2} \hat{X}_{s}^{\phi}=\Delta^{2} \hat{X}_{a}^{\phi+\frac{\pi}{2}} \\
\left\langle\Delta^{2} \hat{X}_{s}^{\phi} \Delta^{2} \hat{X}_{a}^{\phi+\frac{\pi}{2}}\right\rangle=0 .
\end{array}\right\}
$$

Vemos que, não havendo relação de fase bem definida entre o eOL e OL, as quadraturas da fotocorrente apresentam a mesma estatística, o que torna arbitrária a escolha da fase $\phi$. Além disso, comparando as equações (2.4.38) e (2.4.32), observamos que o efeito da difusão 
de fase nos leva às mesmas previsões de uma fotocorrente estacionária, independente do estado quântico sobre o qual são calculadas as médias.

As consequências das relações (2.4.38) são evidentes quando olhamos os vínculos impostos sobre as matrizes de covariâncias $\mathbf{V}_{-\boldsymbol{\Omega} / \boldsymbol{\Omega}} \mathrm{e} \mathbf{V}_{\mathbf{s} / \mathbf{a}}$, escritas nas bases $\left\{\hat{p}_{\Omega}, \hat{q}_{\Omega}, \hat{p}_{-\Omega}, \hat{q}_{-\Omega}\right\}$ e $\left\{\hat{p}_{s}, \hat{q}_{s}, \hat{p}_{a}, \hat{q}_{a}\right\}$, respectivamente. Para os modos simétricos e antissimétricos temos, a menos de rotações das quadraturas, as igualdades entre variâncias $\Delta^{2} \hat{p}_{s}=\Delta^{2} \hat{q}_{a} \equiv \alpha$, $\Delta^{2} \hat{q}_{s}=\Delta^{2} \hat{p}_{a} \equiv \beta$ e $C\left(\hat{p}_{s} \hat{q}_{s}\right)=-C\left(\hat{p}_{a} \hat{q}_{a}\right) \equiv \gamma$ para correlações entre quadraturas de um mesmo modo, sendo ainda possível verificar correlações entre quadraturas de modos distintos $C\left(\hat{p}_{s} \hat{p}_{a}\right)=C\left(\hat{q}_{s} \hat{q}_{a}\right)=\delta$. Dada a relação entre as bases segundo as definições dos modos $\hat{p}_{s(a)}$ e $\hat{q}_{s(a)}$, verificamos também que $\Delta^{2} \hat{p}_{-\Omega}=\Delta^{2} \hat{q}_{-\Omega} \equiv \alpha^{\prime}, \Delta^{2} \hat{p}_{\Omega}=\Delta^{2} \hat{q}_{\Omega} \equiv \beta^{\prime}$, $C\left(\hat{p}_{-\Omega} \hat{p}_{\Omega}\right)=-C\left(\hat{q}_{-\Omega} \hat{q}_{\Omega}\right)=\gamma^{\prime}$ e $C\left(\hat{p}_{-\Omega} \hat{q}_{\Omega}\right)=-C\left(\hat{q}_{-\Omega} \hat{p}_{\Omega}\right)=\delta^{\prime}$. Obtemos, então, as matrizes de covariâncias

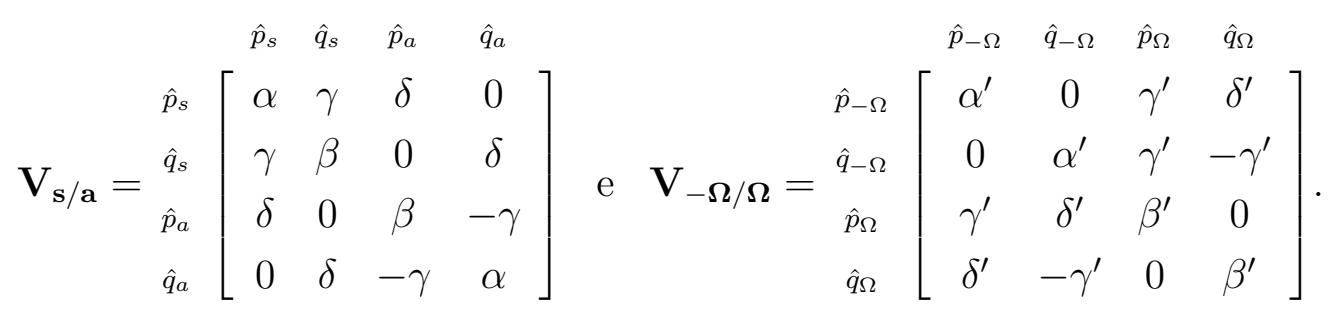

Essa descrição é geral considerando que exista a difusão de fase, independente do estado quântico. Portanto, para que o sistema seja completamente caracterizado, é necessário obter informações subjacentes a este efeito. Para uma descrição completa do estado produzido por nosso sistema, são necessárias as medidas de todos os elementos de uma das matrizes acima, uma vez que estas estão diretamente relacionadas por transformações unitárias. Analisaremos brevemente a medida desses termos, sendo este assunto tratado de modo mais detalhado na tese de outro estudante do grupo, Felippe A. S. Barbosa.

Os resultados apresentados no decorrer dessa tese são frutos de medidas realizadas com um único oscilador local eletrônico e sem travamento de fase relativa entre ele e o oscilador local ótico. Por conta disso, após algumas considerações, veremos em nosso tratamento que o estado de um campo de luz será examinado como um único modo formado pela combinação das bandas laterais, sem considerar os modos $\Omega$ e $-\Omega$ como subsistemas.

Na subseção anterior mencionamos que a existência de emaranhamento entre bandas laterais pode ser verificada se o ruído dado pela equação (2.4.30) apresentar compressão, valor abaixo do limite estabelecido pelo ruído existente em estados coerentes da luz, o shot-noise. Nas duas próximas seções trataremos deste assunto, mostrando como é feita a calibração do ruído quântico padrão e como são medidas as flutuações das quadraturas do campo relativas ao shot-noise.

\subsection{Referência de ruído}

Existem estados quânticos para os quais a relação de incerteza de Heisenberg se limita 
à igualdade e que permitem explorar a analogia entre as variáveis dinâmicas do oscilador harmônico clássico e as quadraturas aqui definidas [Stephen 1987]. Introduzido na teoria quântica por Roy J. Glauber [Glauber 1963a], estes são os chamados estados coerentes, autoestados do operador de aniquilação,

$$
\hat{a}|\alpha\rangle=\alpha|\alpha\rangle
$$

onde $\alpha$ é o autovalor de $\hat{a}$ que pode ser interpretado como uma amplitude complexa, análoga à amplitude de um oscilador harmônico. Escrito na base de Fock $\{|n\rangle\}$, onde $n$ indica um número bem definido de fótons, $|\alpha\rangle$ é dado por

$$
|\alpha\rangle=e^{-|\alpha|^{2} / 2} \sum_{n=0}^{\infty} \frac{\alpha^{n}}{\sqrt{n !}}|n\rangle .
$$

Com esta expressão, podemos calcular a probabilidade $|\langle n \mid \alpha\rangle|^{2}$ de encontrar $n$ fótons no estado $|\alpha\rangle$, obtendo como resultado uma distribuição poissoniana,

$$
\mathcal{P}(n)=\frac{1}{n !} e^{-|\alpha|^{2}}|\alpha|^{2 n}
$$

que descreve uma sucessão de eventos estatisticamente independentes, como é o caso da chegada de fótons ao fotodetector. Uma característica importante desse tipo de distribuição é a igualdade entre a variância e número médio de fótons,

$$
\Delta^{2} \hat{N}=\langle\hat{N}\rangle=|\alpha|^{2}
$$

onde $\hat{N}=\hat{a}^{\dagger} \hat{a}$ é o operador número.

Calculado para um estado coerente, o valor médio do campo elétrico monocromático dado pela equação (2.2.6) é

$$
\langle\alpha|\hat{\mathbf{E}}| \alpha\rangle=\sqrt{\frac{8 \pi \hbar \omega_{\lambda}}{L^{3}}} \vec{\epsilon}_{\lambda}|\alpha| \operatorname{sen}\left(\vec{k} \cdot \vec{r}-\omega_{\lambda} t+\varphi\right),
$$

em que $\alpha=|\alpha| e^{i \varphi}$. Vemos nessa expressão que as grandezas $|\alpha|$ e $\varphi$ representam, de fato, a amplitude e a fase do campo. A variância de $\vec{E}$ mostra um resultado bastante interessante

$$
\Delta^{2} \hat{\mathbf{E}}=\langle\alpha|\hat{\mathbf{E}} \cdot \hat{\mathbf{E}}| \alpha\rangle-\langle\alpha|\hat{\mathbf{E}}| \alpha\rangle \cdot\langle\alpha|\hat{\mathbf{E}}| \alpha\rangle=\frac{2 \pi \hbar \omega_{k}}{L^{3}} .
$$

Em um estado coerente, a amplitude das flutuações do campo elétrico calculadas permanece constante e igual às flutuações no vácuo $|0\rangle$, independente do estado $|\alpha\rangle$. A essas flutuações é dado o nome ruído quântico padrão ou shot-noise. Para nosso tratamento, é útil normalizar as flutuações do campo por este ruído de modo a obtermos sempre $\Delta^{2} \vec{E}=1$ para os estados coerentes. Isso porque existem estados para os quais as flutuações do campo têm amplitudes menores que as obtidas em estados coerentes, o que determina o caráter intrinsecamente quântico do sistema [Teich 1989]. Conhecidos como 


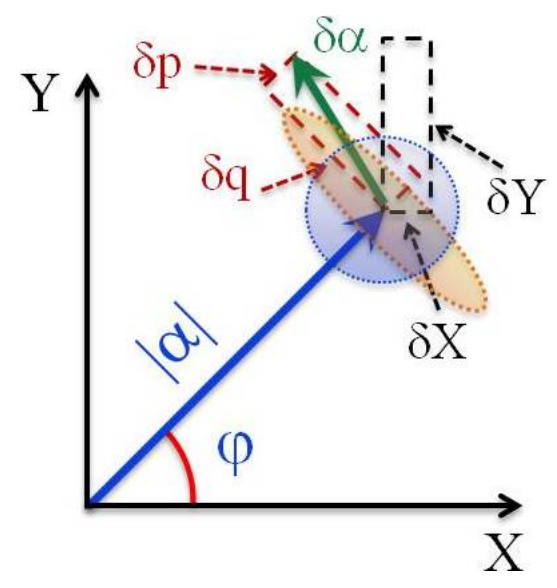

Figura 2.3: Representação do campo e suas flutuações no diagrama de Fresnel. O vetor partindo da origem representa o valor médio do campo e o círculo pontilhado o ruído de um estado coerente, shot-noise. A elipse indica compressão de ruído em uma quadratura em detrimento das flutuações na quadratura conjugada para que não haja violação da relação de incerteza de Heisenberg.

estados comprimidos, estes também pertencem à classe de estados de mínima incerteza e são de fundamental importância para ótica quântica, constituindo ainda a base de critérios de emaranhamento de estados gaussianos em variáveis contínuas. A figura mostra a representação do campo num diagrama de fasores incluindo esses novos elementos.

A seguir falaremos mais sobre o ruído quântico padrão e sua interpretação (talvez) um pouco mais intuitiva, além de mostrar como é feita sua calibração.

\subsection{O ruído quântico padrão ou shot-noise}

O ruído quântico padrão (ou shot-noise) é um dos principais conceitos deste trabalho. Trata-se de uma característica intrínseca à natureza quântica da luz e serve como referência para todos os ruídos medidos no laboratório.

A fim de ganhar intuição sobre o shot noise, podemos imaginar um campo de luz monocromática que incide sobre um divisor de feixes com transmissão e reflexão iguais. Classicamente, somos levados a pensar que as flutuações de intensidade do campo de entrada são igualmente transmitidas com metade de sua amplitude para cada campo oriundo da divisão. Sendo assim, espera-se que a amplitude das flutuações do sinal de subtração desses dois campos apresente valor nulo.

No entanto, no cenário quântico a presença de flutuações de intensidade no campo está associada às variações no fluxo de fótons. Ao chegar no divisor de feixes, cada fóton possui $50 \%$ de chance de ser transmitido ou refletido, o que torna as reflexões e transmissões aleatórias. Cada campo passa a ter de modo independente a sua própria aleatoriedade no fluxo de fótons. Portanto, a subtração das flutuações ainda dará origem a 
uma flutuação mensurável, inerente à natureza da luz. Mostramos anteriormente que, para um estado coerente, a estatística de fótons é poissoniana, um tipo de distribuição muitas vezes associada com eventos raros, com baixa probabilidade de ocorrência [Salinas 1997].

Tomando esta ideia como base, vamos considerar um problema simples apresentado na referência [Fox 2000]. Consideremos um feixe laser estável com intensidade constante de modo que não haja variação no fluxo de fótons com o tempo. Para um feixe de luz com uma potência constante, o número médio de fótons em um segmento de comprimento $L$ é dado por

$$
\bar{n}=\frac{\Phi L}{c},
$$

onde $\Phi$ é o fluxo de fótons que passam por uma seção transversal do segmento por unidade de tempo. O comprimento $L$ é considerado suficientemente grande de tal modo que o número médio de fótons seja um número inteiro bem definido.

Lança-se agora o seguinte problema: divida o segmento $L$ em $N$ partes, gerando $L / N$ subsegmentos. Faça $N$ ser muito grande, tal que a probabilidade $p=\bar{n} / N$ de encontrar um fóton dentro de um subsegmento seja muito pequena, tornando a chance de encontrar dois ou mais fótons em um dos segmentos $L / N$ desprezível. Qual a probabilidade $\mathcal{P}(n)$ de encontrar $n$ fótons dentro do segmento $L$ contendo $N$ subsegmentos?

Para encontrar a resposta, basta calcular a probabilidade de encontrar $n$ subsegmentos com 1 fóton e os $(N-n)$ restantes vazios. O resultado é uma distribuição binomial

$$
\mathcal{P}(n)=\frac{N !}{(N-n) ! n !} p^{n}(1-p)^{N-n}
$$

Substituindo $p=\bar{n} / N$ e fazendo um rearranjo simples obtemos

$$
\mathcal{P}(n)=\frac{1}{n !}\left(\frac{N !}{(N-n) ! N^{n}}\right) \bar{n}^{n}\left(1-\frac{\bar{n}}{N}\right)^{N-n} .
$$

Agora, vamos tomar o limite $N \rightarrow \infty$. Calculando o logaritmo da equação acima e utilizando a aproximação de Stirling $(\ln N ! \approx N \ln N-N)$, válida para $N$ suficientemente grande, é possível mostrar que o primeiro e o segundo termo entre parênteses na equação (2.6.3) são aproximadamente iguais a 1 e $\exp (-\bar{n})$, respectivamente. Como resultado dessas aproximações temos

$$
\mathcal{P}(n)=\frac{\bar{n}^{n}}{n !} e^{(-\bar{n})}, n=0,1,2, \ldots
$$

que descreve uma distribuição Poissoniana.

Este tipo de estatística é encontrada em processos aleatórios discretos, que fornecem como resultado números inteiros. Podemos, por exemplo, considerar o processo de contagem em um sistema que detecta fótons individuais de um feixe de luz coerente. Os valores registrados variam acima e abaixo da média e a probabilidade de registrar $n$ fótons é dada pela distribuição Poissoniana (2.6.4). No caso ideal, cada fóton que incide na superfície 
do detetor irá provocar a emissão de um elétron, dando origem a uma fotocorrente. A distribuição $\mathcal{P}(n)$ é diretamente representada pela variação dessa fotocorrente. No domínio temporal, isto significa que os fótons estão chegando ao fotodetector em tempos diferentes, de forma aleatória [Bachor 1998].

Uma distribuição Poissoniana é caracterizada unicamente pelo valor médio $\bar{n}$, uma vez que as flutuações estatísticas quantificadas pela variância, $\Delta^{2} n$, são iguais ao próprio valor médio.

Os estados coerentes descritos na seção anterior possuem essas características. Pertencem à classe de estados com mínima incerteza, distribuída igualmente entre as variáveis canonicamente conjugadas, obedecendo a igualdade da relação de incerteza de Heisenberg. São eles que definem o shot-noise. A motivação para este nome parte da análise de flutuações de corrente em circuitos elétricos, por Schottky em 1918 [Schottky 1918], devido ao caráter discreto dos portadores de carga. Schottky fez analogia entre a chegada de elétrons em ânodos e o granizo que caía sobre uma superfície gerando sons parecidos com tiros, daí o termo "shot".

A seguir apresentamos como medir o shot-noise para um feixe de luz intenso e, uma vez medido, como utilizá-lo como referência para os demais ruídos medidos.

\subsubsection{Como é medido o shot-noise?}

O limite clássico associado ao ruído das quadraturas do campo pode ser determinado a partir das flutuações de intensidade de um feixe coerente. Serão descritas aqui as técnicas empregadas em nosso experimento para a calibração do shot-noise e os métodos utilizados para torná-lo uma referência para os demais ruídos.

Considere dois campos $\vec{E}_{1}$ e $\vec{E}_{2}$ que incidem em um divisor de feixes (figura 2.4), gerando dois campos de saída $\vec{E}_{3}$ e $\vec{E}_{4}$, relacionados entre si através da equação matricial

$$
\left(\begin{array}{c}
E_{3} \\
E_{4}
\end{array}\right)=\left(\begin{array}{ll}
r & t \\
t & r
\end{array}\right)\left(\begin{array}{c}
E_{1} \\
E_{2}
\end{array}\right),
$$

em que $r$ e $t$ são os coeficientes complexos de reflexão e transmissão do divisor de feixes ilustrado na figura 2.4 .

A conservação de energia impõe $\left|\vec{E}_{1}\right|^{2}+\left|\vec{E}_{2}\right|^{2}=\left|\vec{E}_{3}\right|^{2}+\left|\vec{E}_{4}\right|^{2}$, o que implica

$$
\left\{\begin{array}{l}
|r|^{2}+|t|^{2}=1 \\
r^{*} t+r t^{*}=0
\end{array} .\right.
$$

Se escrevermos $r=|r| e^{i \varphi_{r}}$ e $t=|t| e^{i \varphi_{t}}$, é possível mostrar que a diferença de fase entre os campos refletidos e transmitidos deve ser igual a $\pi$. Utilizando esses resultados e introduzindo os operadores de aniquilação através das correspondências

$$
\begin{aligned}
& E_{1} \rightarrow \hat{a}, E_{2} \rightarrow \hat{b}, \\
& E_{3} \rightarrow \hat{c}, E_{4} \rightarrow \hat{d},
\end{aligned}
$$




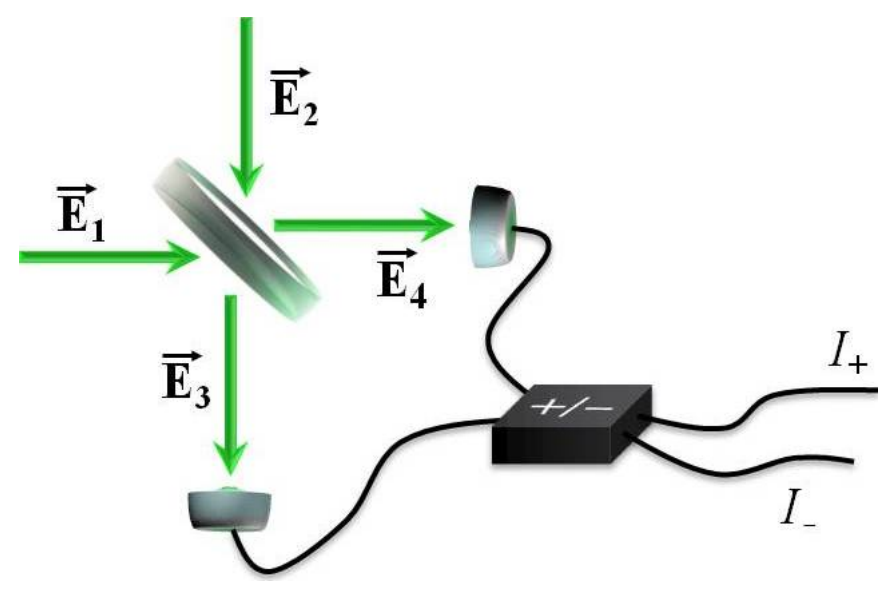

Figura 2.4: Esquema de um divisor de feixes.

obtemos

$$
\left(\begin{array}{c}
\hat{c} \\
\hat{d}
\end{array}\right)=\left(\begin{array}{cc}
|r| & -|t| \\
|t| & |r|
\end{array}\right)\left(\begin{array}{l}
\hat{a} \\
b
\end{array}\right) .
$$

Para o caso de uma detecção balanceada $(|r|=|t|=1 / \sqrt{2})$, obtemos os campos de saída

$$
\hat{c}=\frac{1}{\sqrt{2}}(\hat{a}+\hat{b}), \quad \hat{d}=\frac{1}{\sqrt{2}}(\hat{a}-\hat{b}) .
$$

No processo de deteç̧ão são usados fotodetectores sensíveis aos operadores número, uma vez que o efeito fotoelétrico é o responsável pela conversão de intensidade dos campos em fotocorrente. Dessa forma, os operadores hermitianos que representam a fotodetecção dos campos $\hat{c}$ e $\hat{d}$ são dados por

$$
\hat{I}_{c}=k \hat{c}^{\dagger} \hat{c} \quad \text { e } \hat{I}_{d}=k \hat{d}^{\dagger} \hat{d}
$$

onde $k$ é um fator constante (geralmente denominado ganho) introduzido pela eletrônica envolvida na detecção. Se o valor de $k$ realmente não variar com a intensidade do campo incidente, dizemos que a eletrônica possui uma resposta linear, algo fácil de ser testado experimentalmente.

Informações importantes sobre os campos de entrada podem ser obtidas através da soma e subtração das fotocorrentes medidas, escritas em termos dos campos de entrada como

$$
\begin{gathered}
I_{+}=\hat{I}_{c}+\hat{I}_{d}=k\left(\hat{a}^{\dagger} \hat{a}+\hat{b}^{\dagger} \hat{b}\right) \\
I_{-}=\hat{I}_{c}-\hat{I}_{d}=k\left(\hat{a}^{\dagger} \hat{b}+\hat{a} \hat{b}^{\dagger}\right) .
\end{gathered}
$$

Suponhamos um sistema formado por campos de entrada representados pelos estados $\left|\Phi_{a}\right\rangle$ e $|\beta\rangle$, tal que o estado geral seja $|\Psi\rangle=\left|\Phi_{a}\right\rangle \otimes|\beta\rangle$. Para simplificar, sem perder em 
generalidade, vamos considerar o estado correspondente ao campo $\hat{b}$ como sendo coerente, $\hat{b}|\beta\rangle=\beta|\beta\rangle$. Com isso, podemos calcular as variâncias dos sinais de soma e subtração,

$$
\Delta^{2} I_{( \pm)}=\left\langle I_{ \pm}^{2}\right\rangle-\left\langle I_{ \pm}\right\rangle^{2}
$$

Após algumas manipulações algébricas, obtém-se

$$
\begin{aligned}
\Delta^{2} I_{-} & =k^{2}\left(\left\langle\hat{a}^{\dagger} \hat{a}\right\rangle+|\beta|^{2}\right)+k^{2}\left[\left\langle\left(\beta \hat{a}^{\dagger}+\beta^{*} \hat{a}\right)^{2}\right\rangle-\left\langle\left(\beta\left\langle\hat{a}^{\dagger}\right\rangle+\beta^{*}\langle\hat{a}\rangle\right)^{2}\right\rangle\right], \\
\Delta^{2} I_{+} & =k^{2}\left[\left\langle: \Delta^{2} \hat{n}_{a}:\right\rangle+\left\langle\hat{a}^{\dagger} \hat{a}\right\rangle+|\beta|^{2}\right],
\end{aligned}
$$

em que $\left\langle: \Delta^{2} \hat{n}_{a}:\right\rangle$ é a variância do operador número do campo $\hat{a}$ em ordem normal ${ }^{5}$.

Explicitando a amplitude complexa $\beta=|\beta| e^{i \theta}$, a variância da subtração das fotocorrentes é reescrita como

$$
\Delta^{2} I_{-}=k^{2}\left(\left\langle\hat{a}^{\dagger} \hat{a}\right\rangle+|\beta|^{2}\right)+k^{2}\left|n_{b}\right|^{2}[\underbrace{\left\langle\left(\hat{a}^{\dagger} e^{i \theta}+\hat{a} e^{-i \theta}\right)^{2}\right\rangle}_{\left\langle\hat{X}_{\theta}^{2}\right\rangle}-\underbrace{\left\langle\left\langle\hat{a}^{\dagger}\right\rangle e^{i \theta}+\langle\hat{a}\rangle e^{-i \theta}\right\rangle^{2}}_{\left\langle\hat{X}_{\theta}\right\rangle^{2}}],
$$

onde notamos a presença da quadratura generalizada, $\hat{X}_{\theta}$, do campo $\hat{a}$. Podemos ainda escrever as variâncias em termos do valor médio das intensidades de cada campo, obtendo

$$
\begin{aligned}
& \Delta^{2} I_{-}=k\left(\left\langle I_{a}\right\rangle+\left\langle I_{b}\right\rangle\right)+k\left\langle I_{b}\right\rangle \Delta^{2} \hat{X}_{\theta} \\
& \Delta^{2} I_{+}=k^{2}\left\langle: \Delta^{2} \hat{n}_{a}:\right\rangle+k\left\langle I_{a}\right\rangle+k\left\langle I_{b}\right\rangle .
\end{aligned}
$$

O resultado acima nos mostra que as flutuações da subtração das intensidades têm uma relação direta com as flutuações da quadratura do campo $\hat{a}$. Variando a fase $\theta$ do campo coerente (oscilador local), temos acesso a todas as quadraturas do campo. Um caso particularmente interessante ocorre quando consideramos o modo do campo $\hat{b}$ como sendo o modo de vácuo, $|\beta\rangle=|0\rangle$. As expressões (2.6.17) se tornam

$$
\begin{aligned}
\Delta^{2} I_{-} & =k\left\langle I_{a}\right\rangle \\
\Delta^{2} I_{+} & =k\left\langle I_{a}\right\rangle+k^{2}\left\langle: \Delta^{2} \hat{n}_{a}:\right\rangle
\end{aligned}
$$

Para um estado coerente, as flutuações de intensidade são iguais ao valor médio do campo, o que caracteriza uma distribuição Poissoniana no número de fótons. Sendo assim, reconhecemos na expressão (2.6.18) o termo correspondente ao shot-noise. Na equação (2.6.19) notamos duas contribuições, uma devido ao shot-noise, primeiro termo do lado direito, e outra devido a ruídos característicos do estado analisado, segundo termo.

A forma usual de calibração do shot-noise se dá pela equação (2.6.18) com a obtenção do coeficiente linear $k$. Feito isso, os ruídos de uma dada medida são normalizados pela constante $k$ multiplicada pelo valor médio $\left\langle I_{a}\right\rangle$ do campo correspondente, o que equivale a

\footnotetext{
${ }^{5}$ Ordem normal é definida como aquela em que os operadores de criação são posicionados à esquerda dos operadores de aniquilação.
} 
normalizar $\Delta^{2} I_{-}$. Esse é o método mais utilizado para relativizar os ruídos ao shot-noise. Além disso, como a variância da subtração das fotocorrentes tem uma dependência linear com a intensidade do campo, a técnica serve como um teste para a eletrônica de detecção.

Outro método baseado na ideia acima consiste em medir os ruídos de interesse e normalizá-lo pelo shot-noise a cada medida. Faz-se isto calculando diretamente a razão $\Delta^{2} I_{+} / \Delta^{2} I_{-}$:

$$
\frac{\Delta^{2} I_{+}}{\Delta^{2} I_{-}}=\frac{k\left\langle: \Delta^{2} \hat{n}_{a}:\right\rangle}{\left\langle I_{a}\right\rangle}+1
$$

de onde se notam as contribuições do ruído do campo de interesse e do ruído quântico padrão, igual à unidade, dado que foi divido por ele mesmo.

Se reescrevermos $\left\langle: \Delta^{2} \hat{n}_{a}:\right\rangle$ em termos de $I_{a}$, obtemos

$$
\frac{\Delta^{2} I_{+}}{\Delta^{2} I_{-}}=\frac{\Delta^{2} I_{a}}{k I_{a}}=\Delta^{2} p
$$

onde a segunda igualdade se refere à equação (2.2.15). Este resultado nos mostra que a razão entre as variâncias dos sinais de soma e subtração das fotocorrentes nos fornece o ruído de amplitude do campo já normalizado pelo shot-noise.

Ao lidarmos com medidas de flutuações do campo, devemos levar em consideração todas as fontes de ruído que serão registrados no ato da medida. Basicamente, ao incidir um campo em um fotodetector, o ruído total $S_{T}$ medido também tem contribuições da eletrônica do aparato experimental. Explicitamente,

$$
S_{T}=\Delta^{2} I(t)+\underbrace{\Delta^{2} I(t)_{e s c}+\Delta^{2} I(t)_{t h}+\Delta^{2} I(t)_{a m p}}_{\Delta^{2} I(t)_{e l e}},
$$

onde o primeiro termo é o ruído intrínseco da luz e os demais são diferentes tipos de ruído eletrônico, $\Delta^{2} I(t)_{\text {ele }}$. O primeiro deles, $\Delta^{2} I(t)_{\text {esc }}$ [Spiegel 1980], refere-se a falsas detecções, pois mesmo na ausência de luz os fotodetectores produzem algum tipo de sinal, chamado de corrente escura. O segundo, $\Delta^{2} I(t)_{t h}$, está relacionado ao ruído térmico, conhecido também como ruído de Johnson-Nyquist [Vetterling 1980, Spiegel 1980], devido à agitação dos elétrons nos elementos que formam a eletrônica (resistores, por exemplo). O último deles, $\Delta^{2} I(t)_{a m p}$, surge por causa da amplificação do sinal. Portanto, antes de qualquer medida de shot-noise, é imprescindível a medida do ruído eletrônico do sistema para que seja subtraído de todas as aquisições e o ruído resultante seja somente o de interesse [Bachor 1998].

Mostramos nesta seção como é medido o ruído quântico padrão, referência para identificar características puramente quânticas nos estados de interesse. Também exibimos como os demais ruídos podem ser normalizados pelo shot-noise, de modo que, se iguais a um, serão os mesmos encontrados em estados coerentes, menores que um indicarão compressão e se maiores mostrarão que o estado apresenta excesso de ruído. Contudo, falta ainda apresentar como podemos acessar flutuações de diferentes quadraturas, assunto que será apresentado a seguir. 


\subsection{Medidas das flutuações das quadraturas}

Todas as medidas do campo são realizadas com fotodetectores que medem diretamente apenas informações sobre a amplitude do campo, uma vez que esta é proporcional à intensidade do mesmo. Para que seja possível acessar as flutuações de fase devemos utilizar técnicas interferométricas, misturando o campo de interesse com um oscilador local ótico, de modo que a fase relativa entre eles seja bem controlada. Discutiremos nas próximas linhas a detecção homodina, descrita anteriormente, e a auto-homodina que faz uso de cavidades óticas vazias, técnicas mais amplamente empregadas.

\subsubsection{Medidas das quadraturas: detecção homodina}

A forma usual de realizar medidas de quadraturas de um campo consiste em usar um divisor de feixes (figura 2.4), tal como discutido na seção 2.6.1. Ao calcularmos o valor médio da subtração das fotocorrentes (equação 2.6.13) para o estado $|\Phi\rangle=\left|\Phi_{a}\right\rangle \otimes|\beta\rangle$, obtemos

$$
\begin{aligned}
\left\langle I_{-}\right\rangle & =k\left(\left\langle\hat{a}^{\dagger}\right\rangle \beta+\langle\hat{a}\rangle \beta^{*}\right) \\
& =k|\beta|\left(\left\langle\hat{a}^{\dagger}\right\rangle e^{-i \theta}+\langle\hat{a}\rangle e^{i \theta}\right) \\
& =k|\beta|\left\langle\hat{X}_{\theta}\right\rangle .
\end{aligned}
$$

Ou seja, o valor esperado da quadratura generalizada $\hat{X}_{\theta}$ é dado pelo valor médio da subtração dos campos de saída de um divisor de feixes, bastando variar a fase do oscilador local (campo $\hat{b}$ ) para obter a quadratura desejada. No domínio temporal, essa descrição é completa e fornece toda informação necessária para reconstrução do estado quântico estudado, algo que pode ser visto na descrição de experimentos envolvendo tomografia de estados quânticos [Leonhardt 1997]. Contudo, no domínio espectral, onde lidamos com medidas de modos simetricamente localizados no entorno da portadora central do campo, esta não é a única fase que desempenha um papel importante no processo de medida.

$\mathrm{Na}$ subseção 2.4.5, após estudar a fotocorrente no domínio da frequência, vimos o efeito da variação da fase relativa entre o oscilador local ótico e eletrônico nas medidas de flutuação da fotocorrente. Em termos dos modos das bandas laterais, é possível mostrar que, desenvolvendo a equação (2.4.31) com o auxílio das equações (2.4.25) e (2.4.26), a seguinte expressão para a potência de ruído é obtida,

$$
\begin{gathered}
S_{D H}=\frac{1}{2} \cos ^{2} \varphi\left(\Delta^{2} \hat{p}_{s}+\Delta^{2} \hat{q}_{a}\right)+\frac{1}{2} \operatorname{sen}^{2} \varphi\left(\Delta^{2} \hat{p}_{a}+\Delta^{2} \hat{q}_{s}\right) \\
+\frac{1}{2} \operatorname{sen} 2 \varphi\left(C\left(\hat{p}_{s}, \hat{q}_{s}\right)-C\left(\hat{p}_{a}, \hat{q}_{a}\right)\right) .
\end{gathered}
$$

Quando consideramos o caráter estacionário do estado quântico, a expressão acima pode ser simplificada. Para isso, utilizamos as entradas da matriz de covariâncias $\mathbf{V}_{s / a}$ (equação 2.4.39) a fim de escrever

$$
S_{H D}=\cos ^{2} \varphi \alpha+\operatorname{sen}^{2} \varphi \beta+\operatorname{sen} 2 \varphi \gamma .
$$


Nesta expressão, nota-se a ausência do termo $\delta$ da matriz $\mathbf{V}_{s / a}$, fato que caracteriza a detecção homodina como incompleta na reconstrução do estado mais geral do campo. Porém, se garantirmos que o termo $\delta=C\left(\hat{p}_{s} \hat{p}_{a}\right)=C\left(\hat{q}_{s} \hat{q}_{a}\right)$ é nulo, essa limitação é irrelevante, algo difícil de ser determinado na prática.

Dando continuidade, a fim de revelar o que de fato não é acessado, escrevemos explicitamente a expressão para $\delta$ em termos dos elementos da matriz $\mathbf{V}_{-\Omega / \Omega}$, escrita na base dos modos das bandas laterais, obtendo

$$
2 \delta=\left(\Delta^{2} \hat{p}_{\Omega}+\Delta^{2} \hat{q}_{\Omega}\right)-\left(\Delta^{2} \hat{p}_{-\Omega}+\Delta^{2} \hat{q}_{-\Omega}\right)
$$

Nesta expressão, a grandeza $\delta$ tem seu conteúdo físico explícito: diferença de energia entre as bandas laterais. Portanto, é razoável dizer que a detecção homodina é uma técnica de modo único, dada a completa indeterminação de propriedades individuais dos modos $\Omega$ e $-\Omega$, tratando-os de forma completamente simétrica e indistinguível. Neste sentido, o campo é examinado como uma aproximação de um único modo efetivo.

Por se tratar de um método que envolve interferência entre os campos que interagem em um divisor de feixes, o comprimento de onda dos mesmos deve ser igual, impondo grandes restrições em experimentos que lidam com feixes de comprimento de onda distintos, pois muitas vezes é bastante complicado implementar medidas com vários osciladores locais, um para cada campo.

Para contornar essa situação, usamos em nosso laboratório a detecção auto-homodina. Essa técnica faz uso de cavidades óticas vazias para girar a elipse de ruído representada na figura 2.3.

\subsubsection{Cavidade ótica vazia}

A detecção auto-homodina tira proveito das propriedades dispersivas de cavidades óticas vazias que atuam sobre todos os modos do campo, não se limitando à defasagem do oscilador local ótico a exemplo do que acontece na detecção homodina. Para entender melhor o mecanismo de funcionamento da técnica é necessário apontar as principais características da sua ferramenta principal, a cavidade ótica.

Consideremos uma cavidade ótica Fabry-Perot formada por um espelho de acoplamento de reflexão $R_{1}$ e um espelho de saída altamente refletor $R_{2} \approx 1$ separados por uma distância $L / 2$, conforme apresentado na figura 2.5. A transmissão e a reflexão dos espelhos são dadas pelas relações $\left|t_{j}\right|^{2}=T_{j}$ e $\left|r_{j}\right|^{2}=R_{j}(j=1,2)$, onde $r_{j}$ e $t_{j}$ são coeficientes complexos. A fim de facilitar os cálculos, a fase do campo incidente é escolhida nula na posição do espelho de acoplamento. Os campos ilustrados na figura 2.5 se relacionam da seguinte maneira,

$$
\begin{array}{lll}
E_{c}=t_{1} E_{\mathrm{in}}+r_{1} E_{\mathrm{c}^{\prime}}, & & E_{c^{\prime}}=r_{2} E_{c} e^{(i k L / 2),}, \\
E_{R}=-r_{1} E_{\mathrm{in}}+t_{1} E_{\mathrm{c}^{\prime}} & & E_{T}=t_{2} E_{c} e^{(i k L)} .
\end{array}
$$




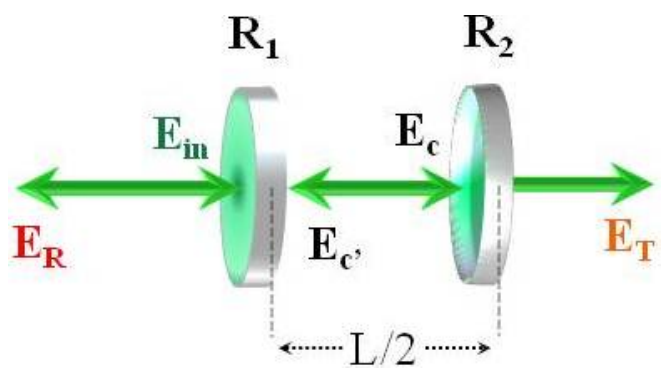

Figura 2.5: Cavidade Fabry-Perot

O campo $E_{T}$ transmitido pela cavidade é dado pela fração do campo $E_{c}$ transmitida pelo espelho de saída 2 , enquanto o campo refletido $E_{R}$ é dado pela soma da fração transmitida de $E_{c^{\prime}}$ com a fração do campo incidente $E_{i n}$, refletida pelo espelho de acoplamento. Com estas informações, é razoavelmente simples mostrar que as amplitudes clássicas dos campos $E_{T}$ e $E_{R}$ se relacionam com a amplitude do campo incidente da seguinte forma

$$
E_{R}=r(L) E_{\text {in }} \quad \text { e } \quad E_{T}=t(L) E_{i n}
$$

onde os coeficientes de reflexão e transmissão da cavidade têm suas expressões dadas por

$$
r(L)=-\frac{r_{1}-r_{2} \exp (i k L)}{1-r_{1} r_{2} \exp (i k L)} \quad \text { e } \quad t(L)=\frac{t_{1} t_{2} \exp (i k L / 2)}{1-r_{1} r_{2} \exp (i k L)} .
$$

Calculando as expressões para a transmissão $T$ e reflexão $R$ da cavidade, dadas respectivamente por $|r(L)|^{2}$ e $|t(L)|^{2}$, verifica-se que a condição de ressonância é satisfeita para comprimentos da cavidade iguais a $n \lambda / 2$, onde $\lambda$ é o comprimento de onda da luz incidente e $n$ um número inteiro. Nesta condição, as expressões para a transmissão máxima $\left(T_{\max }\right)$ e reflexão mínima $\left(R_{\min }\right)$ da cavidade são escritas como

$$
\begin{aligned}
& R_{\text {min }}=\frac{\left(\sqrt{R_{1}}-\sqrt{R_{2}}\right)^{2}}{\left(1-\sqrt{R_{1} R_{2}}\right)^{2}} \\
& T_{\text {max }}=1-R_{\text {min }} .
\end{aligned}
$$

Como veremos, essas grandezas são de grande utilidade por aparecerem explicitamente nas expressões das amplitudes de ruído dos campos após interagirem com a cavidade ótica.

Outros parâmetros bastante úteis, responsáveis pela caracterização das cavidades, são: a largura de banda $\delta \nu_{c}$ ou bandwidth (BW), definida como a largura em frequência à meia altura de um pico de ressonância da cavidade, o intervalo espectral livre $\Delta \nu_{c}$ ou free spectral range (FRS), dado pela separação em frequência entre dois picos de ressonância consecutivos, associado ao tempo que um fóton leva para dar uma volta na cavidade e a finesse $F$, que nos dá a qualidade da cavidade em termos de armazenamento de luz. A finesse é definida como sendo a razão entre o intervalo espectral livre e a largura de banda da cavidade, cuja expressão é [Grynberg 2010]

$$
F=\pi \frac{\left(R_{1} R_{2}\right)^{1 / 4}}{1-\sqrt{R_{1} R_{2}}}
$$


Para cavidades com alta finesse $\left(T_{1}, T_{2} \ll 1\right)$, é possível simplificar consideravelmente a equação anterior por meio de expansões simples, a fim de obter

$$
F \approx \frac{2 \pi}{T_{1}+T_{2}}
$$

que quantifica de forma direta as perdas pelos espelhos, dada pela quantidade $\left(T_{1}+T_{2}\right)$. Visando deixar ainda mais claro o significado físico da finesse, calculamos a razão entre as intensidades do campo $\left|E_{c} / E_{i n}\right|^{2}$ na ressonância, considerando perfeito o espelho $T_{2}$ $\left(T_{2} \approx 0\right)$ e utilizando a equação $(2.7 .13)$, resultando em

$$
\left|\frac{E_{c}}{E_{\text {in }}}\right|^{2} \approx \frac{2}{\pi} F
$$

Vê-se que uma maior finesse implica maior intensidade do campo intracavidade.

$\mathrm{Na}$ descrição da técnica auto-homodina trataremos os efeitos da cavidade sobre o campo através do parâmetro controlável $\Delta$, definido como

$$
\Delta_{\nu_{0}}=\frac{\left(\nu_{0}-\nu_{c}\right)}{\delta \nu_{c}}
$$

que quantifica quão longe da ressonância da cavidade $\nu_{c}$ está a frequência do campo $\nu_{0}$. A dessintonia, por questão de simplicidade, é normalizada pela largura de banda da cavidade, o que a torna adimensional. Como a frequência do campo é fixa, a variação da dessintonia é realizada pelo controle de uma cerâmica piezoelétrica (PZT) conectada ao suporte de um dos espelhos da cavidade, que se expande ou contrai dependendo do sinal da voltagem aplicada, alterando o comprimento da cavidade e assim sua frequência de ressonância.

Levando em conta o parâmetro $\Delta_{\nu_{0}}$, as expressões para a reflexão e transmissão (equação 2.7.9) podem ser reescritas como

$$
r\left(\Delta_{\nu_{0}}\right)=-\frac{r_{1}-r_{2} \exp \left(i 2 \pi \Delta_{\nu_{0}} / F\right)}{1-r_{1} r_{2} \exp \left(i 2 \pi \Delta_{\nu_{0}} / F\right)} \quad \text { e } \quad t\left(\Delta_{\nu_{0}}\right)=\frac{t_{1} t_{2} \exp \left(i 2 \pi \Delta_{\nu_{0}} / 2 F\right)}{1-r_{1} r_{2} \exp \left(i 2 \pi \Delta_{\nu_{0}} / F\right)}
$$

Na figura 2.6 estão representados a reflexão $R\left(\Delta_{\nu_{0}}\right)=\left|r\left(\Delta_{\nu_{0}}\right)\right|^{2}$ e a fase $e^{i \theta_{R}}=$ $r\left(\Delta_{\nu_{0}}\right) /\left|r\left(\Delta_{\nu_{0}}\right)\right|$ do campo refletido em função da dessintonia da cavidade. Note que à medida que a dessintonia varia, passando pela ressonância, $\Delta_{\nu_{0}}=0$, a fase do campo refletido varia na forma de arcotangente de 0 a $2 \pi$. Como era de se esperar, a reflexão possui valor mínimo na ressonância, implicando máxima transmissão, dada a relação $\left|r\left(\Delta_{\nu_{0}}\right)\right|^{2}+\left|t\left(\Delta_{\nu_{0}}\right)\right|^{2}=1$. Veremos agora como estes resultados possibilitam o acesso às quadraturas amplitude e fase do campo.

\subsubsection{Detecção Auto homodina}

A técnica auto-homodina foi primeiramente usada por Levenson et al. [Levenson 1985] e Galatola et al. [Galatola 1991] em medidas de estados com compressão de ruído. O 


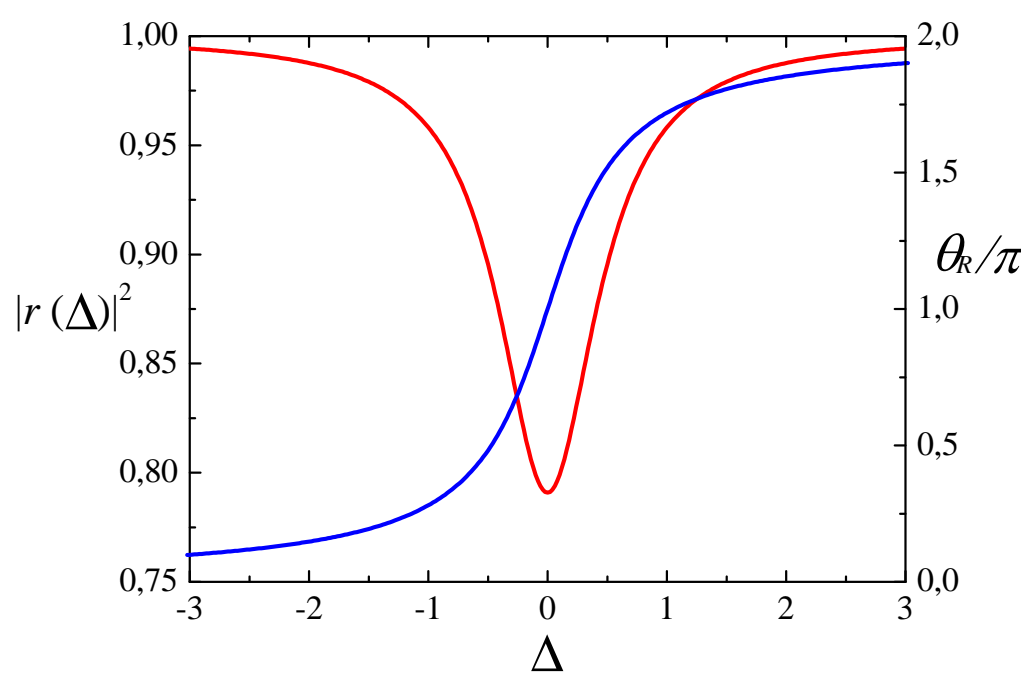

Figura 2.6: Módulo ao quadrado (curva em vermelho) e fase (curva em azul) de $r\left(\Delta_{\nu_{0}}\right)$ em função da dessintonia da cavidade. Foram utilizados $R_{1}=95 \%$ e $R_{2}=99,7 \%$, valores compatíveis com as especificações dos espelhos utilizados em nossos experimentos.

uso de cavidades óticas viabiliza a medida de flutuações da quadratura fase projetandoas em flutuações de amplitude, por meio da variação de fase do campo refletido, imposta pela dessintonia. Uma descrição bastante detalhada pode ser vista nas referências [Villar 2007b, Villar 2008].

De acordo com Galatola et al. [Galatola 1991] e Villar [Villar 2008], uma cavidade ótica com alta finesse transforma o operador de aniquilação associado a um modo do campo de frequência $\Omega$ em

$$
\hat{a}_{\Omega} \longrightarrow r\left(\Delta_{\Omega}\right) \hat{a}_{\Omega}+t\left(\Delta_{\Omega}\right) \hat{v}_{\Omega},
$$

onde as perdas do campo associadas a processos espúrios dentro da cavidade foram modeladas pela inclusão do operador $\hat{v}_{\Omega}$, visto como um modo de vácuo que se acopla ao modo intracavidade. Isso significa substituir os modos que se perdem no processo de reflexão por $\hat{v}_{\Omega}$. Após interagir com a cavidade, o campo correspondente à portadora central $\beta=|\beta| e^{-i \theta}$ sofre a transformação

$$
|\beta| e^{-i \theta} \longrightarrow|\beta| r(\Delta), \quad \Delta_{\omega_{0}} \equiv \Delta,
$$

onde a fase $\theta$ é considerada nula, sem perder em generalidade. É importante frisar que na técnica auto-homodina a portadora central funciona como um oscilador local análogo ao utilizado na detecção homodina usual. Por estar inclusa no feixe de interesse, justifica-se a presença do prefixo "auto" em sua denominação.

Substituindo essas transformações na expressão da fotocorrente (2.4.23) obtida pela análise de Fourier, obtemos

$$
\begin{aligned}
\hat{J}_{\Omega}(\Delta) & =R(\Omega, \Delta) \hat{a}_{\Omega}+R^{*}(-\Omega, \Delta) \hat{a}_{-\Omega}^{\dagger}+T(\Omega, \Delta) \hat{v}_{\Omega}+T^{*}(-\Omega, \Delta) \hat{v}_{-\Omega} \\
& =\hat{J}_{\text {cos }}+i \hat{J}_{\text {sen }}+\hat{J}_{\text {vac }}
\end{aligned}
$$


com coeficientes

$$
R(\Omega, \Delta)=\frac{r^{*}(\Delta)}{|r(\Delta)|} r\left(\Delta+\Omega / \delta \nu_{c}\right) \quad \text { e } \quad T(\Omega, \Delta)=\frac{r^{*}(\Delta)}{|r(\Delta)|} t\left(\Delta+\Omega / \delta \nu_{c}\right) .
$$

Seguindo o raciocínio da seção 2.4.3 decompomos a fotocorrente $\hat{J}$ em suas quadraturas $\hat{J}_{\text {cos }}$ e $\hat{J}_{\text {sen }}$, além de tornar explícitas as contribuições advindas do vácuo, que aparecem multiplicando os coeficientes $T(\Omega, \Delta)$. Dando sequência, vamos explicitar as quadraturas $\hat{J}_{\text {cos }}$ e $\hat{J}_{\text {sen }}$ da fotocorrente definindo funções reais $x_{ \pm}$e $y_{ \pm}$de $\Delta$ a partir dos coeficientes acima,

$$
\begin{gathered}
x_{+}+i y_{+}=\left[R(\Omega, \Delta)+R^{*}(-\Omega, \Delta)\right] / 2 \equiv g_{+}, \\
x_{-}+i y_{-}=i\left[R(\Omega, \Delta)-R^{*}(-\Omega, \Delta)\right] / 2 \equiv g_{-} .
\end{gathered}
$$

Reescrevendo a equação (2.7.19) em termos dos operadores $\hat{p}_{ \pm \Omega}$ e $\hat{q}_{ \pm \Omega}$ e empregando em seguida as relações (2.7.22), podemos ver que na base $\left\{\hat{p}_{s}, \hat{q}_{s}, \hat{p}_{a}, \hat{q}_{a}\right\}$, as quadraturas da fotocorrente definidas como $\hat{J}_{\Omega}(\Delta)=\sqrt{2}\left(\hat{J}_{\mathrm{cos}}+i \hat{J}_{\mathrm{sen}}+\hat{J}_{\text {vac }}\right)$ têm a forma

$$
\begin{aligned}
\hat{J}_{\cos }(\Delta) & =\hat{x}_{+} \hat{p}_{s}-\hat{y}_{+} \hat{q}_{a}+\hat{x}_{-} \hat{q}_{s}+\hat{y}_{-} \hat{p}_{a} \\
\hat{J}_{\mathrm{sen}}(\Delta) & =\hat{x}_{+} \hat{q}_{a}+\hat{y}_{+} \hat{p}_{s}-\hat{x}_{-} \hat{p}_{a}+\hat{y}_{-} \hat{q}_{s} .
\end{aligned}
$$

Suprimiremos as contribuições dos estados de vácuo por questão de simplicidade. Portanto, o tratamento a seguir corresponde ao caso ideal, livre de perdas.

É fácil verificar que quadraturas da fotocorrente podem ser medidas concomitantemente pois $\left[\hat{J}_{\cos }, \hat{J}_{\text {sen }}\right]=0$. Notamos ainda que a equação anterior sugere que a detecção auto-homodina pode, em princípio, ser uma técnica mais completa dado que as funções que multiplicam as quadraturas dos modos $s$ e $a$ são distintas.

Segue dessas expressões que o espectro de ruído (equação 2.4.31) da fotocorrente gerada pelo campo depois da interação com a cavidade é dado por

$$
\begin{aligned}
& S_{A H}=\frac{1}{2}\left|g_{+}\right|^{2}\left(\Delta^{2} \hat{p}_{s}+\Delta^{2} \hat{q}_{a}\right)+\frac{1}{2}\left|g_{-}\right|^{2}\left(\Delta^{2} \hat{p}_{a}+\Delta^{2} \hat{q}_{s}\right)+ \\
& +\quad\left[C\left(\hat{p}_{s} \hat{q}_{s}\right)-C\left(\hat{p}_{a} \hat{q}_{a}\right)\right] \operatorname{Re}\left\{g_{+}^{*} g_{-}\right\}+\left[C\left(\hat{p}_{s} \hat{p}_{a}\right)+C\left(\hat{q}_{s} \hat{q}_{a}\right)\right] \operatorname{Im}\left\{g_{+}^{*} g_{-}\right\}
\end{aligned}
$$

Neste ponto, podemos considerar o efeito da estacionariedade da fotocorrente ou, de modo equivalente, a ausência de travamento de fase relativa entre o oscilador local ótico e eletrônico. Nessas condições, a simetria existente entre os operadores exposta nas matrizes de covariâncias (2.4.39) impõe que o espectro $S_{A H}$ seja da forma,

$$
S_{A H}=c_{\alpha} \alpha+c_{\beta} \beta+c_{\gamma} \gamma+c_{\delta} \delta
$$

onde $c_{\alpha}=\left|g_{+}\right|^{2}, c_{\beta}=\left|g_{-}\right|^{2}$ e $c_{\gamma}+i c_{\delta}=g_{+}^{*} g_{-}$. A comparação direta de $S_{D H}$ com $S_{A H}$ nos mostra que o uso de cavidades óticas traz mais informações sobre a estrutura do campo do que a técnica de detecção homodina. Isto pode ser visto pela presença da 
quantidade $\delta$ na expressão de $S_{A H}$, fato que não ocorre na expressão de $S_{D H}$ (equação 2.7.4). Uma justificativa para esta diferença é o caráter dispersivo da cavidade ótica vazia, pois, como veremos, cada modo interage com a cavidade de forma independente, alterando a fase relativa entre modo que está passando pela ressonância com a cavidade e os demais refletidos. Contrariamente, a detecção homodina não faz distinção entre os modos das bandas laterais, tratando-os de modo simétrico.

Contudo, conforme visto, a grandeza $\delta$ tem como significado físico uma diferença de energia entre as bandas laterais do campo, o que implica igualdade entre as duas técnicas, caso a diferença de energia não exista. Dito de outra forma, se o parâmetro $\delta$ for nulo, as duas técnicas conseguem extrair as mesmas informações do campo.

A análise dos estados quânticos produzidos em nosso sistema é feita supondo que o parâmetro $\delta$ é nulo, ou seja, lidamos com os campos produzidos numa aproximação denominada aqui como aproximação de modo único, pois não fazemos distinção entre as bandas laterais.

Justificamos isto analisando, por exemplo, o operador $\hat{p}(\Omega)$ dado na equação (2.2.16) para $\theta=\varphi$. Em nossa descrição, este operador representa a quadratura amplitude de um campo. Podemos reescrevê-lo considerando sua forma no espaço de fase da função de Wigner, onde os operadores de criação e aniquilação são substituídos por amplitudes complexas,

$$
p(\Omega)=\alpha(\Omega) e^{-i \varphi}+\alpha^{*}(-\Omega) e^{i \varphi}
$$

Utilizando a tabela de correspondências 2.3.1 verificamos que a equação acima carrega consigo informações sobre as flutuações das duas bandas laterais do campo. Dessa forma, podemos dizer que estamos lidando com um modo único somente quando consideramos perfeita simetria entre os modos de frequência $\Omega$ e $-\Omega$, ou seja, diferença de energia entre os modos nula. A seguir, analisaremos intuitivamente o efeito da cavidade ótica sobre as flutuações do campo.

\section{Rotação da elipse de ruído}

Iniciaremos a discussão observando as expressões para as flutuações das quadraturas amplitude e fase no domínio da frequência,

$$
\begin{aligned}
& \delta p(\Omega)=e^{-i \varphi} \delta \alpha(\Omega)+e^{i \varphi} \delta \alpha^{*}(-\Omega), \\
& \delta q(\Omega)=-i\left[e^{-i \varphi} \delta \alpha(\Omega)-e^{i \varphi} \delta \alpha^{*}(-\Omega)\right] .
\end{aligned}
$$

Recordamos que $\Omega$ é a frequência de análise, ajustada eletronicamente pelo oscilador local eletrônico. As flutuações observadas são referentes à essa componente. De modo mais preciso, estamos observando as flutuações nas bandas laterais $\omega_{0} \pm \Omega$ no entorno da portadora central da frequência ótica $\omega_{0}$. As quadraturas são definidas tomando como referência a fase $\varphi$ do oscilador ótico (portadora). 
A projeção das flutuações de fase em flutuações de amplitude realizada pela cavidade ótica pode ser compreendida por meio de exemplo simples. Suponhamos ser possível manipular independentemente a fase de cada uma das componentes de frequência presentes na expressão da quadratura $\delta p(\Omega)$ : portadora central e bandas laterais, rotuladas, por simplicidade, por $\pm \Omega$. Dada essa possibilidade, vamos ver o efeito da inclusão de uma fase $\theta$ controlável à cada uma das componentes. Como exemplo, consideremos a banda lateral $\Omega$ da equação(2.7.28):

$$
\begin{aligned}
\delta p(\Omega) & \longrightarrow e^{i \theta} e^{-i \varphi} \delta \alpha(\Omega)+e^{i \varphi} \delta \alpha^{*}(-\Omega), \\
& =e^{i \theta / 2}\left[e^{-i(\varphi-\theta / 2)} \delta \alpha(\Omega)+e^{i(\varphi-\theta / 2)} \delta \alpha^{*}(-\Omega)\right] .
\end{aligned}
$$

Alterando continuamente o ângulo $\theta$ de 0 a $2 \pi$, notamos uma conversão de flutuação de fase para flutuação de amplitude, que ocorre exatamente para $\theta= \pm \pi$, a menos de uma fase global. O mesmo raciocínio vale para a banda lateral negativa.

Uma análise similar pode ser feita alterando a fase da portadora, o que equivale a reescrever $\varphi \longrightarrow \varphi+\theta$. Segue então

$$
\delta p(\Omega) \longrightarrow e^{-i(\varphi+\theta)} \delta \alpha(\Omega)+e^{i(\varphi+\theta)} \delta \alpha^{*}(-\Omega)
$$

Mais uma vez, variando continuamente a fase $\theta$ obtemos a conversão exata de quadratura fase para quadratura amplitude quando $\theta=\pi / 2$ ou $\theta=3 \pi / 2$.

Na figura 2.3, ilustramos as amplitudes das flutuações por uma elipse de ruído ${ }^{6}$, um contorno das mesmas no espaço de fase da função de Wigner. A cavidade ótica é o dispositivo que adiciona e controla a fase $\theta$ através da variação da dessintonia. Em outras palavras, a fase relativa entre as frequências do campo é alterada à medida que variamos a dessintonia da cavidade. Na figura 2.8 mostramos graficamente o que ocorre com a elipse de ruído quando a cavidade passa pela ressonância com cada uma das frequências envolvidas.

Agora, vamos escrever a expressão matemática para a potência de ruído de $p(\Omega)$ após a interação do campo com a cavidade. O campo refletido pela cavidade está relacionado com o campo incidente e com os modos de vácuo, que se incluem por conta das perdas, através da expressão

$$
\alpha_{R}\left(\nu^{\prime}\right)=r\left(\Delta+\nu^{\prime}\right) \alpha_{i n}\left(\nu^{\prime}\right)+t\left(\Delta+\nu^{\prime}\right) \alpha_{v}\left(\nu^{\prime}\right)
$$

sendo a reflexão e transmissão dadas pela equação (2.7.16), com os devidos argumentos, e $\nu^{\prime}=\Omega / \delta \nu_{c}$ a frequência de análise relativa à largura de banda da cavidade. Note que, além de depender da dessintonia, a reflexão e transmissão dependem de $\nu^{\prime}$, justificando

\footnotetext{
${ }^{6}$ A elipse de ruído representa as flutuações encontradas em estados comprimidos. No caso de estados coerentes as flutuações são ilustradas por uma circunferência, pois o ruído é igualmente distribuído em suas quadraturas.
} 


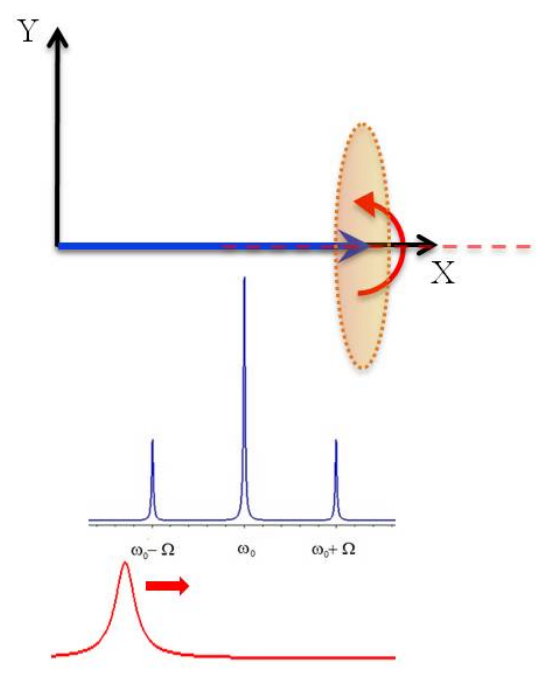

(a)

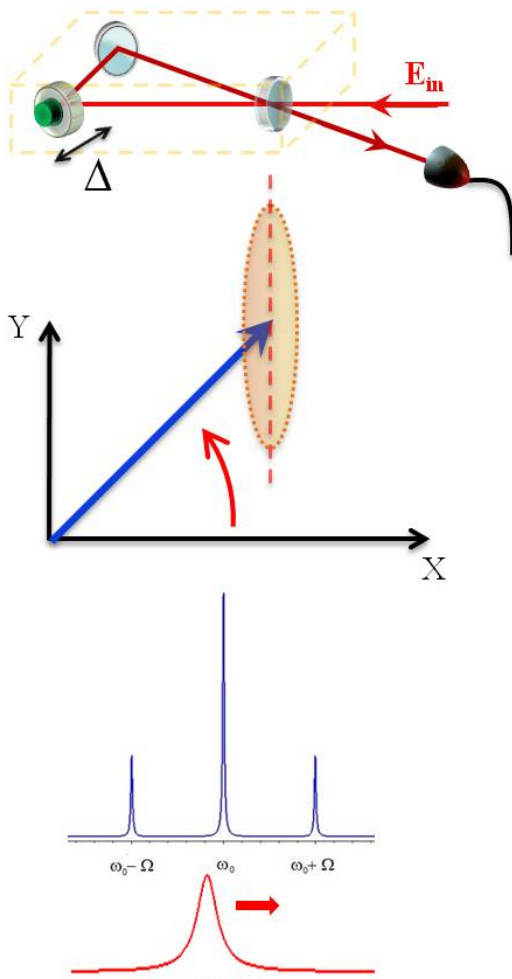

(b)

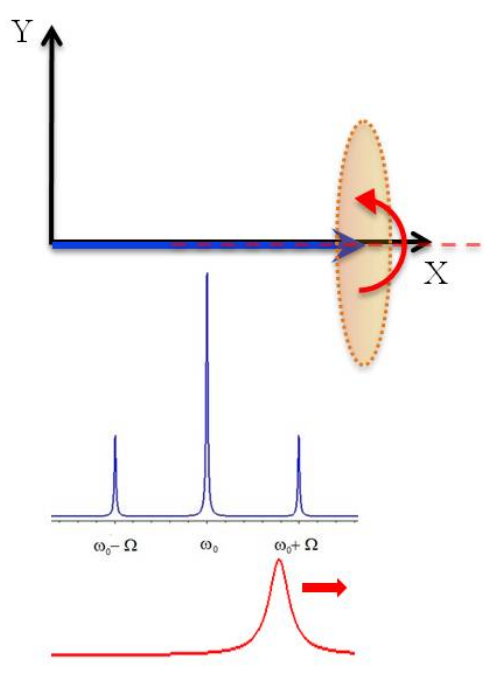

(c)

Figura 2.7: Representação gráfica da conversão do ruído de fase em ruído de amplitude, propiciada pelas características dispersivas de ressonância de uma cavidade ótica vazia, ilustrada na parte superior da figura. Em (a) a frequência "vista" pela cavidade corresponde à banda lateral negativa, causando um giro da elipse de ruído sem modificar a fase da portadora. Em (b) a cavidade passa pela ressonância com a frequência do oscilador local ótico, a portadora central. Nesta situação quem gira é o vetor correspondente ao valor médio do campo, mantendo fixa a posição da elipse. O efeito sobre a banda lateral positiva em (c) é o mesmo obtido em (a). Para facilitar a compreensão inserimos uma linha tracejada em vermelho que aponta a direção que permanece fixa com a variação da fase.

a alteração da fase relativa entre a frequência em questão e as demais. A fase relativa é dada por $e^{-i \theta_{R}(\Delta)}=\frac{r\left(\Delta+\nu^{\prime}\right)}{\left|r\left(\Delta+\nu^{\prime}\right)\right|}$.

Aplicando a equação anterior à portadora central e às bandas laterais, obtemos

$$
\begin{gathered}
\alpha_{R}(0)=r(\Delta) \alpha_{i n}(0) \\
\delta \alpha_{R}\left(\nu^{\prime}\right)=r\left(\Delta+\nu^{\prime}\right) \delta \alpha_{i n}\left(\nu^{\prime}\right)+t\left(\Delta+\nu^{\prime}\right) \delta \alpha_{v}\left(\nu^{\prime}\right) \\
\delta \alpha_{R}^{*}\left(-\nu^{\prime}\right)=r^{*}\left(\Delta-\nu^{\prime}\right) \delta \alpha_{i n}^{*}\left(-\nu^{\prime}\right)+t^{*}\left(\Delta-\nu^{\prime}\right) \delta \alpha_{v}^{*}\left(-\nu^{\prime}\right),
\end{gathered}
$$

onde escolhemos a frequência $\omega_{0}$ como referência para as bandas laterais, de forma que $\omega_{0} \pm \Omega \rightarrow \pm \Omega$.

Substituindo as exponenciais da equação (2.7.28) por $\alpha(\Omega) /|\alpha(\Omega)|$, escrevemos a qua- 
dratura amplitude do campo refletido como

$$
\delta p_{R}\left(\nu^{\prime}\right)=\frac{\alpha_{R}^{*}}{\left|\alpha_{R}\right|} \delta \alpha_{R}\left(\nu^{\prime}\right)+\frac{\alpha_{R}}{\left|\alpha_{R}\right|} \delta \alpha_{R}^{*}\left(-\nu^{\prime}\right) .
$$

Fazendo a inversão das equações (2.7.28) e (2.7.29) e substituindo em (2.7.37), juntamente com (2.7.34)-(2.7.36), obtemos

$$
\begin{aligned}
\delta p_{R}\left(\nu^{\prime}\right)= & g_{p}\left(\Delta, \nu^{\prime}\right) \delta p_{i n}\left(\nu^{\prime}\right)+i g_{q}\left(\Delta, \nu^{\prime}\right) \delta q_{i n}\left(\nu^{\prime}\right)+g_{v p}\left(\Delta, \nu^{\prime}\right) \delta v_{p}\left(\nu^{\prime}\right) \\
& +i g_{v q}\left(\Delta, \nu^{\prime}\right) \delta v_{q}\left(\nu^{\prime}\right)
\end{aligned}
$$

em que $\delta v=e^{i \varphi}\left(\delta v_{p}+i \delta v_{q}\right) / 2$ é a flutuação do vácuo, e

$$
\begin{aligned}
g_{p}\left(\Delta, \nu^{\prime}\right) & =\frac{1}{2}\left[\mathrm{e}^{-i \theta_{R}(\Delta)} r\left(\Delta+\nu^{\prime}\right)+\mathrm{e}^{i \theta_{R}(\Delta)} r^{*}\left(\Delta-\nu^{\prime}\right)\right], \\
g_{q}\left(\Delta, \nu^{\prime}\right) & =\frac{1}{2}\left[\mathrm{e}^{-i \theta_{R}(\Delta)} r\left(\Delta+\nu^{\prime}\right)-\mathrm{e}^{i \theta_{R}(\Delta)} r^{*}\left(\Delta-\nu^{\prime}\right)\right], \\
g_{v p}\left(\Delta, \nu^{\prime}\right) & =\frac{1}{2}\left[\mathrm{e}^{-i \theta_{R}(\Delta)} t\left(\Delta+\nu^{\prime}\right)+\mathrm{e}^{i \theta_{R}(\Delta)} t^{*}\left(\Delta-\nu^{\prime}\right)\right], \\
g_{v q}\left(\Delta, \nu^{\prime}\right) & =\frac{1}{2}\left[\mathrm{e}^{-i \theta_{R}(\Delta)} t\left(\Delta+\nu^{\prime}\right)-\mathrm{e}^{i \theta_{R}(\Delta)} t^{*}\left(\Delta-\nu^{\prime}\right)\right] .
\end{aligned}
$$

Utilizando a equação (2.4.29), calculamos o espectro de ruído para a quadratura amplitude do feixe refletido

$$
S_{R}\left(\Delta, \nu^{\prime}\right)=\left|g_{p}\left(\Delta, \nu^{\prime}\right)\right|^{2} S_{p}\left(\nu^{\prime}\right)+\left|g_{q}\left(\Delta, \nu^{\prime}\right)\right|^{2} S_{q}\left(\nu^{\prime}\right)+\left|g_{v p}\left(\Delta, \nu^{\prime}\right)\right|^{2}+\left|g_{v q}\left(\Delta, \nu^{\prime}\right)\right|^{2},
$$

$\operatorname{com} S_{v p}\left(\nu^{\prime}\right)=S_{v q}\left(\nu^{\prime}\right)=1$. Lembramos que os valores de $S_{v p}\left(\nu^{\prime}\right)$ e $S_{v q}\left(\nu^{\prime}\right)$ são iguais à unidade por se tratarem de ruídos do estado de vácuo, um autoestado do operador aniquilação e, portanto, um estado coerente, que fornece nossa referência de ruído. Observe que $S_{R}\left(\Delta, \nu^{\prime}\right)$ está relacionado com a quantidade de ruído das quadraturas amplitude e fase do campo incidente $S_{p}\left(\nu^{\prime}\right)$ e $S_{q}\left(\nu^{\prime}\right)$.

Para obter a expressão acima foram consideradas nulas as correlações entre as quadraturas amplitude e fase do feixe incidente, $C_{q p}(\nu)=0$, dadas por

$$
\delta\left(\nu^{\prime}-\nu^{\prime \prime}\right) C_{q p}=\left\langle\delta p_{i n}\left(\nu^{\prime}\right) \delta q_{i n}\left(-\nu^{\prime \prime}\right)\right\rangle
$$

A correlação $C_{q p}$ nos dá uma medida de quão dependentes são essas variáveis. Variáveis independentes satisfazem $C_{q p}=0$. Esse caso ocorre quando os eixos menor e maior da elipse de ruído estão completamente alinhados às quadraturas $p$ e $q$, tal como apresentado na figura 2.3.

Podemos agora, analisar a equação do espectro de ruído para diferentes valores de frequências de análise ou, se quisermos, para diferentes modos de bandas laterais. Gráficos da função (2.7.40) usando os valores $S p=0,5$ e $S q=2,0$ estão ilustrados na figura 2.8. A curva tracejada em vermelho, referente a $\nu^{\prime}=4$, mostra de modo claro a conversão 


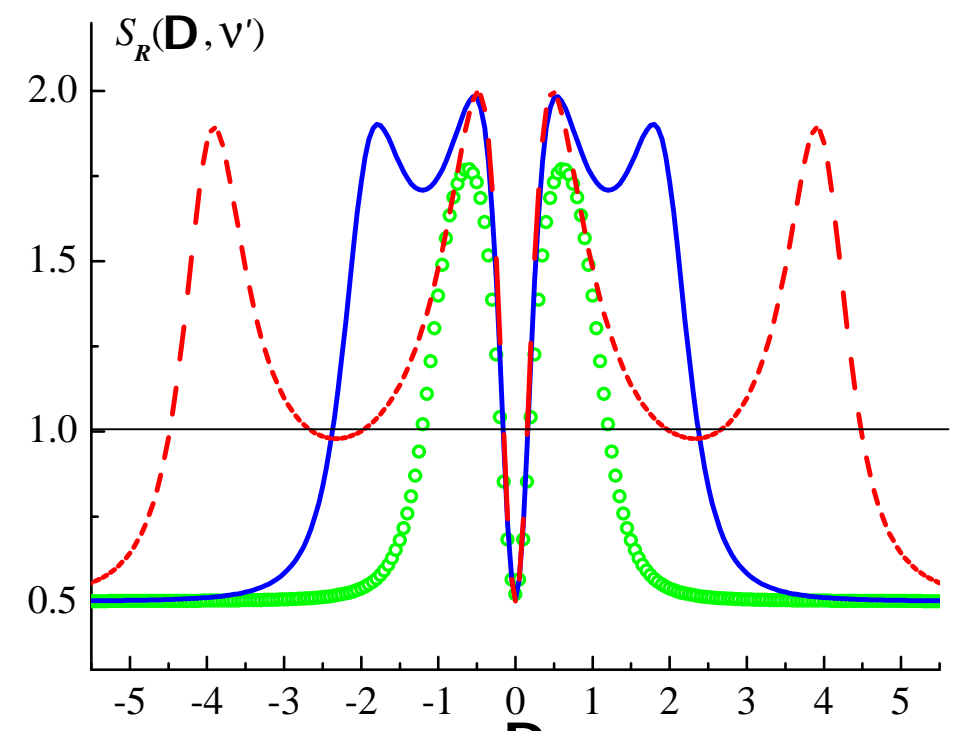

Figura 2.8: Curvas de ruído $S_{R}\left(\Delta, \nu^{\prime}\right)$ em função da dessintonia para diferentes valores de $\nu^{\prime}$. Círculos abertos verdes: $\nu^{\prime}=1$; Curva em azul: $\nu^{\prime}=2$; Tracejada em vermelho: $\nu^{\prime}=4$. Foram utilizados $S_{p}\left(\nu^{\prime}\right)=0.5$ e $S_{p}\left(\nu^{\prime}\right)=2.0$.

de fase em amplitude para quatro valores de dessintonia, dois nos extremos $\Delta= \pm 4$, correspondentes à passagem pela ressonância com as bandas laterais positiva e negativa, e dois centrais $\Delta= \pm 0,5$, referentes à mudança de fase da portadora central. Neste caso, as rotações da elipse de ruído ocorrem quase de maneira independente. Para $\nu^{\prime}=2$, curva contínua em azul, continuamos a ver a conversão quando a ressonância da cavidade se aproxima da frequência das bandas laterais. Porém, os quatro picos aparecem bem próximos uns dos outros, o que leva crer que a cavidade já não consegue resolver efetivamente as três frequências. Nesta situação, há uma região de interferência entre as fases das bandas laterais e da portadora central. Mesmo nessas condições, é possível quantificar com precisão o valor do ruído de fase do campo.

Vê-se isso mais claramente calculando o valor mínimo da frequência de análise que propicia o giro completo da elipse de ruído. Isto pode ser feito impondo a condição $\theta_{R}(\Delta)-\theta_{R}\left(\Delta-\nu^{\prime}\right)=\pi / 2$, retirada da equação (2.7.38), de onde é possível mostrar que a condição a ser satisfeita é $\nu^{\prime}>\sqrt{2}$ [Galatola 1991, Villar 2007b]. Quando $\nu^{\prime}$ não satisfaz a condição mínima (por exemplo, $\nu^{\prime}=1$ ) não há distinção entre as bandas laterais e a portadora, conforme apresentado pelos círculos verdes, o que implica um giro parcial da elipse de ruído, algo que gera valores subestimados para a potência do ruído de fase. Os resultados experimentais apresentados nesta tese possuem características similares às encontradas na curva de cor azul.

Em nosso experimento, existe o interesse pela medida conjunta de quadraturas de três campos distintos. Fazemos isso ajustando seus parâmetros de tal maneira que as 
elipses de ruído dos campos rotacionam de forma síncrona, algo realizado pela variação simultânea das dessintonias das cavidades de análise. Desta forma, além de acessar os ruídos de amplitude e fase de cada campo de forma independente, conseguimos ter acesso às correlações entre quadraturas do mesmo tipo, i.e. $C\left(\delta \hat{p}_{i} \delta \hat{p}_{j}\right)$ e $C\left(\delta \hat{q}_{i} \delta \hat{q}_{j}\right)$, para campos distintos, identificados pelos subíndices $i$ e $j$.

Esses valores podem ser obtidos calculando a correlação simetrizada a partir da expressão

$$
C\left(\delta \hat{p}_{R_{1}} \delta \hat{p}_{R_{2}}\right)=\frac{1}{2}\left\langle\delta p_{R_{1}}\left(\nu^{\prime}\right) \delta p_{R_{2}}\left(-\nu^{\prime}\right)+\delta p_{R_{1}}\left(-\nu^{\prime}\right) \delta p_{R_{2}}\left(\nu^{\prime}\right)\right\rangle
$$

$\operatorname{com} \delta p_{R_{i}}\left( \pm \nu^{\prime}\right)(i=1,2)$ dados pela expressão (2.7.38). Desenvolvendo a equação acima, obtemos explicitamente

$$
\begin{aligned}
C\left(\delta \hat{p}_{R_{1}} \delta \hat{p}_{R_{2}}\right) & =\operatorname{Re}\left[g_{p}^{(1)}\left(\nu^{\prime}\right) g_{p}^{(2) *}\left(\nu^{\prime}\right)\right] C\left(\hat{p}_{1} \hat{p}_{2}\right)+\operatorname{Re}\left[g_{q}^{(1)}\left(\nu^{\prime}\right) g_{q}^{(2) *}\left(\nu^{\prime}\right)\right] C\left(\hat{q}_{1} \hat{q}_{2}\right) \\
& +\operatorname{Re}\left[i g_{p}^{(1)}\left(\nu^{\prime}\right) g_{q}^{(2) *}\left(\nu^{\prime}\right)\right] C\left(\hat{p}_{1} \hat{q}_{2}\right)+\operatorname{Re}\left[i g_{q}^{(1)}\left(\nu^{\prime}\right) g_{p}^{(2) *}\left(\nu^{\prime}\right)\right] C\left(\hat{q}_{1} \hat{p}_{2}\right)
\end{aligned}
$$

Para ter acesso à correlações cruzadas, i.e. $C\left(\delta \hat{p}_{i} \delta \hat{q}_{j}\right)$ e $C\left(\delta \hat{q}_{i} \delta \hat{p}_{j}\right)$, utilizamos um artifício simples: variamos a dessintonia da cavidade de um dos campos e deixamos a outra cavidade completamente fora de ressonância. No primeiro caso, já sabemos que haverá alternância entre flutuações de quadraturas, no segundo somente a quadratura amplitude será medida. Desse modo, a medida das flutuações de fase de um campo será feita no mesmo instante em que medimos as flutuações da quadratura amplitude do outro, permitindo quantificar o valor da correlação $C\left(\delta \hat{q}_{i} \delta \hat{p}_{j}\right)$. Quando mais de dois campos são analisados, é necessário repetir esse processo para todos os pares possíveis, a fim de medir todos os termos da matriz de covariâncias do sistema. No capítulo referente aos resultados experimentais, será dado um exemplo prático de reconstrução do estado de três campos de luz intensos.

Esta é a técnica que vem sendo amplamente utilizada em nosso grupo e atualmente tem se revelado mais completa que a detecção homodina usual, em medidas no domínio da frequência [Barbosa 2012]. Este é um assunto que tratamos de forma breve aqui, mencionando aquilo que é de interesse direto para os estados de interesse dessa tese. Mais detalhes poderão ser encontrados na tese de outro estudante de doutorado do grupo, Felippe A. S. Barbosa. Para um estudo minucioso sobre a técnica auto-homodina recomendamos as referências [Villar 2007b, Villar 2008]. No próximo capítulo falaremos sobre o fenômeno de interesse, o emaranhamento, e descreveremos os critérios que permitem identificá-lo em nosso sistema.

Apresentamos neste capítulo conceitos que servem de base para os capítulos seguintes. Estes conceitos são fundamentais para o entendimento do Oscilador Paramétrico Ótico e 
análise dos dados apresentados no capítulo de resultados experimentais. Descrevemos de forma breve o campo eletromagnético no contexto clássico e quântico. Foram definidas as quadraturas amplitude e fase, bem como as flutuações a elas associadas, organizadas posteriormente em uma matriz de covariâncias. Detalhamos o processo de fotodetecção do campo, explicitando quais as frequências efetivamente envolvidas no processo. Definimos o shot-noise, a referência para os ruídos do campo. E, finalmente, discutimos as técnicas de detecção homodina e auto-homodina, indicando quais informações podem ser extraídas por cada técnica. 


\section{Capítulo 3}

\section{Emaranhamento}

Pois se foi permitido ao Homem

Tantas coisas conhecer

É melhor que todos saibam

$O$ que pode acontecer.

G. Gil (1976)

Este capítulo tem como objetivo apresentar as ideias iniciais acerca do emaranhamento, fenômeno intrinsecamente quântico e que nos dias atuais tem despertado bastante interesse principalmente por sua aplicação no processamento e transmissão de informação quântica [Nielsen 2000]. Contudo, em primeiro lugar, à parte todo o apelo tecnológico que impulsiona investimentos e motiva a pesquisa nos dias atuais, o emaranhamento é por si só um fenômeno interessante do ponto de vista de ciência básica, revelando propriedades fundamentais de uma teoria que continua revolucionando nosso mundo.

A seguir, serão expostas as primeiras ideias e reflexões que deram origem ao emaranhamento, situações na época consideradas absurdas e inimagináveis na prática, colocadas como aspectos frágeis da mecânica quântica e que atualmente são realizadas experimentalmente, dando ainda mais força à teoria. Três excelentes referências nas quais foram baseadas parte deste capítulo são fortemente recomendadas para ajudar a compreender o desenvolvimento histórico e filosófico do emaranhamento [Isaacson 2008, Jagdish 2001, Stanford WebPage].

Em seguida, será dada a definição formal de estados emaranhados baseada nas referências [Piza 2009, Rigolin 2005], cuja leitura é fortemente recomendada, dada a apresentação didática que não se perde em meio a formalismos matemáticos. Ainda neste contexto, também é aconselhada com veemência a leitura dos trabalhos [Villar 2007b, Aolita 2008].

Por fim, serão apresentados os critérios utilizados na análise dos estados de interesse dessa tese. Os critérios são: soma de variâncias proposto por Duan et al. [Duan 2000] para um sistema bipartite e o critério de positividade sob transposição parcial (PPT), idealizado por Asher Peres [Peres 1996] no âmbito de variáveis discretas e estendido para 
variáveis contínuas por Simon [Simon 2000]. Tais critérios são de extrema utilidade na análise feita nesta tese por apresentarem características bastante importantes quando os estados analisados são gaussianos, pois têm a qualidade de serem necessários e suficientes para tais estados. O tratamento detalhado sobre identificação, caracterização e quantificação de emaranhamento em estados gaussianos é encontrado na referência [Adesso 2006]. Apresentaremos aqui somente os resultados que são de relevância direta para o desenvolvimento dessa tese.

\subsection{Primeiras reflexões}

Muito se sabe sobre Mecânica Quântica atualmente. Trata-se de uma teoria com uma estrutura matemática bem compreendida, em ótimo acordo com os resultados experimentais. No entanto, apesar de descrever vários fenômenos previamente inexplicáveis, muitas vezes nos lança em situações completamente contra-intuitivas, longe de serem óbvias. De certa forma, o uso habitual da teoria quântica e a observação da sua eficácia faz-nos tratar como obviedade algumas de suas características fundamentais como, por exemplo, o princípio da incerteza de Heisenberg, que proíbe determinar simultaneamente e com precisão absoluta os valores de duas grandezas ditas conjugadas, tais como posição e momento de uma partícula, e o mecanismo obscuro da redução do pacote de onda.

Eram justo estas características da Mecânica Quântica que fomentavam em Einstein a ideia de que algo estava faltando na teoria. Segundo ele, as grandezas físicas deveriam ser dotadas de "realismo", uma situação real que existe independente de observações, e não se tornarem reais somente quando observadas, de acordo com as regras quânticas. Este desconforto aumentava com o passar dos anos, levando-o a conjurar problemas que, seguindo as regras da teoria quântica, violavam princípios fundamentais da visão clássica do mundo. Em 1933, ao final de um seminário ministrado por Léon Rosenfeld sobre a mensurabilidade de grandezas do campo eletromagnético, Einstein relata um experimento mental (Gedankenexperiment) bastante famoso atualmente:

What would you say about the following situation? Suppose two particles are set in motion towards each other with the same, very large momentum and that they interact with each other for a very short time when they pass at known positions. Consider now an observer who gets hold of one of the particles, far away from the region of interaction, and measures its momentum; then, from the conditions of the experiment, he will obviously be able to deduce the momentum of the other particle. If, however, he chooses to measure the position of the first particle, he will be able to tell where the particle is. This is a perfectly correct and straightforward deduction from the principles of quantum mechanics. 
Posto dessa forma, o problema levantado por Einstein traz consigo um questionamento sobre outro conceito importante, a "localidade": se duas partículas estiverem espacialmente distantes uma da outra, de modo a não haver mais interação, qualquer perturbação sobre uma partícula terá efeitos somente sobre esta, sendo completamente independente do que ocorre na outra. Assumindo como verdadeiras as premissas da teoria quântica, essa definição vai de encontro ao experimento proposto no qual a posição (momento) de uma partícula passa a ser completamente determinada pela medida da posição (momento) da segunda, sem que estejam em uma região de interação. De um modo mais dramático, como os valores de posição e momento não podem ser acessados de modo concomitante, a realidade das duas grandezas parece estar condicionada à escolha da medida.

Para Einstein, a física deveria ser munida de realismo e localidade, livre de uma ação "fantasmagórica à distância". Contudo, apesar de a localidade ser algo completamente razoável do ponto de vista clássico, era somente uma suposição que nunca tinha sido provada.

Após refletirem mais sobre o Gedankenexperiment, Einstein, Nathan Rosen e Boris Podolsky publicaram em maio de 1935 um artigo de quatro páginas conhecido, pelas iniciais dos seus autores, como EPR [Einstein 1935]. O título "Can Quantum Mechanical Description of Physical Reality Be Considered Complete?" já deixava clara a crítica à Mecânica Quântica. Trata-se do artigo mais citado de Einstein, com mais de 10400 citações atualmente que, mesmo tendo gerado grande repercussão, foi publicado em uma versão que não o agradava completamente, levando-o a fazer queixas com Schrödinger, pois segundo Einstein a clareza conceitual do problema teria sido comprometida pela quantidade de formalismo matemático inserido por Podolsky.

No artigo, o ponto de partida dos autores é a definição de "elemento de realidade":

If without in any way disturbing a system we can predict with certainty the value of a physical quantity, then there exists an element of physical reality corresponding to this physical quantity.

[Einstein 1935]

Assim, caso seja possível dizer qual a posição de uma dada partícula com absoluta certeza sem a necessidade de perturbá-la, dizemos então que a posição da partícula é real e existe independentemente de observações. Esta é a base para o experimento mental proposto por Einstein, pois uma vez medida a posição ou momento da primeira partícula, torna-se possível determinar a posição ou momento da segunda sem perturbá-la. Portanto, essas grandezas seriam reais, segundo sua definição, e o acesso aos seus valores proibido por lacunas existentes na teoria quântica, sendo negativa a resposta para a pergunta feita no título do artigo.

Ao tomar conhecimento do artigo EPR, Bohr passou a pensar no problema procurando 
formas de argumentar em favor da teoria quântica. Em outubro de 1935, um artigo de Bohr com mesmo título foi publicado em resposta aos argumentos EPR [Bohr 1935]. Trata-se de um texto mais hermético e de difícil compreensão, no qual Bohr admite que no experimento proposto por Einstein realmente não há qualquer perturbação no segundo sistema. Para Bohr, a origem do princípio da incerteza estava relacionada com o distúrbio causado pelo ato da medida, o que de certa forma era colocado em xeque na argumentação EPR, daí então chamado de "paradoxo EPR".

Contudo Bohr argumenta que as duas partículas são na realidade partes de um todo, pois após o processo de interação passam a ter suas identidades superpostas de tal modo que a descrição do sistema global é dada por uma única função de onda. Além disso, Bohr ainda defendia que no argumento de Einstein não há qualquer exclusão do princípio da Incerteza de Heisenberg, que afirma não ser possível saber precisamente os valores da posição e momento simultaneamente.

De fato, no problema EPR, se medirmos a posição da partícula A saberemos certamente a posição da partícula distante B; da mesma forma, caso seja medido o momento da partícula A, o momento da partícula B será bem determinado. Isso é completamente razoável do ponto de vista quântico, visto que posições relativas e momento total são grandezas compatíveis (comutam). Contudo, em nenhum momento é dito que de fato é possível saber em um mesmo instante esses dois atributos da partícula $\mathrm{A}$, o que não tornam conhecidos os mesmos atributos da partícula $\mathrm{B}$, não havendo qualquer conflito com o princípio da incerteza. Essa situação nos remete a uma famosa citação de Asher Peres: "Unperformed experiments have no results" [Peres 1978]. Mesmo assim, a questão sobre a localidade ainda despertava bastante discussão, pois era tratada por Einstein como algo fundamental na natureza, uma vez que nenhum sinal poderia ser transmitido de uma partícula para outra com uma velocidade superior à da luz.

Dado o desconforto ainda existente, novos experimentos começaram a ser idealizados, dessa vez com Erwin Schrödinger, que, tendo uma contribuição enorme no desenvolvimento da mecânica quântica, manifestava grande interesse nos experimentos propostos por Einstein, principalmente por questões envolvendo a localidade. Para Schrödinger a localidade não era algo inviolável, pois poderiam existir correlações entre partículas espacialmente distantes que interagiram anteriormente. A fim de descrever essa característica, Schrödinger usou o termo emaranhamento (do alemão, Verschränkung):

Entanglement of predictions arises from the fact that two bodies at some earlier time formed in a true sense one system, that is were interacting, and have left behind traces on each other. If two separated bodies enter a situation in which they influence each other, and separate again, then there occurs what I have just called entanglement of our knowledge of the two bodies.

[Schrödinger 1935, English translation by John D. Trimmer (1980)] 
O trecho acima foi retirado da tradução do famoso artigo de Schrödinger, inspirado no artigo EPR, no qual ele apresenta o paradoxo do "Gato de Schrödinger". Este problema surge como uma tentativa de mostrar que a teoria quântica leva a situações absurdas, como a de uma superposição de um gato vivo e morto, sem que seja necessário recorrer a argumentos envolvendo localidade. Neste mesmo artigo, Schrödinger aponta que a característica essencial para a existência de estados que apresentam emaranhamento é o princípio da superposição das funções de onda de partículas que interagiram em algum momento.

Seguindo a apresentação dada em [Raymond 2008], veremos a seguir as visões de EPR e Schrödinger a respeito do problema lançado em 1933 por Einstein.

\subsection{Estados de Einstein, Podolsky e Rosen}

Einstein, Podolsky e Rosen consideraram duas partículas, A e B, que interagem durante um dado intervalo de tempo e em seguida se separam de modo a não haver mais interação. O sistema formado pelas duas partículas é preparado de tal modo que são bem definidos os valores da posição relativa $\left(\hat{x}=\hat{x}_{A}-\hat{x}_{B}\right)$ e momento total $\left(\hat{p}=\hat{p}_{A}+\hat{p}_{B}\right)$ com valores bem definidos $\mathbf{x}_{\mathbf{0}}$ e $\mathbf{0}$, respectivamente, algo possível por conta da compatibilidade dessas duas grandezas, conforme chamou a atenção Bohr no seu artigo em resposta a EPR. O estado inicial particular sugerido por EPR é descrito por uma função envolvendo o sistema global composto pelas duas partículas,

$$
\Psi_{E P R}\left(x_{A}, x_{B}\right)=\int_{-\infty}^{+\infty} \frac{d k}{2 \pi} F(k) e^{i k\left(x_{A}-x_{B}+x_{0}\right)} .
$$

Uma função desta forma é um autoestado do operador $\hat{p}=\hat{p}_{A}+\hat{p}_{B}$ com autovalor nulo

$$
\left(\hat{p}_{A}+\hat{p}_{B}\right) \Psi_{E P R}\left(x_{A}, x_{B}\right)=0 .
$$

Quando consideramos a medida de um dos momentos, $\hat{p}_{A}$ por exemplo, algo interessante ocorre com o estado. De acordo com os postulados da teoria quântica, se obtivermos como resultado da medida de $\hat{p}_{A}$ o autovalor $\hbar k_{0}$, a função de onda resultante será um autoestado associado a este autovalor,

$$
\begin{array}{r}
\hat{p}_{A} \Psi_{\mathrm{EPR}}\left(x_{A}, x_{B}\right)=\frac{\hbar}{2 \pi} \int_{-\infty}^{+\infty} F(k) k e^{i k\left(x_{A}-x_{B}-x_{0}\right)} d k \\
\hbar k_{0} \longrightarrow \Psi_{\text {red }}\left(x_{A}, x_{B}\right) \propto F\left(k_{0}\right) e^{i k_{0}\left(x_{A}-x_{B}-x_{0}\right)} .
\end{array}
$$

A função de onda resultante $\Psi_{\text {red }}\left(x_{A}, x_{B}\right)$ é um autoestado do operador $\hat{p}_{B}$ com autovalor $-\hbar k_{0}$. A implicação disso é que, em qualquer instante de tempo, ao medir o momento da partícula $B$ o resultado obtido será $-\hbar k_{0}$, pois $\hat{p}_{A}$ e $\hat{p}_{B}$ são constantes de movimento no caso de partículas livres de interação. Assim, a medida de $\hat{p}_{A}$ determina 
completamente o valor de uma medida subsequente de $\hat{p}_{B}$. De acordo com a definição de Einstein, a grandeza $\hat{p}_{B}$ é um elemento de realidade, sendo o mesmo válido para a análise feita para os operadores de posição $\hat{x}_{A}$ e $\hat{x}_{B}$. Portanto, as duas grandezas teriam valores bem determinados, independente de observações.

No que diz respeito à localidade, podemos considerar que a partícula A teve seu momento medido no instante $t_{A}$ e a partícula $\mathrm{B}$ no instante $t_{B}$ de forma que a distância $L$ entre as partículas seja tal que $\left(t_{B}-t_{A}\right)<L / c$, onde $c$ é a velocidade da luz. Mesmo neste caso o valor de $\hat{p}_{B}$ é completamente determinado pela medida de $\hat{p}_{A}$. Foi este tipo de situação que Einstein chamou de ação fantasmagórica à distância. Como dito anteriormente, Schrödinger não considerava essa característica absurda, uma vez que admitia possibilidade de correlações entre partículas que interagiram em um instante anterior, dando o nome emaranhamento a essa característica. A questão que surgia é se todas as correlações existentes poderiam ser explicadas somente por mecanismos clássicos. Vejamos a seguir o conceito de Schrödinger.

\subsection{Estados emaranhados segundo Schrödinger}

A fim de entender o argumento de Schrödinger podemos pensar no caso em que o estado de duas partículas independentes é escrito como

$$
\phi\left(x_{A}, x_{B}\right)=\eta\left(x_{A}\right) \xi\left(x_{B}\right) .
$$

Esta situação é diferente da encontrada no problema EPR. A probabilidade conjunta de encontrar a partícula A dentro do intervalo $d x_{A}$ no entorno de $x_{A 0}$ e a partícula $\mathrm{B}$ no intervalo $d x_{B}$ no entorno de $x_{B 0}$ é dada pelo produto das probabilidades individuais

$$
d P\left(x_{A 0}, x_{B 0}\right)=\left|\eta\left(x_{A 0}\right)\right|^{2} d x_{A}\left|\xi\left(x_{B 0}\right)\right|^{2} d x_{B}
$$

sendo os resultados completamente descorrelacionados, análogos a resultados de cara ou coroa obtidos quando duas pessoas lançam, cada uma, uma moeda. O mesmo raciocínio pode ser aplicado para medidas dos momentos das partículas. Portanto, as medidas das grandezas da partícula A nada nos dizem sobre os valores das mesmas grandezas associadas à partícula $B$.

Classicamente, podemos pensar em duas partículas completamente descritas por suas posições e momentos, $\left(x_{A}, p_{A}\right)$ e $\left(x_{B}, p_{B}\right)$, de tal modo que o sistema global é representado em um espaço de fases quadridimensional: $\left(x_{A}, p_{A}, x_{B}, p_{B}\right)$. Na física clássica essas grandezas podem possuir valores simultaneamente bem definidos de modo que um ponto $\left(x_{A 0}, p_{A 0}, x_{B 0}, p_{B 0}\right)$ nos dá toda informação sobre o sistema em um dado instante, além de fornecer toda informação sobre os constituintes em seus respectivos espaços de fase individuais. 
Já no cenário quântico, não podemos atribuir a descrição do estado das partículas por meio de um ponto no espaço de fase, devido ao princípio da incerteza de Heisenberg. O sistema é descrito por uma função de onda que não necessariamente viola a noção clássica de separabilidade. Um exemplo disso é a função $\phi\left(x_{A}, x_{B}\right)$ que fornece máxima informação sobre o sistema composto de duas partículas, sendo cada uma descrita por uma função de onda específica. Contudo, o estado EPR $\Psi_{E P R}\left(x_{A}, x_{B}\right)$ mesmo fornecendo toda informação contida no sistema total não permite que os constituintes sejam descritos por funções de onda únicas, levando à conclusão de que o conhecimento do sistema global não leva à descrição de suas partes isoladamente, conforme dito pelo próprio Schrödinger [Schrödinger 1935]:

"Maximal knowledge of a total system does not necessarily include total knowledge of all its parts, not even when these are fully separated from each other and at the moment are not influencing each other at all."

[Schrödinger 1935, English translation by John D. Trimmer (1980)]

Para se ter maior clareza sobre o que realmente diferencia a quantidade informação contida no sistema global e a obtida pela soma das informações dos sistemas individuas, pode-se recorrer a uma situação experimental simples. Dois observadores, cada um responsável por realizar medidas em subsistema, obtêm sua própria estatística e, simplesmente somando as informações obtidas separadamente, acabam excluindo as correlações existentes entre os subsistemas, deixando a descrição do sistema global incompleta por causa desta exclusão.

As ideias de Einstein e Schrödinger, apesar de gerarem grandes discussões e dúvidas, eram difíceis de serem respondidas a menos que traduzidas para um contexto onde a experimentação pudesse ser aplicada. Com uma versão mais simples do estado EPR, Bohm motivou John Bell na criação de desigualdades baseadas em teorias realistas locais, que permitiam verificar experimentalmente qual das previsões seria a correta, se as destas teorias ou as da mecânica quântica. Dada sua simplicidade, apresentaremos o exemplo de Bohm e suas consequências.

\subsection{O estado de Bohm-Aharonov}

Em 1951, após inúmeras discussões sobre processos de medida na teoria quântica [Bohm 1951, Bohm 1952a, Bohm 1952b], David Bohm e Yariv Aharonov propuseram uma nova versão do sistema EPR, conceitualmente equivalente porém com uma dinâmica mais simples de ser tratada matematicamente. Trata-se de uma molécula composta por dois átomos de spin $1 / 2$, em uma configuração que torna nulo o spin total conjunto. Pode-se 
ainda mostrar que qualquer que seja a base utilizada para representar o sistema, as partes possuem spins opostos.

Bohm supõe que a molécula é desintegrada por algum processo que mantém nulo o spin total, de modo que os átomos se afastam até que não haja mais interação entre eles. Para que haja conservação, os átomos, mesmo quando afastados, devem ser representados por estados onde o spin de um seja exatamente oposto ao spin do outro, o que implica presença de correlações perfeitas. Portanto, o estado puro de dois spins mais geral possível para o sistema é do tipo

$$
|\Psi\rangle=\frac{1}{\sqrt{2}}(|\uparrow \downarrow\rangle-|\downarrow \uparrow\rangle)
$$

que por simplificação foi escrito na notação de Dirac, onde $|\uparrow \downarrow\rangle \equiv|\uparrow\rangle_{1} \otimes|\downarrow\rangle_{2}$ indica estado $|\uparrow\rangle$ (spin igual a $+\hbar / 2$ ) para o primeiro átomo e $|\downarrow\rangle$ (spin igual a $-\hbar / 2$ ) para o segundo.

Antes de analisar o efeito de medidas sobre o estado proposto por David Bohm, vamos considerar a seguinte situação. Uma fonte prepara pares de átomos de spin $1 / 2$, tal que a orientação do spin de cada átomo é fixada na direção $\vec{z}$ e pode ser $+1 / 2$ ou $-1 / 2$. A fonte determina os spins dos átomos de modo aleatório. Produzido o par, dois observadores $A$ e $B$ espacialmente separados recebem um átomo cada um e, de modo completamente independente, realizam medidas do spin, repetindo o procedimento para cada par gerado. Neste caso, os resultados das medidas de $A$ e $B$ serão também aleatórios, independente do operador de spin usado $\left(\hat{s}_{x}, \hat{s}_{y}\right.$ ou $\left.\hat{s}_{z}\right)$, sem haver qualquer tipo de correlação.

Agora, vamos supor que a fonte sempre produza pares de átomos com spins opostos enviando novamente um átomo para cada observador. Desse modo, sempre que o observador $A$ receber um átomo com spin $+1 / 2$ o observador $B$ receberá um com spin oposto, e vice-versa. Ao efetuarem suas medidas com o operador $\hat{s_{z}}$, por exemplo, os observadores perceberão uma aleatoriedade nos resultados, porém ao ser realizada a comparação dos mesmos notarão correlações perfeitas. Para os demais operadores, independente da orientação do spin, será sempre observada aleatoriedade nos resultados.

Por fim, considere uma fonte que produza o estado (3.4.1) proposto por Bohm. Após produzir este estado, a fonte envia um átomo para cada observador. Novamente, é realizada uma série de medidas para os três observáveis $s_{x}, s_{y}$ e $s_{z}$. Neste caso, o estado de cada átomo está maximamente indefinido, sendo obtida uma correlação perfeita somente quando se comparam medidas realizadas na mesma base. De um modo geral, qualquer que seja a direção do spin, dada pelo vetor unitário $\mathbf{u}$, o valor do observável $\left(\hat{\mathbf{s}}_{\mathbf{1}} \cdot \mathbf{u}\right) \otimes \hat{\mathrm{I}}_{2}$ é maximamente indefinido. Contudo, sempre que a medida deste observável produzir o resultado $\hbar m_{1}$ haverá uma completa determinação do valor de $\left(\hat{\mathrm{I}}_{1} \otimes \hat{\mathbf{s}}_{\mathbf{2}} \cdot \mathbf{u}\right)$, pois, como os spins são opostos, $\hbar m_{2}=-\hbar m_{1}$. Este tipo de correlação é impossível de ser realizada por meios clássicos, sendo uma característica da natureza quântica, fruto do princípio de superposição de estados. Esta é uma correlação análoga à encontrada no caso EPR. 
Em novembro de 1964, motivado pelo exemplo de Bohm, John Bell se juntou às discussões envolvendo o "paradoxo EPR". Bell analisou os resultados das medidas no estado de Bohm-Aharonov incluindo na análise um parâmetro $\lambda$, denotando uma simples variável ou um conjunto de variáveis, ou até mesmo um conjunto de funções, podendo assumir tanto valores discretos quanto contínuos [Bell 1964]. Este parâmetro teria a função de suprir as eventuais lacunas existentes na Mecânica Quântica, tornando-a uma teoria completa no sentido EPR.

Na formulação de 1964, Bell faz uso explícito da localidade, afirmando que as medidas realizadas em um determinado sistema não interferem em nada nos resultados obtidos pelo segundo, além de fazer considerações restritivas sobre a existência de (anti-)correlação perfeita no sistema, algo não necessário em sistemas emaranhados mais gerais. A análise deu origem à famosa desigualdade de Bell, que pode ser violada pela mecânica quântica mas tem que ser satisfeita dentro de um modelo realista local - os modelos de variáveis ocultas, caracterizadas pelo fator $\lambda$. A desigualdade de Bell foi colocada em um contexto experimental mais aplicável no ano de 1969 por Clauser, Horne, Shimony e Holt (CHSH) que formularam uma nova desigualdade com hipóteses menos restritivas [Clauser 1978], mais tarde (1971) rededuzida por Bell [Bell 1971].

A descrição didática e detalhada das desigualdades de Bell e CHSH pode ser encontrada nas referências [Piza 2009, Rigolin 2005]. Para uma descrição mais técnica e matematicamente mais formal, a referência [Barata 2002] é indicada.

As primeiras violações das desigualdades de Bell foram realizadas em 1972 por John F. Clauser e Stuart J. Freedman, analisando correlações entre polarizações lineares de fótons emitidos por uma cascata atômica de cálcio [Freedman 1972]. Contudo, a forma como os polarizadores eram ajustados no experimento não permitia eliminar completamente a classe de teorias locais, devido à presença de "loopholes de localidade". Estes se referem à possibilidade de que, no ato da medida, haja comunicação entre os subsistemas, através de sinais que viajam a velocidades menores que a da luz, o que daria origem às correlações [Greenberger 2009].

Outros experimentos motivados pela ideia de Clauser e Freedman vieram em seguida, mas sem sucesso. Somente no início da década de 1980 o caso foi resolvido por Alain Aspect e colaboradores em uma série de artigos [Aspect 1981, Aspect 1982, Aspect 1982b], cujos resultados estão em excelente acordo com as predições da mecânica quântica, indicando se tratar de fato de uma teoria que apresenta previsões distintas de uma teoria realista local. Além disso, resultados recentes mostram que teorias realistas não-locais também são incompatíveis com resultados obtidos para correlações quânticas existentes entre fótons emaranhados [Gröblacher 2007]. No entanto, para que sejam refutadas todas as hipóteses favoráveis às teorias de variáveis ocultas, ainda se faz necessário realizar experimentos que eliminem simultaneamente tanto o problema relacionado à eficiência dos detectores, que dão origem aos "loopholes de detecção", quanto a localidade das medidas. 
Após sua verificação experimental, a ideia de que o emaranhamento poderia ser quantificado começou a se difundir. Além disso, essa característica quântica passou a ganhar notoriedade como uma grandeza que talvez pudesse ser utilizada como recurso para diferentes tarefas no contexto de processamento de informação, pois comparando com os recursos clássicos disponíveis atualmente, o emaranhamento passou a ser visto como uma propriedade que permite maior performance na execução dessas tarefas [Nielsen 2000].

O emaranhamento é uma característica puramente quântica que ocorre no tratamento de sistemas compostos e, por conta disso, é primeiramente classificado de acordo com o número de constituintes do sistema. Além disso, dada sua importância como recurso para execução de tarefas no processamento de informação, é algo que precisa ser precisamente identificado. Nas seções seguintes será apresentada a definição formal de emaranhamento utilizando a notação de Dirac. Dois critérios utilizados ao longo desta tese serão tratados: critério de soma de variâncias (DGCZ)[Duan 2000] e positividade sob transposição parcial (PPT)[Peres 1996].

\subsection{Definição formal de estados emaranhados}

Emaranhamento é uma característica quântica que se manifesta em sistemas compostos, vistos como aqueles que são formados por dois (bipartite) ou mais (multipartite) subsistemas. Por simplicidade, vamos considerar um sistema formado por duas partículas, rotuladas pelas letras A e B [Piza 2009]:

i) Associados a esses sistemas temos dois espaços de Hilbert (em geral infinitos), $\mathscr{H}_{A}$ e $\mathscr{H}_{B}$, representados pelas bases ortonormais $\left\{|m\rangle_{A}\right\}$ e $\left\{|n\rangle_{B}\right\}$ (onde $n, m=1,2,3, \ldots$ ) munidos de produtos escalares hermiteanos.

ii) O espaço de Hilbert do sistema composto é dado pelo produto tensorial dos espaços de Hilbert das partículas $\left(\mathscr{H}=\mathscr{H}_{A} \otimes \mathscr{H}_{B}\right)$, construído a partir de todos os produtos $\left\{|m\rangle_{A} \otimes\right.$ $\left.|n\rangle_{B}\right\}$ possíveis. Os vetores do espaço-produto $\mathscr{H}$ são da forma

$$
|\Psi\rangle=\sum_{m, n} c_{m n}|m\rangle_{A} \otimes|n\rangle_{B} \equiv \sum_{m, n} c_{m n}|m n\rangle
$$

onde definimos uma notação mais compacta para o produto tensorial.

iii) Uma vez definidos os produtos escalares de $\mathscr{H}_{A}$ e $\mathscr{H}_{B}$, supondo a ortonormalidade das bases,

$$
\left\langle m n \mid m^{\prime} n^{\prime}\right\rangle \equiv\left\langle m \mid m^{\prime}\right\rangle\left\langle n \mid n^{\prime}\right\rangle=\delta_{m m^{\prime}} \delta_{n n^{\prime}}
$$

e dado um outro vetor $\left|\Psi^{\prime}\right\rangle=\sum_{m, n} c_{m n}^{\prime}\left|m_{A} n_{B}\right\rangle$ de $\mathscr{H}$, temos que

$$
\left\langle\Psi \mid \Psi^{\prime}\right\rangle=\sum_{m, n} c_{m n}^{*} c_{m n}^{\prime}=\left\langle\Psi^{\prime} \mid \Psi\right\rangle^{*},
$$


o que leva à condição de normalização $\sum\left|c_{m n}\right|^{2}=1$.

Desta forma, qualquer vetor $\left|\phi_{A}\right\rangle \otimes\left|\phi_{B}\right\rangle$ pertence ao espaço $\mathscr{H}$, com coeficientes $c_{m n}$ determinados a partir da expansão dos vetores $\left|\phi_{A(B)}\right\rangle$ em suas respectivas bases,

$$
\left|\phi_{A}\right\rangle=\sum_{m} \gamma_{m}^{A}|m\rangle_{A}, \quad\left|\phi_{B}\right\rangle=\sum_{n} \gamma_{n}^{B}|n\rangle_{B} \Longleftrightarrow\left|\phi_{A}\right\rangle \otimes\left|\phi_{B}\right\rangle=\sum_{m, n} \underbrace{\gamma_{m}^{A} \gamma_{n}^{B}}_{c_{m n}}|m n\rangle
$$

Logo, sempre que os coeficientes $c_{m n}$ puderem ser escritos como produtos $\gamma_{m}^{A} \gamma_{n}^{B}$, os vetores de $\mathscr{H}$ poderão ser fatorados. Porém, devido às diversas combinações lineares permitidas na construção de um vetor no espaço de Hilbert global, a fatoração é algo muitas vezes impossível. Exemplos simples das duas situações:

\begin{tabular}{cc} 
Estado fatorável & Estado não fatorável \\
\hline$|\Psi\rangle=\frac{1}{\sqrt{2}}(|00\rangle+|01\rangle)=\frac{1}{\sqrt{2}}|0\rangle \otimes(|0\rangle+|1\rangle)$ & $\left|\Phi^{ \pm}\right\rangle=\frac{1}{\sqrt{2}}(|00\rangle+|11\rangle)$ \\
$c_{00}=c_{01}=1 / \sqrt{2} ; c_{10}=c_{11}=0$ & $c_{00}=c_{11}=1 / \sqrt{2}$ \\
$\gamma_{m}^{A}=\delta_{0 m}$ e $\gamma_{n}^{B}=\left(\delta_{0 n}+\delta_{1 n}\right) / \sqrt{2}$ & $c_{10}=c_{01}=0$ \\
$c_{m n}=\gamma_{m}^{A} \gamma_{n}^{B}$ & $c_{m n} \nrightarrow \gamma_{m}^{A} \gamma_{n}^{B}$
\end{tabular}

Tabela 3.1: Exemplos de estados puros fatoráveis e maximamente emaranhados.

O exemplo acima pode ser entendido como um sistema composto de duas partículas de spin $1 / 2$, interpretando os estados $|0\rangle$ e $|1\rangle$ como $\left|+\frac{1}{2}\right\rangle$ e $\left|-\frac{1}{2}\right\rangle$, respectivamente. De modo especial, os estados $\left|\Phi^{ \pm}\right\rangle=\frac{1}{\sqrt{2}}(|00\rangle \pm|11\rangle)$ juntamente com $\left|\Psi^{ \pm}\right\rangle=\frac{1}{\sqrt{2}}(|01\rangle \pm|10\rangle)$ representam os exemplos mais simples de estados não fatoráveis e definem a amplamente conhecida base de Bell.

Estados de um sistema que não podem ser fatorados são chamados estados emaranhados. Esse raciocínio pode ser estendido para um número maior de subsistemas. No entanto, determinar se um estado pode ser fatorado ou não se torna um problema bem mais difícil. Podemos dizer que a definição de estados emaranhados ocorre de forma indireta, partindo de uma negação: um sistema é emaranhado quando não é separável.

Em um contexto mais geral, a definição de emaranhamento e separabilidade para estados puros e mistos é dada em termos das propriedades dos vetores de estado e da matriz densidade do sistema.

As definições a seguir, da forma como estão escritas, podem ser encontradas na referência [Rigolin 2005].

\section{Definição 1. Emaranhamento em Estados Puros}

Seja um sistema quântico composto de $N$ subsistemas tal que o espaço de Hilbert associado a ele é $H=\otimes_{j}^{N} H_{j}$, onde $H_{j}$ é o espaço de Hilbert associado a cada subsistema. Se $|\Psi\rangle \in H$ é o estado que descreve este sistema, então ele não está emaranhado se, e 
somente se, podemos escrevê-lo como $|\Psi\rangle=\otimes_{j}^{N}\left|\Psi_{j}\right\rangle$, onde $\left|\Psi_{j}\right\rangle \in H_{j}$.

Exemplos:

\begin{tabular}{cc} 
Estados Não-Emaranhados & Estados Emaranhados \\
\hline$|\Psi\rangle=|0\rangle \otimes|0\rangle$ & $\left|\Phi^{ \pm}\right\rangle=\frac{1}{\sqrt{2}}(|00\rangle \pm|11\rangle)$ \\
$|\Psi\rangle=\frac{1}{\sqrt{2}}(|00\rangle \pm|01\rangle)=\frac{1}{\sqrt{2}}|0\rangle \otimes(|0\rangle+|1\rangle)$ & $\left|\Psi^{ \pm}\right\rangle=\frac{1}{\sqrt{2}}(|01\rangle \pm|10\rangle)$
\end{tabular}

Definição 2. Emaranhamento em Estados Gerais

Seja um sistema quântico composto de $N$ subsistemas descrito por um operador densidade $\hat{\rho} \in \otimes_{j}^{N} A_{j}$, onde $A_{j}$ é o espaço de Hilbert formado por todos os operadores que atuam em $H_{j}$. Dizemos que $\hat{\rho}$ representa um sistema não emaranhado se, e somente se, ele pode ser escrito, para algum $k$, como uma soma convexa de produtos diretos:

$$
\hat{\rho}=\sum_{i=0}^{k} p_{i} \hat{\rho}_{i}^{j}=\sum_{i=0}^{k} p_{i}\left(\hat{\rho}_{i}^{1} \otimes \hat{\rho}_{i}^{2} \otimes \cdots \otimes \hat{\rho}_{i}^{N-1} \otimes \hat{\rho}_{i}^{N}\right)
$$

onde $p_{i} \geq 0, \sum_{i=0}^{k} p_{i}=1$ e $\hat{\rho}_{i}^{j} \in A_{j}$.

Exemplos:

\begin{tabular}{cc} 
Estados Não-Emaranhados & Estados Emaranhados \\
\hline$\hat{\rho}=\frac{1}{2}|00\rangle\left\langle 00\left|+\frac{1}{2}\right| 11\right\rangle\langle 11|$ & $\hat{\rho}=\frac{1}{3}\left|\Psi^{-}\right\rangle\left\langle\Psi^{-}\left|+\frac{2}{3}\right| \Phi^{-}\right\rangle\left\langle\Phi^{-}\right|$ \\
$\hat{\rho}=\frac{1}{2}|00\rangle\left\langle 00\left|+\frac{1}{2}\right| 01\right\rangle\left\langle 01\left|+\frac{1}{2}\right| 10\right\rangle\left\langle 10\left|+\frac{1}{2}\right| 11\right\rangle\langle 11|$ &
\end{tabular}

As definições 1 e 2 podem ser vistas como graus de independência dos subsistemas. A primeira se refere à fatorabilidade, enquanto a segunda está relacionada com a separabilidade. Em geral, um sistema separável não é necessariamente fatorável, o que implica a existência de correlações de origem clássica. Como exemplo, podemos considerar um sistema quântico bipartite descrito no espaço de Hilbert $\mathscr{H}=\mathscr{H}^{11} \otimes \mathscr{H}^{22}$, representado pelo operador

$$
\hat{\rho}_{\mathrm{sep}}=\sum_{j} p_{j} \hat{\rho}_{j}^{(1)} \otimes \hat{\rho}_{j}^{(2)}
$$

onde $p_{j}>0$ com $\sum_{j} p_{j}=1$ são probabilidades de origem clássica, e $\hat{\rho}_{j}^{(1)}$ e $\hat{\rho}_{j}^{(2)}$ operadores densidades que descrevem os subsistemas 1 e 2 , respectivamente. 
Para entender a origem de correlações clássicas, podemos supor que cada subsistema que compõe o estado global (3.5.5) está conectado a um dado dispositivo que produz em sua saída um estado $\hat{\rho}_{j}^{(i)}(i=1$ para o primeiro subsistema e $i=2$ para o segundo), condicionado à entrada de um número inteiro $j$. Assim, para obter o estado (3.5.5), basta conectar os dispositivos de mecanismos independentes a um gerador de números aleatórios que fornece a entrada $j$ com probabilidade $p_{j}$. A combinação desses três elementos nos leva a um estado separável, cujo valor esperado de um dado operador $\hat{A}=\hat{A}^{(1)} \otimes \hat{A}^{(2)}$ tem sua expressão dada por

$$
\langle\hat{A}\rangle=\left\langle\hat{A}^{(1)} \otimes \hat{A}^{(2)}\right\rangle=\operatorname{Tr}\left\{\hat{\rho}\left(\hat{A}^{(1)} \otimes \hat{A}^{(2)}\right)\right\}=\sum_{j} p_{j} \operatorname{Tr}\left\{\hat{\rho}_{j}^{(1)} \hat{A}^{(1)}\right\} \operatorname{Tr}\left\{\hat{\rho}_{j}^{(2)} \hat{A}^{(2)}\right\} .
$$

Assim, por conta do processo de geração do estado, toda correlação existente se dá no nível de probabilidades, proveniente somente do gerador de números aleatórios, que pode ter origem puramente clássica. Com um raciocínio semelhante, diz-se que o emaranhamento não pode ser gerado via operações locais com comunicação clássica $\left(\mathrm{LOCC}^{3}\right)$, onde operações locais são definidas como aquelas realizadas em cada subsistema separadamente e comunicação clássica faz referência a qualquer "canal clássico" de comunicação. Com este procedimento, dois observadores poderiam preparar uma série de estados seguindo um protocolo previamente estabelecido, de modo a obter um estado do tipo (3.5.5).

Para sistemas quânticos multipartite, o raciocínio do parágrafo anterior é essencialmente o mesmo, contudo questões mais difíceis de serem respondidas surgem no quesito estrutura de emaranhamento. A dificuldade reside em determinar como o emaranhamento está distribuído entre as partes, pois os subsistemas podem apresentar correlações quânticas de diferentes formas. Mesmo em casos "mais simples", nos quais os estados dos subsistemas são gaussianos, a estrutura de emaranhamento é difícil de ser determinada, podendo também apresentar uma dinâmica não-trivial.

No caso de um sistema tripartite, por exemplo, a primeira questão a ser analisada é se o estado global do sistema dado pelo operador densidade $\hat{\rho}$ pode ser escrito como uma soma convexa de termos bisseparáveis,

$$
\hat{\rho}=\lambda_{0} \sum_{j} p_{j}^{(0 \mid 12)} \hat{\rho}_{j}^{(0)} \otimes \hat{\rho}_{j}^{(1,2)}+\lambda_{1} \sum_{j} p_{j}^{(1 \mid 02)} \hat{\rho}_{j}^{(1)} \otimes \hat{\rho}_{j}^{(0,2)}+\lambda_{2} \sum_{j} p_{j}^{(2 \mid 01)} \hat{\rho}_{j}^{(2)} \otimes \hat{\rho}_{j}^{(0,1)},
$$

onde os termos $\lambda_{i}$ e $p_{j}^{(k \mid l m)}$ formam distribuições de probabilidades e os operadores $\hat{\rho}^{(i, j)}$ representam estados bipartites. Se o subsistema representado pelo operador densidade $\hat{\rho}^{(i, j)}$ for emaranhado, os estados representados pelo operador (3.5.7) são classificados como inseparáveis. Por outro lado, caso o sistema bipartite seja separável, $\hat{\rho}^{(j, k)}=\hat{\rho}^{(j)} \otimes \hat{\rho}^{(k)}$, a equação (3.5.7) se reduz a

$$
\hat{\rho}=\sum_{j} p_{j} \hat{\rho}_{j}^{(0)} \otimes \hat{\rho}_{j}^{(1)} \otimes \hat{\rho}_{j}^{(2)}
$$

\footnotetext{
${ }^{3}$ Acrônimo da expressão em inglês: Local Operations and Classical Communication.
} 
de onde se vê que o sistema tripartite é completamente separável.

Se for demonstrado que o estado geral do sistema não pode sequer ser escrito da forma (3.5.7), diz-se que o emaranhamento é do tipo genuíno, completamente inseparável com relação a qualquer tipo de partição.

Portanto, a equação (3.5.7) serve como indicadora das estruturas de emaranhamento tripartite possíveis, talvez um pouco mais fáceis de serem compreendidas por meio da representação pictórica da figura 3.1 .

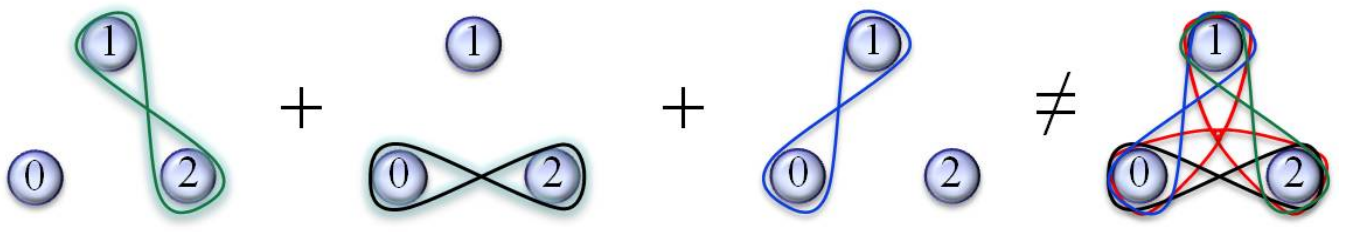

Figura 3.1: Representação pictórica de sistemas emaranhados e suas correspondências com a equação (3.5.7). Caso o sistema tripartite global possa ser escrito como uma soma de termos bisseparáveis, este sistema pode ser representado pela figura situada ao lado esquerdo do sinal de desigualdade. À direita, mostramos a figura de um estado emaranhado que elimina completamente sua representação por (3.5.7), o que exclui qualquer possibilidade de escrevê-lo como uma soma de estados separáveis por bipartições.

Apesar de relativamente simples de serem entendidas, as definições são negativas: um sistema é emaranhado se não é separável. Além disso, não são operacionais, pois não oferecem um procedimento ou critério capaz de decidir se há separabilidade ou não. Visto isso, percebe-se que a definição de separabilidade é simples, o grande problema passa a ser: dado um sistema quântico composto, como identificar, caracterizar e quantificar o emaranhamento deste sistema (caso exista)? É vasta a literatura que tem como objetivo responder esta pergunta, porém não se trata de uma tarefa simples, principalmente quando estão envolvidas perguntas sobre a estrutura do emaranhamento gerado.

A seguir discutiremos dois critérios que serão utilizados nesta tese: o critério de Duan et al. (também conhecido por critério da soma de variâncias ou DGCZ1) para o caso bipartite[Duan 2000] e o critério de positividade sob transposição parcial (PPT²) [Peres 1996]. O primeiro é bem mais simples de ser aplicado, pois faz necessária somente a medida dos momentos de segunda ordem (ou flutuações) de variáveis canonicamente conjugadas de cada subsistema. Já a aplicação do critério PPT demanda a reconstrução completa do estado realizada por meio da medida de todas as entradas da matriz de covariâncias.

Apesar desta dificuldade, a princípio técnica, o PPT gera bastante interesse por ser

\footnotetext{
${ }^{1}$ A denominação é dada pelo uso das iniciais dos últimos nomes de seus autores: Duan, Giedke, Cirac e Zoller.

${ }^{2}$ Acrônimo da expressão em inglês: Positivity under Partial Tranposition.
} 
um critério necessário e suficiente para estados gaussianos. Os estados discutidos nesta tese possuem uma estatística gaussiana.

\subsection{Soma de variâncias: caso bipartite}

O critério DGCZ, da forma como será tratado a seguir, segue de perto o desenvolvimento encontrado no artigo original [Duan 2000].

Consideremos os operadores de posição e momento $\hat{q}_{1}, \hat{q}_{2}, \hat{p}_{1}$ e $\hat{p}_{2}$ dos constituintes de um sistema bipartite separável representado pela matriz densidade (3.5.5). Os operadores de posição e momento obedecem a relação de comutação $\left[\hat{q}_{i}, \hat{p}_{j}\right]=i \delta_{i, j}$ e permitem definir novos operadores $\hat{u}$ e $\hat{v}$ dados por

$$
\hat{u}=\frac{\hat{q}_{1}-\hat{q}_{2}}{\sqrt{2}} \text { e } \hat{v}=\frac{\hat{p}_{1}+\hat{p}_{2}}{\sqrt{2}} .
$$

As variáveis $\hat{u}$ e $\hat{v}$ são conhecidas como variáveis do tipo EPR, pois são as mesmas mencionadas no problema por Einstein no artigo de 1935 [Einstein 1935]. Se não fizermos restrições sobre o operador densidade, é esperado que a soma das variâncias dos operadores $\hat{u}$ e $\hat{v}$ possua valor mínimo nulo, uma vez que variâncias são positivas. A pergunta que pode ser feita é se existe um valor mínimo diferente de zero para alguma classe de estados. Adiante, consideremos o sistema separável (3.5.5).

Para um dado operador densidade $\hat{\rho}$, após algumas manipulações algébricas simples, obtemos a variância do operador $\hat{u}$ :

$$
\begin{aligned}
\Delta_{\hat{\rho}}^{2} \hat{u} & =\left\langle\hat{u}^{2}\right\rangle_{\hat{\rho}}-\langle\hat{u}\rangle_{\hat{\rho}}^{2} \\
& =\sum_{i} \frac{p_{i}}{2}\left\langle\left(\hat{q}_{1}-\hat{q}_{2}\right)^{2}\right\rangle_{i}-\langle\hat{u}\rangle_{\hat{\rho}}^{2} \\
& =\sum_{i} \frac{p_{i}}{2}\left[\left\langle\hat{q}_{1}^{2}\right\rangle_{i}-2\left\langle\hat{q}_{1} \hat{q}_{2}\right\rangle_{i}+\left\langle\hat{q}_{2}^{2}\right\rangle_{i}\right]-\langle\hat{u}\rangle_{\hat{\rho}}^{2}
\end{aligned}
$$

com média $\langle\mathcal{O}\rangle_{\hat{\rho}}$ calculada em $\hat{\rho},\langle\mathcal{O}\rangle_{\hat{\rho}}=\sum_{i} \hat{p}_{i}\left\langle\Psi_{i}|\mathcal{O}| \Psi_{i}\right\rangle=\operatorname{Tr}\{\hat{\rho} \mathcal{O}\}$. A quantidade $\Delta_{\hat{\rho}}^{2} \hat{v}$ é calculada de forma análoga. Portanto, a soma das variâncias de $\hat{u}$ e $\hat{v}$ é dada pela expressão

$$
\begin{aligned}
\Delta_{\hat{\rho}}^{2} \hat{u}+\Delta_{\hat{\rho}}^{2} \hat{v} & =\sum_{i} \frac{p_{i}}{2}\left[\left\langle\hat{q}_{1}^{2}\right\rangle_{i}+\left\langle\hat{q}_{2}^{2}\right\rangle_{i}+\left\langle\hat{p}_{1}^{2}\right\rangle_{i}+\left\langle\hat{p}_{2}^{2}\right\rangle_{i}\right. \\
& \left.-2\left\langle\hat{q}_{1} \hat{q}_{2}\right\rangle_{i}+2\left\langle\hat{p}_{1} \hat{p}_{2}\right\rangle_{i}\right]-\langle\hat{u}\rangle_{\hat{\rho}}^{2}-\langle\hat{v}\rangle_{\hat{\rho}}^{2}
\end{aligned}
$$

Os cálculos feitos até aqui são válidos para qualquer operador densidade $\hat{\rho}$, pois nenhuma referência foi feita à separabilidade do sistema. A fim de explorar a separabilidade 
do estado (3.5.5), vamos reescrever a equação anterior como

$$
\begin{aligned}
\Delta_{\hat{\rho}}^{2} \hat{u}+\Delta_{\hat{\rho}}^{2} \hat{v} & =\frac{1}{2}\left(\Delta_{\hat{\rho}}^{2} \hat{q}_{1}+\Delta_{\hat{\rho}}^{2} \hat{q}_{2}+\Delta_{\hat{\rho}}^{2} \hat{p}_{1}+\Delta_{\hat{\rho}}^{2} \hat{p}_{2}\right) \\
& -\sum_{i} p_{i}\left[\left\langle\hat{q}_{1} \hat{q}_{2}\right\rangle_{i}-\left\langle\hat{q}_{1}\right\rangle_{i}\left\langle\hat{q}_{2}\right\rangle_{i}+\left\langle\hat{p}_{1} \hat{p}_{2}\right\rangle_{i}-\left\langle\hat{p}_{1}\right\rangle_{i}\left\langle\hat{p}_{2}\right\rangle_{i}\right] \\
& +\sum_{i} p_{i}\langle\hat{u}\rangle_{i}^{2}-\left[\sum_{i} p_{i}\langle\hat{u}\rangle_{i}\right]^{2}+\sum_{i} p_{i}\langle\hat{v}\rangle_{i}^{2}-\left[\sum_{i} p_{i}\langle\hat{v}\rangle_{i}\right]^{2}
\end{aligned}
$$

Para chegar à expressão anterior, foram adicionados zeros (i.e. $\sum_{i} p_{i}\left\langle\hat{q}_{1}\right\rangle_{i}^{2} / 2-\sum_{i} p_{i}\left\langle\hat{q}_{1}\right\rangle_{i}^{2} / 2$ ), de modo a obter as variâncias das quadraturas $\hat{p}_{i}$ e $\hat{q}_{i}$ e os termos entre colchetes na última linha.

De acordo com a equação (3.5.6), a separabilidade do sistema implica as igualdades $\left\langle\hat{q}_{1} \hat{q}_{2}\right\rangle_{i}=\left\langle\hat{q}_{1}\right\rangle_{i}\left\langle\hat{q}_{2}\right\rangle_{i}$ e $\left\langle\hat{p}_{1} \hat{p}_{2}\right\rangle_{i}=\left\langle\hat{p}_{1}\right\rangle_{i}\left\langle\hat{p}_{2}\right\rangle_{i}$, resultado que anula a segunda linha da equação precedente. Além disso, mais duas considerações são importantes nesta etapa:

i) o princípio da incerteza $\Delta^{2} \hat{q}_{j} \Delta^{2} \hat{p}_{j} \geq \frac{1}{4}\left|\left[\hat{q}_{j}, \hat{p}_{j}\right]\right|^{2}$ impõe um limite inferior para a soma das variâncias de $\hat{q}$ e $\hat{p}, \Delta^{2} \hat{q}_{j}+\Delta^{2} \hat{p}_{j} \geq\left|\left[\hat{q}_{j}, \hat{p}_{j}\right]\right| ;$

ii) a desigualdade de Cauchy-Schwarz, $\left(\sum_{i} p_{i}\right)\left(\sum_{i} p_{i}\langle\hat{u}\rangle_{i}^{2}\right) \geq\left(\sum_{i} p_{i}\left|\langle\hat{u}\rangle_{i}\right|\right)^{2}$, mostra que a última linha da equação (3.6.4) possui limite inferior nulo.

Com estes resultados, concluímos que todo sistema bipartite descrito pelo operador densidade separável (3.5.5) deve obedecer à desigualdade

$$
\Delta_{\text {sep }}^{2} \hat{u}+\Delta_{\text {sep }}^{2} \hat{v} \geq 2
$$

Esta desigualdade também é conhecida como critério de Duan [Duan 2000], cuja violação é suficiente para caracterizar a existência de emaranhamento em um sistema. O termo suficiente nos diz que existem estados emaranhados que não violarão a desigualdade, ou seja, o critério não representa uma solução definitiva para decidir se há emaranhamento ou não. Contudo, conforme demonstrado por Duan et al., a desigualdade (3.6.5) se torna um critério de inseparabilidade necessário e suficiente para estados gaussianos, desde que a matriz de covariâncias do sistema seja primeiramente disposta em uma forma denominada forma padrão do tipo II [Duan 2000], tornando-se equivalente ao critério de positividade sob transposição parcial [Peres 1996] estendido para sistemas descritos por variáveis contínuas [Simon 2000].

\subsection{Critério de Positividade sob Transposição Parcial (PPT)}

Aqui apresentamos o critério de positividade sob transposição parcial (PPT) elaborado por Peres [Peres 1996] para sistemas quânticos discretos compostos por dois subsistemas e a extensão do mesmo para variáveis contínuas realizada por Simon [Simon 2000]. 
Para sistemas bipartites, não é difícil encontrar critérios operacionais suficientes para identificar emaranhamento. No entanto, critérios suficientes que satisfaçam a condição de necessários são obtidos somente para alguns estados quânticos específicos, conforme mencionado na seção anterior para a soma de variâncias.

O critério PPT, também conhecido como critério de Peres-Horodecki [Horodecki M. 1996], torna-se necessário e suficiente somente para algumas classes de estados mais restritas. Será dada a seguir uma ideia geral do desenvolvimento e aplicação deste critério.

\subsubsection{Característica geral}

O critério se baseia na aplicação de mapas positivos: $\Lambda$ é um mapa positivo se sua aplicação em operadores $\hat{\rho}$ não-negativos de traço finito dá origem a operadores $\Lambda(\hat{\rho})$ também não-negativos de traço finito. Formalmente,

$$
\begin{aligned}
\hat{\rho} \quad: & \operatorname{Tr}[\hat{\rho}]<\infty ; \quad\langle\xi|\hat{\rho}| \xi\rangle \geq 0 \text { para todo }|\xi\rangle \text { de } \mathcal{H}, \quad\langle\xi \mid \xi\rangle=1 \\
& \Downarrow \\
\Lambda(\hat{\rho}) \quad: & \operatorname{Tr}[\Lambda(\hat{\rho})]<\infty ; \quad\langle\xi|\Lambda(\hat{\rho})| \xi\rangle \geq 0 \text { para todo }|\xi\rangle \text { de } \mathcal{H}, \quad\langle\xi \mid \xi\rangle=1 .
\end{aligned}
$$

Um mapa positivo comumente usado, dada sua operacionalidade, é o operador transposição $\mathcal{T}$. Isso significa que ao aplicarmos $\mathcal{T}$ em um operador positivo semi-definido $\mathcal{O}$, temos como resultado um operador $\mathcal{O}^{\prime}=\mathcal{T} \mathcal{O}$ também positivo semi-definido. Para mostrar que isto de fato ocorre, vamos seguir os mesmos passos da referência [Rigolin 2005].

A operação de transposição implica troca dos índices dos elementos de matriz, ou seja, $\mathcal{T} \mathcal{O}_{i j}=\mathcal{O}_{j i}$. Vamos considerar a transformação $\mathcal{U}$ que diagonaliza $\mathcal{O}$.

Como $\mathcal{O} \geq 0$ por definição, o operador diagonal $\mathcal{O}^{\prime}=\mathcal{U} \mathcal{O U}^{\dagger}$ é positivo semi-definido. Ao aplicarmos $\mathcal{T}$ em $\mathcal{O}^{\prime}$ temos ainda $\mathcal{T} \mathcal{O}^{\prime} \geq 0$, pois $\mathcal{O}^{\prime}$ só possui elementos na diagonal principal. Isso implica $\left.\mathcal{T} \mathcal{O}^{\prime}=\mathcal{T}\left(\mathcal{U} \mathcal{U}^{\dagger}\right)=\mathcal{T}\left(\mathcal{U}^{\dagger}\right) \mathcal{T O} \mathcal{T}(\mathcal{U})\right)^{3}$. Se definirmos $\mathcal{V}=\mathcal{T}\left(\mathcal{U}^{\dagger}\right)$ e $\mathcal{V}^{\dagger}=\mathcal{T}(\mathcal{U})$, temos $\mathcal{T} \mathcal{O}^{\prime}=\mathcal{V} \mathcal{T} \mathcal{O V}^{\dagger} \geq 0$. Mas $\mathcal{V} \mathcal{V}^{\dagger}=\mathcal{T}\left(\mathcal{U}^{\dagger}\right) \mathcal{T}(\mathcal{U})=\mathcal{T}\left(\mathcal{U} \mathcal{U}^{\dagger}\right)=\mathcal{I}$, onde $\mathcal{I}$ é uma matriz identidade. Então $\mathcal{V}$ é uma transformação unitária. Como transformações unitárias não mudam os autovalores de um operador e $\mathcal{V} \mathcal{T} \mathcal{O V}^{\dagger} \geq 0$, consequentemente $\mathcal{T O} \geq 0$. Concluímos, então, que $\mathcal{T}$ é um mapa positivo. Vejamos agora como podemos aplicar o operador transposição como um teste de separabilidade de estados quânticos.

\subsubsection{Variáveis discretas}

Partindo de propriedades de matrizes densidade [Cohen-Tannoudji 1977] e do mapa positivo $\mathcal{T}$, Peres propõe um critério baseado na transposição parcial da matriz densidade $\hat{\rho}$ que pode ser assim enunciado:

\footnotetext{
${ }^{3}$ Aqui utilizamos a propriedade de transposição de produtos de matrizes, $\mathcal{T}(\mathcal{A B})=\mathcal{T}(\mathcal{B}) \mathcal{T}(\mathcal{A})$.
} 
Se a matriz $\hat{\rho}$ que descreve um sistema bipartite é separável, então o operador $\hat{\sigma}$ obtido pela transposição de um dos seus subsistemas (transposição parcial) é um operador positivo semi-definido (operador que possui todos os autovalores não negativos, $\hat{\sigma} \geq 0$ ).

Dito de outra forma:

Se o estado $\hat{\rho}$ é separável (equação 3.5.5), então $\hat{\rho}^{\mathcal{T}_{1}} \geq 0$ e $\hat{\rho}^{\mathcal{T}_{2}}=\left(\hat{\rho}^{\mathcal{T}_{1}}\right)^{\mathcal{T}} \geq 0$.

Na afirmação acima, a notação $\mathcal{T}_{j}$, com $j=1,2$, indica qual sistema está sendo transposto.

A fim de verificar essa assertiva, vamos considerar um sistema bipartite separável, cujo operador densidade que o descreve tem sua forma geral dada pela equação (3.5.5). Se fizermos uma transposição parcial no sistema 1, obtemos o operador

$$
\hat{\sigma}=\sum_{i} p_{i}\left[\hat{\rho}_{i}^{(1)}\right]^{\mathcal{T}} \otimes \hat{\rho}_{i}^{(2)}
$$

Dada a discussão anterior sobre mapas positivos, sabemos que $\left(\hat{\rho}_{i}^{(1)}\right)^{\mathcal{T}} \geq 0$, pois $\hat{\rho}_{i}^{(1)}$ é hermitiano positivo semi-definido. Dessa forma, mesmo que a operação de transposição parcial seja desprovida de significado físico, o operador $\hat{\sigma}$ pode ainda ter atributos de uma matriz densidade. Portanto, o operador $\hat{\sigma}$ deve fornecer autovalores não negativos, sendo esta a condição necessária que deve ser satisfeita para escrever $\hat{\rho}$ como uma soma de produtos diretos das matrizes $\hat{\rho}^{(1)}$ e $\hat{\rho}^{(2)}$.

Como exemplo, vamos considerar o operador densidade associado ao estado de Bell $|\Psi\rangle=1 / \sqrt{2}(|10\rangle+|01\rangle)$, expandido na base $\{|m, n\rangle\}$ ordenada como $\{00,01,10,11\}$,

$$
\hat{\rho}=\frac{1}{2}\left(\begin{array}{cccc}
0 & 0 & 0 & 0 \\
0 & 1 & 1 & 0 \\
0 & 1 & 1 & 0 \\
0 & 0 & 0 & 0
\end{array}\right) \stackrel{\mathcal{T}_{A}}{\longrightarrow} \frac{1}{2}\left(\begin{array}{cccc}
0 & 0 & 0 & 1 \\
0 & 1 & 0 & 0 \\
0 & 0 & 1 & 0 \\
1 & 0 & 0 & 0
\end{array}\right)
$$

Os autovalores de $\hat{\rho}$ são $\{0,0,0,1\}$, por outro lado os autovalores do operador obtido pela transposição parcial são $\{0.5,0.5,0.5,-0.5\}$, o que viola a positividade. Isto é suficiente para mostrar a inseparabilidade do estado de Bell.

No que diz respeito a circunstâncias específicas no âmbito de variáveis discretas, foi demonstrado pelos Horodecki [Horodecki M. 1996] que o critério PPT é necessário e suficiente, para sistemas compostos de baixa dimensão, $(2 \times 2)$ e $(2 \times 3)$. A notação $(N \times M)$ significa que o sistema é descrito pelo espaço de Hilbert $C^{N} \otimes C^{M}$, de modo que $(2 \times 2)$ corresponde, por exemplo, a sistema composto de duas partículas de spin $1 / 2$ (dois qubits) e $(2 \times 3)$ a um sistema composto por um subsistema de dois e outro de três níveis (um qubit e um qutrit).

Dada a baixa dimensionalidade demonstrada, podemos imaginar que o critério se torna impraticável em sistemas com dimensões elevadas, dada a quantidade de operações parciais necessárias. Contudo, no caso de sistemas descritos por variáveis contínuas a transposição parcial é facilmente aplicada, conforme será apresentado na próxima seção. 


\subsubsection{Variáveis contínuas}

A extensão do critério PPT para variáveis contínuas foi feita por Simon [Simon 2000], que deu uma interpretação geométrica para a transposição parcial em termos de uma reversão temporal (ou uma reflexão especular) no espaço de fase, permitindo obter relações de incerteza que devem ser obedecidas por todos os estados separáveis.

O desenvolvimento do critério é realizado para um sistema bipartite cujos constituintes são descritos pelos operadores $\hat{a}_{j}=\left(\hat{q}_{j}+i \hat{p}_{j}\right) / \sqrt{2}(j=1,2)$, onde $q_{j}$ e $p_{j}$ podem representar, por exemplo, operadores posição e momento de um estado EPR ${ }^{4}$. Estes operadores obedecem às regras usuais de comutação, $\left[\hat{a}_{i}, \hat{a}_{j}^{\dagger}\right]=\delta_{i j}$ e $\left[\hat{q}_{i}, \hat{p}_{j}\right]=2 i \delta_{i j}$.

Como estamos interessados em trabalhar no espaço de fase, é mais conveniente definir os vetores

$$
\hat{\xi}=\left(\hat{q}_{1}, \hat{p}_{1}, \hat{q}_{2}, \hat{p}_{2}\right)^{T}, \quad \text { e } \quad \xi=\left(q_{1}, p_{1}, q_{2}, p_{2}\right)^{T}
$$

onde à esquerda temos operadores e à direita variáveis canônicas. Com essa definição, as relações de comutação podem ser escritas em uma forma mais compacta conhecida, por razões que ficarão mais claras posteriormente, como forma simplética:

$$
\begin{aligned}
& {\left[\hat{\xi}_{\alpha}, \hat{\xi}_{\beta}\right]=2 i \Omega_{\alpha \beta} \quad, \quad \alpha, \beta=1,2,3,4 ;} \\
& \Omega=\left(\begin{array}{ll}
J & 0 \\
0 & J
\end{array}\right) \quad, \quad J=\left(\begin{array}{cc}
0 & 1 \\
-1 & 0
\end{array}\right) \text {. }
\end{aligned}
$$

Como mencionado no capítulo anterior, no espaço de fase, o estado do sistema é dado pela distribuição de Wigner que, para um sistema bipartite, se relaciona com a matriz densidade da seguinte forma:

$$
\begin{aligned}
W\left(q_{1}, q_{2}, p_{1}, p_{2}\right)=\frac{1}{\pi^{2}} & \int d p_{1}^{\prime} d p_{2}^{\prime}\left\langle p_{1}-p_{1}^{\prime}, p_{2}-p_{2}^{\prime}|\hat{\rho}| p_{1}+p_{1}^{\prime}, p_{2}+p_{2}\right\rangle \\
& \times \exp \left[2 i\left(p_{1}^{\prime} q_{1}+p_{2}^{\prime} q_{2}\right)\right] .
\end{aligned}
$$

A fim de ver o efeito da transposição parcial sobre a função anterior, consideremos a matriz densidade separável dada na equação (3.5.5). A função de Wigner (3.7.6) para este sistema é dada por

$$
\begin{aligned}
& W\left(q_{1}, q_{2}, p_{1}, p_{2}\right)=\frac{1}{\pi^{2}} \sum_{j} \tilde{p}_{j} \int d p_{1}^{\prime} d p_{2}^{\prime}\left\langle p_{1}-p_{1}^{\prime}, p_{2}-p_{2}^{\prime}\left|\hat{\rho}_{j}^{(1)} \otimes \hat{\rho}_{j}^{(2)}\right| p_{1}+p_{1}^{\prime}, p_{2}+p_{2}^{\prime}\right\rangle \\
& \times \exp \left[2 i\left(p_{1}^{\prime} q_{1}+p_{2}^{\prime} q_{2}\right)\right], \\
& W\left(q_{1}, q_{2}, p_{1}, p_{2}\right)=\frac{1}{\pi^{2}} \sum_{j} \tilde{p}_{j} \int d p_{1}^{\prime} d p_{2}^{\prime} \exp \left[2 i\left(p_{1}^{\prime} q_{1}+p_{2}^{\prime} q_{2}\right)\right] \\
& \times\left\langle p_{1}-p_{1}^{\prime}\left|\hat{\rho}_{j}^{(1)}\right| p_{1}+p_{1}^{\prime}\right\rangle\left\langle p_{2}-p_{2}^{\prime}\left|\hat{\rho}_{j}^{(2)}\right| p_{2}+p_{2}^{\prime}\right\rangle .
\end{aligned}
$$

\footnotetext{
${ }^{4}$ As grandezas de interesse neste trabalho também são rotuladas por $\hat{p}$ e $\hat{q}$, representando as quadraturas amplitude e fase do campo eletromagnético respectivamente.
} 
Ao realizar a transposição sobre o subsistema 2,

$$
\left\langle p_{2}-p_{2}^{\prime}\left|\left[\hat{\rho}_{j}^{(2)}\right]^{\mathcal{T}}\right| p_{2}+p_{2}^{\prime}\right\rangle=\left\langle p_{2}+p_{2}^{\prime}\left|\hat{\rho}_{j}^{(2)}\right| p_{2}-p_{2}^{\prime}\right\rangle,
$$

obtemos a função de Wigner

$$
\begin{aligned}
W^{\mathcal{T}_{2}}\left(q_{1}, q_{2}, p_{1}, p_{2}\right)=\frac{1}{\pi^{2}} \sum_{j} \tilde{p}_{j} & \int d p_{1}^{\prime} d p_{2}^{\prime} \exp \left[2 i\left(p_{1}^{\prime} q_{1}+p_{2}^{\prime} q_{2}\right)\right] \\
& \times\left\langle p_{1}-p_{1}^{\prime}\left|\hat{\rho}_{j}^{(1)}\right| p_{1}+p_{1}^{\prime}\right\rangle\left\langle p_{2}+p_{2}^{\prime}\left|\hat{\rho}_{j}^{(2)}\right| p_{2}-p_{2}^{\prime}\right\rangle .
\end{aligned}
$$

Após realizar a mudança de variável $p_{2}^{\prime} \rightarrow\left(-p_{2}^{\prime}\right)$ na equação precedente, fica simples perceber que as equações (3.7.10) e (3.7.8) se relacionam de modo bem definido,

$$
W^{\mathcal{T}_{2}}\left(p_{1}, q_{1}, p_{2}, q_{2}\right)=W\left(p_{1}, q_{1}, p_{2},-q_{2}\right)
$$

A partir dessa equação Simon interpretou geometricamente o efeito da transposição parcial em variáveis contínuas como uma reflexão especular de umas das variáveis canônicas do subsistema transposto. Alternativamente, podemos interpretar o resultado como uma reversão temporal no espaço de fases, ou seja, em termos da evolução temporal dada pela equação de Schrödinger, existe uma troca de sinal nas coordenadas de momento $(i \hbar \partial / \partial t \rightarrow-i \hbar \partial / \partial t)$ [Braunstein 2005].

Portanto, se $\hat{\rho}$ é separável, ao realizar uma transposição parcial relativa a um de seus subsistemas obtemos um novo operador densidade $\hat{\sigma}$ cuja função de Wigner é obtida da função de Wigner de $\hat{\rho}$ por uma reflexão especular da variável $q_{2}$. Isso corresponde a

$$
W\left(p_{1}, q_{1}, p_{2},-q_{2}\right)=W(\Upsilon \xi), \quad \Upsilon=\operatorname{diag}(1,1,1,-1) .
$$

Este resultado viabiliza a elaboração de um critério de separabilidade operacional, em termos da matriz de covariâncias do sistema. Para obtê-lo, é necessário expressar o vetor $\hat{\xi}$ dado na equação (3.7.4) como a soma do seu valor médio $(\xi)$ com um termo de flutuação $(\delta \hat{\xi}), \hat{\xi}=\xi+\delta \hat{\xi}$. As correlações entre suas componentes são organizadas numa matriz de covariâncias,

$$
\begin{aligned}
V_{\alpha \beta}=\frac{1}{2}\left\langle\left\{\delta \hat{\xi}_{\alpha}, \delta \hat{\xi}_{\beta}\right\}\right\rangle & =\frac{1}{2} \operatorname{Tr}\left\{\left\{\delta \hat{\xi}_{\alpha}, \delta \hat{\xi}_{\beta}\right\} \hat{\rho}\right\} \\
& =\int d^{4} \xi \delta \xi_{\alpha} \delta \xi_{\beta} W(\xi),
\end{aligned}
$$

que, para representar um estado físico, deve ser real $\left(V=V^{*}\right)$, simétrica $\left(V=V^{\mathcal{T}}\right)$ e positiva $(V \geq 0)$. Além disso, a matriz de covariâncias de um estado $\hat{\rho}$ qualquer, deve satisfazer a desigualdade:

$$
V+i \Omega \geq 0
$$

Conforme já explicado na referência [Rigolin 2005], a relação anterior pode ser demonstrada aplicando o teorema de Williamson [Williamson 1936] para um sistema bipartite: 
"Qualquer matriz de covariâncias $V_{4 \times 4}$ real, positiva e simétrica pode ser colocada em uma forma diagonal $V^{\prime}=\operatorname{diag}\left[\nu_{1}, \nu_{1}, \nu_{2}, \nu_{2}\right]$ por meio de uma transformação simplética $S \in S_{p}(4, R)$ dada por $S V S^{\mathcal{T}}=V^{\prime \prime}$. Dito de outra forma, existe uma transformação simplética que desacopla os subsistemas 1 e 2. Matematicamente,

$$
V^{\prime}=\left(\begin{array}{cccc}
\nu_{1} & 0 & 0 & 0 \\
0 & \nu_{1} & 0 & 0 \\
0 & 0 & \nu_{2} & 0 \\
0 & 0 & 0 & \nu_{2}
\end{array}\right)=\left(\begin{array}{cccc}
\Delta^{2} q_{1}^{\prime} & 0 & 0 & 0 \\
0 & \Delta^{2} p_{1}^{\prime} & 0 & 0 \\
0 & 0 & \Delta^{2} q_{2}^{\prime} & 0 \\
0 & 0 & 0 & \Delta^{2} p_{2}^{\prime}
\end{array}\right)
$$

Uma transformação simplética não altera as relações de comutação entre as variáveis canônicas, daí chamarmos a equação (3.7.5) de forma simplética. Dito isto, as relações de incerteza de Heisenberg continuam válidas:

$$
\Delta^{2} q_{i}^{\prime} \Delta^{2} p_{i}^{\prime} \geq 1 \Longrightarrow \nu_{i} \nu_{i} \geq 1 \Longrightarrow \nu_{i} \geq 1 .
$$

Portanto, dado que $\Omega^{\prime}=S \Omega S^{\mathcal{T}}=\Omega$, temos

$$
V^{\prime}+i \Omega^{\prime}=\left(\begin{array}{cccc}
\nu_{1} & i & 0 & 0 \\
-i & \nu_{1} & 0 & 0 \\
0 & 0 & \nu_{2} & i \\
0 & 0 & -i & \nu_{2}
\end{array}\right)
$$

Os autovalores obtidos após a diagonalização dessa matriz são

$$
\begin{array}{ccc}
\lambda_{1}=\nu_{1}+1 & , & \lambda_{2}=\nu_{1}-1 \\
\lambda_{3}=\nu_{2}-1 & \text { e } & \lambda_{4}=\nu_{2}+1 .
\end{array}
$$

Novamente, para que $V^{\prime}+i \Omega^{\prime} \geq 0$, os autovalores $\lambda_{i}$ devem ser positivos, o que implica $\nu_{i} \geq 1$. Por fim, sendo $V^{\prime}+i \Omega^{\prime}=S(V+i \Omega) S^{\mathcal{T}} \geq 0$, obtemos o resultado desejado $V+i \Omega \geq 0$. Assim, para que a matriz obtida pela transposição parcial possa representar um sistema físico, os autovalores simpléticos devem ser maiores que um. Este resultado é de grande importância para a formulação do critério de separabilidade.

Partindo da relação entre a matriz de covariâncias e a função de Wigner dada pela equação (3.7.13), é possível mostrar que a transposição parcial na matriz de covariâncias pode ser implementada através da transformação [Rigolin 2005]

$$
V \stackrel{\mathcal{T}_{2}}{\longrightarrow} \tilde{V}=\Upsilon V \Upsilon
$$

Mostramos anteriormente que ao realizarmos a transposição parcial em um sistema descrito pelo operador densidade $\hat{\rho}$ ou, de modo alternativo, pela função de Wigner $W(\xi)$, obtemos um novo operador $\hat{\sigma}$ que dá origem à função $W(\Upsilon \xi)$, também em condições de representar um sistema físico. Assim, após a transposição, a desigualdade

$$
\tilde{V}+i \Omega \geq 0
$$


deve ainda ser satisfeita, caso contrário representará um sistema não-físico. Essa é a essência do critério de separabilidade de Simon[Simon 2000].

Portanto, o cálculo dos autovalores simpléticos apresentado anteriormente deve ser suficiente para verificar se o sistema transposto dá origem a uma matriz de covariâncias legítima. Porém, na referência [Adesso 2006], pode ser encontrada uma forma ainda mais prática de aplicar critério de separabilidade descrito. Conforme demonstrado por Adesso, os autovalores simpléticos $\nu_{k}$ da matriz $V$ são dados por $\nu_{k}=\sqrt{o_{k}}$, onde $o_{k}$ são os autovalores da matriz $\mathcal{V}=-\left(V \Omega^{2}\right)$.

Temos agora um critério de fácil implementação que permite identificar se uma dada matriz de covariância $V$ descreve um estado emaranhado ou não. O critério PPT é para casos gerais um critério suficiente na determinação do emaranhamento e se torna também necessário para estados gaussianos bipartites do tipo $(1 \times 1)$, como demonstrado por Simon [Simon 2000], o mesmo podendo ser afirmado para sistemas analisados em bipartições do tipo $(1 \times N)$, verificando se um subsistema é separável dos outros $N$ [Werner 2001].

Como exemplo, considere uma matriz de covariâncias para um sistema bipartite na base $\left\{\hat{p}_{1}, \hat{q}_{1}, \hat{p}_{2}, \hat{q}_{2}\right\}$, também encontrado na referência [Cassemiro 2008],

$$
V=\left(\begin{array}{cccc}
S_{\hat{p}_{1}} & 0 & C_{\hat{p}_{1} \hat{p}_{2}} & 0 \\
0 & S_{\hat{q}_{1}} & 0 & C_{\hat{q}_{1} \hat{q}_{2}} \\
C_{\hat{p}_{2} \hat{p}_{1}} & 0 & S_{\hat{p}_{2}} & 0 \\
0 & C_{\hat{q}_{2} \hat{q}_{1}} & 0 & S_{\hat{q}_{2}}
\end{array}\right)=\left(\begin{array}{cccc}
2 & 0 & 1,8 & 0 \\
0 & 3 & 0 & -2,5 \\
1,8 & 0 & 2 & 0 \\
0 & -2,5 & 0 & 3
\end{array}\right)
$$

Realizar a transposição parcial no subsistema 2 é o mesmo que fazer a substituição $\delta \hat{q}_{2} \rightarrow$ $-\delta \hat{q}_{2}$. Isso resulta em

$$
\tilde{V}=\left(\begin{array}{cccc}
2 & 0 & 1,8 & 0 \\
0 & 3 & 0 & 2,5 \\
1,8 & 0 & 2 & 0 \\
0 & 2,5 & 0 & 3
\end{array}\right)
$$

Com essa matriz calculamos os autovalores simpléticos,

$$
-(\Omega \tilde{V})^{2}=\left(\begin{array}{cccc}
10,5 & 0 & 10,4 & 0 \\
0 & 10,5 & 0 & 10,4 \\
10,4,8 & 0 & 10,5 & 0 \\
0 & 10,4,5 & 0 & 10,5
\end{array}\right) \Rightarrow\left\{\tilde{\nu}_{k}\right\} \approx\{0,32 ; 0,32 ; 4,57 ; 4,57\}
$$

que evidencia o emaranhamento existente no sistema, dado $\nu_{1(2)}=0,32<1$. O estado proposto também viola o critério da soma de variâncias proposto por Duan [Duan 2000].

Temos portanto dois critérios bastante úteis para identificar o emaranhamento produzido em nosso sistema, o Oscilador Paramétrico Ótico (OPO). Apesar das demonstrações 
e exemplos dados neste capítulo ficarem restritos a situações mais simples, evolvendo estados bipartites, a extensão dos critérios para sistemas maiores não é complicada. $\mathrm{O}$ objetivo almejado foi dar uma ideia clara sobre o emaranhamento e apresentar os critérios de separabilidade utilizados nos estados produzidos em nosso experimento. Para mais detalhes, recomendamos consultar as referências [Villar 2007b, Cassemiro 2008].

No artigo EPR, o emaranhamento surgiu como uma propriedade que apontava o caráter incompleto da mecânica quântica. Após inúmeras discussões filosóficas, teorias que possibilitavam testes experimentais foram elaboradas e, uma vez comprovado, o emaranhamento passou a ser visto como recurso para o processamento de informação. Definimos formalmente essa característica intrínseca à mecânica quântica e apresentamos os critérios de soma de variâncias e positividade sob transposição parcial, bastante utilizados nesta tese. 


\section{Capítulo 4}

\section{O Oscilador Paramétrico Ótico}

How do you like to go up in a swing,

Up in the air so blue?

Oh, I do think it the pleasantest thing

Ever a child can do!

"The Swing" - Robert Louis Stevenson (1913)

Os primeiros efeitos não-lineares observados pela interação da luz com a matéria foram obtidos após a produção de luz monocromática intensa em masers de rubi no início da década de 1960 [Maiman 1960]. Na época, a produção de harmônicos óticos era apresentada como a possibilidade mais atraente, sendo realizada em 1961 por Franken et al. [Franken 1961]. Estes primeiros resultados deram impulso a uma intensa investigação teórica e desenvolvimentos de técnicas experimentais que visavam aumentar a eficiência de conversão da frequência da luz, principalmente relacionada com diferentes tipos de acordo de fase e focagem do laser [Armstrong 1962, Franken 1963].

Em 1962, a teoria desenvolvida por Kroll mostrou ser possível a criação de uma fonte de luz sintonizável com os lasers pulsados disponíveis na época, utilizando espelhos para aumentar a interação da luz com o meio não-linear [Kroll 1962]. A realização experimental aconteceu dois anos mais tarde graças a Giordmaine e Miller [Giordmaine 1965], seguida de demonstrações com lasers operando no regime contínuo (CW) utilizando uma cavidade ressonante para os dois campos conhecidos atualmente como sinal e complementar, gerados pela interação entre o feixe de bombeio e o meio não-linear [Smith 1968, Byer 1968]. Contudo, apesar de todos os esforços, o desenvolvimento estagnou até o fim da década de 1980, por causa da baixa qualidade espectral e espacial das fontes laser e dos cristais.

A situação mudou drasticamente a partir dos anos 90 com o surgimento de novas técnicas de fabricação de cristais não-lineares e com o aparecimento dos lasers de estado sólido, possibilitando a confecção de OPO de ressonância simples $(\mathrm{SRO})^{1}$ com altas efi-

\footnotetext{
${ }^{1}$ Singly Resonant Optical Parametric Oscillator.
} 
ciência de conversão, estabilidade e capacidade de sintonia [Bosenberg 1996]. Este foi o passo necessário para que este sistema despertasse interesse no mundo comercial.

Atualmente o Oscilador Paramétrico Ótico (OPO) desempenha um papel fundamental em técnicas de espectroscopia, como por exemplo espectroscopia molecular [Asvany 2007] e espectroscopia fotoquímica [Marushkevich 2011], dada sua característica sintonizável que permite trabalhar dentro de uma faixa de comprimento de onda que se estende de 1.3 a $4.5 \mu \mathrm{m}$. Além disso, pode ser aplicado em estudos de gases vestigiais, pois basta sintonizá-lo em uma região onde o gás ou múltiplos gases possuem forte absorção com baixa interferência com outros de diferentes tipos [Cristescu 2008, Herpen 2006]. São outras aplicações a investigação de metamateriais e a geração de feixes de luz com propriedades não clássicas [Villar 2005], sendo esta última o foco desta tese.

Feixes de luz com propriedades não clássicas merecem atenção devido à possibilidade de desenvolver protocolos que envolvem armazenamento e troca de informação, tais como codificação densa (dense coding) [Li 2002], teletransporte [Furusawa 1998], sistemas de criptografia [Silberhorn 2002] e comunicação entre sistemas físicos distintos como em uma rede quântica. À parte todo interesse tecnológico que tem sido o grande combustível da ciência nos dias atuais, igualmente importante é o desenvolvimento da física básica que neste contexto inclui a realização experimental de estados de Fock de um e dois fótons [Zavatta 2008] e produção de "pequenos gatos de Schrödinger" obtidos pela superposição de estados coerentes, assim denominados por conta da baixa amplitude dos estados em superposição [Ourjoumtsev 2007]. Outras técnicas que chamam atenção é a adição e subtração de fótons que permitem realizar testes experimentais dos alicerces da Mecânica Quântica [Parigi 2007], além de possibilitar a geração de estados de Gato de Schrödinger com grande amplitude [Marek 2008], sendo o OPO uma provável ferramenta neste desenvolvimento.

Neste capítulo, apresentaremos a descrição do OPO triplamente ressonante acima do limiar de oscilação. O OPO do Laboratório de Manipulação Coerente de Átomos e Luz (LMCAL) está em operação há mais de dez anos e vem sofrendo modificações à medida que novos fenômenos são descobertos. Dentre os resultados mais relevantes estão a produção de emaranhamento entre os feixes sinal e complementar com comprimentos de onda distintos [Villar 2005], demonstração teórica de emaranhamento tripartite [Villar 2006], medidas de correlações entre os feixes gêmeos e feixe de bombeio refletido [Cassemiro 2007], caracterização do ruído espúrio inserido por fônons da rede cristalina [César 2008b, Coelho 2009a], comprovação experimental de emaranhamento entre os feixes bombeio refletido, sinal e complementar [Coelho 2009b] e caracterização da dinâmica do emaranhamento frente a perdas [Barbosa 2010a].

Iniciaremos com uma descrição qualitativa seguida da descrição formal já apresentada em outras teses do grupo [Cassemiro 2008, Villar 2007b, Martinelli 2002]. Feito isto, apresentaremos a aplicação do critério de positividade sob transposição parcial e mostraremos 
as relações presentes entre momentos de ordem superior que devem ser satisfeitas por uma estatística gaussiana.

\subsection{Descrição qualitativa do OPO}

Um OPO é formado essencialmente de três elementos básicos: fonte de luz intensa (laser de bombeio), material não-linear com suscetibilidade de segunda ordem $\chi^{(2)}$ que atua como meio de ganho e uma cavidade ótica responsável pela realimentação ativa do meio. Uma descrição qualitativa do princípio de operação de um OPO é dada com o auxílio da figura 4.1 .

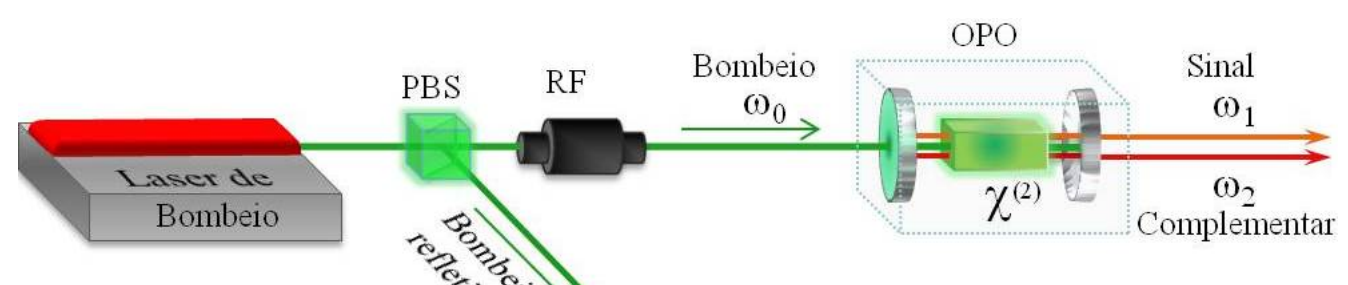

Figura 4.1: Descrição esquemática de um Oscilador Paramétrico Ótico. Uma fonte de luz laser fornece o campo de bombeio de frequência $\omega_{0}$ que interage com um cristal de suscetibilidade não-linear $\chi^{(2)}$ disposto dentro de uma cavidade Fabry-Perot, onde há a criação de dois novos campos de frequência $\omega_{1}$ e $\omega_{2}$. O processo de interação também dá origem a um campo refletido de frequência igual a do feixe de bombeio, sendo por essa razão denominado bombeio refletido, separado do campo de bombeio incidente por polarização, utilizando um Rotator de Faraday (RF) e um PBS.

Os fótons do feixe de entrada (bombeio) de frequência $\omega_{0}$, ao interagirem com o cristal não-linear, são convertidos a uma certa taxa em dois outros fótons que constituem os feixes sinal e complementar de frequências mais baixas $\omega_{1}$ e $\omega_{2}$, respectivamente. O efeito se dá pelo processo de conversão paramétrica descendente (CPD) que ocorre por conta da relação existente entre a polarização e o campo elétrico no meio. O campo elétrico incidente $\vec{E}_{b}(\mathbf{r}, t)$ no cristal não linear dá origem a uma polarização $\mathcal{P}(\mathbf{r}, t)$ dependente de termos de ordem mais alta do campo,

$$
\mathcal{P}(\mathbf{r}, t)=\varepsilon_{0} \chi^{(1)} \vec{E}(\mathbf{r}, t)+\varepsilon_{0} \chi^{(2)} \vec{E}^{2}(\mathbf{r}, t)+\varepsilon_{0} \chi^{(3)} \vec{E}^{3}(\mathbf{r}, t)+\ldots,
$$

sendo de interesse para nossa discussão o termo de segunda ordem $\chi^{(2)}$, nulo em materiais centrossimétricos, assim classificados por possuírem simetria de inversão com relação à origem das coordenadas dos átomos que compõem o meio $(\overrightarrow{\mathbf{r}} \rightarrow-\overrightarrow{\mathbf{r}}$ não altera a resposta à polarização no material).

O processo de aniquilação e criação de fótons deve satisfazer a conservação de energia, ou seja, a soma das frequências dos feixes produzidos deve ser igual à frequência do feixe 
de bombeio,

$$
\omega_{0}=\omega_{1}+\omega_{2}
$$

onde os índices 0, 1 e 2 denotam respectivamente os feixes bombeio, sinal e complementar, notação que será mantida até o fim da tese. Além disso, para que seja obtida a máxima taxa de conversão de fótons possível, mostra-se a necessidade de um vínculo entre os vetores de onda dos campos gerados,

$$
\overrightarrow{\mathbf{k}}_{0}=\overrightarrow{\mathbf{k}}_{1}+\overrightarrow{\mathbf{k}}_{2}
$$

As condições (4.1.2) e (4.1.3) são conhecidas como acordo de frequência (frequencymatching) e fase (phase-matching), representadas na figura 4.2. Esta última é obtida em nosso experimento escolhendo um ângulo apropriado entre a direção de propagação dos campos e os eixos óticos do cristal birrefringente, além do controle fino de temperatura do meio. O acordo de fase que obtemos é do tipo II: a polarização dos campos gerados são ortogonais entre si.

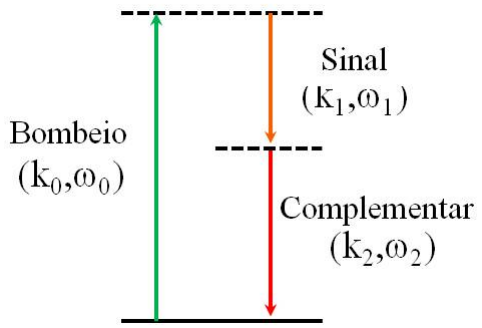

Figura 4.2: Representação do processo paramétrico em um sistema de três níveis. O fóton do campo de bombeio de frequência $\omega_{0}$ é aniquilado dando origem a dois outros fótons de frequências mais baixas $\omega_{1}$ e $\omega_{2}$. Os níveis pontilhados indicam o carácter sintonizável do OPO, obtido pelas condições de acordo de fase, seja pela alteração do nível correspondente ao bombeio ou do nível intermediário referente aos feixes gêmeos.

A taxa de conversão se torna consideravelmente maior quando inserimos o cristal dentro de uma cavidade ótica, responsável pela realimentação do mesmo. O cristal passa a atuar como meio de ganho para os feixes sinal e complementar, amplificados pela transferência de energia do feixe de bombeio. A oscilação tem início quando a intensidade do bombeio ultrapassa uma potência característica denominada potência de limiar $\left(P_{\text {lim }}\right)$, o que significa dizer que o ganho associado ao processo de conversão superou as perdas totais geradas pela transmissão dos espelhos, absorção, espalhamento e difração. Ao atingir o limiar de oscilação, parte da energia do bombeio é transferida para os feixes sinal e complementar, diminuindo a intensidade do mesmo dentro do cristal acarretando uma saturação do ganho. Este processo faz o OPO funcionar em um regime estacionário.

Em especial, isso pode ser visto no tratamento clássico feito por Debuisschert et al. [Debuisschert 1993] para o OPO tipo-II (sinal e complementar com polarizações ortogonais). Além deste trabalho, descrições detalhadas sobre o funcionamento do OPO 
podem ser obtidas em [Breunig 2011, Träger 2007], sendo sua física descrita de modo mais fundamental em alguns livros de ótica não-linear [Grynberg 2010, Yariv 1989].

O desempenho dos OPOs no que diz respeito à sua capacidade de sintonia, potência de saída dos campos e coerência depende diretamente de sua estrutura básica composta pelo laser de bombeio, cristal não-linear e cavidade ótica. O cristal deve ter dimensões e coeficiente não-linear otimizados para uma faixa de frequência específica. Sendo assim, o regime de operação desejado é obtido escolhendo adequadamente o feixe de bombeio e os parâmetros da cavidade. O OPO pode ser ressonante para um (SRO), dois (DRO) ou para os três (TRO) campos. A grande vantagem do primeiro é a possibilidade de sintonia contínua dos comprimentos de onda, porém com a necessidade de um bombeio da ordem de alguns watts, sendo utilizado no regime pulsado [Yang 1993]. Para o caso duplamente ressonante, a potência de limiar é duas ou três vezes menor, porém começam a surgir problemas de estabilidade que devem ser compensados por controles eletrônicos ativos [Falk 1971]. Nosso OPO opera no regime contínuo em uma configuração triplamente ressonante, ou seja, bombeio, sinal e complementar ressoam dentro da cavidade. As características principais desta configuração são a baixa potência de limiar (da ordem de alguns $\mathrm{mW}$ ) necessária para colocar o sistema em oscilação e a presença de correlações quânticas entre os feixes de saída.

Correlações entre os fótons oriundos de processos paramétricos foram pela primeira vez identificadas mostrando coincidências na detecção de fótons gerados por fluorescência paramétrica de um cristal não-linear [Burnham 1970]. O resultado obtido atesta que os fótons são realmente gerados aos pares pela aniquilação de um único fóton, fato que motivou experimentos visando medir correlações de intensidade entre campos por eles formados.

Inserindo o cristal não-linear em uma cavidade ótica, foi observada por Heidmann et al. [Heidmann 1987] a compressão de ruído na subtração das intensidades cerca de $30 \%$ abaixo do shot-noise, característica que levou à designação "feixes gêmeos" para sinal e complementar. Após sua verificação experimental em OPOs operando abaixo do limiar de oscilação, o nível de compressão de ruído com relação ao ruído quântico padrão tem aumentado progressivamente atingindo $-4 \mathrm{~dB}$ [Wu 1986], com recorde atual da ordem de $-9,5 \mathrm{~dB}$ [Laurat 2005]. Mais tarde, foi demonstrado que a conservação de energia envolvida no processo de criação dos pares de fótons permite mostrar que as fases dos campos apresentam forte anti-correlação [Reid 1988]. Essas correlações são os ingredientes chave para a existência de emaranhamento entre os campos produzidos pelo OPO.

Em nosso grupo foi realizada a primeira demonstração de emaranhamento bipartite entre os feixes sinal e complementar não degenerados em frequência acima do limiar de oscilação [Villar 2005]. Até a metade da década de 90, correlações envolvendo o feixe de bombeio refletido e os gêmeos tinham sido desprezadas, tendo despertado grande interesse com a previsão teórica de emaranhamento tripartite envolvendo os feixes bombeio 
refletido, sinal e complementar produzidos por um OPO triplamente ressonante acima do limiar de oscilação [Villar 2006].

Tendo em vista este fenômeno, foi investigada a existência de correlações quânticas entre os três campos, confirmadas experimentalmente por Cassemiro et al. [Cassemiro 2007]. Contudo, os resultados não foram suficientes para demonstrar experimentalmente o emaranhamento tripartite. O grande empecilho para atingir o tão almejado objetivo foi a presença de fônons na rede cristalina que atuam como uma fonte de ruído clássico para as quadraturas dos campos [César 2008b], mascarando o emaranhamento. Sendo assim, o controle e a redução do ruído espúrio passam a ter um papel crucial na verificação do fenômeno. As primeiras evidências de emaranhamento tripartite vieram em 2009, servindo como indicadoras paras as melhorias que deveriam ser implementadas no sistema [Coelho 2009a].

Apresentaremos nesta tese medidas definitivas de emaranhamento entre os três campos produzidos pelo OPO acima do limiar de oscilação. Portanto, à luz do tratamento já apresentado em teses recentes do grupo [Villar 2007b, Cassemiro 2008], vamos descrever aqui a fonte desses estados emaranhados.

\subsection{Descrição teórica do OPO}

Nosso objeto de estudo é o sistema composto pelos três campos produzidos por um OPO acima do limiar de oscilação. O ponto de partida para geração desses campos é a entrada do laser de bombeio na cavidade ótica, onde há a interação com o cristal não-linear responsável pelo processo de conversão paramétrica descendente: fótons do bombeio são aniquilados gerando aos pares os fótons dos feixes sinal e complementar. Portanto, a cavidade ótica deve ter uma transmissão que permita tanto a entrada do bombeio quanto a saída dos campos onde serão observados os efeitos da interação nãolinear. Desse modo, também ocorre a interação do sistema quântico de interesse com o "ambiente", denominado reservatório, que acopla modos externos aos modos dos campos intracavidade por meio das transmissões. Sendo assim, o tratamento quântico adequado para a evolução desse conjunto se dá por meio da descrição de sistemas quânticos abertos [Leonhardt 2010].

Além disso, em um sistema físico real estão presentes perdas inevitáveis sobre as quais não temos controle, tais como absorção de luz pelo cristal e espalhamento gerados por imperfeições na superfície dos espelhos, denominadas perdas espúrias. Dessa forma, o processo mais geral existente no OPO é fundamentalmente irreversível. Em vista disso, a dinâmica deste sistema é regida pela equação de movimento conhecida como equação 
mestra, escrita como

$$
\begin{aligned}
i \hbar \frac{d}{d t} \hat{\rho} & =\left[\left(\hat{H}_{I}+\hat{H}_{\mathrm{in}}+\hat{H}_{0}+\hat{H}_{1}+\hat{H}_{2}\right), \hat{\rho}\right]+\sum_{j} \hat{\Lambda}_{j} \hat{\rho}, \\
\hat{\Lambda}_{j} \hat{\rho} & =\frac{\gamma_{j}^{\prime}}{\tau}\left(2 \hat{a}_{j} \hat{\rho} \hat{a}_{j}^{\dagger}-\hat{a}_{j}^{\dagger} \hat{a}_{j} \hat{\rho}-\hat{\rho} \hat{a}_{j}^{\dagger} \hat{a}_{j}\right),
\end{aligned}
$$

que nos dá a evolução do operador densidade $\hat{\rho}(t)$ do sistema. Vejamos agora cada um dos termos que compõem a equação acima.

A hamiltoniana $H_{I}$ refere-se ao processo de mistura de três ondas, que descreve a criação de um par de fótons de frequência $\omega_{1}$ e $\omega_{2}$ a partir da aniquilação de um fóton de frequência $\omega_{0}$ ou o processo contrário; criação de $\omega_{0}$ pela aniquilação de $\omega_{1}$ e $\omega_{2}$. Como o cristal de suscetibilidade não-linear $\chi^{(2)}$ é colocado dentro de uma cavidade, esses dois caminhos podem ocorrer. Dito isto, a hamiltoniana de interação tem sua expressão dada por [Walls 1995]

$$
\hat{H}_{I}=2 i \hbar \frac{\chi}{\tau}\left(\hat{a}_{0}^{\dagger} \hat{a}_{1} \hat{a}_{2}-\hat{a}_{0} \hat{a}_{1}^{\dagger} \hat{a}_{2}^{\dagger}\right)
$$

onde $\tau$ está associado com o intervalo espectral livre (FSR), diretamente relacionado com tempo que um fóton leva para dar uma volta completa dentro da cavidade e $\chi$ carrega consigo o coeficiente $\chi^{(2)}$ e as condições de acordo de fase, que expressam a magnitude do acoplamento.

O segundo termo $\hat{H}_{\text {in }}$ representa a injeção de um campo de bombeio intenso monocromático, considerado clássico e de amplitude média $\alpha_{\text {in }}^{0}$ real, que interage com o campo intracavidade de mesma frequência. Sua forma é

$$
\hat{H}_{\text {in }}=i \hbar \frac{\sqrt{2 \gamma_{0}}}{\tau} \alpha_{0}^{\text {in }}\left(\hat{a}_{0}-\hat{a}_{0}^{\dagger}\right)
$$

em que $\gamma_{0}$ representa a transmissividade do espelho de acoplamento para o campo de bombeio incidente.

As três hamiltonianas seguintes se referem aos modos quantizados intracavidade, escritas na representação de interação como

$$
\hat{H}_{j}=\hbar \frac{2 \gamma_{j}^{\prime}}{\tau} \Delta_{j} \hat{a}_{j}^{\dagger} \hat{a}_{j}, \quad \text { com } \quad j=0,1 \text { e } 2 .
$$

A quantidade $\Delta_{j}=\left(\omega_{j}-\omega_{c j}\right) / \delta \omega_{c j}$ é a dessintonia entre a frequência da cavidade $\omega_{c j}$ mais próxima da frequência do modo oscilante $\omega_{j}$, normalizada pela largura de banda da cavidade $\delta \omega_{c j}$. Os coeficientes $\gamma_{j}$ representam as perdas geradas pelo espelho de acoplamento, iguais à metade das transmissões dos espelhos para cada modo, $T=2 \gamma_{j}$. Em nosso experimento, a diferença entre os comprimentos de onda dos feixes gêmeos é da ordem de $\Delta \lambda_{(1,2)}=5 \mathrm{~nm}$, de modo que podemos considerar, sem perder em generalidade, 
$\gamma_{1}=\gamma_{2}=\gamma$. Definimos as perdas totais como $\gamma_{j}^{\prime}=\gamma_{j}+\mu_{j}$, onde $\mu_{j}$ representa as perdas espúrias no sistema, dadas por imperfeições na superfície dos espelhos ou na própria estrutura do cristal, que ocasionam espalhamento ou absorção da luz ${ }^{2}$.

No último termo da equação (4.2.1) aparecem os operadores de Lindblad $\hat{\Lambda}_{j}$, decorrentes das perdas oriundas do acoplamento do sistema de interesse com um reservatório formado por infinitos osciladores harmônicos desacoplados [Gardiner 1991]. No domínio de frequências óticas podemos colocar o reservatório à temperatura nula, o que justifica sua composição por modos de vácuo [Walls 1995].

O passo seguinte é resolver a equação (4.2.1). Fazemos isso reescrevendo-a em termos de funções de quase-probabilidade [Leonhardt 2010]. Em nosso caso, por causa de sua interpretação semiclássica intuitiva, escolhemos a função de Wigner. A substituição dos operadores, em um ordenamento específico, por amplitudes complexas é feita através das regras de correspondência entre a função $W(\vec{\alpha})$, onde $\vec{\alpha}=\left(\alpha_{0}, \alpha_{0}^{*}, \alpha_{1}, \alpha_{1}^{*}, \alpha_{2}, \alpha_{2}^{*}\right)$, e produtos envolvendo os operadores $\hat{a}_{j}, \hat{a}_{j}^{\dagger}$ e $\hat{\rho}$,

$$
\begin{aligned}
& \hat{a}_{j} \hat{\rho} \longleftrightarrow\left(\alpha_{j}+\frac{1}{2} \frac{\partial}{\partial \alpha_{j}^{*}}\right) W, \quad \hat{a}_{j}^{\dagger} \hat{\rho} \longleftrightarrow\left(\alpha_{j}^{*}-\frac{1}{2} \frac{\partial}{\partial \alpha_{j}}\right) W, \\
& \hat{\rho} \hat{a}_{j} \longleftrightarrow\left(\alpha_{j}-\frac{1}{2} \frac{\partial}{\partial \alpha_{j}^{*}}\right) W, \quad \hat{\rho} \hat{a}_{j}^{\dagger} \longleftrightarrow\left(\alpha_{j}^{*}+\frac{1}{2} \frac{\partial}{\partial \alpha_{j}}\right) W,
\end{aligned}
$$

obtidas utilizando a descrição alternativa da função de Wigner escrita a partir da função característica $\chi(\beta)=\operatorname{Tr}\{D \hat{\rho}\}$, onde $D=\exp \left(\beta \hat{a}^{\dagger}-\beta^{*} \hat{a}\right)$ [Walls 1995, Scully 1997]. Desta maneira, obtemos para a equação (4.2.1)

$$
\begin{aligned}
\frac{d}{d t} W(\vec{\alpha}) & =\left[\sum_{j} \gamma_{j}\left(1+i \Delta_{j}\right)\left(\frac{\partial}{\partial \alpha_{j}^{*}} \alpha_{j}^{*}-\frac{\partial}{\partial \alpha_{j}} \alpha_{j}\right)\right. \\
& +2 \chi\left(\alpha_{1} \alpha_{2} \frac{\partial}{\partial \alpha_{0}^{*}}+\alpha_{1}^{*} \alpha_{2}^{*} \frac{\partial}{\partial \alpha_{0}}-\alpha_{0} \alpha_{1} \frac{\partial}{\partial \alpha_{2}}-\alpha_{0}^{*} \alpha_{1} \frac{\partial}{\partial \alpha_{2}^{*}}-\alpha_{0} \alpha_{2}^{*} \frac{\partial}{\partial \alpha_{1}}-\alpha_{0}^{*} \alpha_{2} \frac{\partial}{\partial \alpha_{1}^{*}}\right) \\
& \left.-\sqrt{2 \gamma_{0}} \alpha_{i n}\left(\frac{\partial}{\partial \alpha_{0}^{*}}+\frac{\partial}{\partial \alpha_{0}}\right)+\sum_{j} \gamma_{j}^{\prime} \frac{\partial^{2}}{\partial \alpha_{j} \alpha_{j}^{*}}-\frac{\chi}{2}\left(\frac{\partial^{3}}{\partial \alpha_{0}^{*} \alpha_{1}^{*} \alpha_{2}^{*}}+\frac{\partial^{3}}{\partial \alpha_{0} \alpha_{1} \alpha_{2}}\right)\right] W(\vec{\alpha}) .
\end{aligned}
$$

A equação diferencial acima se torna uma equação de Fokker-Planck da função de Wigner quando o termo de derivada terceira é desprezado. Apesar deste termo carecer de interpretação física, sua omissão não traz consequências evidentes. Isso foi demonstrado na referência [Villar 2007a], onde pode ser encontrada uma análise completa utilizando a representação P-positiva, mostrando que os resultados obtidos nas duas representações são completamente equivalentes. Seguindo o formalismo descrito nas referências [Walls 1995, Gardiner 1991, Scully 1997], a equação de Fokker-Planck para $W(\vec{\alpha})$ é equivalente a um

\footnotetext{
${ }^{2}$ Apesar de ter sua origem em processos sobre os quais não temos controle, as perdas espúrias podem ser quantificadas.
} 
conjunto de equações de Langevin para as variáveis $\alpha_{j}$. As equações obtidas são

$$
\begin{aligned}
\tau \frac{d}{d t} \alpha_{0} & =-\gamma_{0}^{\prime}\left(1-i \Delta_{0}\right) \alpha_{0}-2 \chi \alpha_{1} \alpha_{2}+\sqrt{2 \gamma_{0}} \alpha_{0}^{\text {in }}+\sqrt{2 \mu_{0}} \delta v_{0} \\
\tau \frac{d}{d t} \alpha_{1} & =-\gamma^{\prime}(1-i \Delta) \alpha_{1}+2 \chi \alpha_{0} \alpha_{2}^{*}+\sqrt{2 \gamma} \delta u_{1}+\sqrt{2 \mu} \delta v_{1}, \\
\tau \frac{d}{d t} \alpha_{2} & =-\gamma^{\prime}(1-i \Delta) \alpha_{2}+2 \chi \alpha_{0} \alpha_{1}^{*}+\sqrt{2 \gamma} \delta u_{2}+\sqrt{2 \mu} \delta v_{2},
\end{aligned}
$$

sendo as forças de Langevin representadas pelos termos $\delta v_{j}$ e $\delta u_{j}$, flutuações do vácuo acopladas ao sistema devido às perdas espúrias e à transmissão dos espelhos, respectivamente. Para o feixe de bombeio, o termo $\delta u_{0}$ é substituído pela amplitude complexa $\alpha_{0}^{i n}$, que traz consigo flutuações acrescidas pelo feixe de bombeio incidente, que podem ser maiores ou iguais ao ruído padrão. Como estamos interessados em campos intensos, cujas flutuações são muito menores que o valor médio do campo, realizaremos uma análise linearizada reescrevendo as amplitudes $\alpha_{j}$ como uma soma de uma parte estacionária, correspondente ao valor médio, mais um termo de flutuação,

$$
\alpha_{j}=\bar{\alpha}+\delta \alpha_{j}, \alpha=\left\langle\bar{\alpha}_{j}\right\rangle=p_{j} e^{i \varphi_{j}},
$$

onde as fases $\varphi_{j}$ são tomadas em relação à fase do feixe de bombeio incidente $\left(\varphi_{0}^{i n}\right)$, escolhida nula. Obtidas as equações de Langevin, podemos estudar o caso estacionário e as flutuações quânticas dos campos produzidos pelo OPO.

\subsection{Soluções Estacionárias}

Ao tomar a média temporal das equações (4.2.9)-(4.2.11) obtemos as seguintes equações para os estados estacionários

$$
\begin{aligned}
\gamma_{0}^{\prime}(1-i \Delta) p_{0} e^{i \varphi_{0}}+2 \chi p^{2} e^{i \varphi_{+}}-\sqrt{2 \gamma_{0}} p_{0}^{i n}=0 \\
\gamma^{\prime}(1-i \Delta)-2 \chi p_{0} e^{-i\left(\varphi_{0}-\varphi_{+}\right)}=0 .
\end{aligned}
$$

As expressões acima são obtidas definindo $\varphi_{+}=\varphi_{1}+\varphi_{2}$ e $p_{1}=p_{2}=p$, esta última válida para o caso em que os feixes sinal e complementar são submetidos a perdas iguais.

Acima do limiar de oscilação, a intensidade do feixe de bombeio intracavidade é constante, algo visto por meio da equação (4.3.2) de onde obtemos

$$
p_{0}^{2}=\frac{\gamma^{\prime 2}}{4 \chi^{2}}\left(1+\Delta^{2}\right)
$$

independente da potência do bombeio incidente. Podemos ainda obter da equação anterior com auxílio de (4.3.2) a relação entre a fase do bombeio e a soma das fases dos feixes sinal e complementar,

$$
e^{i \varphi_{0}}=\frac{1-i \Delta}{\sqrt{1+\Delta^{2}}} e^{i \varphi_{+}} .
$$


É importante notar que nenhuma equação para a subtração da fase relativa entre os feixes sinal e complementar $\left(\varphi_{-}\right)$foi obtida, uma vez que esta não é bem definida. Isso se deve à existência de difusão de fases nestes campos [Reid 1989] o que não possibilita uma solução estacionária para suas amplitudes. Apesar disso ser fruto da linearização adotada, foi demonstrado via simulação numérica que os resultados são idênticos aos obtidos na representação $P$ [Villar 2007a]. Além disso, a duração da medida é muito mais rápida que a escala de tempo da difusão de fases, fato que torna a linearização viável. A análise quantitativa das escalas de tempo envolvidas é feita em detalhe na referência [Villar 2007b].

Substituindo a equação (4.3.4) em (4.3.2) e utilizando (4.3.3), obtemos a expressão

$$
p^{2}+\frac{\gamma_{0}^{\prime} \gamma^{\prime}}{4 \chi^{2}}\left(1-i \Delta_{0}\right)(1-i \Delta)=\frac{\sqrt{2 \gamma_{0}}}{2 \chi} p_{0}^{i n} e^{-i \varphi_{+}},
$$

cujo módulo ao quadrado permite encontrar a intensidade intracavidade $p^{2}$ dos feixes sinal e complementar,

$$
p^{2}=\sqrt{\frac{2 \gamma_{0}}{4 \chi^{2}} p_{0}^{\text {in } 2}-\left[\frac{\gamma_{0}^{\prime} \gamma^{\prime}}{4 \chi^{2}}\left(\Delta_{0}+\Delta\right)\right]^{2}}-\frac{\gamma_{0}^{\prime} \gamma^{\prime}}{4 \chi^{2}}\left(1-\Delta_{0} \Delta\right) .
$$

Essa equação fornece dois parâmetros bastante importantes para a análise dos resultados experimentais, a potência de limiar $\left(P_{l i m}\right)$ e a eficiência de conversão máxima do OPO $\left(\eta_{O P O}\right)$.

Quando a potência de bombeio supera a potência de limiar os feixes intensos sinal e complementar são gerados. Portanto, podemos obter sua expressão impondo $p=0$ e $\Delta=\Delta_{0}=0$ na equação $(4.3 .6)$, o que resulta em

$$
P_{\text {lim }}=\frac{\gamma_{0}^{\prime 2} \gamma^{\prime 2}}{8 \gamma_{0} \chi^{2}}
$$

A grandeza $P_{\text {lim }}$ é facilmente mensurável, basta observar os sinais gerados pelos fotodetectores dos feixes gêmeos diminuindo gradativamente a potência de bombeio até o ponto em que sinal e complementar não sejam mais observados. Utilizando um detector térmico obtemos o valor da potência de limiar, onde está incluída a constante $\chi$ relacionada com a suscetibilidade não-linear do meio. Além disso, $P_{\text {lim }}$ é dada pelas perdas totais envolvidas no processo de conversão paramétrica, de modo que seu valor serve como uma referência para garantir que estamos mantendo as características fundamentais do OPO em diferentes condições de operação, como por exemplo fixando a temperatura do cristal em valores distintos.

Ao substituir a expressão para $P_{\text {lim }}$ nas equações (4.3.3) e (4.3.6), obtemos as expressões para os valores estacionários,

$$
\begin{aligned}
& p^{2}=\frac{2 \gamma_{0}}{\gamma_{0}^{\prime} \gamma^{\prime}} P_{l i m}\left[\sqrt{\sigma-\left(\Delta_{0}+\Delta\right)^{2}}-\left(1-\Delta_{0} \Delta\right)\right], \\
& p_{0}^{2}=\frac{2 \gamma_{0}}{\gamma_{0}^{\prime 2}} P_{l i m}\left(1+\Delta^{2}\right),
\end{aligned}
$$


onde definimos a potência de bombeio relativa ao limiar de oscilação $\sigma=\left(p_{0}^{i n}\right)^{2} / P_{\text {lim }}$. Todos os gráficos de ruído quântico e dos critérios de separabilidade serão apresentados em função dessa grandeza. Finalmente, a potência dos campos sinal e complementar medidos pelos fotodetectores é igual à intensidade intracavidade dos mesmos multiplicada pela transmissão do espelho de saída,

$$
P=2 \gamma p^{2}=4 \eta_{O P O} P_{l i m}\left[\sqrt{\sigma-\left(\Delta_{0}+\Delta\right)^{2}}-\left(1-\Delta_{0} \Delta\right)\right]
$$

em que

$$
\eta_{O P O}=\frac{\gamma_{0} \gamma}{\gamma_{0}^{\prime} \gamma^{\prime}}
$$

é a eficiência de conversão máxima do OPO. Este parâmetro é muito importante para fazer a conversão da soma da intensidade dos feixes sinal e complementar para $\sigma$. Seu valor é obtido medindo as potências dos feixes gêmeos para diferentes valores de potência de bombeio, organizando os dados em um gráfico onde é ajustada a função (4.3.10), cujo parâmetro livre é justo $\eta_{O P O}$.

Como estamos trabalhando com feixes intensos, os resultados obtidos para os valores médios dos campos concordam com os resultados clássicos obtidos por Debuisschert et al. [Debuisschert 1993]. As flutuações quânticas serão discutidas na seção seguinte.

\subsection{Flutuações Quânticas Linearizadas}

Para estudar as flutuações quânticas, devemos reescrever as equações (4.2.9)-(4.2.11) na forma linearizada, que consiste em substituir a amplitude dos campos pela soma de seus valores médios com termos de flutuação (equação 4.2.12). Como estamos lidando com campos intensos, as flutuações são muito menores que o valor médio $|\delta \alpha(t)| \ll|\alpha(t)|$ o que justifica desprezar termos de segunda ordem no desenvolvimento. Ao fazer isso, obtemos as equações

$$
\begin{gathered}
\tau \frac{d}{d t} \delta \alpha_{0}=-\gamma_{0}^{\prime}\left(1-i \Delta_{0}\right) \delta \alpha_{0}-2 \chi p\left(\mathrm{e}^{i \varphi_{2}} \delta \alpha_{1}+\mathrm{e}^{i \varphi_{1}} \delta \alpha_{2}\right)+\sqrt{2 \gamma_{0}} \delta \alpha_{0}^{\text {in }}+\sqrt{2 \mu_{0}} \delta v_{0} \\
\tau \frac{d}{d t} \delta \alpha_{1}=-\gamma^{\prime}(1-i \Delta) \delta \alpha_{1}+2 \chi\left(p \mathrm{e}^{-i \varphi_{2}} \delta \alpha_{0}+p_{0} \mathrm{e}^{i \varphi_{0}} \delta \alpha_{2}^{*}\right)+\sqrt{2 \gamma} \delta u_{1}+\sqrt{2 \mu} \delta v_{1} \\
\tau \frac{d}{d t} \delta \alpha_{2}=-\gamma^{\prime}(1-i \Delta) \delta \alpha_{2}+2 \chi\left(p \mathrm{e}^{-i \varphi_{1}} \delta \alpha_{0}+p_{0} \mathrm{e}^{i \varphi_{0}} \delta \alpha_{1}^{*}\right)+\sqrt{2 \gamma} \delta u_{2}+\sqrt{2 \mu} \delta v_{2}
\end{gathered}
$$

que podem ser escritas em termos das flutuações das quadraturas amplitude e fase dos campos através da relação

$$
\delta \alpha_{j}=\frac{e^{i \varphi_{j}}}{2}\left(\delta p_{j}+i \delta q_{j}\right)
$$


Substituindo (4.4.4) em (4.4.1)-(4.4.3) e separando as partes real e imaginária, obtemos as seguintes equações para as flutuações das quadraturas

$$
\begin{aligned}
& \tau \frac{d}{d t} \delta p_{0}=-\gamma_{0}^{\prime} \delta p_{0}-\Delta_{0} \gamma_{0}^{\prime} \delta q_{0}-\gamma^{\prime} \beta \delta p_{1}+\Delta \gamma^{\prime} \beta \delta q_{1}-\gamma^{\prime} \beta \delta p_{2}+\Delta \gamma^{\prime} \beta \delta q_{2}+ \\
& +\sqrt{2 \gamma_{0}} \delta p_{0}^{i n}+\sqrt{2 \mu_{0}} \delta v_{p_{0}} \\
& \tau \frac{d}{d t} \delta q_{0}=\Delta_{0} \gamma_{0}^{\prime} \delta p_{0}-\gamma_{0}^{\prime} \delta q_{0}+\Delta \gamma^{\prime} \beta \delta p_{1}-\gamma^{\prime} \beta \delta q_{1}+\Delta \gamma^{\prime} \beta \delta p_{2}-\gamma^{\prime} \beta \delta q_{2}+ \\
& +\sqrt{2 \gamma_{0}} \delta q_{0}^{i n}+\sqrt{2 \mu_{0}} \delta v_{q_{0}} \\
& \tau \frac{d}{d t} \delta p_{1} \quad=\gamma^{\prime} \beta \delta p_{0}+\Delta \gamma^{\prime} \beta \delta q_{0}-\gamma^{\prime} \delta p_{1}-\Delta \gamma^{\prime} \delta q_{1}+\gamma^{\prime} \delta p_{2}-\Delta \gamma^{\prime} \delta q_{2}+ \\
& +\sqrt{2 \gamma} \delta u_{p_{1}}+\sqrt{2 \mu} \delta v_{p_{1}} \\
& \tau \frac{d}{d t} \delta q_{1} \quad=\gamma^{\prime} \beta \delta q_{0}-\Delta \gamma^{\prime} \beta \delta p_{0}-\gamma^{\prime} \delta q_{1}+\Delta \gamma^{\prime} \delta p_{1}-\gamma^{\prime} \delta q_{2}-\Delta \gamma^{\prime} \delta p_{2}+ \\
& +\sqrt{2 \gamma} \delta u_{q_{1}}+\sqrt{2 \mu} \delta v_{q_{1}} \\
& \tau \frac{d}{d t} \delta p_{2} \quad=\gamma^{\prime} \beta \delta p_{0}+\Delta \gamma^{\prime} \beta \delta q_{0}+\gamma^{\prime} \delta p_{1}-\Delta \gamma^{\prime} \delta q_{1}-\gamma^{\prime} \delta p_{2}-\Delta \gamma^{\prime} \delta q_{2}+ \\
& +\sqrt{2 \gamma} \delta u_{p_{1}}+\sqrt{2 \mu} \delta v_{p_{1}} \\
& \tau \frac{d}{d t} \delta q_{2} \quad=\gamma^{\prime} \beta \delta q_{0}-\Delta \gamma^{\prime} \beta \delta p_{0}-\gamma^{\prime} \delta q_{1}-\Delta \gamma^{\prime} \delta p_{1}-\gamma^{\prime} \delta q_{2}+\Delta \gamma^{\prime} \delta p_{2}+ \\
& +\sqrt{2 \gamma} \delta u_{q_{1}}+\sqrt{2 \mu} \delta v_{q_{1}}
\end{aligned}
$$

onde foram utilizadas as expressões

$$
\delta u_{j}=\frac{e^{i \varphi_{j}}}{2}\left(\delta u_{p j}+i \delta u_{q j}\right) \quad \text { e } \delta v_{j}=\frac{e^{i \varphi_{j}}}{2}\left(\delta v_{p j}+i \delta v_{q j}\right)
$$

para as quadraturas dos modos de vácuo inseridos pelas transmissões dos espelhos e pelas perdas espúrias, respectivamente. Definimos também a variável $\beta=p / p_{0}$ e eliminamos a constante $\chi$ utilizando a equação (4.3.2).

Este sistema de equações é mais simples de ser analisado quando escrito na forma matricial e desenvolvido com o auxílio de programas de computação simbólica, tais como Maple e Mathematica. A seguir veremos o tratamento matricial.

\subsection{Solução Matricial}

Discutimos no capítulo 2 como é feita a análise das flutuações em uma frequência específica, denominada frequência de análise. Por conta disso, reescrevemos o conjunto de equações (4.4.5)-(4.4.10) no espaço de Fourier, utilizando as expressões (2.2.16) para as quadraturas amplitude e fase de forma que $d / d t \rightarrow i \Omega$.

Vamos definir os vetores que contém as flutuações das quadraturas dos campos:

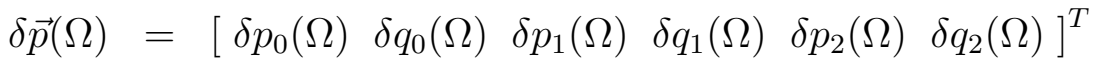

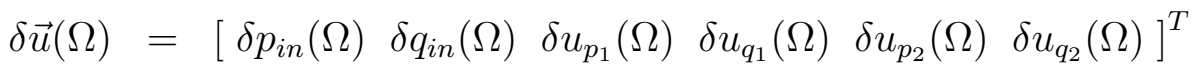

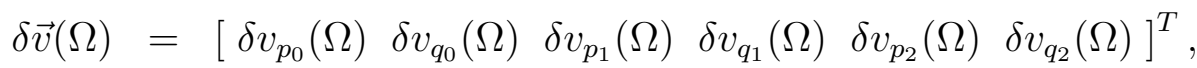


sendo $\delta \vec{p}, \delta \vec{u}$ e $\delta \vec{v}$ associados às flutuações das quadraturas dos campos de entrada, perdas por transmissão dos espelhos e perdas espúrias, respectivamente.

Com estes vetores o conjunto de equações (4.4.5)-(4.4.10) é compactado na equação matricial

$$
\underbrace{\left(-\mathbf{A}+2 i \Omega^{\prime} \gamma^{\prime} \mathbf{I}\right)}_{\mathbf{M}_{\mathbf{A}}\left(\boldsymbol{\Omega}^{\prime}\right)} \delta \vec{p}(\Omega)=\mathbf{T}_{\mathbf{u}} \delta \vec{u}(\Omega)+\mathbf{T}_{\mathbf{v}} \delta \vec{v}(\Omega),
$$

em que $\mathbf{I}$ é uma matriz identidade e $\mathbf{A}$ a matriz de arrasto

$$
\mathbf{A}=\left(\begin{array}{cccccc}
-\gamma_{0}^{\prime} & 0 & -\gamma^{\prime} \beta & 0 & -\gamma^{\prime} \beta & 0 \\
0 & -\gamma_{0}^{\prime} & 0 & -\gamma^{\prime} \beta & 0 & -\gamma^{\prime} \beta \\
\gamma^{\prime} \beta & 0 & -\gamma^{\prime} & 0 & \gamma^{\prime} & 0 \\
0 & \gamma^{\prime} \beta & 0 & -\gamma^{\prime} & 0 & -\gamma^{\prime} \\
\gamma^{\prime} \beta & 0 & \gamma^{\prime} & 0 & -\gamma^{\prime} & 0 \\
0 & \gamma^{\prime} \beta & 0 & -\gamma^{\prime} & 0 & -\gamma^{\prime}
\end{array}\right)
$$

obtida para dessintonias nulas, o que é justificado por trabalharmos em nosso experimento com OPO travado na ressonância com os três campos. Os vetores $\mathbf{T}_{\mathbf{u}}$ e $\mathbf{T}_{\mathbf{v}}$ tem em suas entradas os termos responsáveis pelas perdas devido a transmissão dos espelhos e perdas espúrias,

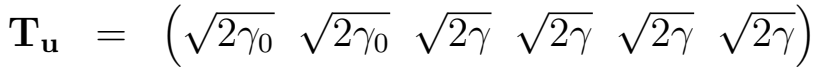

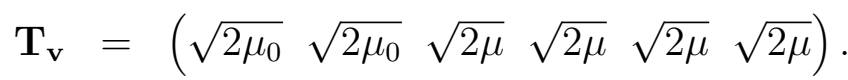

De acordo com o formalismo de entrada e saída na cavidade [Yurke 1984], escrevemos as quadraturas dos campos de saída do OPO,

$$
\delta \vec{p}_{\text {out }}(\Omega)=-\delta \vec{u}(\Omega)+\mathbf{T}_{\mathbf{u}} \delta \vec{p}(\Omega)
$$

de onde obtemos, com o auxílio da equação (4.5.4),

$$
\delta \vec{p}_{\text {out }}(\Omega)=\left(\mathbf{T}_{\mathbf{u}} \mathbf{M}_{\mathbf{A}}^{-\mathbf{1}}\left(\mathbf{\Omega}^{\prime}\right) \mathbf{T}_{\mathbf{u}}-\mathbf{I}\right) \delta \vec{u}+\mathbf{T}_{\mathbf{u}} \mathbf{M}_{\mathbf{A}}^{-\mathbf{1}}\left(\mathbf{\Omega}^{\prime}\right) \mathbf{T}_{\mathbf{u}} \delta \vec{v}
$$

Com esta última equação é possível calcular os espectros de ruído e correlações dos feixes de saída, dados pelas equações (2.4.29) e (2.7.41), que permitem construir a matriz de covariâncias do sistema $V_{O P O}$, definida como

$$
V_{\mathrm{OPO}}=S_{\mathrm{OPO}}=\operatorname{Re}\left[\left\langle\delta \vec{p}_{\text {out }}(\Omega) \delta \vec{p}_{\text {out }}^{T}(-\Omega)\right\rangle\right]
$$

Essa equação pode ainda ser desmembrada em matrizes de contribuições específicas

$$
V_{\mathrm{OPO}}=\mathbf{I}+\mathbf{V}_{\text {puro }}+\mathbf{V}_{\text {perdas }}
$$


O termo

$$
\begin{aligned}
\mathbf{V}_{\text {puro }}= & \mathbf{T}_{\mathbf{u}} \mathbf{M}_{\mathbf{A}}^{-1}\left(\Omega^{\prime}\right) \mathbf{T}_{\mathbf{u}} \mathbf{T}_{\mathbf{u}} \mathbf{M}_{\mathbf{A}}^{-1}\left(-\Omega^{\prime}\right)^{\mathbf{T}} \mathbf{T}_{\mathbf{u}}+ \\
& -\mathbf{T}_{\mathbf{u}} \mathbf{M}_{\mathbf{A}}^{-1}\left(\Omega^{\prime}\right) \mathbf{T}_{\mathbf{u}}-\mathbf{T}_{\mathbf{u}} \mathbf{M}_{\mathbf{A}}^{-1}\left(-\Omega^{\prime}\right)^{\mathbf{T}} \mathbf{T}_{\mathbf{u}}
\end{aligned}
$$

representa os ruídos dos campos e as correlações entre os mesmos na ausência de perdas espúrias, levadas em consideração através da matriz

$$
\mathbf{V}_{\text {perdas }}=\mathbf{T}_{\mathbf{u}} \mathbf{M}_{\mathbf{A}}^{-1}\left(\Omega^{\prime}\right) \mathbf{T}_{\mathbf{v}} \mathbf{T}_{\mathbf{v}} \mathbf{M}_{\mathbf{A}}^{-1}\left(-\Omega^{\prime}\right)^{\mathbf{T}} \mathbf{T}_{\mathbf{u}}
$$

Esta é a descrição usualmente encontrada no tratamento do OPO acima do limiar. Porém, foi recentemente investigado em nosso grupo a presença de um excesso de ruído na quadratura fase dos campos de saída, devido à interação dos mesmos com fônons presentes na rede cristalina do meio não-linear, o que torna necessária a adição de mais um termo na descrição. O tratamento detalhado é encontrado na referência [Coelho 2009a]. Aqui nos limitaremos em dizer que este excesso de ruído não afeta as amplitudes do campo, ficando restrito à quadratura fase. Por conta disso, podemos adicionar à equação (4.5.4) o vetor,

$$
\delta \vec{Q}(\Omega)=\left[\begin{array}{llllll}
0 & \delta Q_{0}(\Omega) & 0 & \delta Q_{1}(\Omega) & 0 & \delta Q_{2}(\Omega)
\end{array}\right]^{T},
$$

que, ao repetir o tratamento anterior, dá origem à matriz

$$
\mathbf{V}_{\text {fase }}=\mathbf{T}_{\mathbf{u}} \mathbf{M}_{\mathbf{A}}^{-1}\left(\Omega^{\prime}\right) \mathbf{V}_{\mathbf{Q}}(\Omega) \mathbf{M}_{\mathbf{A}}^{-1}\left(-\Omega^{\prime}\right)^{\mathbf{T}} \mathbf{T}_{\mathbf{u}}
$$

com $V_{Q}=\langle\vec{Q}(\Omega) \vec{Q}(-\Omega)\rangle$, cujos elementos são dados por

$$
\left\langle Q_{i}(\Omega) Q_{j}(-\Omega)\right\rangle=\eta_{i j} \sqrt{p_{i} p_{j}}, \quad \text { com } i, j=0,1,2 .
$$

A constante $\eta_{i j}$ depende do comprimento de onda dos campos e da permissividade do meio e é denominada coeficiente de acoplamento de ruído. Os valores de $\eta_{i j}$ devem ser obtidos experimentalmente. Vemos que o ruído devido aos fônons depende da potência dos campos intracavidade $\left(p_{i}\right.$ e $\left.p_{j}\right)$, o que justifica a primeira medida de emaranhamento no OPO acima do limiar ter sido obtida somente para potências de bombeio próximas ao limiar de oscilação [Villar 2005]. Mais tarde foi verificado que este ruído pode ter seus efeitos reduzidos através da diminuição da temperatura do cristal [Coelho 2009a]. Assim, este último termo completa a lista dos ingredientes necessários para descrever quantitativamente os ruídos e correlações dos campos em condições mais gerais de operação.

Por estarmos analisando o caso de dessintonias nulas $\left(\Delta=\Delta_{0}=0\right)$, a matriz de covariância possui o seguinte aspecto,

$$
V_{\mathrm{OPO}}=\left(\begin{array}{cccccc}
S_{p_{0}} & 0 & C_{p_{0} p_{1}} & 0 & C_{p_{0} p_{2}} & 0 \\
0 & S_{q_{0}} & 0 & C_{q_{0} q_{1}} & 0 & C_{q_{0} q_{2}} \\
C_{p_{0} p_{1}} & 0 & S_{p_{1}} & 0 & C_{p_{1} p_{2}} & 0 \\
0 & C_{q_{0} q_{1}} & 0 & S_{q_{1}} & 0 & C_{q_{1} q_{2}} \\
C_{p_{0} p_{2}} & 0 & C_{p_{1} p_{2}} & 0 & S_{p_{2}} & 0 \\
0 & C_{q_{0} q_{2}} & 0 & C_{q_{1} q_{2}} & 0 & S_{q_{2}}
\end{array}\right)
$$


Os elementos da diagonal principal fornecem os ruídos das quadraturas dos feixes de saída, enquanto os termos fora dela nos dão as correlações entre pares de feixes. Por termos considerado dessintonias nulas, os elementos correspondentes às correlações entre amplitude e fase dos campos, $C_{p_{i} q_{j}}(i, j \in\{0,1,2\})$, são nulos. Após obter a matriz de covariâncias, torna-se relativamente fácil aplicar o critério PPT de Simon [Simon 2000] para verificar a existência de emaranhamento tripartite em nosso sistema.

\subsubsection{Aplicação do critério PPT}

Na seção (3.7.3), mostramos que a transposição parcial aplicada na matriz densidade de um sistema composto é traduzida como uma simples mudança de sinal em uma das variáveis do espaço de fase da função de Wigner. De modo específico, transpor um subsistema $j$ é equivalente a trocar o sinal da quadratura $q_{j}$.

Nesta tese, estudamos um sistema composto de três partes produzido pelo OPO acima do limiar: feixes bombeio refletido, sinal e complementar. Identificaremos a presença de emaranhamento no sistema tripartite testando as formas biseparáveis

$$
\begin{aligned}
\rho & =\sum_{j} p_{n}^{(0 \mid 12)} \rho_{n}^{(0)} \otimes \rho_{n}^{(1,2)}, \\
\rho & =\sum_{j} p_{n}^{(1 \mid 02)} \rho_{n}^{(1)} \otimes \rho_{n}^{(0,2)} \mathrm{e} \\
\rho & =\sum_{j} p_{n}^{(2 \mid 01)} \rho_{n}^{(2)} \otimes \rho_{n}^{(0,1)},
\end{aligned}
$$

realizando a transposição parcial com relação ao subespaço de um dos feixes. O operador densidade $\hat{\rho}^{(i)}$ representa o sistema $i$, enquanto $\hat{\rho}^{(j, k)}$ se refere ao estado formado pelos sistemas $j$ e $k(i, j, k=0,1,2)$.

Como exemplo, consideremos a transposição parcial com relação ao subespaço do feixe sinal. Isso equivale a trocar o sinal da quadratura $q_{1}$, alterando o sinal dos termos $C_{q_{0} q_{1}} \mathrm{e}$ $C_{q_{1} q_{2}}$ da matriz de covariância $V_{O P O}$. Após isso, calculamos o menor autovalor simplético seguindo o procedimento apresentado em 3.7.3. Rotularemos por $\nu_{j}$ o menor autovalor da matriz obtida pela transposição parcial do sistema $j$.

Na figura 4.3 apresentamos o autovalores simpléticos obtidos para diferentes transposições parciais como função da potência de bombeio relativa ao limiar de oscilação $(\sigma)$. Consideramos a frequência de análise normalizada pela largura de banda da cavidade $\Omega^{\prime}=0.5$ e supomos o OPO em condições ideais de operação, livre de perdas espúrias. Observamos a presença de emaranhamento em todas as bipartições possíveis ${ }^{3}$.

O autovalor simplético $\nu_{1}<1$ atesta o emaranhamento entre o feixe sinal e o subsistema composto pelo feixe complementar e bombeio refletido. Já $\nu_{0}$ mostra a existência

\footnotetext{
${ }^{3}$ Como consideramos perdas iguais para os feixes sinal e complementar, existe perfeita simetria entre os dois, o que implica $\nu_{1}=\nu_{2}$.
} 
de emaranhamento entre o feixe de bombeio refletido e o sistema composto pelos feixes gêmeos.

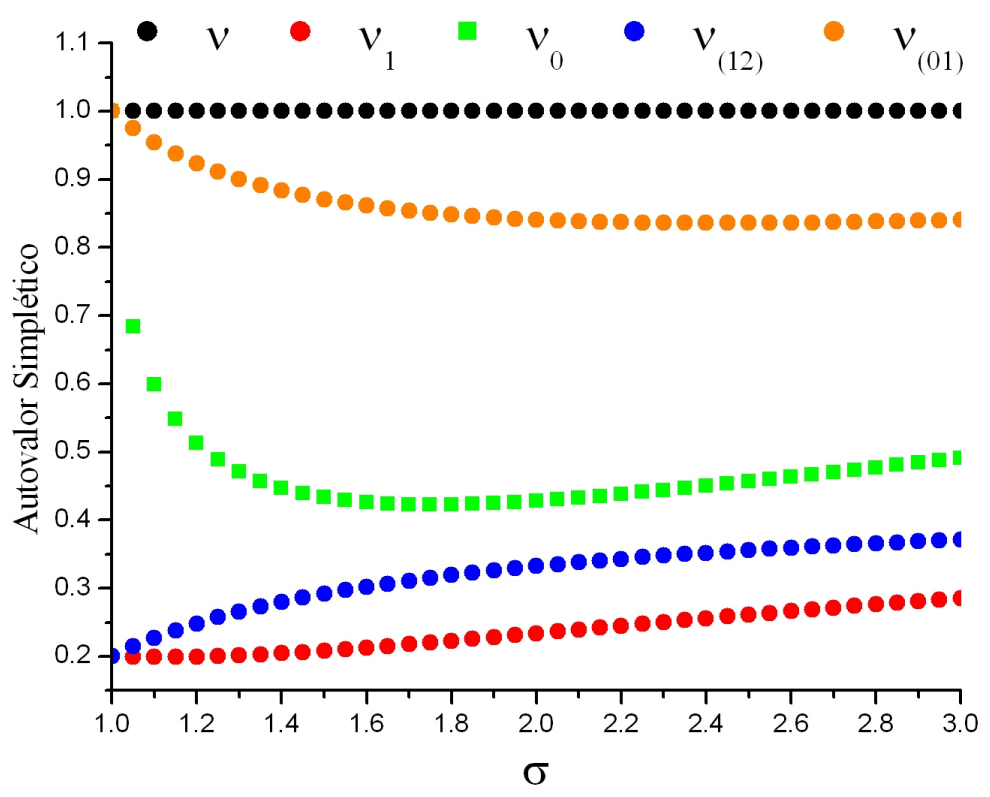

Figura 4.3: Menores autovalores simpléticos $\nu_{j}$ obtidos pela transposição do subsistema $j$ em função da potência de bombeio relativa ao limiar. Existe emaranhamento entre o feixe $j$ e o subsistema composto pelos outros dois quando $\nu_{j}<1$. A transposição dos feixes bombeio e sinal fornecem os autovalores $\nu_{0}$ (quadrados) e $\nu_{1}$ (círculos vermelhos), respectivamente. Realizando a transposição nos subespaços dos feixes gêmeos obtemos $\nu_{(12)}$ (círculos azuis), e para o subespaço formado por bombeio mais sinal obtemos $\nu_{(01)}$ (círculos laranjas). O autovalor $\nu$ é obtido sem a realização da transposição parcial. Adotamos, $\gamma_{0}=0,05, \gamma=0.002$ e $\Omega^{\prime}=0,5$.

É importante observar que ao considerar apenas o subespaço composto por dois campos os autovalores simpléticos apresentam um violação menor quando comparada com a obtida pelo sistema mais complexo formado pelos três feixes. Para o subespaço formado pelos feixes gêmeos obtemos o autovalor simplético $\nu_{(12)}$ referente à troca de sinal de $q_{1}$ ou $q_{2}$. Novamente, vemos que $\nu_{(12)}$ é maior que $\nu_{1}$ para todos os valores de $\sigma$, indicando que o emaranhamento entre os feixes gêmeos é mais expressivo quando incluímos as informações sobre o terceiro feixe. O autovalor $\nu_{(01)}$ também atesta o emaranhamento entre os feixes bombeio refletido e sinal, porém com valores próximos a 1. Essa análise é comprovada nas referências [Cassemiro 2008, Cassemiro 2007] que demonstram experimentalmente que a existência de correlações com o terceiro campo leva a uma correção das variâncias envolvidas no critério de van Loock e Furusawa [van Loock 2003]. Portanto, o emaranhamento é mais evidente quando consideramos o sistema global, formado pelos três feixes conjuntamente.

Por fim, calculamos os autovalores simpléticos da matriz de covariância na sua forma 
original, ou seja, sem realizar a transposição parcial em qualquer subsistema. O menor autovalor $\nu$ permanece constante e igual a 1 para toda faixa de potência de bombeio. Este é um resultado esperado, dado que os estados gerados pelo OPO na ausência de perdas espúrias $\left(\mu=\mu_{0}=0\right)$ são idealmente puros. A maneira mais simples de entender este resultado talvez esteja na análise da pureza, calculada a partir da expressão [Adesso 2006, Villar 2007b]

$$
\mathcal{P}=\frac{1}{\sqrt{\left|V_{O P O}\right|}},
$$

sendo $\left|V_{O P O}\right|$ o determinante da matriz de covariância, que deve ser igual a um se o sistema for puro. Portanto, a matriz de covariâncias de um sistema puro tripartite pode ser obtida a partir da matriz que representa três modos de vácuo independentes, uma matriz identidade $I$ de dimensão $6 \times 6$, dado que o determinante é invariante por transformações simpléticas.

Contudo, em uma situação física real os campos produzidos pelo OPO estão sempre sujeitos às perdas, sejam elas espúrias inerentes ao sistema ou causadas por elementos óticos existentes no caminho percorrido até os detectores. De um modo geral, elas são modeladas por divisores de feixes independentes colocados no caminho ótico. Cada divisor adiciona modos de vácuo ao modo do campo, tal como um reservatório à temperatura nula.

Atenuações provocadas por canais gaussianos transformam os operadores dos campos da seguinte forma [Holevo 2001]

$$
\hat{a}_{j} \longrightarrow \hat{a}_{j}^{\prime}=\sqrt{T_{j}} \hat{a}_{j}+\sqrt{1-T_{j}} \hat{a}_{j}^{(R)}
$$

onde $T_{j}$ é transmissão do divisor de feixes e $\hat{a}_{j}^{(R)}$ o operador associado aos modos do reservatório. Com essa transformação, a matriz de covariâncias passa a ser reescrita como

$$
V^{\prime}=\mathcal{L}(V)=\mathcal{L}(V-I) \mathcal{L}+I
$$

onde $\mathcal{L}=\operatorname{diag}\left(\sqrt{T_{0}}, \sqrt{T_{0}}, \sqrt{T_{1}}, \sqrt{T_{1}}, \sqrt{T_{2}}, \sqrt{T_{2}}\right)$ é a matriz que modela as perdas e $I$ uma matriz identidade de dimensão 6. Note que quando todas as transmissões se tornam nulas, a matriz de covariâncias se torna $V^{\prime}=I$, indicando que nessas circunstâncias serão medidos somente modos de vácuo.

Podemos então verificar como as perdas alteram o emaranhamento estudando seu efeito sobre os autovalores simpléticos, algo feito de modo empírico em nosso laboratório para o sistema tripartite. Medimos a matriz de covariâncias para os campos sem atenuação e, de modo controlado, adicionamos perdas com o uso de divisores de feixes, calculando para cada matriz de covariâncias resultante o autovalor simplético. Para sistemas bipartites foi feito um estudo analítico detalhado, apresentado na tese de outro estudante do grupo, Felippe A. S. Barbosa. Este estudo permitiu identificar diferentes 
classes de estados emaranhados, organizadas de acordo com a resposta do emaranhamento quando submetido às perdas.

Esta análise só faz sentido se investigarmos o emaranhamento com um critério necessário e suficiente, características presentes no PPT quando os estados analisados são gaussianos. Por conta disso, é necessário verificar se os campos produzidos pelo OPO satisfazem essa condição, que pode ser verificada através dos valores dos momentos de ordem superior das quadraturas dos campos. Trataremos deste assunto na próxima seção.

\subsection{Gaussianidade dos feixes produzidos pelo OPO acima do limiar}

Para verificar a existência de emaranhamento entre os campos bombeio refletido, sinal e complementar produzidos pelo oscilador paramétrico ótico (OPO) construído em nosso laboratório, utilizamos o critério de Positividade sob Transposição Parcial [Peres 1996, Simon 2000]. Para estados Gaussianos, o critério PPT torna-se necessário e suficiente para todas as decomposições do tipo $1 \times(N-1)$ [Werner 2001] onde $N$ é o número total de sistemas emaranhados. Em nosso sistema analisamos partições do tipo $1 \times 2$.

Porém existe uma pergunta bastante importante: Os três campos produzidos pelo OPO são Gaussianos? Muitos questionamentos têm surgido a este respeito, principalmente pelo fato do sistema não ser descrito por uma hamiltoniana quadrática. Estados comprimidos da luz são essenciais para geração de emaranhamento e são produzidos através de efeitos óticos não-lineares. Um exemplo bastante conhecido é a amplificação paramétrica degenerada, descrita por uma hamiltoniana quadrática nos operadores de criação e aniquilação. Para essa hamiltoniana, mostra-se que a função de Wigner tem um perfil gaussiano em termos das quadraturas do campo [Braunstein 2005].

Em se tratando de OPO acima do limiar algumas discussões e análises foram feitas, porém sem levar em consideração a difusão de fases dos campos produzidos [Dechoum 2010]. A fim de justificar nossa afirmação sobre a gaussianidade dos estados produzidos pelo nosso OPO, partimos de conceitos estatísticos simples. A função de Wigner para estados Gaussianos multimodos é dada por uma distribuição gaussiana [Simon 2000]

$$
W(\xi)=\frac{1}{\pi^{N} \sqrt{\operatorname{det}(V)}} \exp \left(\frac{-1}{2} \xi V \xi^{T}\right),
$$

onde os vetores de dimensão $2 N, \xi$, contêm as quadraturas amplitude e fase dos $N$ modos,

$$
\xi=\left(p_{1}, q_{1}, p_{2}, q_{2}, \ldots, p_{N}, q_{N}\right), \hat{\xi}=\left(\hat{p}_{1}, \hat{q}_{1}, \hat{p}_{2}, \hat{q}_{2}, \ldots, \hat{p}_{N}, \hat{q}_{N}\right) .
$$

A matrix $V$ é a matriz de covariâncias definida em (3.7.13).

É bem conhecido que uma distribuição Gaussiana é completamente caracterizada pelos momentos de primeira e segunda ordem. Em outras palavras, diz-se que os cumulantes 
de ordem superior a dois são nulos [Hofmann 2010]. Seguindo o tratamento encontrado nesta referência, vamos considerar a função característica $G(k)$ associada à distribuição $P(x)$ que define a probabilidade de uma variável aleatória $X$ assumir o valor $x$. A função $G(k)$ é definida como o valor médio de uma exponencial,

$$
G(k)=\left\langle e^{i k X}\right\rangle=\int d x e^{i k x} P(x),
$$

que ao ser expandida é reescrita como,

$$
G(k)=\sum_{n=0}^{\infty} \frac{(i k)^{n}}{n !}\left\langle X^{n}\right\rangle \quad \text { com } \quad\left\langle X^{n}\right\rangle=\int d x x^{n} P(x)
$$

onde é suposto que todos os momentos existem. A equação acima mostra que todos os momentos podem ser calculados a partir das derivadas da função característica,

$$
\left\langle X^{n}\right\rangle=\left.(-i)^{n} \frac{d^{n}}{d k^{n}} G(k)\right|_{k=0} .
$$

No entanto, se não conhecemos a função $G(k)$, é necessário obter informações sobre os momentos de modo alternativo.

Para uma distribuição gaussiana do tipo $p(x)=\left(2 \pi \sigma^{2}\right)^{-1 / 2} \exp \left[-\left(x-x_{0}\right)^{2} / 2 \sigma^{2}\right]$, onde $\langle X\rangle=x_{0}$ e $\left\langle\left(X-x_{0}\right)^{2}\right\rangle=\sigma^{2}$, é simples mostrar que a função característica é dada por $\phi(k)=e^{i k x_{0}} e^{-k \sigma^{2} / 2}$ e que, de fato, por meio da equação (4.6.5) obtemos os primeiro e segundo momentos, $\langle X\rangle=x_{0}$ e $\left\langle X^{2}\right\rangle=\sigma^{2}+x_{0}^{2}$. Além disso, podemos obter todos os momentos de ordem mais alta em termos dos dois primeiros.

Realizando a mudança de variável $X=x_{0}+\delta X$, com $\langle\delta X\rangle=0$, mostra-se que momentos de ordem ímpar $\left(\left\langle X^{2 n-1}\right\rangle\right)$ são nulos e os de ordem par se relacionam com o momento de segunda ordem através da expressão

$$
\left\langle X^{2 n}\right\rangle=(2 n-1) ! ! \sigma^{2 n}
$$

Este resultado nos mostra que a razão $\gamma \equiv\left\langle X^{2 n}\right\rangle /\left(\sigma^{2 n}\right)$ é um número muito bem definido igual a $(2 n-1)$ !! no caso de estados gaussianos.

Em um caso mais geral, onde temos um conjunto de $m$ variáveis aleatórias $X=$ $\left(X_{1}, X_{2}, \ldots, X_{m}\right)$, as variáveis $x$ e $k$ na equação (4.6.3) são substituídas por vetores $x=$ $\left(x_{1}, x_{2}, \ldots, x_{m}\right)$ e $k=\left(k_{1}, k_{2}, \ldots, k_{m}\right)$, e o momento de enésima ordem dado por,

$$
\left\langle X_{1}^{n_{1}} X_{2}^{n_{2}} \ldots X_{m}^{n_{m}}\right\rangle=(-i)^{N} \frac{\partial^{N}}{\partial k_{1}^{n_{1}} \partial k_{2}^{n_{2}} \ldots \partial k_{m}^{n_{m}}} \phi(k),
$$

$\operatorname{com} N=\sum_{i}^{m} n_{i}$

Para a equação (4.6.1), que pode ser considerada uma distribuição gaussiana com valor médio nulo, obtemos a função característica $\phi=e^{\left(k^{T} \cdot V \cdot k\right) / 2}$, de onde vemos que os momentos dependem somente dos termos $V_{i j}=\left\langle X_{i} X_{j}\right\rangle$ da matriz de covariâncias. De 
acordo com a expressão (4.6.7), os momentos de ordem 3 são nulos e os de ordem 4 dados por

$$
V_{i j k l}=V_{i j} V_{k l}+V_{i k} V_{j l}+V_{j k} V_{i l} .
$$

Usamos essa análise para estudar a gaussianidade dos campos produzidos pelo OPO. A partir das flutuações medidas, calculamos os momentos de ordem superior e comparamos os resultados obtidos com os valores esperados para uma distribuição gaussiana. Se os valores coincidirem, as duas distribuições são idênticas [Krauth 1998]. Outro método estatístico pode ainda ser utilizado, o teste de hipótese nula. A ideia se baseia no cálculo de um parâmetro denominado $p$-valor que permite descartar ou confirmar a gaussianidade de uma dada distribuição. Este teste foi aplicado na investigação do caráter gaussiano dos estados bipartite formados pelos feixes gêmeos produzidos por um OPO operando abaixo do limiar de oscilação [Auria 2010].

Os resultados obtidos serão apresentados no capítulo seguinte e mostram que em termos práticos os estados produzidos são de fato gaussianos.

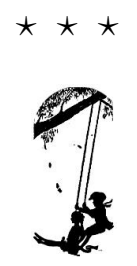

William B. Case and Mark A. Swanson, The pumping of a swing from the seated position, Am. J. Phys. 58, 463 (1990). William B. Case, The pumping of a swing from the standing position, Am. J. Phys. 64, 215 (1996).

Neste capítulo, apresentamos o Oscilador Paramétrico Ótico, nossa fonte de campos emaranhados. Este dispositivo é formado por um cristal de suscetibilidade não-linear disposto no interior de uma cavidade ótica. O cristal aniquila fótons do feixe de bombeio e gera pares de fótons que constituem os feixes sinal e complementar. Após a descrição teórica, a matriz de covariâncias foi apresentada com a subsequente aplicação do critério PPT em estados bipartite e triparte. A seguir apresentaremos os resultados experimentais inéditos obtidos neste trabalho de tese. 


\title{
Capítulo 5
}

\section{Resultados Experimentais}

\author{
Queremos saber, quando vamos ter \\ Raio laser mais barato \\ Queremos de fato um relato \\ Retrato mais sério do mistério da luz...
}

G. Gil (1976)

Os diversos avanços na área de Informação Quântica envolvendo processos de manipulação, armazenamento e comunicação englobam diferentes sistemas físicos, tais como átomos individuais, pontos quânticos e íons aprisionados. Em uma estrutura mais complexa, pode-se pensar na criação de uma rede de informação quântica onde os sítios são ocupados por tais sistemas, sendo a luz o principal recurso de comunicação. Contudo, cada sistema possui uma frequência de ressonância característica, fato que levará à produção de feixes de luz emaranhados com comprimentos de onda distintos a ocupar uma posição de destaque na criação dessas redes. Mesmo em uma situação um pouco "mais simple", na qual os sítios são ocupados por átomos do mesmo tipo, é preciso gerar estados emaranhados da luz que contenham frequências de transmissão típicas de fibras óticas e outras correspondentes às transições atômicas.

Motivados por essa perspectiva, o Laboratório de Manipulação Coerente de Átomos e Luz (LMCAL) vem se concentrando em duas linhas de pesquisa, átomos frios e campos emaranhados, que podem ser conectadas em futuro próximo, possibilitando a troca de informação entre diferentes sistemas, como numa rede de informação. Um dos trabalhos de destaque do grupo foi a demonstração teórica da existência de emaranhamento entre os três campos, com diferentes comprimentos de onda, produzidos por um OPO triplamente ressonante acima do limiar de oscilação [Villar 2006]. Segundo o tratamento teórico, os feixes sinal e complementar deveriam apresentar correlações quânticas com o feixe bombeio de modo a causar violação das desigualdades de van Loock e Furusawa [van Loock 2003], escritas em termos das variâncias de combinações das quadraturas dos 
campos. De certa maneira, é compreensível que existam tais tipos de correlação, pois no processo de conversão paramétrica existe um acoplamento entre as intensidades dos campos, além de uma relação de fase bem definida entre os mesmos, vínculos impostos pelas relações de acordo de fase $\vec{k}_{0}=\vec{k}_{1}+\vec{k}_{2}$ e conservação de energia $\left(\omega_{0}=\omega_{1}+\omega_{2}\right)$. De fato, a compressão de ruído no feixe de bombeio refletido já havia sido observada experimentalmente por Kasai et al. [Kasai 1997] seguida da demonstração de compressão de ruído de fase, realizada por [Zhang 2001].

Uma vez prevista a existência de emaranhamento tripartite, a busca pela demonstração experimental do fenômeno teve seu início. Cassemiro et al. [Cassemiro 2007] observaram pela primeira vez a presença de correlações quânticas entre a soma das fases dos feixes gêmeos e a amplitude do feixe de bombeio refletido, mostrando em seguida que esta última também está correlacionada com a amplitude dos campos sinal e complementar. Em princípio, esses seriam os ingredientes para a verificação do emaranhamento multipartite (mais de dois subsistemas), porém, devido a um excesso de ruído espúrio que tem sua origem na interação dos campos com o cristal não-linear, as correlações quânticas observadas não foram suficientes para a verificação do emaranhamento. Foi justamente nesse ponto que iniciei meu trabalho no OPO, participando dos experimentos que demonstraram que o excesso de ruído se deve à interação com fônons [César 2008b] e, portanto, pode ter seus efeitos diminuídos ao reduzir a temperatura do cristal.

O trabalho apresentado nesta tese se encaixa neste contexto. Demonstramos, no período de mestrado [Coelho 2009a], que o ruído da quadratura fase dos campos diminuía consideravelmente quando o cristal não-linear era resfriado. Neste mesmo período, realizamos medidas preliminares de emaranhamento tripartite obtidas mantendo o cristal a $-10{ }^{\circ} \mathrm{C}$. Neste trabalho, despendemos mais esforços na busca de temperaturas mais baixas, hoje no entorno de $-25^{\circ} \mathrm{C}$ em regime estável de operação. Analisando as flutuações das quadraturas amplitude $(\hat{p})$ e fase $(\hat{q})$ dos três campos produzidos pelo OPO, juntamente com as correlações existentes entre essas grandezas, obtivemos a primeira medida de emaranhamento tripartite produzido por única fonte, sendo a diferença entre os comprimentos de onda dos feixes a principal característica do sistema.

Utilizamos o critério de Positividade sob Transposição Parcial (PPT) para identificar o emaranhamento em nosso sistema. Para aplicá-lo, medimos os momentos de segunda ordem das quadraturas do campo, organizando-os em uma matriz de covariâncias que, para estados gaussianos, carrega toda informação disponível. Apresentamos aqui como as medidas são realizadas, os resultados obtidos e as modificações no aparato experimental que possibilitaram investigar a dinâmica do emaranhamento quando os campos estão sujeitos a atenuações.

Em seguida, realizamos o estudo experimental da dinâmica do emaranhamento medido frente a perdas, mostrando a existência de regiões de parâmetros para os quais o emaranhamento se perde para perdas finitas [Coelho 2009b]. Analisamos também o caso 
mais simples, o sistema bipartite, constituído pelos feixes gêmeos sinal e complementar [Barbosa 2010a]. A fim de justificar o uso do critério PPT como sendo necessário e suficiente para identificar o emaranhamento em nosso sistema, estudamos a estatística gaussiana das flutuações dos campos.

\subsection{Experimento}

Nosso aparato experimental mantém sua estrutura básica há pelo menos sete anos, sendo também descrito em detalhe em dissertações [César 2008, Coelho 2009a] e teses anteriores do grupo [Villar 2007b, Cassemiro 2008]. Ao longo deste período, novos elementos foram adicionados ao experimento e partes originais foram bastante melhoradas, conforme será descrito no decorrer do texto. A figura 5.1 apresenta o conjunto dos principais elementos encontrados em nosso experimento.

Utilizamos um OPO bombeado pelo segundo harmônico (comprimento de onda igual a $532 \mathrm{~nm}$ ) de um laser Nd:YAG (Diabolo), da empresa alemã Innolight GmbH. Em condições ideais de operação, este equipamento possui um laser de saída em $532 \mathrm{~nm}$ com até $900 \mathrm{~mW}$ de potência e largura de linha de cerca de $1 \mathrm{kHz}$. Uma saída auxiliar fornece um feixe laser em $1064 \mathrm{~nm}$ com $250 \mathrm{~mW}$, bastante útil para o alinhamento e caracterização de dispositivos óticos.

As quadraturas fase e amplitude do feixe de bombeio possuem excesso de ruído, característica que resulta na contaminação das quadraturas dos campos produzidos pelo OPO, encobrindo o emaranhamento existente. Este efeito foi também observado por outros dois grupos de pesquisas, um na França e outro nos Estados Unidos, liderados pelos Profs. Claude Fabre e Olivier Pfister, respectivamente. Por conta disso, realiza-se um processo de filtragem de ruído do campo, fazendo-o passar por uma cavidade ótica vazia de alta finesse, denominada cavidade de filtro [Willke 1998]. Após o filtro, os ruídos das quadraturas fase e amplitude passam a ser iguais aos de um estado coerente, quando analisados em frequências acima de $20 \mathrm{MHz}$ [Villar 2007b].

No que diz respeito à sua estrutura, a cavidade de filtro possui montagem em anel com comprimento igual a $L=1,15 \mathrm{~m}$, construída em uma base rígida com intuito de obter maior estabilidade, diminuindo as perturbações de origem mecânica. Para evitar contato direto com poeira e mantê-la limpa por mais tempo, a cavidade é revestida por acrílico. Quanto aos espelhos, foram usadas duas configurações: 


\section{Cavidade de filtro 1 (montagem original)}

Constituída de um espelho esférico côncavo, altamente refletor, com raio de curvatura $r=1 \mathrm{~m}$ e polarizadores para altas energias da Newport Corporation (modelo 11B00HP.6) que fazem o papel dos espelhos de entrada $(R=98,8(3) \%)$ e saída $(R=95,7(3) \%$ ). Com essas especificações a cavidade possui uma finesse $F=110$, largura de banda $\delta \nu=2,3(2) \mathrm{MHz}$, perdas espúrias iguais a $0,4(2) \%$ e transmissão máxima $T_{\max }=55 \%$.

Cavidade de filtro 2 (versão melhorada durante este trabalho de tese)

Espelho esférico côncavo, altamente refletor, com raio de curvatura $r=1 \mathrm{~m}$. Os polarizadores para altas energias foram trocados por espelhos de alta reflexão $R=99,1(1) \%$ com coating antirrefletor para $532 \mathrm{~nm}$ em incidência entre $43^{\circ}-45^{\circ}$, da empresa ATFilms. Com esta configuração a cavidade atual possui uma finesse $F=370$, largura de banda $\delta \nu=0,7(2) \mathrm{MHz}$, perdas espúrias iguais a $0,1(1) \%$ e transmissão máxima $T_{\max }=59 \%$.

Nas duas situações, a polarização do feixe é ajustada de modo a maximizar a transmissão da cavidade. A troca dos espelhos proporcionou uma finesse três vezes maior, com a consequente diminuição da largura de banda da cavidade, elevando sua capacidade de filtragem. Contudo, o estreitamento do seu sinal de transmissão leva a outras dificuldades técnicas, sendo a principal delas o travamento do seu comprimento na ressonância, feito por meio de um sistema de travamento eletrônico (lock-in). A ideia geral é modular a fase do laser com uma frequência específica e misturar um sinal de referência (reflexão ou transmissão da cavidade) com a modulação usada, a fim de obter um sinal discriminante que realimenta o PZT da cavidade, fixando seu comprimento na ressonância. As técnicas mais utilizadas são "Dither locking" e "Pound-Drever-Hall" (PDH), descritas de modo bastante detalhado nas referências [Ph77 Caltech, Black 2001].

Com relação ao OPO utilizado, este é formado por uma cavidade Fabry-Perot linear com espelhos de entrada e saída separados por uma distância $L=19,0(1) \mathrm{mm}$. O espelho de entrada possui refletibilidade de $70 \%$ para o feixe de bombeio (532 nm) e o de saída 96\% de reflexão para o infra-vermelho, ambos com raio de curvatura $r=50$ mm. O intervalo espectral livre, o mesmo para os três modos, é igual $\nu_{c}=5.1(1) \mathrm{GHz}$ e as finesses para o campo de bombeio, sinal e complementar são $F_{0}=18, F_{1}=135$, $F_{2}=115$, respectivamente. Esses valores são obtidos de forma direta e são importantes para verificar as previsões teóricas dos ruídos de quadratura dos campos produzidos, bem como avaliar o nível de compressão que pode ser obtido.

Utilizamos um cristal de KTP (potássio-titanil-fosfato, $\mathrm{KTiOPO}_{4}$ ) da empresa israelense Raicol Crystals Ltd com comprimento $\ell=12 \mathrm{~mm}$, índice de refração médio igual a 1,81(1) e coating antirrefletor para $532 \mathrm{~nm}$ e $1064 \mathrm{~nm}$. O casamento de fase deste cristal 


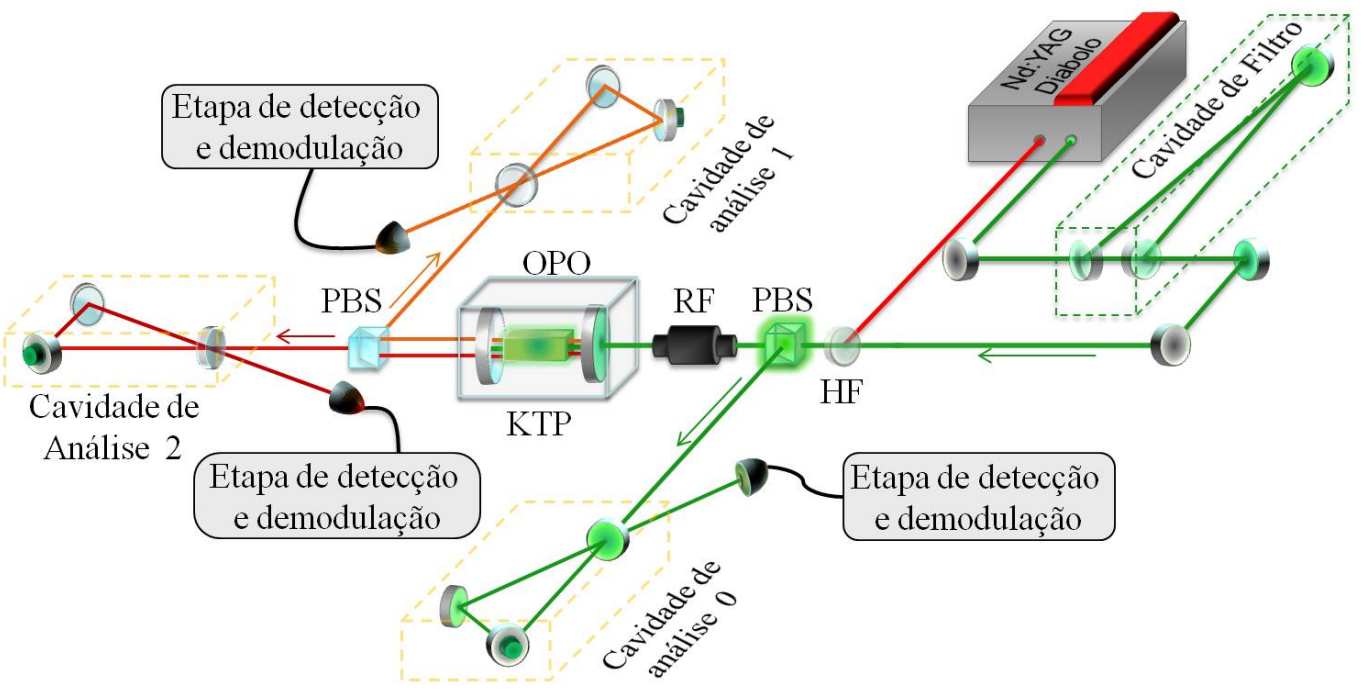

Figura 5.1: Esquema do aparato experimental. O laser Diabolo fornece duas saídas: feixe de bombeio em $532 \mathrm{~nm}$ (verde) e uma saída auxiliar em $1064 \mathrm{~nm}$ (representado em vermelho). O feixe de bombeio é filtrado e enviado para o OPO, mantido em uma caixa de vácuo para evitar condensação na superfície do cristal resfriado. No OPO há a produção dos feixes bombeio refletido, sinal e complementar, denotados pelos índices 0,1 e 2, respectivamente. Os comprimentos de onda dos campos são $\lambda_{0}=532.251 \mathrm{~nm}, \lambda_{1}=$ $1062.102 \mathrm{~nm}$, e $\lambda_{2}=1066.915 \mathrm{~nm}$. Sinal e complementar são separados por polarização em um PBS. Separa-se o feixe de bombeio refletido usando um rotator de Faraday (RF) e um PBS. Cada campo segue para sua cavidade de análise onde há a conversão das flutuações de fase em flutuações de amplitude. Após passarem pelas cavidades os campos seguem para a etapa de deteç̧ão e demodulação, cujos resultados são registrados em um computador.

é do tipo II: feixes sinal e complementar possuem polarizações ortogonais. Para que sejam fixadas as condições de casamento de fase e mantidos constantes os comprimentos de onda gerados, o cristal tem sua temperatura estabilizada por elementos Peltier ${ }^{1}$, controlados por um sistema eletrônico desenvolvido pelo grupo do Laboratoire Kastler Brossel em Paris (LKB). O controle ativo permite ajustar a temperatura dentro de uma faixa que se estende de $-25^{\circ} \mathrm{C}$ a $110^{\circ} \mathrm{C}$, com precisão de algumas dezenas de $\mathrm{mK}$ e tempo de resposta da ordem de um segundo, característica essencial para o travamento do OPO na ressonância.

Variar a temperatura do cristal dentro de uma faixa tão ampla é importante para caracterizar o comportamento do ruído de fase dos feixes produzidos. Além de filtrar o ruído do feixe de bombeio, é necessário diminuir a temperatura do cristal a fim de reduzir

\footnotetext{
${ }^{1}$ Para informações sobre o funcionamento dos elementos Peltier, ver http://www.heatsinkguide.com/peltier.htm.
} 
a interação dos fônons da rede cristalina com os campos, que tem como efeito o aumento de ruído de fase nos mesmos [César 2008b]. Para manter baixa a temperatura do cristal e evitar a condensação, precisamos colocar o OPO em vácuo. Para tanto, utilizamos uma caixa de alumínio conectada a uma bomba mecânica (modelo SD-40) da empresa Varian. O nível de vácuo produzido fica estável em torno de 7,6 $\times 10^{-3}$ mbar, suficiente para os nossos propósitos. Esta foi a primeira mudança significativa no OPO a partir de 2008, permitindo resfriar o cristal até $-25^{\circ} \mathrm{C}$ com o auxílio de um elemento Peltier de alta potência da empresa Melcor (modelo UT6-7-30F1) em contato com um reservatório resfriado por água proveniente de um chiller. Partes específicas dessa montagem estão ilustradas na figura 5.2.

O processo de resfriamento ocorre em três etapas: realização de vácuo produzido por uma bomba mecânica, refrigeração do reservatório através de circulação de água a aproximadamente $7{ }^{\circ} \mathrm{C}$ e resfriamento termoelétrico. Uma vez feito vácuo na caixa onde o OPO está inserido não há mais liberdade para alinhamento do mesmo, o que dificulta a otimização do alinhamento e consequente diminuição da potência de limiar. Ao final do processo, caso o cristal não esteja em uma posição adequada, é necessário refazer o procedimento. Uma nova montagem com maior liberdade deve ser preparada para trabalhos futuros.
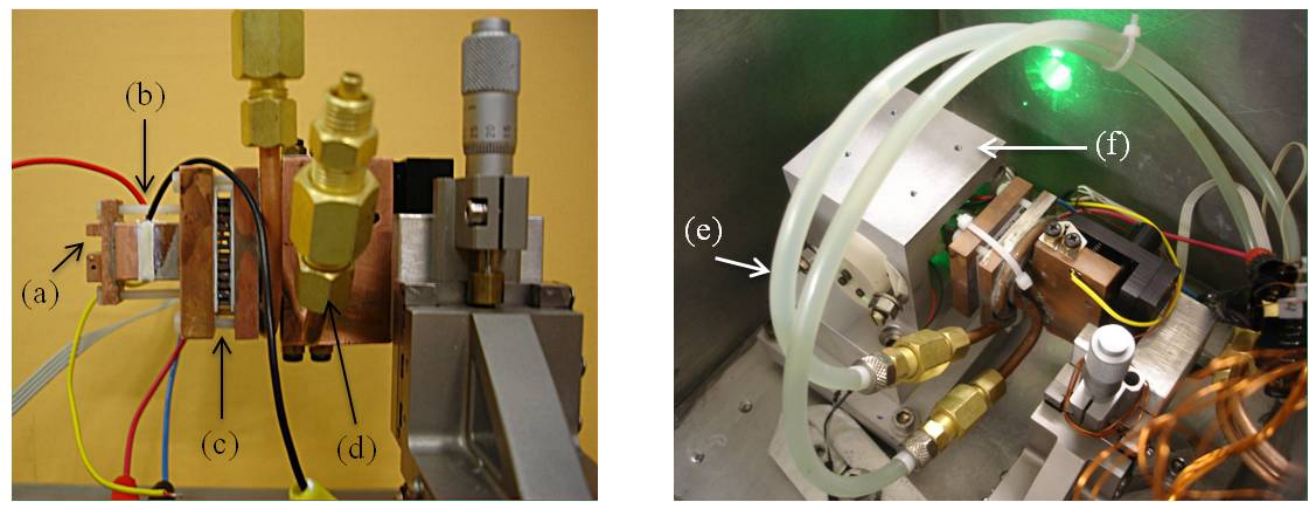

Figura 5.2: Suporte do cristal KTP. À esquerda temos: (a) local de fixação do cristal; (b) elemento Peltier de baixa potência controlado pelo sistema eletrônico desenvolvido no LKB, responsável pela estabilização de temperatura do cristal; (c) Peltier de alta potência - controlado por uma fonte de tensão comum - utilizado para gerar os maiores gradientes de temperatura; (d) duto por onde passa a água refrigerada. Na figura da direita ilustramos a montagem pronta para operação destacando em (e) os tubos que conectam o reservatório ao "chiller" e em (f) a montagem inteiriça de alumínio onde são fixados os espelhos que formam o OPO.

Os feixes sinal e complementar, separados por polarização, são enviados às suas respectivas cavidades de análise onde o ruído de fase é convertido, de forma controlada, em ruído de amplitude. Essas cavidades tem espelhos de acoplamento esférico com raio de 
curvatura $r=10 \mathrm{~cm}$ e reflexão $R=95 \%$ da empresa Innnolight $\mathrm{GmbH}$ e espelhos planos altamente refletores $R>99,8 \%$ (modelo 10QM20HM.15 da Newport). Com estas especificações, temos uma finesse $F=130$ e uma largura de banda $\delta \nu_{1,2} \approx 14(1) \mathrm{MHz}$, fornecendo uma rotação completa da elipse de ruído para frequência de análise mínima de $21 \mathrm{MHz}$.

O feixe de bombeio refletido é separado do feixe incidente utilizando um rotator de Faraday (RF) e um cubo divisor de feixes (PBS). Sua cavidade de análise possui espelho de acoplamento com raio de curvatura $r=10 \mathrm{~cm}$ e reflexão $R=96 \%$. Os demais espelhos, planos, possuem reflexão $R>99,8 \%$. Isso nos dá uma finesse $F=110$, largura de banda $\delta \nu_{0} \approx 12(1) \mathrm{MHz}$ e perdas espúrias de $4 \%$, praticamente causadas apenas por imperfeições no coating do espelho de entrada. A fração da intensidade do feixe refletido na ressonância das cavidades é $R_{\text {min }}=0,77(1)$ para os feixes gêmeos e $R_{\text {min }}=0,58(2)$ para o bombeio. A foto da montagem experimental é apresentada na figura 5.3.

Para garantir que as elipses de ruído dos campos sejam giradas de forma síncrona, as cavidades são construídas com características similares. Todas são praticamente inteiriças, constituídas de peças de alumínio fixadas umas às outras por parafusos, a fim de evitar ressonâncias mecânicas. A temperatura é mantida próxima à ambiente com precisão de $0,1^{\circ}$ C. Esses cuidados são necessários para diminuir o deslocamento dos sinais de transmissão durante o tempo de aquisição relativamente longo das medidas, da ordem de $750 \mathrm{~ms}$.

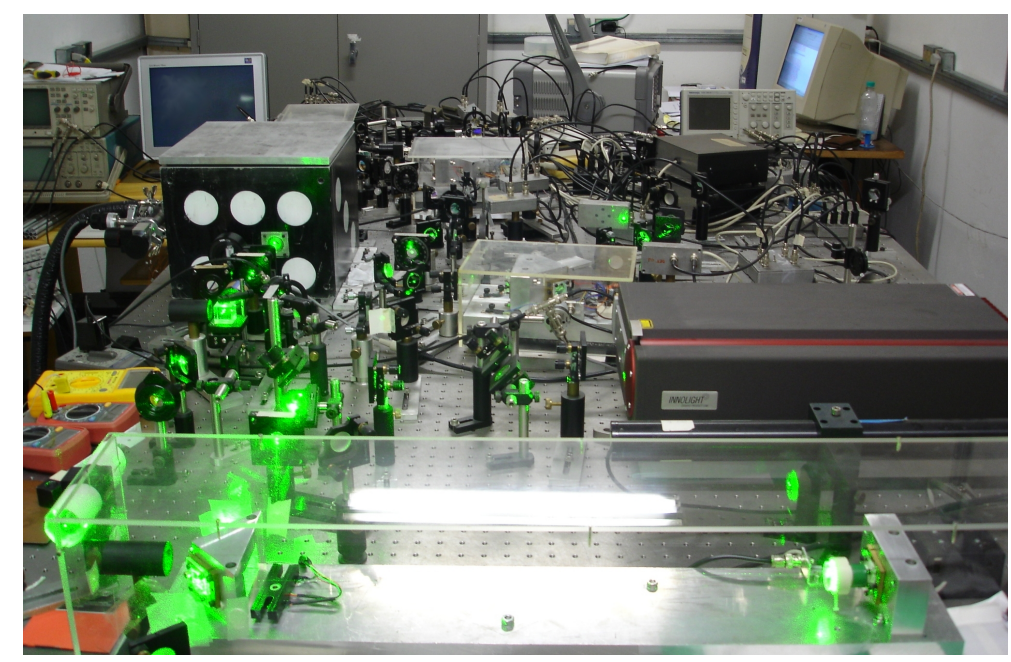

Figura 5.3: Foto da montagem na sua configuração atual. Na parte inferior, vemos a cavidade de filtro dentro de uma grande caixa de acrílico. À direita, o laser de bombeio Diabolo. As cavidades estão cobertas por pequenas caixas de acrílico, e o OPO, dentro da caixa de vácuo pintada de forma a reproduzir um dado preto com bolas brancas, arte primeiramente idealizada por A. S. Villar em referência à frase de Einstein "God does not play dice with the Universe".

As medidas de ruído dos três feixes são provenientes de fotodetectores que medem 
somente intensidade dos campos através de efeito fotoelétrico. Para o infravermelho são usados fotodiodos da marca Epitaxx ETX300 com eficiência de 95(2)\% e para o feixe de $532 \mathrm{~nm}$ usamos o modelo Hamamatsu S5973-02 com eficiência de 94(2)\%. As fotocorrentes produzidas são convertidas em voltagem ${ }^{2}$ por meio de um circuito de amplificação e separadas em duas componentes de frequência: componente DC, baixa frequência, e componente HF, alta frequência. Os sinais DC são as intensidades médias dos feixes, denotadas nos programas de análise por $\mathrm{DC} i(i=0,1$ e 2 , para bombeio refletido, sinal e complementar, respectivamente). A eficiência total de detecção, levando em consideração a passagem pela ótica envolvida, é de $87 \%$ para os feixes gêmeos e $65 \%$ para o bombeio.

Antes de serem adquiridos, os sinais HF (onde estão as flutuações quânticas das quadraturas) passam por duas importantes etapas do experimento. Primeiramente, um filtro ativo com largura de banda igual a $200 \mathrm{kHz}$ gera uma atenuação de $40 \mathrm{~dB}$ na componente de frequência igual a $12 \mathrm{MHz}$. A eletrônica do Diabolo utiliza um sinal periódico com esta frequência para travar a cavidade de geração do segundo harmônico em $532 \mathrm{~nm}$. Isto gera um ruído tão intenso em $12 \mathrm{MHz}$, que se faz necessário filtrar também seu segundo harmônico, em $24 \mathrm{MHz}$, a fim de evitar a saturação da eletrônica.

Após a filtragem, cada sinal é enviado a um demodulador onde é realizado o batimento com uma onda senoidal (sinal de referência) de frequência $\Omega=21 \mathrm{MHz}$, denominada frequência de análise. As componentes de baixa frequência (menores que $300 \mathrm{kHz}$ ) resultantes do batimento são denotadas $\mathrm{HF} i(i=0,1,2)$. Assim como a cavidade de filtro, o sistema de detecção sofreu alterações, descritas a seguir para um único campo, conforme ilustrado na figura 5.4, sendo o mesmo válido para os demais.

Sistema de detecção 1 (versão antiga)

Após ser refletido por sua cavidade de análise o feixe passa por um PBS onde é dividido em duas partes de intensidades iguais. Cada parte é direcionada para um fotodetector a fim de evitar saturação no regime de altas intensidades. As flutuações da fotocorrente geradas em cada detector são somadas eletronicamente e o resultado enviado a um canal de demodulação de onde obtemos o sinal HF correspondente à frequência de análise $\Omega$.

Sistema de deteç̧̃̃o 2 (versão melhorada durante este trabalho de tese)

Nesta configuração as flutuações da fotocorrente de cada detector são en-

\footnotetext{
${ }^{2}$ Alguns gráficos terão em seu eixo horizontal a grandeza DC em volts indicando a quantidade de luz que chega ao detector. Para converter para unidade de potência (Watt) basta lembrarmos da Lei de Ohm, $V=R I$. A corrente é definida como o fluxo de carga por unidade de tempo, $\mathrm{I}=e \Delta n_{e} / \Delta t \mathrm{e}$ a potência de cada elétron ejetado no semicondutor $P_{d e t}=\hbar \omega \Delta n_{e} / \Delta t$. Após manipulações algébricas simples, obtém-se $P_{d e t}=h c V /(e \lambda R)$, onde $\lambda$ é o comprimento de onda da luz que chega ao detector, $e$ a carga do elétron, $h$ constante de Planck e $c$ a velocidade da luz. A resistência tipicamente usada em nossos fotodetectores é $R=1 K \Omega$.
} 
viadas para um canal de demodulação e registradas individualmente. Após a demodulação são obtidos dois sinais, HFA e HFB, sendo a soma e a subtração realizadas após a aquisição.

A principal diferença entre as duas configurações está na forma como é calibrado o ruído quântico padrão (seção 2.6.1). Com a primeira, obtemos um coeficiente $\beta$, comum a toda série de medidas, que, ao ser multiplicado pela intensidade do campo, fornece o ruído de referência. A segunda, fornece a calibração do shot-noise ponto a ponto, aumentando a precisão dos ruídos medidos. Esta forma de detecção foi desenvolvida visando medir com maior precisão os ruídos das quadraturas amplitude e fase do campo, normalizados pelo shot-noise. Vemos a importância disto na medida de perda de emaranhamento entre os feixes gêmeos, por exemplo, que só foi possível com este "novo" método de calibração, pois as quantidades necessárias para verificar o fenômeno estavam restritas a uma pequena faixa de valores. Discutiremos as diferenças entre os dois sistemas de detecção nas duas próximas subseções.

Os dados são adquiridos pelo computador por meio de um programa em LabView, através da placa de aquisição NI-DAQ (modelo BNC-6110) da National Instruments, conectada a uma placa conversora analógico-digital (A/D) da mesma empresa (modelo PCI-2110). Cada medida possui um total de 450 mil pontos, lidos a uma taxa de 600 $\mathrm{kHz}$, determinada pela largura de banda do demodulador. Dessa forma, o intervalo de tempo de cada aquisição é aproximadamente igual a $750 \mathrm{~ms}$.

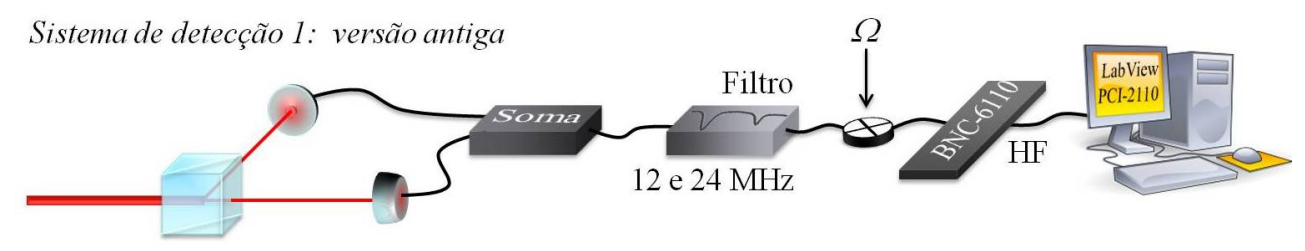

Sistema de detecção 2; versão melhorada

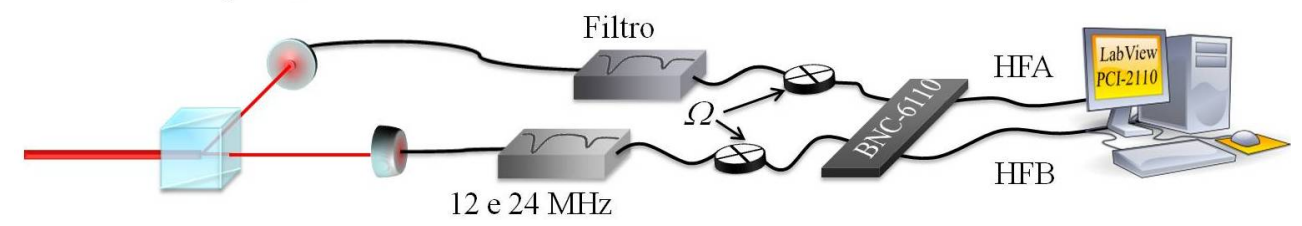

Figura 5.4: Sistema de deteç̧ão 1: as flutuações de fotocorrente dos detectores são somadas eletronicamente e suas componentes em 12 e $24 \mathrm{MHz}$ filtradas, seguindo para uma etapa de demodulação onde ocorre a mistura com um sinal periódico de frequência $\Omega$, que resultará em flutuações correspondentes a essa frequência específica. Por fim, os sinais são enviados para o computador onde serão adquiridos por um software em LabView. Sistema de detecção 2: são realizados os mesmos procedimentos anteriores, a menos da etapa de soma, realizada posteriormente via programa de análise.

O programa de análise dos dados, também em LabView, divide a série de 450 mil 
pontos em grupos de 1000, calculando para cada grupo o valor médio dos sinais DC $i$, a variância das flutuações $\mathrm{HF} i$ ( $\mathrm{HF} i \mathrm{~A}$ e $\mathrm{HF} i \mathrm{~B}$ ), correlações e ruídos da soma e subtração dos sinais medidos.

Devido à incerteza existente nos componentes eletrônicos (valores de resistência, capacitância, etc.), canais que recebem a mesma quantidade de luz podem ter uma resposta ligeiramente diferente. Como os campos são divididos em intensidades iguais, é completamente razoável que a amplitude do ruído seja a mesma. Por este motivo, é necessário calibrar e corrigir a diferença existente por meio da comparação dos sinais. Devemos novamente separar em duas etapas, correspondentes aos sistema de detecção 1 e 2. Vejamos:

\section{Calibração da resposta eletrônica 1 (versão antiga)}

Primeiramente, na ausência de luz, medimos a flutuação do sinal eletrônico $\mathrm{HFe}$ e calculamos a variância $\Delta^{2} \mathrm{HFe}$ para cada canal. Em seguida, realizamos medidas de flutuações para várias intensidades e calculamos os ruídos $\Delta^{2} \mathrm{HF}$, subtraindo posteriormente o ruído eletrônico medido. Para o infra-vermelho (IR), usamos a saída auxiliar em $1064 \mathrm{~nm}$ do Diabolo. O feixe é dividido em duas partes com mesma potência, uma enviada para a etapa de detecção do feixe sinal e a outra para a do feixe complementar, onde são feitas as medidas de flutuações para diferentes intensidades. Por fim, fazemos o gráfico $\Delta^{2} \mathrm{HF} 2 \times \Delta^{2} \mathrm{HF} 1$ de onde obtemos o coeficiente angular $g$, usado para compensar a diferença de resposta eletrônica entre os pares de detectores.

Calibração da resposta eletrônica 2 (nova versão)

Nesta nova configuração também realizamos a medida do ruído eletrônico e sua subtração dos sinais $\mathrm{HF} i \mathrm{~A}$ e $\operatorname{HF} i \mathrm{~B}(i=0,2,3)$. O cálculo do coeficiente $g$ é feito comparando a amplitude dos ruídos medidos por detectores de um mesmo par.

Após a medida, os ruídos são corrigidos levando em conta o parâmetro $g$ e em seguida normalizados pelo shot-noise. O procedimento para medida do ruído quântico padrão está exposto na seção 2.6.1. É neste ponto que surge a principal diferença entre as configurações 1 e 2, evidenciando a grande vantagem da segunda frente a primeira. As diferenças são discutidas a seguir.

\subsubsection{Calibração do shot-noise: Configuração 1}

Com a configuração antiga, a medida do shot-noise para os feixes sinal e complementar é realizada por meio de uma detecção balanceada usando feixe infravermelho do laser Diabolo. O feixe é dividido ao meio, sendo uma parte enviada para o par de detectores do feixe sinal e outra para o par correspondente ao feixe complementar. Após a aquisição, 
calculamos a subtração dos sinais provenientes de cada par e fazemos o gráfico da variância da subtração como função da intensidade total, $\langle D C 1+D C 2\rangle$ (figura 5.5(a)).

No que diz respeito ao feixe de bombeio, a medida de suas flutuações já fornece de forma direta o ruído quântico padrão pois, ao ser filtrado, tem suas flutuações de fase e amplitude reduzidas ao nível do shot-noise. Por conta disso, o gráfico $\Delta^{2} H F 0 \times\langle D C 0\rangle$ (figura 5.5(b)) nos dá informação suficiente para calibração do ruído quântico padrão. Das retas obtidas extraímos o coeficiente angular $\beta$ que, ao ser multiplicado por $\langle D C\rangle$, fornece o valor shot-noise para essa potência. Todos os ruídos são normalizados por esta quantidade, passando a ter o ruído quântico padrão como referência.

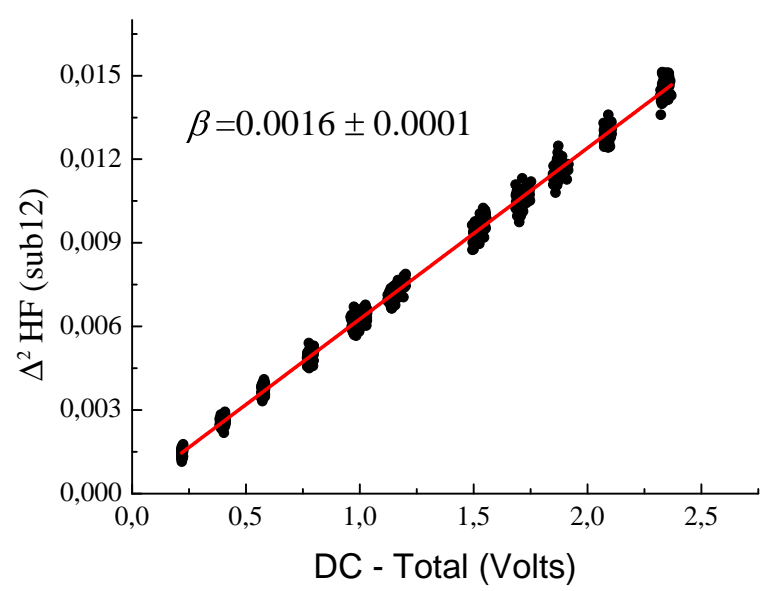

(a)

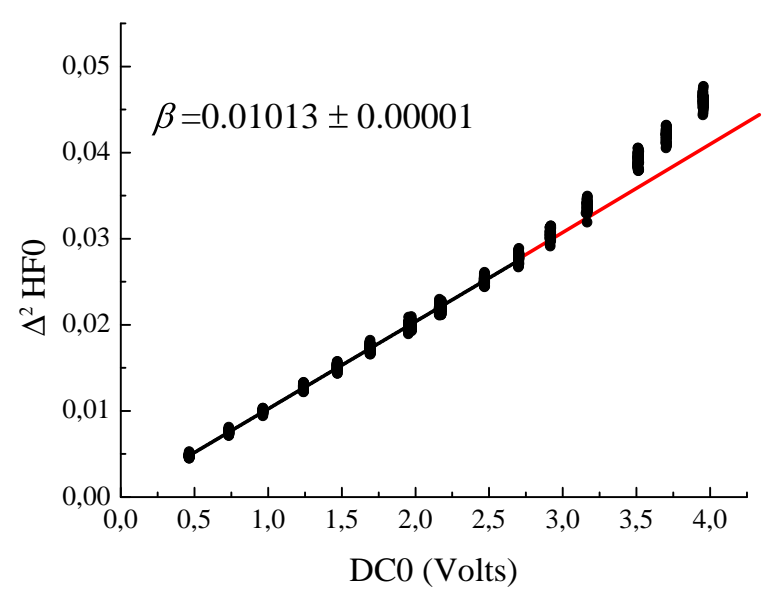

(b)

Figura 5.5: Calibração do shot-noise por meio do sistema de detecção 1. Em (a) apresentamos o gráfico do ruído da subtração dos sinais provenientes dos pares de detectores referentes aos feixes sinal e complementar como função de $\langle D C 1+D C 2\rangle$. O gráfico (b) é o ruído do feixe de bombeio como função de $\langle D C 0\rangle$. Os coeficientes $\beta$ são empregados na determinação do shot-noise. Em (b) a linearidade é observada somente para uma certa faixa de potência, fruto de uma provável saturação da eletrônica.

Uma observação importante deve ser feita. Vemos um comportamento não-linear para altas potências do feixe de bombeio. Isto sugere ou um excesso de ruído no feixe nesse regime ou uma saturação da eletrônica usada na detecção, sendo a pior hipótese a existência dos dois. Porém, como sabemos que um feixe coerente fornece um comportamento linear do ruído como função da intensidade, nos limitamos ao coeficiente $\beta$ obtido pelos pontos abaixo da linha vermelha desenhada no gráfico 5.5(b). Portanto, é importante fazer um diagnóstico mais detalhado da eletrônica que constitui nosso sistema de detecção. Este assunto é discutido a seguir. 


\section{Teste de sanidade da eletrônica}

Para nos certificarmos do bom funcionamento da eletrônica envolvida na detecção em nosso sistema, testes de sanidade foram realizados. Estes consistem em atenuar cada feixe produzido pelo OPO e analisar o comportamento das amplitudes dos ruídos das quadraturas e correlações quando os campos são submetidos a perdas. A atenuação foi feita com o uso de um divisor de feixes (beam splitter), conforme ilustrado na figura 2.4.

Sendo respectivamente $T=t^{2}$ e $R=r^{2}(R+T=1)$ a transmissão e reflexão do divisor de feixes, podemos escrever o modo de saída como $\hat{c}(\Omega)=t \hat{a}(\Omega)+r \hat{b}(\Omega)$ e escrever as flutuações do campo como

$$
\delta \hat{c}(\Omega)=t \delta \hat{a}(\Omega)+r \delta \hat{b}(\Omega)
$$

As flutuações de uma quadratura deste modo são definidas de acordo com a equação $(2.2 .10)$,

$$
\delta \hat{X}^{c}(\Omega)=\delta \hat{c}(\Omega) e^{-i \phi}+\delta \hat{c}^{\dagger}(-\Omega) e^{i \phi} .
$$

Substituindo (5.1.1) em (5.1.2), podemos calcular a variância de $\hat{X}_{i}^{c}(\mathrm{i}=0,1,2)$ e as correlações entre diferentes quadraturas dos feixes sinal, complementar e bombeio refletido, obtendo as expressões

$$
\begin{aligned}
\Delta^{2} X_{i}^{c}(\Omega) & =T_{i} \Delta^{2} X_{i}^{a}(\Omega)+\left(1-T_{i}\right) S Q L \quad \mathrm{e} \\
\left\langle\delta X_{i}^{c}(\Omega) \delta X_{j}^{c}(-\Omega)\right\rangle & =\sqrt{T_{i} T_{j}}\left\langle\delta X_{i}^{a}(\Omega) \delta X_{j}^{a}(-\Omega)\right\rangle,
\end{aligned}
$$

$(i, j=0,1,2$, com $i \neq j)$ que representam termos da matriz de covariâncias em função das atenuações aplicadas nos feixes. Para quantificar a atenuação do campo, a intensidade medida é normalizada pelo seu valor máximo (sem atenuação).

A equação (5.1.3) nos diz que o ruído de quadratura de um feixe deve diminuir de forma linear à medida que este é atenuado, indo ao nível de shot-noise (SQL) ${ }^{3}$ quando completamente bloqueado $\left(T_{i}=0\right)$. Este fato é facilmente compreendido quando consideramos que ao atenuar completamente o campo apenas modos de vácuo interagem com o detector. Ao normalizar as amplitudes de ruído pelo shot-noise, passamos a ter como referência o valor 1.0, uma vez que flutuações do estado de vácuo definem o ruído quântico padrão. As correlações entre as quadraturas, dadas pela equação (5.1.4) dependem das atenuações de cada campo, tendendo a zero quando um dos campos é completamente bloqueado $\left(T_{i}, T_{j} \rightarrow 0\right)$. Se a eletrônica do nosso sistema estiver funcionando corretamente as equações (5.1.3) e (5.1.4) devem ser satisfeitas pelos dados experimentais.

Realizamos este teste mantendo fixa a potência do feixe de bombeio e, por consequência, as potências dos feixes sinal e complementar. Submetemos à atenuação somente os dois últimos. Medimos os ruídos de fase e amplitude dos campos e as correlações entre

\footnotetext{
${ }^{3}$ Acrônimo de Standard Quantum Limit.
} 


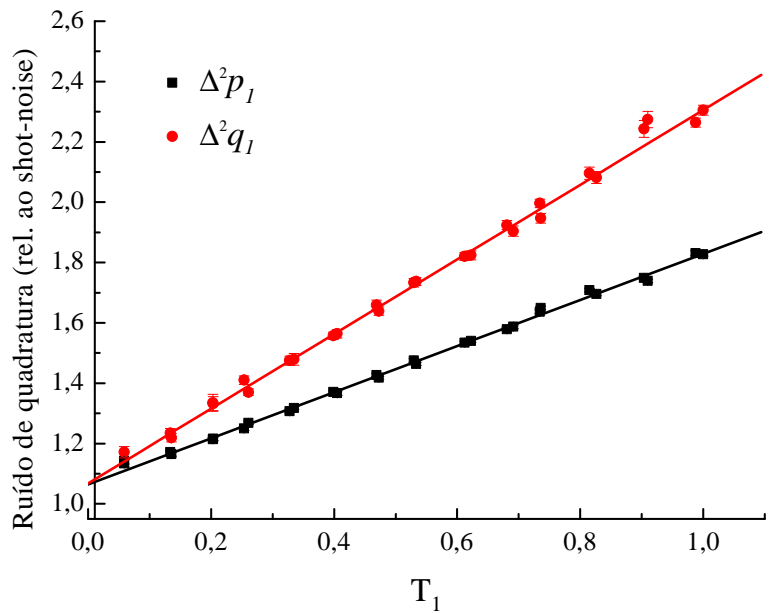

(a)

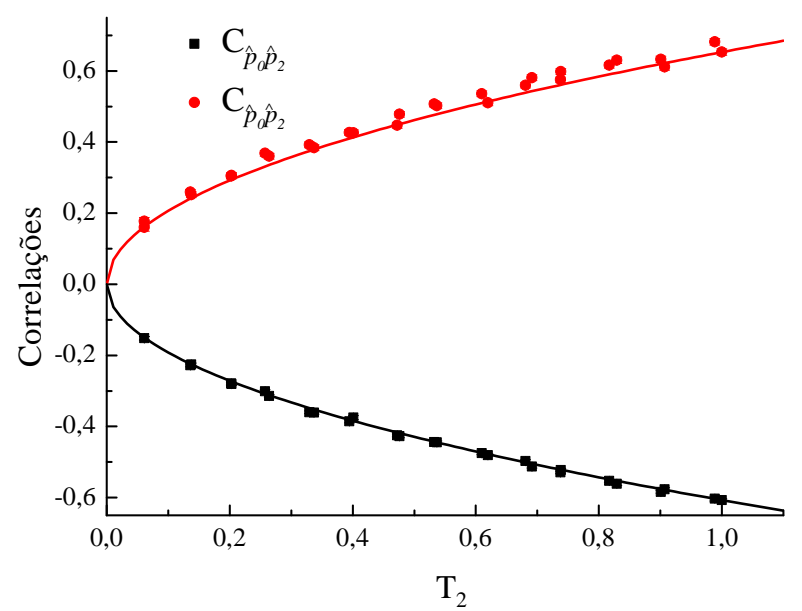

(c)

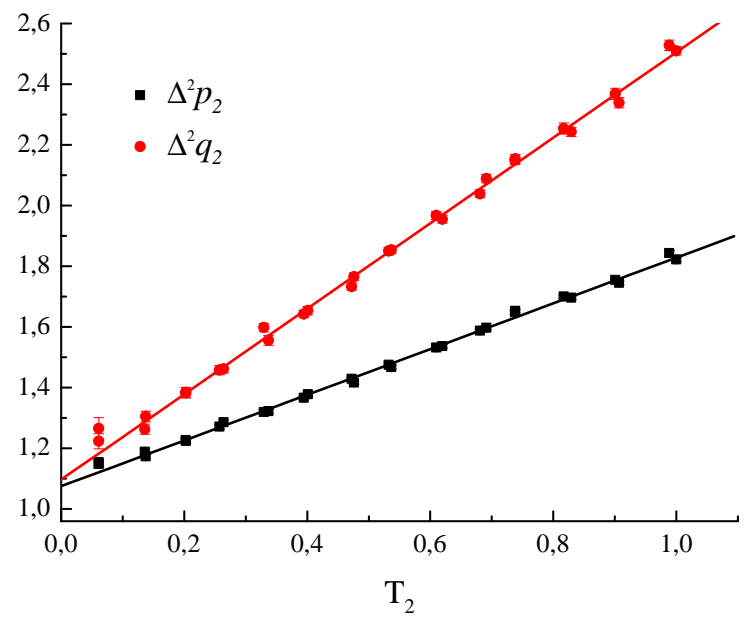

(b)

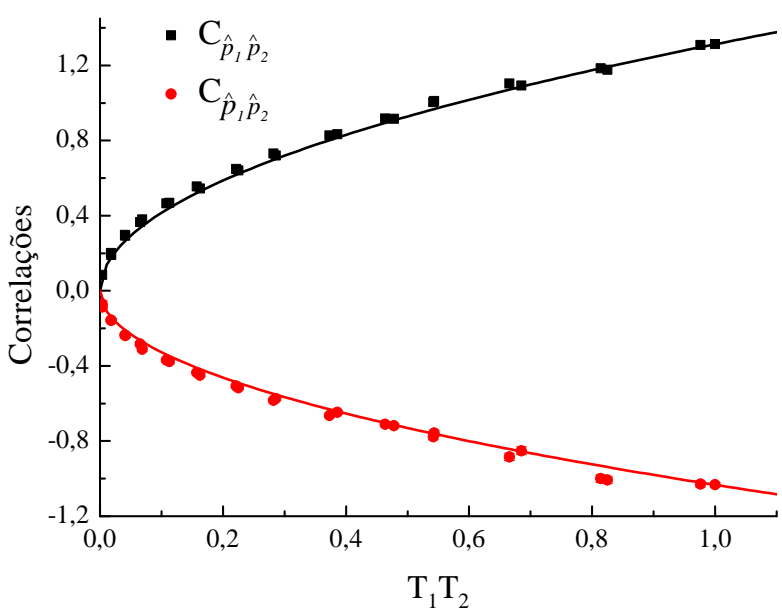

(d)

Figura 5.6: Teste da eletrônica de detecção. A potência do feixe de bombeio é mantida constante. Nas figuras (a) e (b) estão apresentados os ruídos de amplitude e fase dos feixes sinal e complementar, respectivamente. Verifica-se o comportamento linear em concordância com a equação (5.1.3), porém com valores diferentes da unidade para atenuação total. Correlações entre quadraturas são ilustradas em (c) para complementar e bombeio refletido e (d) para os feixes gêmeos, ambas em excelente acordo com a teoria.

essas quadraturas para diferentes valores de transmissão dos feixes gêmeos, mantendo sempre fixa a relação $T_{1}=T_{2}$. Os resultados obtidos estão ilustrados na figura 5.6.

Os gráficos 5.6(a) e 5.6(b) confirmam o comportamento linear esperado. No entanto, a intersecção da reta com o eixo vertical nos diz que, ao serem completamente atenuados, os ruídos não tendem à unidade. Este desvio torna necessária a análise da precisão na medida do shot-noise para identificar como a eletrônica do sistema influencia os resultados. Na figura 5.6(c) temos as correlações entre as amplitudes $\left(C_{\hat{p}_{0} \hat{p}_{2}}\right)$ e fases $\left(C_{\hat{q}_{0} \hat{q}_{2}}\right)$ dos feixes complementar e bombeio refletido em função de $T_{2}$, sempre mantendo $T_{0}=1$. Vemos um 
ótimo acordo entre os dados experimentais e a equação (5.1.4). O mesmo é observado para as correlações entre quadraturas $\left(C_{\hat{p}_{1} \hat{p}_{2}}\right.$ e $\left.C_{\hat{q}_{1} \hat{q}_{2}}\right)$ dos feixes gêmeos como função de $T_{1} T_{2}$, ilustradas no gráfico 5.6(d). Como o controle preciso da atenuação permite zerar completamente a transmissão dos feixes, podemos compreender facilmente o bom acordo entre os dados experimentais de correlação e a curva ajustada no limite de atenuação total, diferente do caso linear onde devemos tender a um valor que pode ser influenciado pela eletrônica envolvida.

Outra forma de avaliar o problema consiste em normalizar o ruído de subtração dos sinais pelo shot-noise, $\langle D C\rangle \times \beta$, e verificar se a média do conjunto de medidas desvia do valor unitário esperado. Os gráficos 5.7(a) e 5.7(b) mostram o comportamento como função da voltagem medida. Vê-se claramente que a eletrônica tem uma resposta dependente da intensidade do campo, o que coloca limitações na calibração do shot-noise por meio de uma constante comum a todas as medidas.

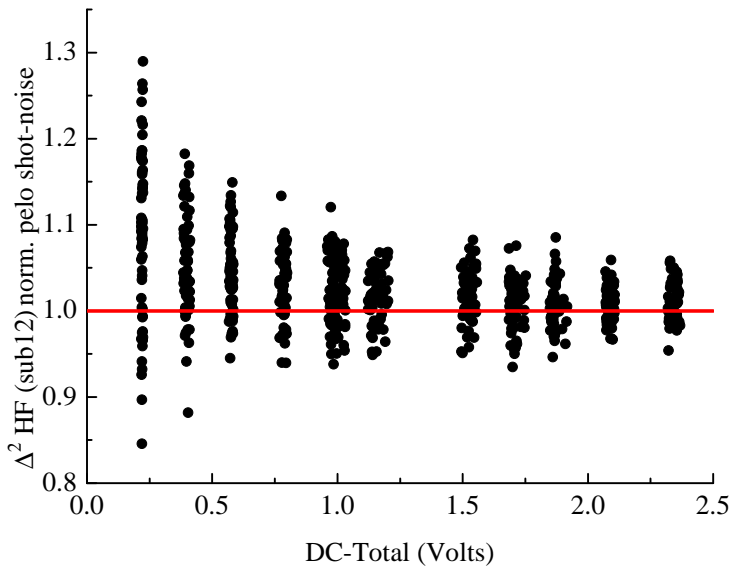

(a)

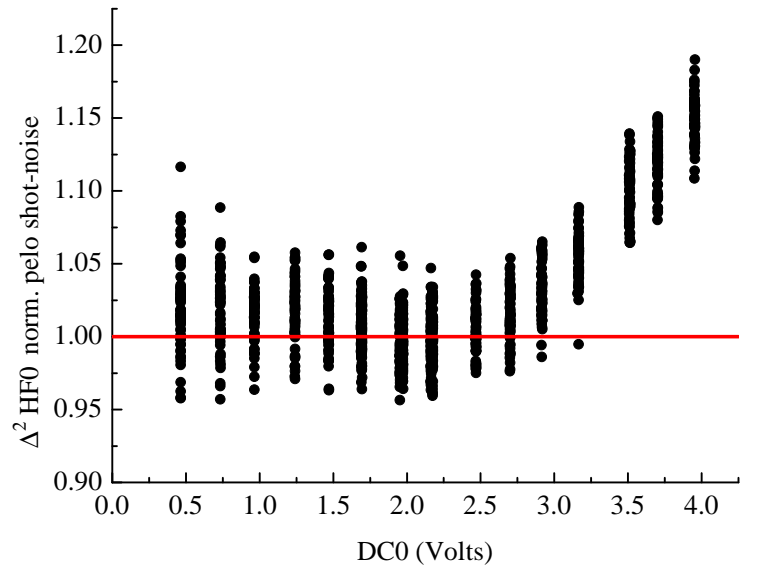

(b)

Figura 5.7: Análise da calibração do shot-noise por meio da normalização pelo fator $\langle D C\rangle \times \beta$. Em (a) temos o ruído da subtração dos sinais pelos pares correspondentes aos feixes gêmeos e em (b) o ruído do feixe de bombeio refletido.

A fim de diminuir essa imprecisão, a estratégia implementada consiste em modelar a resposta eletrônica introduzindo um ganho que varia linearmente com a intensidade do campo, $g(I)=g_{0}+g_{1} I$. A variância da fotocorrente medida é, portanto

$$
\Delta^{2} i=g(I) \Delta^{2} I+\Delta^{2} I_{e l e}
$$

Combinando a expressão $\Delta^{2} \hat{X}_{\varphi}=\langle I\rangle \Delta^{2} I$ obtida da equação (2.2.15) com (5.1.3), obtemos o ruído de intensidade do campo em função da transmissão $T$,

$$
\Delta^{2} I=T^{2}\left(\Delta^{2} I_{0}-I_{0}\right)+I_{0} T
$$




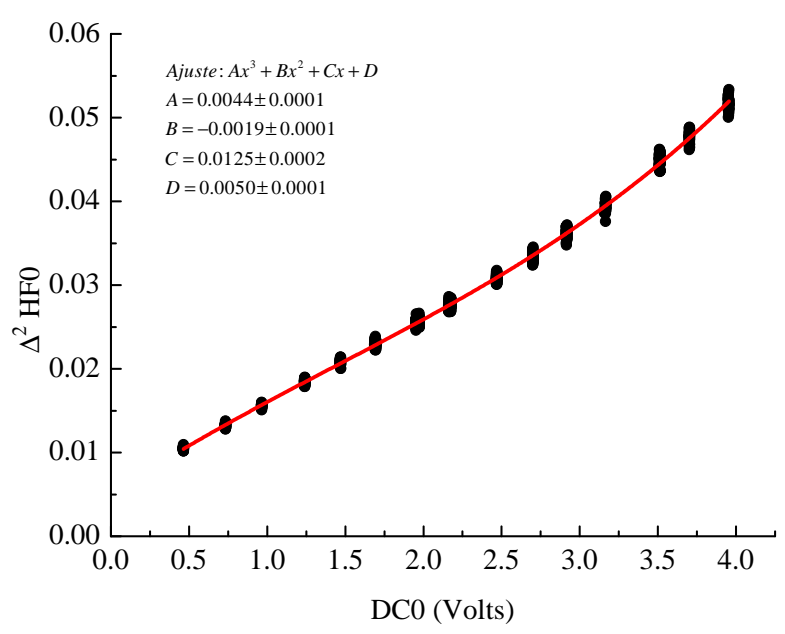

Figura 5.8: Ruído de intensidade do feixe bombeio refletido como função da intensidade do campo. O ruído eletrônico não foi descontado. Os coeficientes $C$ e $D$ nos fornecem o valor do shot-noise e o ruído eletrônico, respectivamente.

onde $I_{0}$ é a intensidade do campo não atenuado. Substituindo a equação (5.1.6) em (5.1.5), facilmente obtemos a expressão

$$
\begin{aligned}
\Delta^{2} i & =g_{1} \frac{\left(\Delta^{2} I_{0}-I_{0}\right)}{I_{0}^{2}} I_{0}^{3} T^{3}+\left[g_{0} \frac{\left(\Delta^{2} I_{0}-I_{0}\right)}{I_{0}^{2}}+g_{1}\right] I_{o}^{2} T^{2}+g_{0} I_{0} T+\Delta^{2} I_{\text {ele }} \\
& =A I^{3}+B I^{2}+C I+D,
\end{aligned}
$$

onde $I=I_{0} T$. Os coeficientes $A, B$ e $C$ tem suas expressões dadas por comparação direta entre as duas equações e seus valores obtidos a partir do melhor ajuste feito nos dados experimentais. Para exemplificar, fizemos o ajuste (figura 5.8) dos dados apresentados na figura 5.5(b) sem realizar a subtração do ruído eletrônico, dado pelo coeficiente $D$.

Verificamos uma correção de aproximadamente $20 \%$ no valor do ruído quântico. Esse tipo de calibração foi usada em parte dos resultados discutidos em breve.

\subsubsection{Calibração do shot-noise: Configuração 2}

Dada a imprecisão encontrada no método usual, buscamos uma forma mais confiável de calibração do shot-noise. Com o sistema de detecção 2, uma versão melhorada durante este trabalho de tese, calibramos o ruído quântico padrão de modo independente, para diferentes valores de intensidade, sendo o processo realizado para cada campo individualmente.

Uma vez enviado para o seu canal de detecção, o feixe de bombeio refletido (por exemplo) é dividido em dois outros feixes com igual intensidade (figura 5.4). Cada parte é enviada para seu respectivo fotodetector onde são medidos os sinais $H F 0 A$ e $H F 0 B$. Após realizar a calibração do ganho $g$ para os fotodetectores desse par, as amplitudes dos ruídos são calculadas. De acordo com as equações (2.6.18) e (2.6.19), o ruído da subtração 
das intensidades é proporcional ao valor médio da intensidade do campo; e o ruído da soma, como era de se esperar, igual ao ruído total. A razão $\Delta^{2} I_{+} / \Delta^{2} I_{-}$nos dá os ruídos de amplitude e fase do campo já normalizados pelo shot-noise, conforme visto na equação (2.6.21). As cavidades óticas vazias permitem acessar os ruídos da quadratura fase.

A seguir, mostramos a curva do ruído do feixe de bombeio normalizado pelo shot-noise. Trata-se da mesma análise feita na figura 5.7(b), porém com os dados obtidos com o novo esquema de medida.

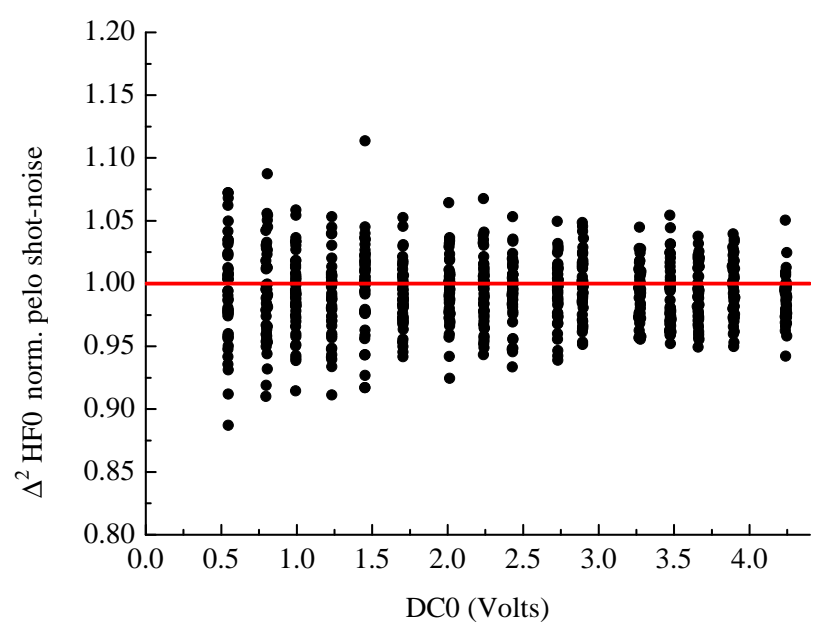

Figura 5.9: Ruído do feixe de bombeio normalizado pelo shot-noise. Ao passar pela cavidade de filtro, o laser passa a ser coerente o que justifica a dispersão no entorno de 1.0 .

A dispersão dos pontos permanece em torno de 1.0 para toda a faixa de potência, atestando que o feixe de bombeio é coerente. Como a calibração do ruído quântico padrão passa a ser feita ponto a ponto, esse novo esquema de medida oferece mais segurança no que diz respeito à sua calibração. Para cada aquisição, são calculados o ruído dos campos e sua normalização pelo shot-noise, procedimento feito com o OPO travado na ressonância, sem a necessidade de usar o feixe de prova em $1064 \mathrm{~nm}$ do Diabolo, pois são usados diretamente os campos de interesse.

A obtenção de parte dos resultados apresentados nesta tese foi possível somente por meio do sistema de detecção 2, conforme comentado nas seções seguintes.

\subsection{Medidas de emaranhamento tripartite}

A verificação experimental do emaranhamento tripartite se dá através da violação do critério de positividade sob transposição parcial (PPT), aplicado sobre a matriz de covariâncias do sistema, conforme vimos na seção 3.7.3.

A reconstrução da matriz de covariâncias ocorre através de medidas conjuntas das quadraturas dos campos. Variando a dessintonia de cada cavidade, acessamos em instan- 
tes distintos as quadraturas amplitude e fase de cada campo. Com a variação síncrona das dessintonias, temos acesso às correlações entre as amplitudes (fases) de campos distintos. Por fim, mantendo em ressonância uma cavidade por vez, conseguimos medir correlações entre quadraturas diferentes (i.e. amplitude de um campo e fase do outro). As larguras de banda temporais dos sinais de transmissão (e consequentemente reflexão) são ajustadas para valores tão próximos quanto possível, de modo a garantir que, estando em sincronia, as mesmas quadraturas dos três campos sejam adquiridas no mesmo instante.

A figura 5.10 ilustra os procedimentos adotados. Na primeira coluna, ilustramos a situação em que ocorre a variação síncrona das dessintonias das três cavidades, algo facilmente percebido observando que os mínimos das lorentzianas correspondentes aos sinais DC coincidem em $\Delta=0$, mostrando que as cavidades entram em ressonância no mesmo instante. Na figura central estão as curvas de ruído, ilustrando a alternância entre as quadraturas amplitude e fase. Em $|\Delta| \approx 0,5$ mede-se ruído de fase dos campos; em $|\Delta|=0$ e $|\Delta|>3$ observamos medidas correspondentes à quadratura amplitude. Por último, ilustramos as curvas de correlação entre os sinais de campos distintos, obtendo em $|\Delta| \approx 0,5$ as correlações de fase $\left(C_{\hat{q}_{i} \hat{q}_{j}}, i, j=0,1\right.$ e 2 com $\left.i \neq j\right)$ e em $|\Delta|>3$ correlações de amplitude $\left(C_{\hat{p}_{i} \hat{p}_{j}}, i, j=0,1\right.$ e 2 com $\left.i \neq j\right)$.

Na segunda coluna da figura 5.10, mostramos o resultado obtido ao variar a dessintonia de uma cavidade (neste caso, a do feixe sinal) mantendo completamente fora de ressonância as outras duas. Com este procedimento verificamos a alternância de quadraturas somente para o campo cuja cavidade tem sua dessintonia alterada, enquanto as outras fornecem somente a quadratura amplitude por estarem fora de ressonância. Dessa forma, ao repetir o procedimento para as três cavidades, medimos os valores das correlações cruzadas $\left(C_{\hat{q}_{i} \hat{p}_{j}}, i, j=0,1\right.$ e 2 com $\left.i \neq j\right)$ para $|\Delta| \approx 0,5$, completando todas as entradas da matriz de covariâncias.

Os ajustes não-lineares das funções (2.7.40) e (2.7.43) das curvas medidas fornecem os termos da matriz de covariâncias, deixados como parâmetros livres, e suas respectivas barras de erro. De um modo geral, primeiro são ajustadas as lorentzianas dos sinais de reflexão da cavidade, obtendo o ponto de ressonância correspondente ao mínimo do sinal DC, a largura de banda temporal da cavidade e o valor da reflexão mínima $\left(R_{\min }\right)$. Estes parâmetros permanecem fixos nos ajustes das curvas de ruído e correlações. Como pode ser visto na figura 5.10, há um excelente acordo entre teoria e experimento. Uma vez obtidas as grandezas de interesse, construímos a matriz de covariâncias e aplicamos o critério PPT, segundo explicado na seção 3.7.3.

Para demonstrar o emaranhamento tripartite medimos a matriz de covariâncias para diferentes valores de potência de bombeio relativa ao limiar de oscilação, $\sigma=P / P_{\text {lim }}$. Para cada $\sigma$, aplicamos o critério PPT, calculando o menor autovalor simplético da matriz gerada pela transposição parcial de um dos campos. Os resultados obtidos estão apresentados na figura 5.11. Os pontos em azul e vermelho $\left(\nu_{1}\right.$ e $\left.\nu_{2}\right)$ são referentes à 

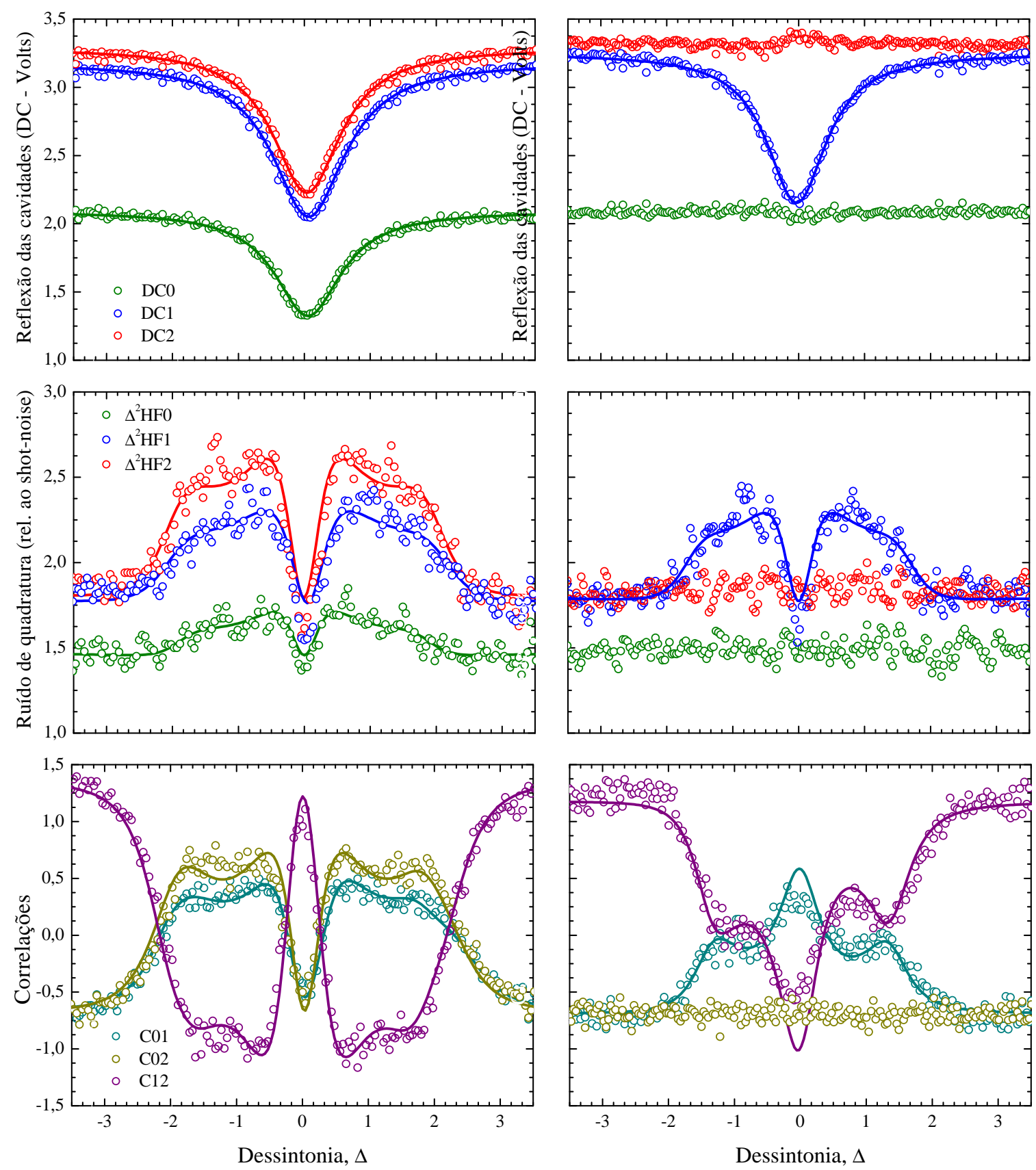

Figura 5.10: Construção experimental da matriz de covariâncias. Na coluna da esquerda, estão ilustrados os resultados obtidos quando as dessintonias das três cavidades variam de modo síncrono $\left(\Delta_{i}=\Delta, i=0,1\right.$ e 2$)$, o que pode ser visto a partir do sinal DC na figura superior. Nesta condição, obtemos os ruídos de amplitude e fase de cada campo e as correlações de quadraturas iguais $\left(C_{\hat{p}_{i} \hat{p}_{j}}\right.$ e $C_{\hat{q}_{i} \hat{q}_{j}}, i, j=0,1$ e 2 com $\left.i \neq j\right)$. Na coluna da direita temos somente uma cavidade com variação de dessintonia (gráfico azul), mantendo as demais (vermelho e verde) completamente fora de ressonância, situação em que é medida somente a quadratura amplitude. Essa configuração permite obter correlações de quadraturas distintas $\left(C_{\hat{q}_{i} \hat{p}_{j}}, i, j=0,1\right.$ e 2 com $\left.i \neq j\right)$. O procedimento deve ser realizado para as três cavidades. 

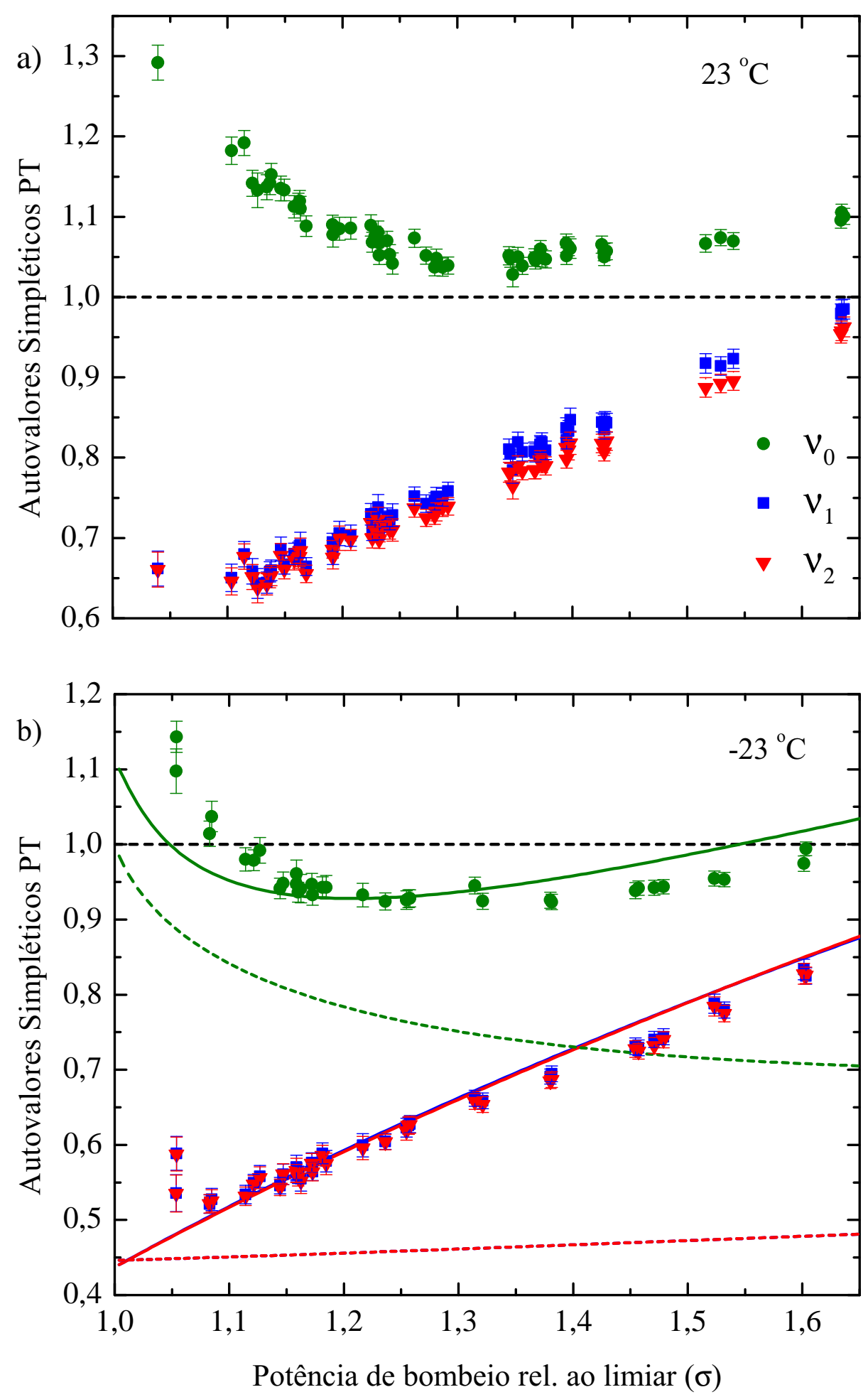

Figura 5.11: Autovalores simpléticos extraídos das matrizes de covariâncias medidas para diferentes valores de potência de bombeio, $\sigma=P / P_{\text {lim }}$. O subsistema transposto é: feixe bombeio (círculos verdes), sinal (quadrados azuis) e complementar (triângulos vermelhos). Em (a) e (b) apresentamos medidas realizadas em $T=23{ }^{\circ} \mathrm{C}$ e $T=-23{ }^{\circ} \mathrm{C}$, respectivamente, sendo o emaranhamento tripartite verificado somente em (b). As linhas tracejadas de cor verde e vermelha no gráfico de baixo são ajustes feitos sem incluir o excesso de ruído que surge por causa da interação com fônons do cristal não-linear. 
transposição parcial dos feixes sinal e complementar, respectivamente, e os de cor verde $\left(\nu_{0}\right)$ referem-se à transposição do feixe de bombeio refletido.

As medidas foram feitas em duas condições diferentes de operação: uma fixando a temperatura do cristal em $T=23{ }^{\circ} \mathrm{C}$ e outra em $T=-23{ }^{\circ} \mathrm{C}$. Percebemos de forma clara a influência da temperatura nos resultados. O gráfico 5.11(a) mostra que não existe emaranhamento entre os feixes gêmeos e o feixe de bombeio refletido, pois $\nu_{0}>1$ para qualquer valor de $\sigma$. Quando $T=-23{ }^{\circ} \mathrm{C}$, observamos $\nu_{0}<1$, para uma ampla faixa de valores de potência de bombeio, o que demonstra de forma inequívoca o emaranhamento tripartite.

Nestes gráficos também estão incluídos os ajustes teóricos provenientes do formalismo matricial apresentado na seção 4.5. Para implementá-los basta utilizar a matriz de covariâncias $V_{\mathrm{OPO}}$ definida na equação (4.5.10), adicionando as matrizes cujas contribuições desejamos estudar. As linhas tracejadas do gráfico 5.11 foram obtidas por meio do ajuste da equação (4.5.11), ou seja, sem levar em conta o ruído de fônons. Para que este efeito seja incluído, é preciso adicionar a matriz (4.5.15) à equação (4.5.11) e ajustar a teoria aos dados experimentais, de onde serão obtidas as curvas contínuas, utilizando os parâmetros do experimento no cálculo.

Este resultado mostra a primeira medida de emaranhamento entre mais de dois subsistemas produzidos por uma única fonte, tendo como característica especial a diferença entre os comprimentos de onda dos campos envolvidos. Para esta temperatura, foram medidos os seguintes comprimentos de onda: $\lambda_{0}=532.251 \mathrm{~nm}, \lambda_{1}=1062.102 \mathrm{~nm}$, e $\lambda_{2}=1066.915 \mathrm{~nm}$.

As barras de erro dos autovalores simpléticos são calculadas via simulações via método de Monte Carlo. Do ajuste não-linear das curvas 5.10, obtemos os valores das entradas da matriz de covariâncias e os erros associados a cada uma. Para obter o erro dos autovalores simpléticos, sorteamos uma nova matriz de covariâncias a partir da matriz medida e de suas incertezas. Cada elemento da nova matriz é calculado como a soma do elemento da matriz medida com uma distribuição gaussiana aleatória de média nula multiplicada pelo erro da matriz medida. São realizadas 10 mil simulações, cada uma fornecendo um autovalor simplético. Feito isso, um histograma é construído e dele é extraído o erro dos $\nu_{i}(i=0,1,2)$. Os detalhes deste procedimento são dados na referência [Cassemiro 2008].

A distinção encontrada entre os resultados obtidos em diferentes temperaturas se deve à existência de um ruído clássico espúrio inserido pela interação dos campos com fônons da rede cristalina [César 2008b]. Este fenômeno mascarou por muito tempo o emaranhamento existente entre os três feixes produzidos pelo OPO [Cassemiro 2007]. Podemos constatar seu efeito comparando os valores dos ruídos de quadratura dos feixes quando submetemos o cristal não-linear a diferentes temperaturas. Na figura 5.12 apresentamos comportamento para as duas situações apresentadas, $T=23{ }^{\circ} \mathrm{C}$ e $T=-23{ }^{\circ} \mathrm{C}$. Comparando os gráficos 5.12(a) e 5.12(b) não observamos uma mudança significativa nos valores 
dos ruídos de amplitude dos campos, mostrando que estes não são alterados pela variação de temperatura. Porém, ao comparar 5.12(c) e 5.12(d) notamos uma clara redução no ruído de fase dos feixes ao esfriar o cristal. As linhas tracejadas não possuem significado físico e foram incluídas somente para facilitar a visualização da diferença de valores nas duas situações.

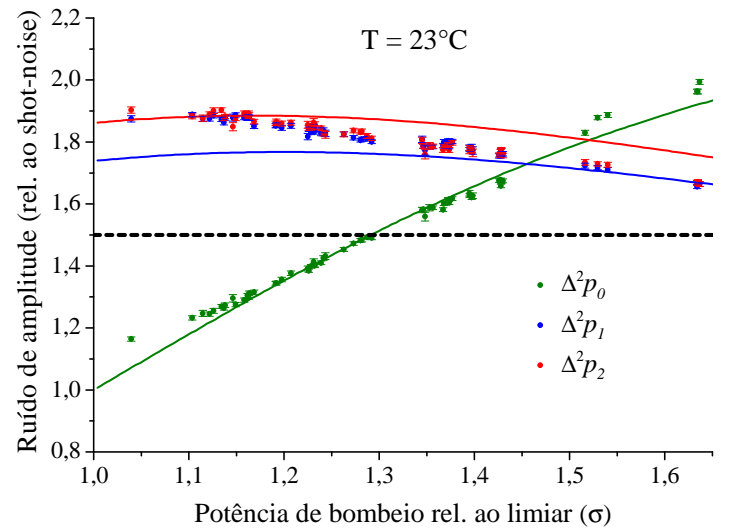

(a)

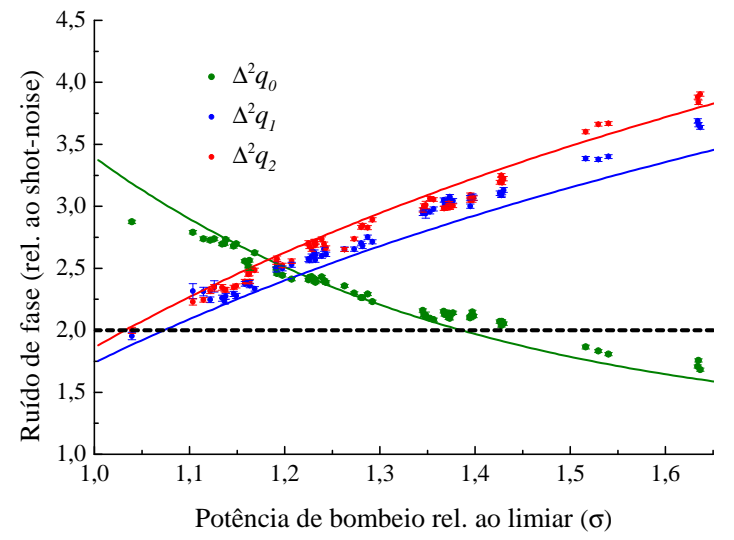

(c)

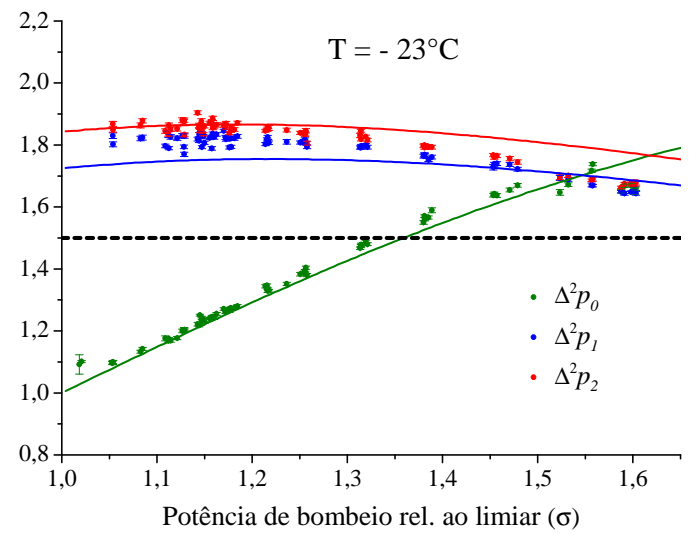

(b)

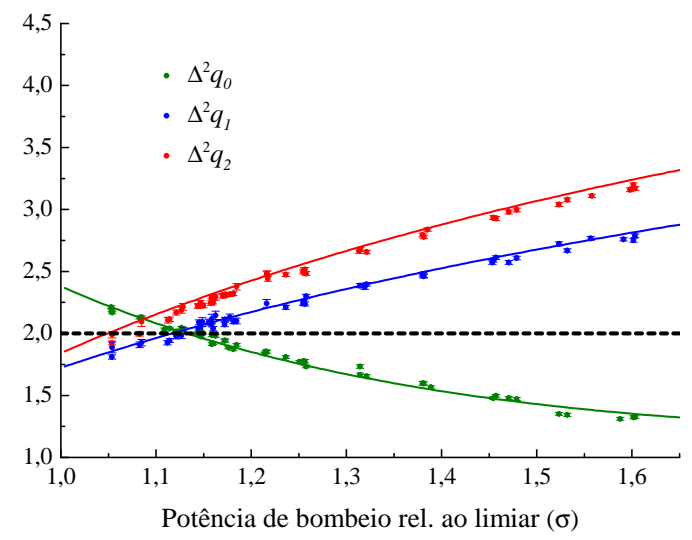

(d)

Figura 5.12: Medidas dos ruídos de quadraturas dos feixes bombeio refletido, sinal e complementar mantendo o cristal à temperatura ambiente (gráficos 5.12 (a) e 5.12(c)) e à -23 ${ }^{\circ} \mathrm{C}$ (gráficos 5.12(b) e 5.12(d)). É observado uma diminuição do ruído da quadratura fase, sem alteração do nível de ruído da quadratura amplitude. Esta é a principal justificativa para a observação do emaranhamento tripartite somente em baixas temperaturas. As linhas contínuas se referem ao melhor ajuste teórico dos elementos da matriz (4.5.11) incluindo o efeito do excesso de ruído presente na matriz (??), considerando os parâmetros medidos no experimento. As linhas tracejadas, desprovidas de significado físico, foram incluídas somente para efeito de comparação.

O modelo teórico que explica esse comportamento leva em consideração pequenas flutuações na permissividade do cristal, mudando o índice de refração do meio devido a 
flutuações locais na densidade, associadas às vibrações da rede. Também é explicado pelo mesmo modelo o fato de não haver variação no ruído de amplitude do campo, sendo afetadas principalmente as flutuações de fase [Coelho 2009a]. No gráfico 5.11(b) mostramos o acordo entre teoria e experimento. As curvas tracejadas são obtidas com a teoria usual do OPO [Villar 2007b], sem levar em consideração o ruído de fônons. As linhas sólidas levam em consideração este efeito e se ajustam muito bem aos dados experimentais.

Uma vez medido o emaranhamento somos tentados a criar uma visão ainda mais otimista: utilizá-lo como recurso para implementar tarefas no contexto da informação quântica. Sabemos que para armazenar informação, meios materiais tais como átomos e íons são fortes candidatos e, para transmissão, a luz desempenha papel fundamental, dada sua pouca interação com o ambiente e alta velocidade. Porém, é de grande utilidade entender mais sobre a dinâmica do emaranhamento e visualizar seus limites de aplicação. O objetivo das próximas sessões é justo tentar identificar quão frágil é o emaranhamento produzido pelo OPO e quais regiões de parâmetros viabilizam sua aplicação.

\subsection{Morte súbita de emaranhamento}

O emaranhamento desempenha papel fundamental na execução de tarefas em computação e informação quântica, mas é uma propriedade facilmente degradada quando o sistema interage com o ambiente, originando perda de correlações entre os constituintes. Na prática, qualquer sistema físico está sujeito a descoerência e em algumas situações a dinâmica do emaranhamento apresenta uma perda repentina de correlações, conhecida como morte súbita de emaranhamento. Os primeiros estudos teóricos deste fenômeno foram feitos no âmbito de variáveis discretas, mostrando que, embora a coerência das partes sofra um decaimento exponencial, o emaranhamento se perde para um tempo finito [Yu 2006]. Não demorou muito para que a morte súbita fosse demonstrada experimentalmente em sistemas de variáveis discretas [Almeida 2007] e prevista em sistemas de variáveis contínuas [Paz 2008].

Em nosso sistema investigamos o comportamento do emaranhamento entre os feixes sinal, complementar e bombeio refletido quando submetidos a perdas impostas por atenuadores controláveis.

\subsubsection{Caso tripartite}

Após a obtenção do emaranhamento tripartite, começamos a investigar a dinâmica do emaranhamento entre os feixes quando os mesmos são submetidos a perdas, seguindo o mesmo procedimento de atenuação descrito na seção 5.1.1 .

Em um sistema envolvendo dois campos comprimidos, os ruídos de amplitude e fase se aproximam linearmente do ruído quântico padrão e as correlações tendem a zero quando a 
transmissão vai a zero $(T \rightarrow 0)$, conforme mostram as equações (5.1.3) e (5.1.4). Esperase, portanto, separabilidade dos feixes somente quando os campos são completamente perdidos.

Em nosso experimento, atenuamos igualmente os feixes sinal e complementar, de modo que $T_{1}=T_{2}=T$, medindo a matriz de covariâncias correspondente a cada valor de $T$. Feito isso, extraímos os autovalores simpléticos de cada matriz, conforme apresentado na seção 3.7.3, e observamos o comportamento dos mesmos em função de $T$.

Verificamos, diferentemente do esperado a priori, o desemaranhamento entre o feixe de bombeio refletido e os campos atenuados para perdas finitas $(0<T<1)$, obtendo, portanto, uma demonstração experimental de um fenômeno análogo à morte súbita de emaranhamento em variáveis contínuas. Este efeito é observado para potências de bombeio relativamente baixas $(\sigma=1.14)$, conforme ilustrado na figura 5.13(a), e se torna menos evidente à medida que a aumentamos. Vemos na figura 5.13(c) que o fenômeno ainda existe para $\sigma=1.17$, no entanto com o indicativo de que o emaranhamento é mais resistente às perdas neste regime. Ao aumentar consideravelmente a potência de bombeio, verificamos que o desemaranhamento deixa de ser observado, figura 5.13(d). Esta característica indica que os estados emaranhados produzidos pelo OPO, para diferentes $\sigma$ 's, não possuem a mesma natureza, muito embora as flutuações do campo continuem sendo de origem gaussiana para todos os valores de potência de bombeio, de acordo com o que será apresentado na seção 5.4. As linhas sólidas são obtidas por meio da equação (4.5.23), que nada mais é do que uma atenuação numérica realizada nos feixes gêmeos a partir da matriz de covariâncias medida sem qualquer atenuação.

Os estados dos três campos que se mantêm emaranhados para qualquer valor de atenuação são denominados robustos. Trata-se de uma classe muito importante de estados que assegura a existência de emaranhamento mesmo em canais nos quais os campos estejam sujeitos a perdas por absorção ou espalhamento.

Outra informação importante que pode ser extraída deste estudo diz respeito à estrutura do emaranhamento. Podemos, por exemplo, efetuar um traço parcial em um dos feixes e verificar se o emaranhamento entre os outros dois campos ainda persiste. Isto pode ser feito para todos os valores de atenuação. Vamos considerar um estado obtido por meio de simulação numérica, ilustrado na figura 5.14.

Os gráficos apresentados em 5.14 foram obtidos a partir de uma matriz de covariâncias experimental. Utilizando a equação (4.5.23), foram adicionadas perdas na matriz medida, de onde obtemos a linha continua. Para determinados valores de atenuação, foi adicionado um ruído estocástico por simulação de Monte Carlo na matriz atenuada, gerando uma nova matriz de onde extraímos os autovalores simpléticos dados pelos pontos nos gráficos. Isso foi feito com o intuito de simular um processo de atenuação experimental. Para cada simulação o menor autovalor simplético correspondente é calculado.

O gráfico 5.14(a) foi obtido pela transposição parcial de um campo. Trata-se de uma 

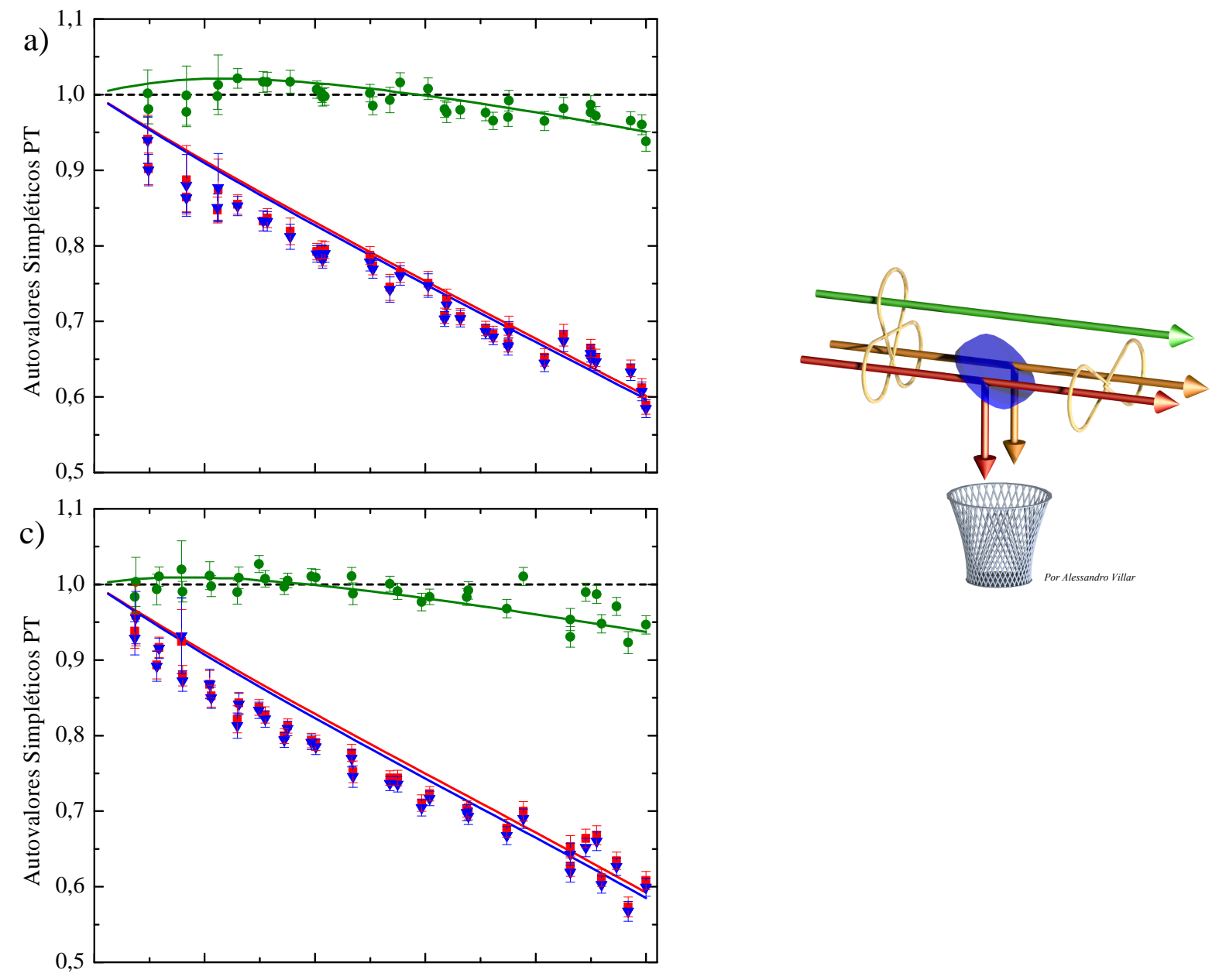

(b)
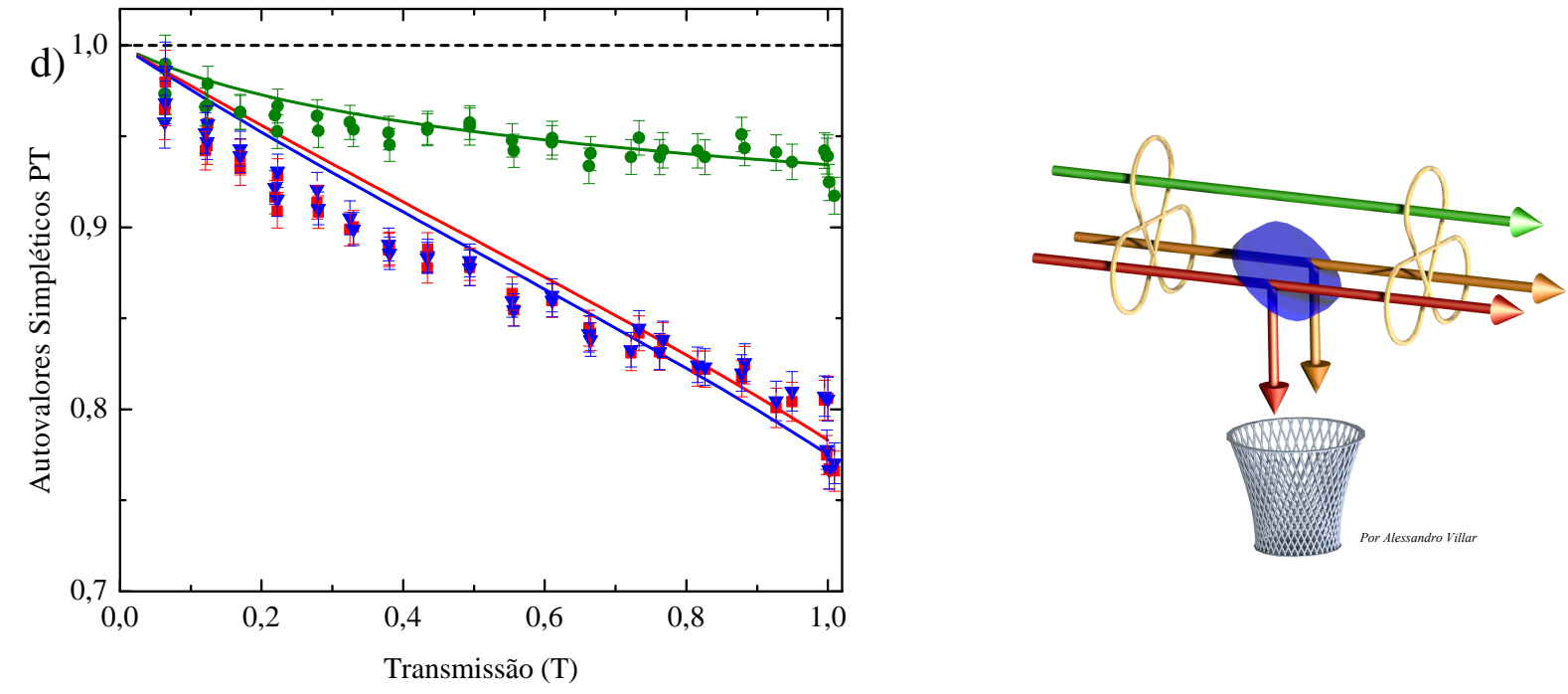

(e)

Figura 5.13: Autovalores simpléticos em função das perdas para valores diferentes de potência de bombeio, (a) $\sigma=1,14$, (c) $\sigma=1,17$ e (d) $\sigma=1,40$. As curvas sólidas correspondem a ajustes realizados numericamente a partir das matrizes de covariâncias medidas sem atenuação. As figuras (b) e (e) nos dão uma visão pictórica do efeito das perdas sobre o estado formado pelos três campos. 
matriz de covariâncias medida para uma região de parâmetros que apresenta emaranhamento tripartite robusto. Na figura 5.14(b), apresentamos os autovalores simpléticos das matrizes reduzidas, obtidas pelo traço parcial de um dos campos. Por exemplo, o autovalor simplético $\nu_{01}$ indica que estamos lidando com o sistema bipartite formado pelo feixe de bombeio refletido e o feixe sinal, pois foi realizado o traço parcial sobre o feixe complementar. O mesmo raciocínio deve ser empregado para os demais autovalores.

A informação relevante que pode ser extraída do gráfico 5.14(b) é que, para transmissões menores que 0.6, nenhuma bipartição apresenta emaranhamento, mesmo com a presença de emaranhamento tripartite. Se este fato for comprovado experimentalmente, algo que deve ser alcançado de forma empírica, investigando a região de parâmetros favorável, teremos a produção de um estado análogo ao GHZ em variáveis contínuas, já produzido e controlado experimentalmente para qubits [Roos 2004, Dür 2000]. Outra classe de estados que, em princípio, pode ser obtida é análoga aos estados $W$, que apresenta emaranhamento em todas as configurações possíveis: sistema global tripartite e bipartições. Dessa forma, a inserção de perdas controladas no estado tripartite possibilitaria uma transição entre o análogo $W$ e o análogo GHZ em variáveis contínuas.

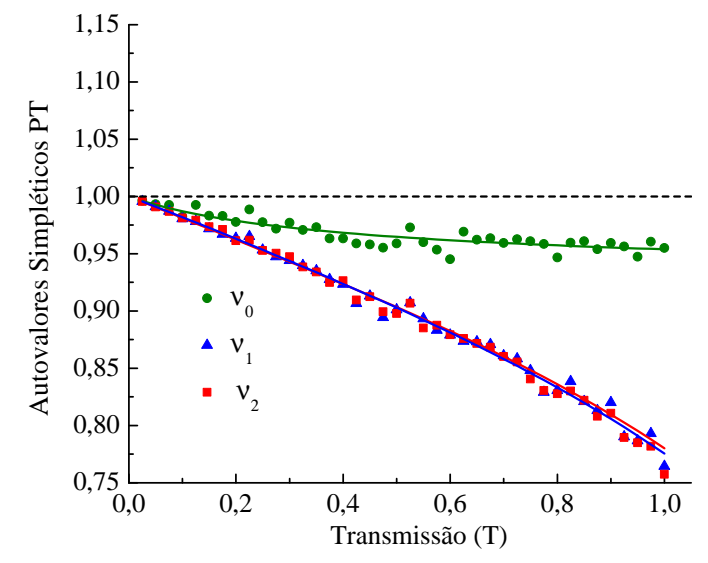

(a)

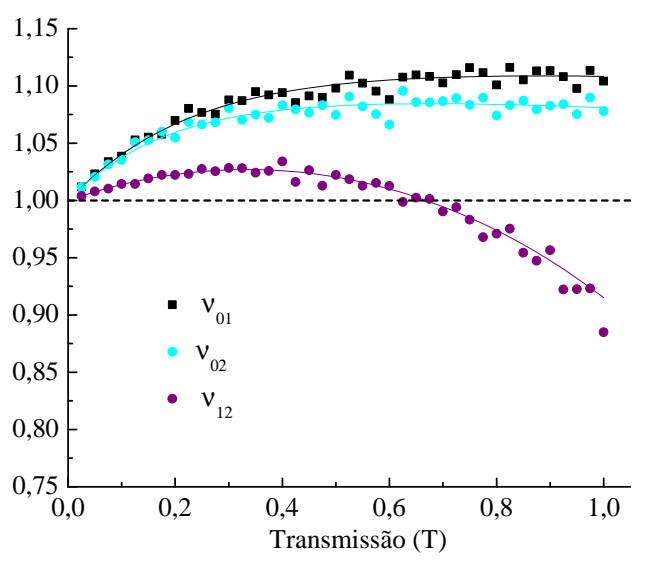

(b)

Figura 5.14: Aplicação do critério PPT para o diferentes partições. Em 5.14(a) consideramos o sistemas global tripartite, transpondo somente um campo. Em 5.14(b) analisamos o sistema bipartite obtido pelo traço parcial sobre um dos campos. Autovalores simpléticos menores que 1 indicam emaranhamento, e maiores que 1 separabilidade.

Verificamos aqui, um fenômeno análogo à morte súbita de emaranhamento no âmbito das variáveis contínuas [Coelho 2009b] e investigamos como a estrutura do emaranhamento pode ser investigada experimentalmente. A seguir, mostraremos que o desemaranhamento para perdas finitas não é fruto somente da complexidade do sistema tripartite, pois também é observado no sistema emaranhado mais simples, o bipartite, formado pelos feixes sinal e complementar. 


\subsubsection{Morte súbita bipartite}

Identificamos no sistema tripartite uma dinâmica de emaranhamento dependente dos parâmetros envolvidos no experimento. Para potências de bombeio próximas ao limiar de oscilação o emaranhamento se mostra frágil frente às perdas, desaparecendo para valores de atenuação próximos de 50\%. Potências mais altas dão origem a um emaranhamento robusto, indo a zero somente para perda total. Podemos pensar que essa característica se deve à complexidade do sistema tripartite, visto que a atenuação dos feixes gêmeos gera perda de correlações com o feixe de bombeio refletido. No entanto, analisando o subespaço formado pelos feixes sinal e complementar, mostramos que este efeito também está presente no sistema bipartite.

Após medir matrizes de covariâncias para diversas potências de bombeio, simulamos numericamente o efeito da atenuação sobre os campos, identificando as regiões de parâmetros onde o emaranhamento é frágil quando submetido a perdas. Esta análise mostrou que a transição de estados emaranhados para separáveis é bastante sutil, pois a violação do critério PPT seria obtida para autovalores simpléticos muito próximos de um. Foi esta análise que motivou a implantar o sistema de detecção 2, detalhado anteriormente.

Os estados produzidos pelo OPO podem ser interpretados como estados do tipo EPR no que diz respeito à subtração das amplitudes $\left(\hat{p}_{-}=\hat{p}_{1}-\hat{p}_{2}\right)$ e soma das fases $\left(\hat{q}_{+}=\right.$ $\hat{q}_{1}+\hat{q}_{2}$ ) dos campos. Foi demonstrado por Villar et al. [Villar 2005] que estados produzidos com potência de bombeio muito próxima ao limiar de oscilação violam a desigualdade de Duan [Duan 2000]

$$
\Delta^{2} \hat{p}_{-}+\Delta^{2} \hat{q}_{+} \geq 2
$$

onde as variâncias são normalizadas pelo ruído quântico padrão, igual à um. Este é um critério de emaranhamento suficiente, pois podem existir estados emaranhados que não o violem. No entanto, é fácil ver que estados comprimidos de dois modos que não satisfazem a desigualdade de Duan apresentam robustez quando submetidos à atenuação. Para o caso simétrico (atenuações iguais nos dois campos), mostra-se de modo simples que, em função da atenuação, a desigualdade (5.3.1) é reescrita como

$$
\Delta^{2} \hat{p}_{-, T}+\Delta^{2} \hat{q}_{+, T}=T\left(\Delta^{2} \hat{p}_{-}+\Delta^{2} \hat{q}_{+}\right)+2(1-T),
$$

de onde se vê que, uma vez violada, a desigualdade de Duan permanece assim para todos os valores de transmissão. Quando a transmissão se torna nula teremos somente modos do vácuo sendo medidos, o que nos leva à igualdade da equação (5.3.1).

Contudo, por ser somente um critério suficiente, podem existir estados emaranhados que não sejam robustos e apresentem perda de emaranhamento para valores finitos de atenuação. Por conta disso usamos o critério PPT, necessário e suficiente para estados gaussianos. O processo experimental é o mesmo descrito para o caso tripartite, porém a ideia se baseia em um caso prático simples. Supondo haver troca de informação entre duas 

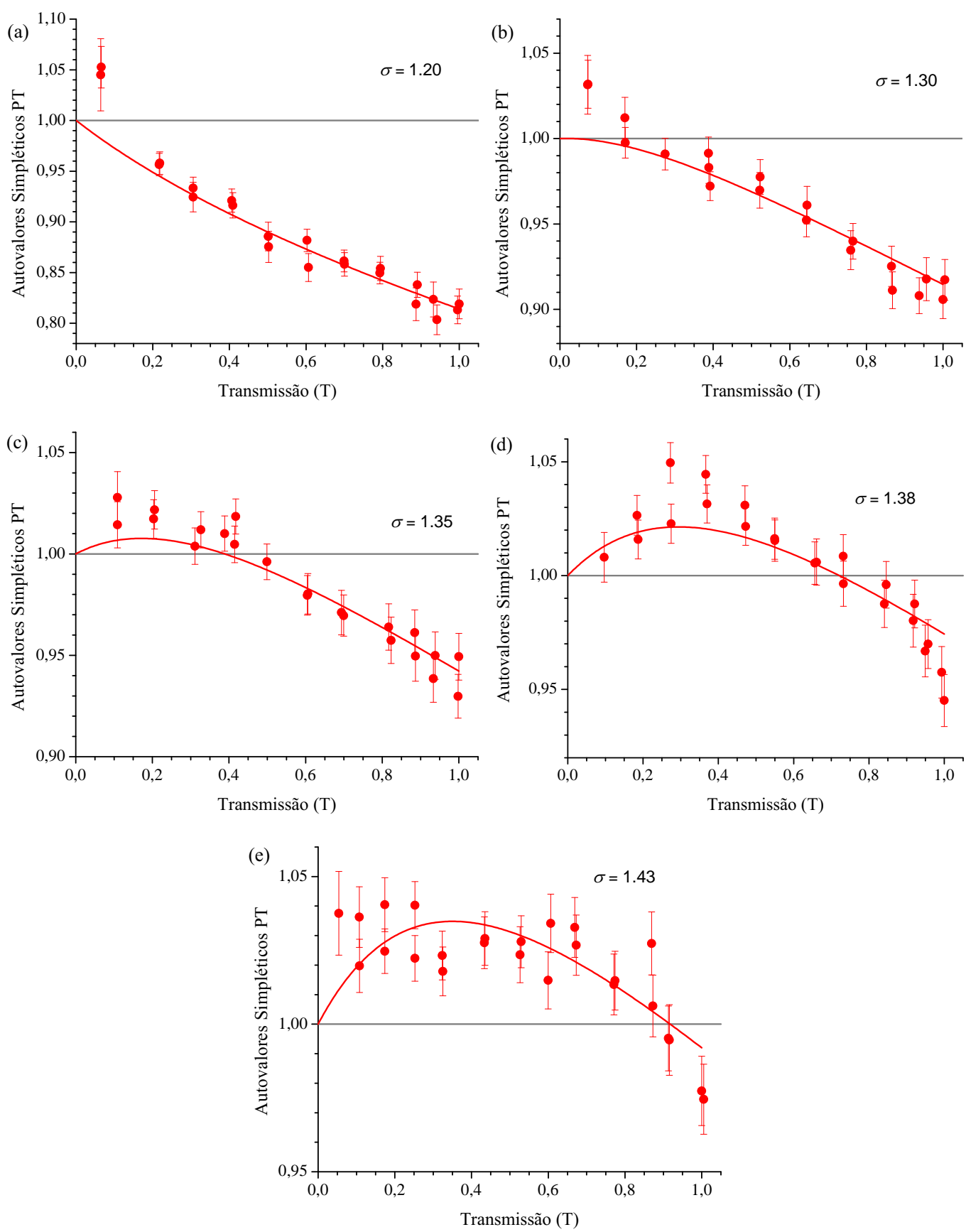

Figura 5.15: Autovalores simpléticos em função das perdas de um feixe para valores diferentes de potência de bombeio relativa ao limiar de oscilação $(\sigma)$. Os valores de $\sigma$ estão indicados em cada gráfico. As curvas sólidas correspondem a ajustes da equação 4.5.23, realizados numericamente a partir da matriz de covariâncias medida sem atenuação. Percebe-se claramente a transição de um estado robusto para um estado frágil à medida que aumentamos a potência de bombeio e consequentemente a interação com os fônons da rede cristalina.

partes, imaginamos a situação em que uma delas está com a fonte de estados emaranhados 
e envia um dos campos para a outra. Neste caso, somente o feixe enviado estará suscetível às perdas. Os resultados foram obtidos atenuando somente um dos campos.

Caracterizamos o efeito das perdas no emaranhamento presente nos feixes gêmeos para diferentes valores de potência de bombeio relativas ao limiar de oscilação. Os resultados obtidos estão ilustrados na figura 5.15. Para potências de bombeio muito próximas do limiar de oscilação, já é bem conhecida em nosso experimento a violação do critério de Duan et al. (equação 5.3.1), que deve permanecer violada para qualquer valor de atenuação. Neste caso o estado é do tipo robusto frente às perdas, conforme verificado na figura 5.15(a). À medida que aumentamos a potência do feixe de bombeio, inserimos mais ruído de fase nos feixes gêmeos o que gera degradação do emaranhamento e leva o estado a ser do tipo frágil.

A figura 5.15 mostra a degradação e a fragilidade do emaranhamento com o aumento da potência de bombeio $(\sigma)$. No caso ideal, onde não há ruído espúrio, o desemaranhamento não está presente, o que nos leva a crer que a presença de ruído de fônons tem um papel importante no processo. Este estudo permite delimitar fronteiras entre estados robustos, frágeis e separáveis produzidos pelo OPO que podem ser cruzadas através de parâmetros físicos controláveis, tais como temperatura do cristal e potência de bombeio. Outro estudante do grupo, Felippe A. S. Barbosa, esteve diretamente ligado a esse trabalho e mais detalhes sobre a classificação desses estados poderão ser encontrados nas referências [Barbosa 2010a, Barbosa 2010b], e em sua tese de doutorado.

Estes resultados mostram a existência de perda de emaranhamento para atenuações finitas, identificando regiões de parâmetros que podem ser interessantes para o uso do nosso sistema no contexto da informação quântica. Além disso, o trabalho também permitiu estudar as fronteiras existentes entre diferentes classes de emaranhamento que surgem no contexto de perdas finitas. Entender o caso mais simples pode ser útil no entendimento de casos mais complexos como o tripartite.

\subsection{Teste de gaussianidade}

Os resultados anteriores se baseiam na aplicação do critério PPT, necessário e suficiente para estados gaussianos, o que torna relevante a análise estatística das flutuações dos campos. A análise seguinte se baseia numa consequência direta do teorema central do limite para distribuições que possuem todos os momentos finitos: duas distribuições são idênticas se todos os seus momentos coincidem [Krauth 1998].

Assim sendo, comparando os momentos de ordem superior de uma distribuição gaussiana com momentos de ordem superior das quadraturas amplitude e fase dos feixes gerados pelo OPO, podemos verificar a gaussianidade dos estados produzidos. Para uma distri- 
buição gaussiana os momentos de ordem par devem obedecer uma escala bem definida,

$$
\gamma \equiv \frac{\left\langle X^{2 n}\right\rangle}{\vartheta^{2 n}}=(2 n-1) ! !
$$

onde $\vartheta$ representa o desvio padrão, de acordo com a discussão apresentada na seção 4.6. Além disso, deve-se checar se todos os momentos de ordem ímpar são nulos, conforme esperado para uma distribuição normal. Dessa forma, os dois primeiros momentos da distribuição contêm toda a informação sobre o estado, de modo especial através da matriz de covariâncias do sistema.

Apresentamos a seguir os resultados dos cálculos dos momentos de ordem superior realizados para as flutuações dos três campos gerados pelo OPO operando em diferentes potências de bombeio relativas ao limiar. A realização das medidas de flutuações de amplitude é tecnicamente mais simples quando comparada com medidas da quadratura fase, pois para a primeira basta deixarmos as cavidades de análise fora de ressonância, enquanto a segunda exige o travamento do comprimento das cavidades à meia altura do sinal de transmissão (ou reflexão), ponto onde as flutuações de fase são acessadas. Por conta disso, uma melhor estatística é obtida para os momentos de ordem superior normalizados pelo momento de segunda ordem obtidos para a quadratura amplitude dos feixes.

Os dados foram obtidos a partir de quatro medidas consecutivas de 450 mil pontos para cada potência de bombeio, variando a dessintonia das cavidades de análise. De cada um dos quatro conjuntos, foram selecionados 110 mil pontos correspondentes às flutuações de amplitude, totalizando 440 mil. Em seguida, foram calculados os momentos de ordem superior para grupos de 5 mil pontos, o que nos fornece 88 valores para cada momento. Finalmente, calculamos os valores médios e os respectivos desvios-padrão.

Na figura 5.16, apresentamos os resultados obtidos para os momentos e correlações de ordem superior, sendo a linha tracejada de cor vermelha a indicação do valor esperado para uma distribuição gaussiana. Vemos que os momentos de ordem par normalizados pelo momento de segunda ordem, indicados na figura 5.16(a) em escala log-linear, concordam com os valores calculados para uma distribuição normal. Em particular, a relação entre os momentos de terceira e segunda ordem (figura 5.16(b)) permite identificar se há alguma assimetria na distribuição dos dados, fato não observado nas flutuações medidas em nosso sistema, dado que o valor nulo encontrado para a obliquidade ("skewness") coincide com o valor esperado para uma distribuição gaussiana. Verificamos também a ausência de abaulamento ou achatamento através do cálculo da curtose (figura 5.16(c)). Essas duas grandezas são amplamente usadas na análise estatística como forma de identificar algum desvio do caráter gaussiano.

Outro cálculo pode ser realizado por meio da equação 4.6.8. Apresentamos na figura $5.16\left(\right.$ d) a razão $V_{i i j j} /\left(V_{i i} V_{j j}+2 V_{i j} V_{i j}\right)=1$. Mais uma vez observamos um excelente acordo entre as medidas e o resultado do cálculo para uma distribuição gaussiana. 


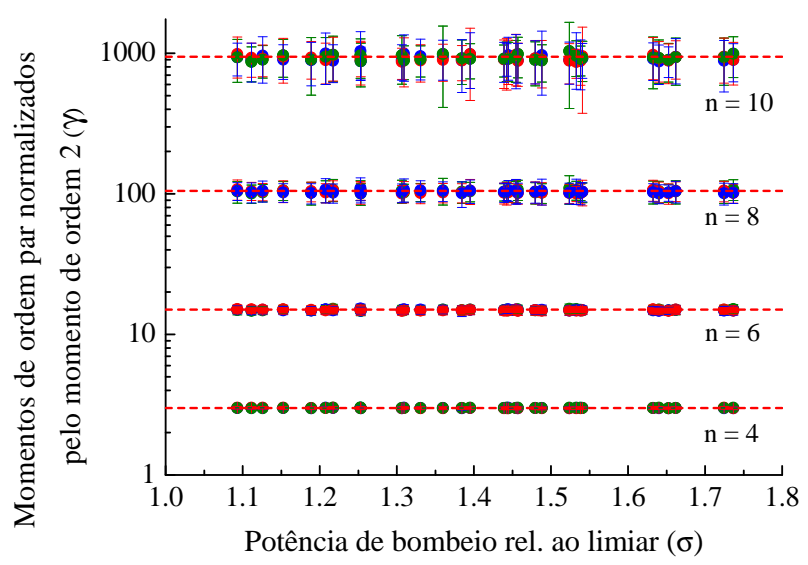

(a)

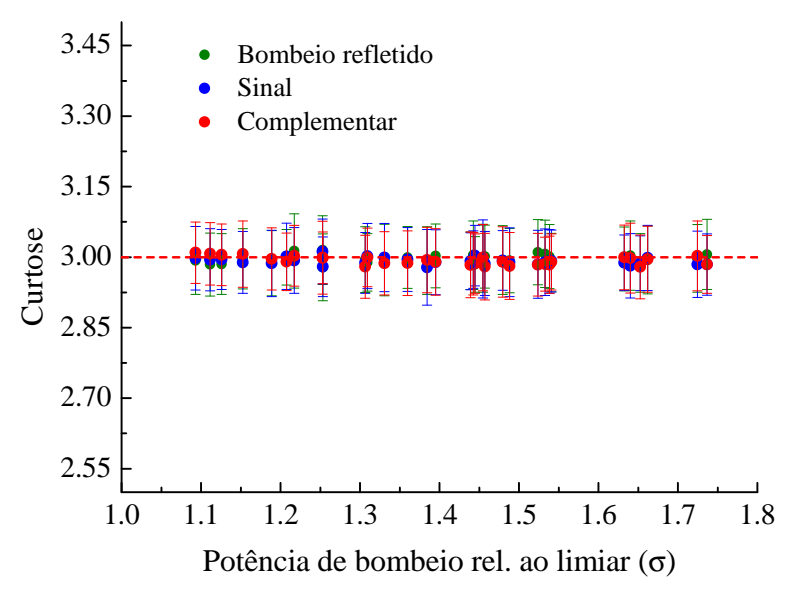

(c)

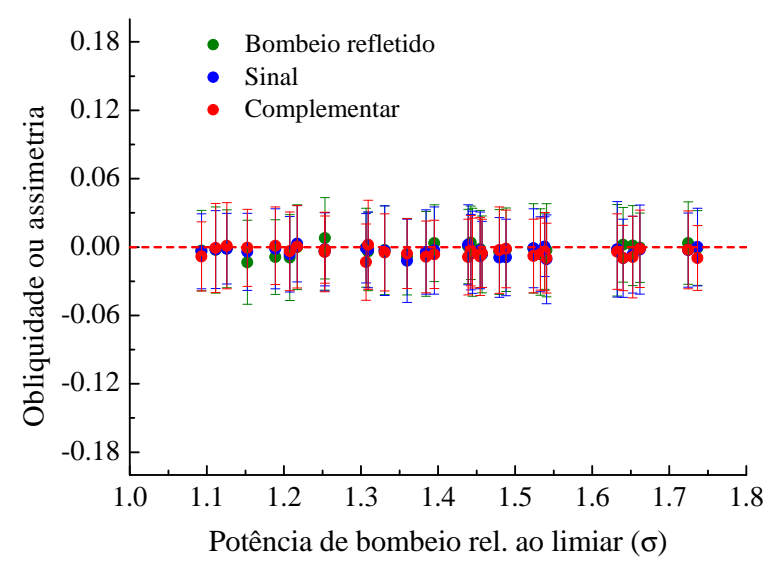

(b)

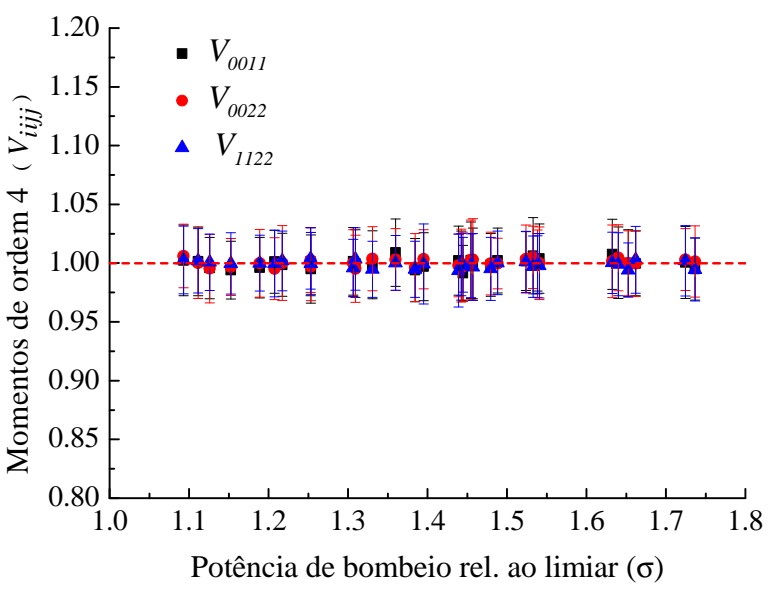

(d)

Figura 5.16: Momentos de ordem superior calculados a partir das flutuações de fase e amplitude de um dos feixes gêmeos. Em 5.16(a) nota-se o perfeito acordo com a relação $\gamma=(2 n-1)$ !!, em que $n$ especifica a ordem do momento. Nas figuras 5.16(b) e 5.16(c), vemos que todos os valores da curtose e da obliquidade ("skewness") são os mesmos obtidos por uma distribuição gaussiana. No gráfico 5.16(d) apresentamos correlações de ordem 4 do tipo $V_{i i j j}(i, j=0,1,2$ com $i \neq j)$. As linhas tracejadas em vermelho indicam os valores esperados para uma distribuição gaussiana.

Finalmente, mostramos os valores da curtose e obliquidade para flutuações obtidas durante toda a variação de dessintonia das cavidades de análise, a fim de mostrar que os valores são consistentes com uma distribuição normal, invariante no espaço de fases. A figura 5.17(a) mostra os ruídos de quadratura dos três campos normalizados pelo shotnoise como função da dessintonia da cavidade do feixe de bombeio. É clara a alternância entre as quadraturas amplitude e fase, conforme já discutido na figura 5.10. Nos gráficos 5.17 (b) e 5.17 (c), mostramos os valores da curtose e obliquidade obtidos para cada grupo de 5 mil pontos. Vemos um bom acordo entre os dados experimentais e a teoria, indicada 


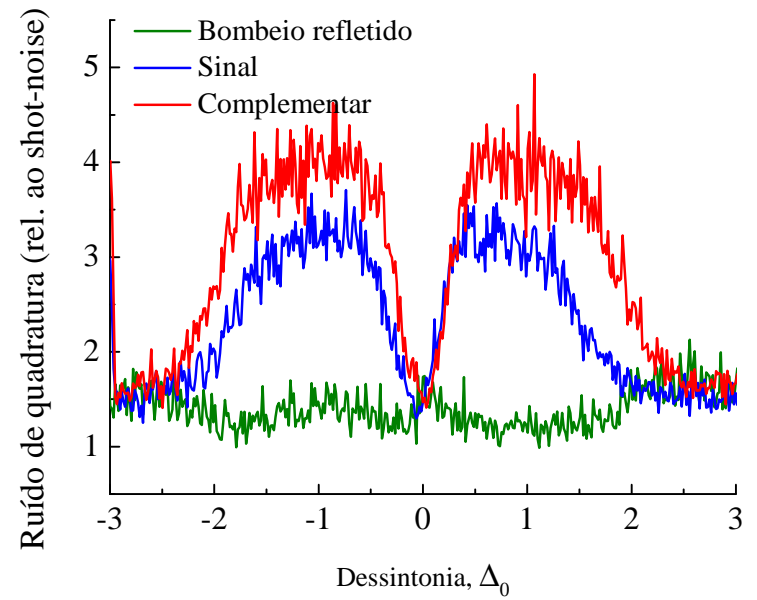

(a)

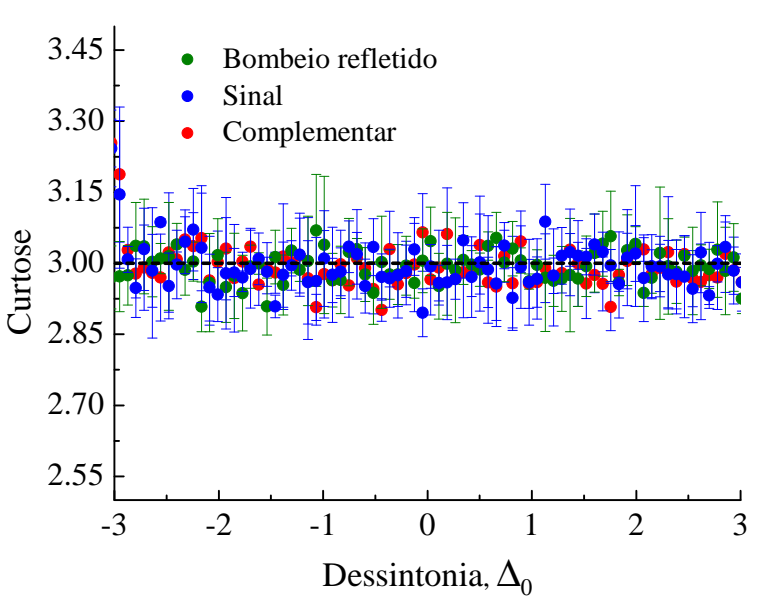

(b)

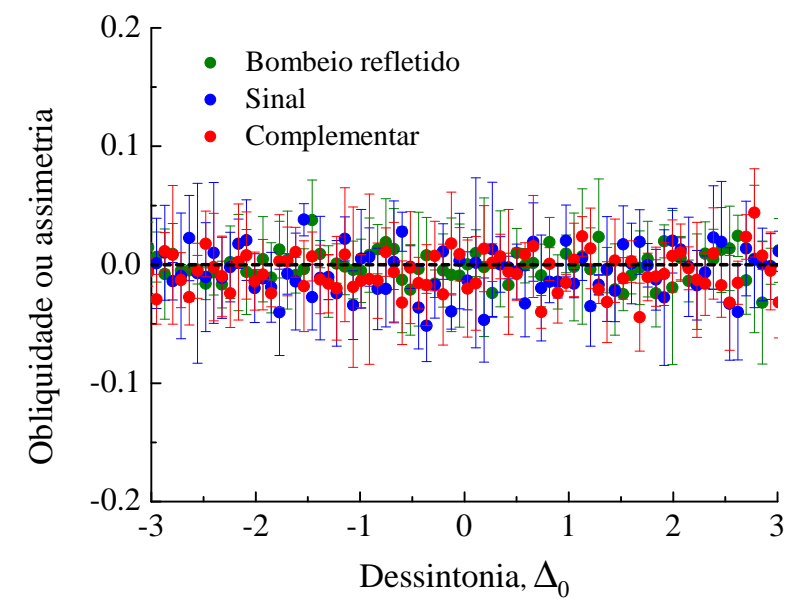

(c)

Figura 5.17: Correlações cruzadas de ordem 4 entre as flutuações dos campos bombeio refletido, sinal e complementar.

pelas linhas tracejadas de cor preta.

Com esta análise, concluímos que não há nenhuma evidência experimental de nãogaussianidade em nosso sistema. Portanto, sendo os estados dos campos gaussianos, a violão do critério PPT é uma condição necessária e suficiente para comprovar a existência de emaranhamento em nosso sistema, validando a verificação do análogo à morte súbita de emaranhamento para variáveis contínuas [Coelho 2009b, Barbosa 2010a].

Neste capítulo, apresentamos a primeira geração direta de emaranhamento entre três feixes intensos, produzidos por um OPO operando acima do limiar de oscilação. Tipificamos o emaranhamento tripartite e biparte quando submetemos os campos a perdas controladas e assinalamos a presença de uma estrutura similar à encontrada em estados 
GHZ, já demonstrado experimentalmente no âmbito das variáveis discretas. Dados os requisitos exigidos pelo critério de emaranhamento utilizado, caracterizamos a estatística das flutuações dos campos, comprovando com excelente precisão o carácter gaussiano do sistema. 


\section{Capítulo 6}

\section{Concluões e perspectivas}

Queremos saber,

O que vão fazer

com as novas invenções...

G. Gil (1976)

Apresentamos aqui a primeira medida de emaranhamento tripartite entre os feixes sinal, complementar e bombeio refletido produzidos pelo Oscilador Paramétrico Ótico (OPO) acima do limiar de oscilação, tendo como característica principal a diferença entre seus comprimentos de onda. Trata-se da primeira geração direta de emaranhamento de mais de dois subsistemas em variáveis contínuas [Coelho 2009b].

Tendo em vista a aplicação do OPO em processos envolvendo troca de informação quântica, investigamos a dinâmica do emaranhamento por ele produzido quando submetemos os campos a perdas. Foram estudados os casos tripartite e bipartite, resultando na primeira demonstração experimental do fenômeno análogo à morte súbita de emaranhamento em variáveis contínuas. Vimos que o controle do excesso de ruído gerado pela interação dos campos com fônons da rede cristalina acarreta uma transição do estado emaranhado de frágil para robusto e vice-versa. No primeiro caso o emaranhamento se perde para uma atenuação finita enquanto, no segundo, se mantém até que os campos sejam completamente perdidos [Coelho 2009b, Barbosa 2010a].

No que diz respeito à aplicação do critério de positividade sob transposição parcial (PPT), uma violação mais expressiva pode ser obtida utilizando elementos óticos de melhor qualidade na montagem do OPO. Além disso, uma melhoria no sistema de resfriamento do cristal possibilitaria atingir temperaturas ainda mais baixas, reduzindo ainda mais o efeito do ruído de fônons.

Consideramos ser de fundamental importância entender as nuances desse sistema dadas as perspectivas de aplicações futuras. A médio prazo temos o teletransporte entre 
cores distintas, que visa transferir o estado de um dos feixes gêmeos para o outro. Este é um projeto que está em seu estágio inicial.

A longo prazo desejamos criar uma rede de informação quântica, que consiste na interação entre diferentes sistemas físicos, usando o emaranhamento como recurso. Para tanto, está sendo construído um novo OPO com feixe de bombeio em $780 \mathrm{~nm}$, capaz de interagir com átomos de rubídio, também em estudo no LMCAL. Os feixes gêmeos gerados, emaranhados com o bombeio, terão comprimentos de onda em torno de 1560 nm, passíveis de serem enviados por fibra ótica com baixo ruído, com o propósito de gerar comunicação com outros sistemas, simulando uma rede quântica de informação.

Os resultados obtidos nesta tese dão continuidade a um trabalho que vêm sendo desenvolvido há aproximadamente 10 anos no LMCAL. O aparato experimental começou a ser montado em meados dos anos 2000, possibilitando as primeiras tentativas de medir o emaranhamento envolvendo os feixes gêmeos por volta de 2002 [Villar 2004], gerado com sucesso em meados de 2005. Juntamente com este resultado, novos fenômenos despertaram grande interesse no sistema, como, por exemplo, o emaranhamento tripartite demonstrado teoricamente em 2007 [Villar 2007b] e o excesso de ruído espúrio, que limitava a comprovação experimental do emaranhamento, mesmo sendo observadas correlações entre os três campos [Cassemiro 2008]. Um passo bastante importante foi a compreensão do excesso de ruído espúrio desde a sua caracterização [César 2008] até o seu controle, o que permitiu obter resultados preliminares de emaranhamento entres os três campos produzidos pelo OPO [Coelho 2009a], culminando nos resultados que apresentamos nesta tese. 


\section{Referências Bibliográficas}

[Adesso 2006] G. Adesso, Entanglement of Gaussian State, Tese de Doutorado, Università degli Studi diSalerno, 2006.

[Almeida 2007] M. P. Almeida, F. de Melo, M. Hor-Meyll, A. Salles, S. P. Walborn, P. H. Souto Ribeiro, L. Davidovich, Environment-Induced Sudden Death of Entanglement, Science 316, 579 (2007).

[Aolita 2008] L. Aolita, A física do emaranhamento: produção, detecção, e aplicações, Tese de Doutorado, Instituto de Física da Universidade Federal do Rio de Janeiro, 2008. Disponível em: [Disponível em: http://teses.ufrj.br/IF $/$ /MarioLeandroAolita.pdf]

[Armstrong 1962] J. A. Armstrong, N. Bloembergen, J. Ducuing and P. S. Pershan, Interactions between light waves in a nonlinear dielectric, Phy. Rev 127, 1918 (1962).

[Aspect 1981] A. Aspect, P. Grangier and G. Roger, Experimental tests of realistic local theories via Bell's theorem, Phys. Rev. Lett. 47, 460 (1981).

[Aspect 1982] A. Aspect, P. Grangier and G. Roger, Experimental Realization of EinsteinPodolsky-Rosen-Bohm Gedankenexperiment: A New Violation of Bell's Inequalities, Phys. Rev. Lett. 49, 91 (1982).

[Aspect 1982b] Alain Aspect, J. Dalibard and G. Roger, Experimental test of Bell's inequalities using time-varying analyses, Phys. Rev. Lett. 49, 1804 (1982).

[Asvany 2007] O. Asvany, E. Hugo, F. Müller, F. Kühnemann, S. Schiller,J. Tennyson and S. Schlemmer, Overtone spectroscopy of $\mathrm{H}_{2} \mathrm{D}^{+}$and $\mathrm{D}_{2} \mathrm{H}^{+}$using laser induced reactions J. Chem. Phys. 127, 154317 (2007).

[Auria 2010] V. D’Auria, S. Fornaro, A. Porzio, S. Solimeno, S. Olivares, and M. G. A. Paris, Characterization of bipartite Gaussian states from OPO, Phys. Scr. T140, 014018 (2010).

[Bachor 1998] H. A. Bachor, A Guide to Experiments in Quantum Optics (Wiley-VCH, 1998). 
[Barbosa 2010a] F. A. S. Barbosa, A. S. Coelho, A. J. de Faria, K. N. Cassemiro, A. S. Villar, P. Nussenzveig and M. Martinelli, Robustness of bipartite Gaussian entangled beams propagating in lossy channels, Nature Photonics 4, 858 (2010).

[Barbosa 2010b] F. A. S. Barbosa, A. J. de Faria, A. S. Coelho, K. N. Cassemiro, A. S. Villar, P. Nussenzveig, and M. Martinelli. Disentanglement in bipartite continuousvariable systems, Phys. Rev. A 84, 052330 (2011).

[Barbosa 2012] F. A. S. Barbosa, A. S. Coelho, K. N. Cassemiro, P. Nussenzveig, M. Martinelli, C. Fabre, and A. S. Villar, Quantum state reconstruction of spectral field modes: homodyne and resonator detection schemes. Em avaliação (2012).

[Bell 1964] J. S. Bell, On the Einstein-Podolsky-Rosen paradox, Physics 1, 195 (1964).

[Bell 1971] J. S. Bell, Proceedings of the International School of Physics 'Enrico Fermi', course IL: Foundations of Quantum Mechanics. Academic Press, New York, 171-181 (1971). Reproduzido em J. S. Bell, Speakable and unspeakable in quantum mechanics, Cambridge U. Press, Cambridge, 1987.

[Bennett 1992] C. H. Bennett and S. J. Weisner, Communication via one- and two-particle operators on Einstein-Podolsky-Rosen states, Phys. Rev. Lett 69, 2881 (1992)

[Bennett 1993] C. H. Bennett, G. Brassard, C. Crépeau, R. Jozsa, A. Peres and W. K. Wooters, Teleporting an Unknown Quantum State via Dual Classical and EinsteinPodolsky-Rosen Channels, Phys. Rev. Lett. 70, 1895 (1993).

[Black 2001] Eric D. Black, An introduction to Pound-Drever-Hall laser frequency stabilization, Am. J. Phys. 69, 79 (2001).

[Bohm 1951] D. Bohm, Quantum Theory (Prentice-Hall Inc., 1951).

[Bohm 1952a] D. Bohm, A suggested interpretation of the quantum theory in terms of hidden variables I, Phys. Rev. (2) 85, 166-179 (recebido em 5 julho 1951, publicado no volume $\mathrm{N}^{\circ} .2$ de 15 janeiro 1952).

[Bohm 1952b] D. Bohm, A suggested interpretation of the quantum theory in terms of hidden variables II, Phys. Rev. (2) 85, 180-193 (recebido em 5 julho 1951, publicado no volume $\mathrm{N}^{\circ} .2$ de 15 janeiro 1952).

[Bohr 1935] N. Bohr, Can Quantum-Mechanical Description of Physical Reality be Considered Complete?, Phys. Rev. 48, 696 (1935).

[Bosenberg 1996] W. R. Bosenberg, A. Drobshoff, J. L. Alexander, L. E. Myers and R. L. Byer, $93 \%$ pump depletion, 3.5 W continuous-wave, singly resonant optical parametric oscillator, Opt. Lett 21, 1336 (1996). 
[Braunstein 2005] S. L. Braunstein and Peter van Loock, Quantum information with continuos variable, Reviews of Modern Physics, 77, (2005).

[Breunig 2011 ] I. Breunig, D. Haertle and K. Buse, Continuous-wave optical parametric oscillators: recent developments and prospects, Appl Phys B 105:99-111 (2011).

[Burnham 1970] D. C. Burnham and D. L. Weinberg, Observation of Simultaneity in Parametric Production of Optical Photon Pairs, Phys. Rev. Lett. 25, 84 (1970).

[Byer 1968] R. L. Byer, M. K. Oshman, J. F. Young and S. E. Harris, Visible CW parametric oscillator, Appl. Phys. Lett 13, 109 (1968).

[Cassemiro 2007] K. N. Cassemiro, A. S. Villar, M. Martinelli and P. Nussenzveig, The quest for three-color entanglement: experimental investigation of new multipartite quantum correlations, Opt. Express 15, 18236 (2007).

[Cassemiro 2008] K. N. Cassemiro, Correlações Quânticas Multicolor no Oscilador Paramétrico Ótico, Tese de Doutorado, Universidade de São Paulo, 2008. [Disponível em: http://axpfep1.if.usp.br/ nadyne/]

[César 2008] J. E. S. César, Excesso de Ruído no Oscilador Paramétrico Ótico, Dissertação de Mestrado, Universidade de São Paulo, 2008.

[César 2008b] J. E. S. César, A. S. Coelho, K. N. Cassemiro, A. S. Villar, M. Lassen, P. Nussenzveig, and M. Martinelli, Extra phase noise from thermal uctuations in nonlinear optical crystals, Phys. Rev. A 79, 063816 (2009).

[Clauser 1978] J. F. Clauser and A. Shimony, Rep. Progr. Phys. 41, 1881 (1978).

[Coelho 2009a] A. S. Coelho, Emaranhamento Tripartite no Oscilador Paramétrico, Dissertação de Mestrado, Universidade de São Paulo, 2009. [Disponível em: http://www.teses.usp.br/teses/disponiveis/43/43134/tde-05062009-094713/]

[Coelho 2009b] A. S. Coelho, F. A. S. Barbosa, K. N. Cassemiro, A. S. Villar, M. Martinelli, and P. Nussenzveig, Three-Color Entanglement, Science 326, 823 (2009). Published Online September 17, 2009 (DOI:10.1126/science.1178683).

[Cohen-Tannoudji 1977] C. Cohen-Tannoudji, B. Diu and F. Laloë, Quantum Mechanics, Volume 1 (Wiley Interscience, 1977).

[Cohen-Tannoudji 2004] C. Cohen-Tannoudji, J. Dupont-Roc, G. Grynberg, Photons \& Atoms Introduction to Quantum Electrodynamics (Wiley-VCH, 2004).

[Collett 1987] M. J. Collett, R. Loudon and C. W. Gardiner, Quantum theory of optical homodyne and heterodyne detection, J. Mod. Opt 34, 881 (1987) 
[Cristescu 2008] S.M. Cristescu, S.T. Persijn, S. te Lintel Hekkert and F.J.M. Harren, Laser-based systems for trace gas detection in life sciences, Appl. Phys. B: Lasers Opt. 92, 343 (2008)

[Debuisschert 1993] T. Debuisschert, A. Sizmann, E. Giacobino and C. Fabre, Type-ii continuous-wave optical parametric oscillators: oscillation and frequency-tuning characteristics, J. Opt. Soc. Am., vol. B 10, pp. 1668-1680(1993).

[Dechoum 2010] K. Dechoum, M. D. Hahn, R. O. Vallejos and A. Z. Khoury, Semiclassical Wigner distribution for a two-mode entangled state generated by an optical parametric oscillator, Phys. Rev. A 81043834 (2010).

[Duan 2000] L.-M. Duan, G. Giedke, J. I. Cirac and P. Zoller, Inseparability criterion for continuous variable systems, Phys. Rev. Lett. 84, 2722 (2000); arXiv:quant$\mathrm{ph} / 9908056$.

[Dür 2000] W. Dür, G. Vidal, and J. I. Cirac, Three qubits can be entangled in two inequivalent ways, Phys. Rev. A 62, 062314 (2000)

[Einstein 1935] A. Einstein, B. Podolsky and N. Rosen, Can Quantum-Mechanical Description of Physical Reality Be Considered Complete?, Phys. Rev. 47, 777 (1935).

[Ekert 1991] A. K. Ekert, Quantum cryptography based on Bell's theorem, Phys. Rev. Lett.67, 661 (1991).

[Falk 1971] J. Falk, Instabilities in doubly resonant parametric oscillators: A theoretical analysis, IEEE J. Quantum Electron. 7, 230 (1971).

[Fox 2000] Mark Fox, Quantum Optics: An Introduction(Oxford Univ. Press, Oxford, 2005).

[Freedman 1972] S. J. Freedman, J. F. Clauser, Experimental Test of Local HiddenVariable Theories, Phys. Rev. Lett. 28, 938 (1972).

[Franken 1961] P. A. Franken, A. E. Hill, C. W. Peters, and G. Weinreich, Generation of Optical Harmonics, Phys. Rev. Lett. 7, 4 (1961).

[Franken 1963] P. A. Franken and J. F. Ward, Optical harmonics and nonlinear phenomena, Rev. Mod. Phys 35, 23 (1963).

[Furusawa 1998] A. Furusawa, J. L. Sørensen, S. L. Braunstein, C. A. Fuchs, H. J. Kimble and E. S. Polzik, Unconditional Quantum Teleportation, Science 282, 706 (1998).

[Galatola 1991] Galatola, L. A. Lugiato, M. G. Porreca, P. Tombesi and G. Leuchs, System control by variation of the squeezing phase, Opt. Commun. 85, 95 (1991). 
[Gardiner 1991] C. W. Gardiner, Quantum Noise (Springer Verlag, 1991).

[Giordmaine 1965] J. A. Giordmaine and R. C. Miller, Tunable coherent parametric oscillation in LiNbO3 at optical frequencies, Phys. Rev. Lett. 14, 973 (1965).

[Glauber 1963a] R.J. Glauber, Coherent and incoherent states of radiation field,Phys. Rev. 1312766 (1963).

[Glauber 1963b] R. J. Glauber, The Quantum Theory of Optical Coherence, Phys. Rev. 130, 2529 (1963).

[Greenberger 2009] Daniel Greenberger, Klaus Hentschel and Friedel Weinert (Editors), Compendium of Quantum Physics: Concepts, Experiments, History and Philosophy, Springer-Verlag (2009).

[Gröblacher 2007] Simon Gröblacher, Tomasz Paterek, Rainer Kaltenbaek, Caslav Brukner, Marek Żukowski, Markus Aspelmeyer and Anton Zeilinger, An experimental test of non-local realism, Nature 446 (2007).

[Grynberg 2010] Gilbert Grynberg, Alain Aspect and Claude Fabre, Introduction to Quantum Optics:From the Semi-classical Approach to Quantized Light, (2010).

[Heidmann 1987] A. Heidmann, R. J. Horowicz, S. Reynaud, E. Giacobino, C. Fabre and G. Camy, Observation of Quantum Noise Reduction on Twin Laser Beams, Phys. Rev. Lett. 59, 2555 (1987).

[Herpen 2006] M.M.J.W. Van Herpen, A.K.Y. Ngai, S.E. Bisson, J.H.P. Hackstein, E.J. Woltering and F.J.M. Harren, Optical parametric oscillator-based photoacoustic detection of $\mathrm{CO}_{2}$ at $4.23 \mu \mathrm{m}$ allows real-time monitoring of the respiration of small insects, Appl. Phys. B: Lasers Opt. 82, 665 (2006)

[Hofmann 2010] Helmut Hofmann, The Physics of Warm Nuclei, Oxford University Press.

[Holevo 2001] A. S. Holevo and R. F.Werner,Disentanglement in bipartite continuousvariable systems, Phys. Rev. A 63, 032312 (2001).

[Horodecki M. 1996] M. Horodecki, P. Horodecki and R. Horodecki, Separability of mixed states: necessary and sufficient conditions, Phys. Lett. A 223, 1 (1996).

[Horodecki R. 2007] R. Horodecki, P. Horodecki, M. Horodecki and K. Horodecki, Quantum entanglement, arXiv:quant-ph/0702225.

[Isaacson 2008] Walter Isaacson, Einstein: His Life and Universe, Editora Simon and Schuster, 2008. 
[Jagdish 2001] Jagdish Mehra and Helmut Rechenberg, The Historical Development of Quantum Mechanics, Vol. 6, Spring-Verlag New-York, 2001.

[Barata 2002] João C. A. Barata, Desigualdades de Bell, em 100 anos de Física Quântica, M. S. Hussein and S. R. A. Salinas, eds., Editora Livraria da Física (2002).

[Kasai 1997] K. Kasai, J. R. Gao and C. Fabre, Observation of squeezing using cascaded nonlinearity, Europhys. Lett. 40, 25 (1997).

[Kawakubo 2010] T. Kawakubo and K. Yamamoto, Optical homodyne detection in view of the joint probability distribution, Phys. Rev. A 82, 032102 (2010).

[Kimble 2008] H. J. Kimble, The quantum internet, Nature 453, 19 June (2008).

[Kocher 1967] C. A. Kocher and E. D. Commins, Polarization Correlation of Photons Emitted in an Atomic Cascade, Phys. Rev. Lett. 18, 575 (1967).

[Krauth 1998] Werner Krauth, Statistical Mechanics Algorithms and Computations (Oxford Master Series in Physics, 2006).

[Kroll 1962] N. M. Kroll, Parametric amplification in spatially extended media and application to the design of tunable oscillators at optical frequencies, Phys. Rev. 127, 1207 (1962).

[Laurat 2005] J. Laurat, L. Longchambon, C. Fabre and T. Coudreau, Experimental investigation of amplitude and phase quantum correlations in a type II optical parametric oscillator above threshold: from nondegenerate to degenerate operation, Opt. Lett. 30, 1177 (2005).

[Leonhardt 1997] Ulf Lenonhardt, Measuring the quantum states of light, Cambridge Studies on Modern Ligth, Cambridge University Press (2010).

[Leonhardt 2010] Ulf Lenonhardt, Essential quantum optics: from quantum measurements to black holes, Cambridge Studies on Modern Ligth, Cambridge University Press (1997).

[Levenson 1985] M. D. Levenson, R. M. Shelby, A. Aspect, M. Reid and D. F. Walls, Generation and detection of squeezed states of light by nondegenerate four-wave mixing in an optical fiber, Phys. Rev. A 32, 1550 (1985).

[Li 2002] X. Li, Q. Pan, J. Jing, J. Zhang, C. Xie and K. Peng, Quantum Dense Coding Exploiting a Bright Einstein-Podolsky-Rosen Beam, Phys. Rev. Lett. 88, 047904 (2002). 
[Maiman 1960] T. H. Maiman, Stimuled Optical Radiation in Ruby, Nature 187, 493 (1960).

[Mandel 1995] L. Mandel and E. Wolf, Optical Coherence and Quantum Optics(Cambridge University Press, 1995).

[Marek 2008] P. Marek, H. Jeong and M. S. Kim, Generating "squeezed"superpositions of coherent states using photon addition and subtraction, Phys. Rev. A 78, 063811 (2008).

[Martinelli 2002] M. Martinelli, Compressão de Ruído Quântico e Efeitos Transversos em Osciladores Paramétricos Óticos, Tese de Doutorado, Universidade de São Paulo, 2002. [Disponível em: http://axpfep1.if .usp.br/ lmcal]

[Marushkevich 2011] K. Marushkevich,M. Siltanen,M. Rasanen, L. Halonen and L. Khriachtchev, Identification of New Dimers of Formic Acid: The Use of a Continuous-Wave Optical Parametric Oscillator in Matrix Isolation Experiments, J. Phys. Chem. Lett. 2, 695 (2011).

[Nielsen 2000] M. A. Nielsen and I. L. Chuang, Quantum Computation and Quantum Information (Cambridge Univ. Press, Cambridge, 2000).

[Ourjoumtsev 2007] A. Ourjoumtsev, H. Jeong, R. Tualle-Brouri and P. Grangier, Generation of optical 'Schr\}odinger cats' from photon number states, Nature 448, 784 (2007).

[Parigi 2007] ,Valentina Parigi, Alessandro Zavatta, Myungshik Kim and Marco Bellini, Probing quantum commutation rules by addition and subtraction of single photons to/from a light field, Science 317, 5846 (2007).

[Paz 2008] J. P. Paz, A. J. Roncaglia, Dynamics of the Entanglement between Two Oscillators in the Same Environment, Phys. Rev. Lett. 100, 220401 (2008).

[Pearle 1970] P. M. Pearle, Hidden-Variable Example Based upon Data Rejection, Phys. Rev. D 2, 1418-1425 (1970).

[Peres 1978] Asher Peres, Unperformed experiments have no results, Am. J. Phys. 46, 745 (1978).

[Peres 1996] A. Peres, Separability Criterion for Density Matrices, Phys. Rev. Lett. 77, 1413 (1996).

[Ph77 Caltech] Ph 77 - The advanced physics lab at Caltech, Expt.72 - Laser Frequency Stabilization. Disponível em: http://www.its.caltech.edu/ ph76a/pdh.pdf 
[Piza 2009] A. F. R. de Toledo Piza, Sistemas quânticos compostos e emaranhamento, sistemas quânticos abertos e decoerência, notas de aula da Escola de Física Teórica de Departamento de Física Matemática , IFUSP (2009).

[Piza 2010] A. F. R. de Toledo Piza, Mecânica Quântica(EDUSP, 2010).

[Raymond 2008] Raymond Chiao and John Garrison, Quantum Optics, Oxford Graduate Texts 2008.

[Reid 1988] M. D. Reid and P. D. Drummond, Quantum correlations of phase in nondegenerate parametric oscillation, Phys. Rev. Lett. 60, 2731 (1988).

[Reid 1989] M. D. Reid and P. D. Drummond, Correlations in nondegenerate parametric oscillation-squeezing in the presence of phase diffusion, Phys. Rev. A 40, 4493(1989).

[Rigolin 2005] G. G. Rigolin, Estados Quânticos Emaranhados, Tese de Doutorado, Universidade Estadual de Campinas, 2005. [Disponível em: http://libdigi.unicamp.br/document/?code=vtls000360979]

[Roos 2004] Christian F. Roos, Mark Riebe, Hartmut H\}affner, Wolfgang H\}ansel, Jan Benhelm, Gavin P. T. Lancaster, Christoph Becher, Ferdinand Schmidt-Kaler and Rainer Blatt, Control and Measurement of Three-Qubit Entangled States, Science Vol. 304 (2004).

[Rosenfeld 1967] Niels Bohr in the thirties, in Niels Bohr. His Life and Work etc. (S. Rozental, ed., 1967), pp. 115-136.

[Salinas 1997] Silvio R. A. Salinas, Introdução à Física Estatística, São Paulo, Ed. Universidade de São Paulo, 1997.

[Schottky 1918] W. Schottky, Ann. Phys. (Leipzig) 57, 541 (1918).

[Schrödinger 1935] E. Schrödinger, Naturwissenschaften 23, 807 (1935).

[Schrödinger 1935, English translation by John D. Trimmer (1980)] E. Schrödinger, Proceedings of the American Society, 124, 323-38 (1980).

[Scully 1997] M. O. Scully and M. S. Zubairy, Quantum Optics (Cambridge University Press, 1997).

[Silberhorn 2002] Ch. Silberhorn, N. Korolkova and G. Leuchs, Quantum Key Distribution with Bright Entangled Beams, Phys. Rev. Lett. 88, 167902 (2002).

[Simon 2000] R. Simon, Peres-Horodecki separability criterion for continuous variable systems, Phys. Rev. Lett. 84, 2726 (2000); arXiv:quant-ph/9909044. 
[Simon 2003] Christoph Simon and William T.M. Irvine, Robust Long-Distance Entanglement and a Loophole-Free Bell Test with Ions and Photons, Phys. Rev. Lett, 91, 1 (2003).

[Smith 1968] R. G. Smith, J. E. Geusic, H. J. Levinstein, J. J. Rubin, S. Singh and L. G. Van Uitert, Continuous optical parametric oscillation in $\mathrm{Ba}_{2} \mathrm{NaNb}_{2} \mathrm{O}_{15}$, Appl. Phys. Lett 12, 308 (1968).

[Spiegel 1980] D. R. Spiegel and R. J. Helmer, Shot-noise measurements of the electron charge: An undergraduate experiment., Am. J. Phys. 63(6) (1995).

[Stanford WebPage] Stanford Encyclopedia of Philosophy, http://plato.stanford.edu/.

[Stephen 1987] Stephen Howard and Sanat K. Roy, Coherent states of a harmonic oscillator, Am. J. Phys. 551109 (1987).

[Teich 1989] M. C. Teich and B. E. A. Saleh, Squeezed states of light, Quantum Opt. 1, 153-191 (1989)

[Träger 2007] Frank Tr\}ager (Ed.), Springer Handbook of Lasers and Optics, 2007.

[van Loock 2003] P. van Loock and A. Furusawa, Detecting genuine multipartite continuous-variable entanglement, Phys. Rev. A 67, 052315 (2003).

[Villar 2004] A. S. Villar, Estudo de Emaranhamento no Oscilador Paramétrico Ótico Não-degenerado Acima do Limiar, Dissertação de Mestrado, Universidade de São Paulo, 2004. [Disponível em: http://axpfep1.if .usp.br/ lmcal]

[Villar 2005] A. S. Villar, L. S. Cruz, K. N. Cassemiro, M. Martinelli and P. Nussenzveig, Generation of Bright Two-Color Continuous Variable Entanglement, Phys. Rev. Lett. 95, 243603 (2005).

[Villar 2006] A. S. Villar, M. Martinelli, C. Fabre and P. Nussenzveig, Direct Production of Tripartite Pump-Signal-Idler Entanglement in the Above-Threshold Optical Parametric Oscillator, Phys. Rev. Lett. 97, 140504 (2006).

[Villar 2007a] A. S. Villar, K. N. Cassemiro, K. Dechoum, A. Z. Khoury, M. Martinelli and P. Nussenzveig, Entanglement in the above-threshold optical parametric oscillator, J. Opt. Soc. Am. B 24, 249 (2007).

[Villar 2007b] A. S. Villar, Emaranhamento Multicolor entre Feixes Intensos de Luz, Tese de Doutorado, Universidade de São Paulo, 2007. [Disponível em: http://axpfep1.if.usp.br/ villar/] 
[Villar 2008] A. S. Villar, The conversion of phase to amplitude fluctuations of a light beam by an optical cavity, Am. J. Phys. 76922 (2008).

[Vetterling 1980] William T. Veterling, Demonstrating Poisson Statistics, Am. J. Phys. 48(5) (1980).

[Walls 1995] D.F. Walls and G.J. Milburn, Quantum Optics (Springer Study Edition, 1995)

[Werner 2001] R. F. Werner and M.M. Wolf, Bound entangled Gaussian States, Phys. Rev. Lett. 86, 3658 (2001); arXiv:quant-ph/0009118.

[Wigner 1932] Eugene Wigner, On the Quantum Correction For Thermodynamic Equilibrium, Phys. Rev. A, 40749 (1932).

[Willke 1998] B. Willke, N. Uehara, E. K. Gustafson, R. L. Byer, P. J. King, S. U. Seel and R. L. Savage, Spatial and temporal filtering of a 10-W Nd:YAG laser with a Fabry-Perot ring-cavity premode cleaner, Opt. Lett. 23, 1704 (1998).

[Williamson 1936] J. Williamson, On the algebraic problem concerning the normal forms of linear dynamical systems, Am. J. Math. 58, 141 (1936).

[Wu 1986] L.A. Wu, H. J. Kimble, J. L. Hall and Huifa Wu, Generation of Squeezed States by Parametric Down Conversion, Phys. Rev. Lett. 57, 2520 (1986).

[Yang 1993] T. Yang, R. C. Eckardt and R. L. Byer, Continuouswave singly resonant optical parametric oscillator pumped by a single-frequency resonantly doubled Nd:YAG laser, Opt. Lett. 18, 971 (1993).

[Yariv 1989] A. Yariv, Quantum Eletronics, John Wiley and Sons (New York, Paris, 1989).

[Yu 2006] T. Yu, J. H. Eberly, Quantum Open System Theory: Bipartite Aspects, Phys. Rev. Letters 97, 140403 (2006).

[Yurke 1984] B.Yurke, Use of cavities in squeezed-state generation, Phys. Rev. A, 29, 408 (1984).

[Zavatta 2008] Alessandro Zavatta Valentina Parigi and Marco Bellini, Toward quantum frequency combs: Boosting the generation of highly nonclassical light states by cavity-enhanced parametric down-conversion at high repetition rates, Phys. Rev. A 78, 033809 (2008).

[Zeilinger 2007] Anton Zeilinger, Gregor Weihs, Thomas Jennewein and Markus Aspelmeyer, Happy centenary, photon, Nature 433, 20 de janeiro (2005). 
[Zhang 2001] K. S. Zhang, T. Coudreau, M. Martinelli, A. Maître and C. Fabre, Generation of bright squeezed light at $1.06 \mu \mathrm{m}$ using cascaded nonlinearities in a triply resonant cw periodically-poled lithium niobate optical parametric oscillator, Phys. Rev. A 64, 033815 (2001). 